MAGNO TEÓFILO MADEIRA DA SILVA

\title{
UM ESTUDO SOBRE TÉCNICAS DE EQUALIZAÇÃO AUTODIDATA
}

\author{
Tese apresentada à Escola Politécnica \\ da Universidade de São Paulo para \\ obtenção do Título de Doutor em \\ Engenharia Elétrica.
}


MAGNO TEÓFILO MADEIRA DA SILVA

\section{UM ESTUDO SOBRE TÉCNICAS DE EQUALIZAÇÃO AUTODIDATA}

Tese apresentada à Escola Politécnica da Universidade de São Paulo para obtenção do Título de Doutor em Engenharia Elétrica.

Área de Concentração:

Sistemas Eletrônicos

Orientadores:

Prof. Dr. Max Gerken

Profa. Dra. Maria D. Miranda 
Este exemplar foi revisado e alterado em relação à versão original, sob responsabilidade única do autor e com a anuência de seu orientador.

São Paulo, 27 de janeiro de 2005.

Magno Teófilo Madeira da Silva

Profa. Dra. Maria das Dores dos Santos Miranda

Silva, Magno Teófilo Madeira da

Um estudo sobre técnicas de equalização autodidata. São Paulo, 2005.

$194 \mathrm{p}$.

Edição Revisada

Tese (Doutorado) - Escola Politécnica da Universidade de São Paulo.

Departamento de Engenharia de Telecomunicações e Controle.

1. Equalização autodidata 2. Separação de fontes 3. Algoritmos adaptativos I. Universidade de São Paulo. Escola Politécnica. Departamento de Engenharia de Telecomunicações e Controle II.t 
Ao Professor Max Gerken

(in memorian) 


\section{Agradecimentos}

Meus agradecimentos ao Prof. Max Gerken, que me orientou com grande competência científica durante o mestrado e dois anos do doutorado. Obrigado Max, pela orientação cuidadosa, discussões técnicas valiosas, confiança, segurança e amizade.

À minha orientadora, Profa. Maria D. Miranda, pela orientação rigorosa, extrema dedicação e preocupação constante com o desenvolvimento do trabalho. Suas críticas e sugestões tiveram uma importância fundamental. Agradeço também pela paciência, confiança, amizade e preocupação com a minha carreira.

Ao Prof. Vítor H. Nascimento, pelas sugestões e discussões técnicas enriquecedoras.

Ao Prof. Luiz A. Baccalá, pelo apoio constante.

Ao Prof. J. R. C. Piqueira, pelo apoio e pelas sugestões dadas no exame de qualificação.

Ao Prof. Phillip M. S. Burt, pela leitura meticulosa da tese e pelas sugestões interessantes.

À toda minha família, em especial aos meus pais Maria e Maciel, pelo amor incondicional e permanente incentivo.

Ao amigo Eduardo F. Camacho, pelo companheirismo e pela convivência agradável.

A todos os colegas do Laboratório de Comunicações e Sinais, em especial ao amigo Pablo E. Jojoa, pelo apoio e pela convivência.

À Fundação de Amparo à Pesquisa do Estado de São Paulo (FAPESP), pelo financiamento deste trabalho. 


\section{Resumo}

Neste trabalho, investigam-se técnicas autodidatas baseadas em estatísticas de ordem superior, aplicadas à equalização de canais de comunicação. Inicialmente, obtém-se um intervalo do passo de adaptação que assegura a convergência do algoritmo do Módulo Constante com o gradiente exato. Algoritmos como o CMA (Constant Modulus Algorithm) e o SWA (Shalvi-Weinstein Algorithm) são revisitados e suas capacidades de tracking analisadas, utilizando-se uma relação de conservação de energia. Além disso, é proposto um algoritmo autodidata denominado AC-CMA (Accelerated Constant Modulus Algorithm) que utiliza a segunda derivada ("aceleração") da estimativa dos coeficientes. Esse algoritmo pode apresentar um compromisso mais favorável entre complexidade computacional e velocidade de convergência que o CMA e o SWA. Esses resultados são estendidos para o caso multiusuário. Através de simulações, os algoritmos são comparados e as análises de convergência e tracking validadas. Considerando o DFE (Decision Feedback Equalizer) no caso monousuário com o critério do módulo constante, é proposto um algoritmo concorrente que evita soluções degeneradas e apresenta um desempenho melhor do que os existentes na literatura. Com o intuito de evitar propagação de erros, é proposta uma estrutura híbrida que utiliza uma rede neural recorrente na malha de realimentação. Resultados de simulações indicam que seu uso pode ser vantajoso para canais lineares e não-lineares. 


\section{Abstract}

The equalization of communication channels is addressed by using blind techniques based on higher order statistics. A step-size interval is obtained to ensure the convergence of SteepestDescent Constant Modulus Algorithm. The Shalvi-Weinstein Algorithm (SWA) and Constant Modulus Algorithm (CMA) are revisited and their tracking capabilities are analyzed by using an energy conservation relation. Moreover, a novel blind algorithm named Accelerated Constant Modulus Algorithm (AC-CMA) is proposed. It adjusts the second derivative ("acceleration") of the coefficient estimates and presents a more favorable compromise between computational complexity and convergence rate than CMA or SWA. These results are extended to the MIMO (Multiple-Input Multiple-Output) case. By means of simulations, the algorithms are compared and the convergence and tracking analysis are validated. The Decision Feedback Equalizer (DFE) is considered in the SISO (Single-Input Single-Output) case with the Constant Modulus criterion and a concurrent algorithm is proposed. It avoids degenerated solutions and shows better behavior than the others presented in the literature. In order to avoid error propagation, a hybrid DFE is also proposed. It includes a recurrent neural network in the feedback filter and may be advantageously used to equalize linear and nonlinear channels. 


\section{Sumário}

Lista de figuras $\ldots \ldots \ldots \ldots \ldots \ldots \ldots \ldots \ldots$ vii

Lista de tabelas . . . . . . . . . . . . . . . . . . . . . . xii

Lista de abreviaturas . . . . . . . . . . . . . . . . xiv

Lista de símbolos . . . . . . . . . . . . . . . . . . . . . . xvii

1 Introdução e formulação do problema $\quad 1$

1.1 A desconvolução autodidata . . . . . . . . . . . . . . . . . . 1

1.2 A separação autodidata de fontes . . . . . . . . . . . . . . 5

1.3 Formulação do problema - caso SISO . . . . . . . . . . . . . 7

1.3.1 Condições para equalização perfeita . . . . . . . . . . . . . . . 11

1.3.2 O equalizador com realimentação de decisões . . . . . . . . . . . 16

1.3.3 Outros equalizadores não-lineares . . . . . . . . . . . . . . . . . 21

1.4 Formulação do problema - caso MIMO . . . . . . . . . . . . . . . . 23

1.5 Métodos para projeto de algoritmos adaptativos . . . . . . . . . . . 25

1.6 Objetivos e justificativa . . . . . . . . . . . . . . . . . . 27

1.7 Contribuições . . . . . . . . . . . . . . . . . . . . . . . . . . . . . . . . . . . . . 29

1.8 Organização da tese . . . . . . . . . . . . . . . . . . . . . . . 31

2 Algoritmos determinísticos para equalização monocanal 33

2.1 Cumulantes: definição e relações importantes . . . . . . . . . . . . . . . . 34

2.2 Funções custo associadas à equalização autodidata . . . . . . . . . . . . . . . 40

2.3 Gradientes e pontos estacionários . . . . . . . . . . . . . . . 44

2.4 Algoritmos do gradiente determinístico $\ldots \ldots \ldots \ldots$

2.5 O algoritmo Super-Exponencial no domínio da resposta combinada $\ldots . . .49$ 
2.6 Algoritmos no domínio dos coeficientes do equalizador . . . . . . . . . . 56

2.7 A convergência do SD-CMA . . . . . . . . . . . . . . . . . 60

2.8 Resultados experimentais . . . . . . . . . . . . . . . . . . . . 63

2.9 Conclusões . . . . . . . . . . . . . . . . . . . . . . . . . . 68

3 Algoritmos estocásticos para equalização monocanal $\quad 71$

3.1 Algoritmos decorrentes da estimativa instantânea do gradiente . . . . . . . . 71

3.2 O algoritmo do Módulo Constante Acelerado . . . . . . . . . . . . . . . . 75

3.2.1 O algoritmo "acelerador" de tempo contínuo . . . . . . . . . 76

3.2 .2 Métodos de discretização de Euler . . . . . . . . . . . . . . . . 77

3.2.3 O algoritmo "acelerador" para equalização autodidata . . . . . . . . . 78

3.3 Os algoritmos segundo o método quase-Newton . . . . . . . . . . . . 80

3.4 A relação de conservação de energia . . . . . . . . . . . . . . . . . . . . . 82

3.5 Análise de tracking . . . . . . . . . . . . . . . . . . 85

3.6 O problema da rotação de fase . . . . . . . . . . . . . . . . . . . . 89

3.7 Os algoritmos concorrentes . . . . . . . . . . . . . . . . . . . 89

3.8 Resultados experimentais . . . . . . . . . . . . . . . . . . . . . . . 90

3.8.1 Comparação com os algoritmos do gradiente determinístico . . . . . . 92

3.8 .2 Canais IIR . . . . . . . . . . . . . . . . . . . . . . . . . . 93

3.8.3 Canais FIR invariantes no tempo . . . . . . . . . . . . 95

3.8.4 Canal FIR variante no tempo . . . . . . . . . . . . . . . . . . . 97

3.8.5 Canal de rádio digital . . . . . . . . . . . . . . . . . 99

3.8.6 Algoritmos concorrentes . . . . . . . . . . . . . . . . . 101

3.8.7 Análise de tracking . . . . . . . . . . . . . . . . 104

3.9 Conclusões . . . . . . . . . . . . . . . . . . . . . . . 105

4 Algoritmos estocásticos para equalização multicanal 107

4.1 A função custo de Godard no caso MIMO . . . . . . . . . . . . . . . . . . 107

4.2 Algoritmos para equalização espaço-temporal . . . . . . . . . . . . . . . . 109

4.3 Análise de tracking . . . . . . . . . . . . . . . . . . . . . . . . . 114

4.4 Resultados experimentais . . . . . . . . . . . . . . . . 119 
4.5 Conclusões . . . . . . . . . . . . . . . . . . . . . . . . . . . . . . . 127

5 Sobre equalizadores com realimentação de decisões $\quad 129$

5.1 O comportamento ideal do filtro de realimentação . . . . . . . . . . . . . 129

5.2 O algoritmo que evita soluções degeneradas . . . . . . . . . . . . . . 131

5.3 O algoritmo concorrente para o DFE . . . . . . . . . . . . 134

5.4 A rede neural recorrente . . . . . . . . . . . . . . . . . . . . 137

5.5 O equalizador híbrido . . . . . . . . . . . . . . . . . 138

5.6 Resultados experimentais . . . . . . . . . . . . . . . . 141

5.6.1 Algoritmo concorrente para o DFE . . . . . . . . . . . . 141

5.6 .2 DFE híbrido . . . . . . . . . . . . . . . . . . . 145

5.7 Conclusões . . . . . . . . . . . . . . . . . . . . . . 148

6 Conclusões e trabalhos futuros $\quad 150$

$\begin{array}{ll}\text { Referências Bibliográficas } & 157\end{array}$

$\begin{array}{ll}\text { Apêndices } & 173\end{array}$

$\begin{array}{ll}\text { A Equalizadores sobre-amostrados } & 173\end{array}$

$\begin{array}{ll}\text { B Cálculos de gradientes } & 177\end{array}$

B.1 Derivada em relação à um vetor complexo . . . . . . . . . . . . . . . 177

B.2 Função custo de Godard . . . . . . . . . . . . . . . . . . . . . . . . . . . 179

B.3 Função custo de Shalvi-Weinstein . . . . . . . . . . . . . . . . . . . 179

B.4 Demonstração da Equação $(2.38)$. . . . . . . . . . . . . . . . . . . . . 180

C Sobre o algoritmo do Módulo Constante Acelerado 181

C.1 Dedução do AC-CMA . . . . . . . . . . . . . . . . . . . . . . 181

C.2 O AC-CMA segundo o método quase-Newton . . . . . . . . . . . . . 184

C.3 O AC-CMA e a conservação de energia . . . . . . . . . . . . . . . 185

$\begin{array}{ll}\text { D O algoritmo concorrente de Chen } & 188\end{array}$

E O algoritmo RTRL autodidata $\quad 192$ 


\section{Lista de Figuras}

1.1 Diagrama simplificado do problema de desconvolução autodidata. . . . . . . . . . 2

1.2 Relação entre os métodos HOS e os métodos minimização da entropia para desconvolução autodidata. . . . . . . . . . . . . . . . . . . 5 5

1.3 Diagrama simplificado do problema de separação de fontes. . . . . . . . . . . . . 6

1.4 Esquema de um sistema de comunicação com o equalizador linear transversal (LTE) implementado de forma autodidata. . . . . . . . . . . . . . . . . 8

1.5 Exemplo do efeito do canal; a) Sinal transmitido com 16-QAM, b) sinal que chega no receptor e c) sinal de saída do equalizador autodidata. . . . . . . . . . . . . 8

1.6 Modelo equivalente de um sistema de comunicação com equalizador sobre-amostrado

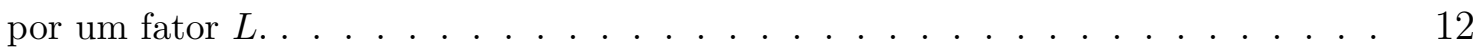

1.7 Módulo do operador de projeção $\mathcal{P}$ para o canal de rádio digital da referência [SPIB, 2001, chan4.mat]; $L=1, N_{c}=N_{h}=150$ e $M_{c}=100 \ldots \ldots \ldots \ldots$

1.8 Módulo do operador de projeção $\mathcal{P}$ para o canal de rádio digital da referência [SPIB, 2001, chan4.mat]; $L=2, N_{c}=N_{h} / 2=150$ e $M_{c}=150 \ldots \ldots$. . . . . . . . 16

1.9 Diagrama de blocos do DFE. . . . . . . . . . . . . . . . . 17

1.10 Modelo de um sistema de comunicação com DFE, assumindo-se decisões corretas.

1.11 Módulo do operador de projeção $\mathcal{P}$ para o canal $H(z)=1+z^{-1}+4 z^{-2}+2 z^{-3}+$ $4 z^{-4}+2 z^{-5}+z^{-6}+z^{-7}$, sem sobre-amostragem; a) LTE $(M=3)$; b) LTE $(M=10)$; c) $\operatorname{DFE}\left(M_{f}=3, M_{b}=5\right)$; d) $\operatorname{DFE}\left(M_{f}=3, M_{b}=7\right) \ldots \ldots \ldots$

1.12 Modelo de um sistema de equalização espaço-temporal, considerando $N_{u}=2$ usuários e $K=3$ antenas. . . . . . . . . . . . . . . . . . . .

2.1 Funções custo a) Godard, b) Shalvi-Weinstein e c) Godard reduzida; 2-PAM, canal H H $_{1} 43$ 
2.2 Comportamento do algoritmo no domínio da resposta combinada ao longo do tempo: a) inicialização, b) primeira iteração, c) segunda iteração. . . . . . . . . . . 50

2.3 ISI residual média (200 realizações) em função de $N_{b}$ do SEA, SD-SWA $\left(\beta_{S W}=0,1\right)$ e SD-CMA $\left(\mu=10^{-4}\right) ; 6$-PAM, canal $\mathrm{H}_{2}$ e $M=16 \ldots \ldots$. . . . . . . 64

2.4 ISI residual média (200 realizações) do SD-CMA para diferentes valores de $N_{b}$; $\mu_{n}=0,1 \mu_{n \max }, 6-\mathrm{PAM}$, canal $\mathrm{H}_{2}$ e $M=16 \ldots \ldots \ldots$

2.5 Passo de adaptação médio (200 realizações) do SD-CMA para diferentes valores de $N_{b} ; \mu_{n}=0,1 \mu_{n \max }, 6-\mathrm{PAM}$, canal $\mathrm{H}_{2}$ e $M=16 \ldots \ldots \ldots$

2.6 Média (200 realizações) dos coeficientes do SD-CMA para diferentes valores de $N_{b}$; $\mu_{n}=0,1 \mu_{n \max }, 6-\mathrm{PAM}$, canal $\mathrm{H}_{2}$ e $M=16 \ldots \ldots \ldots$

2.7 ISI residual média (200 realizações) do SD-CMA em função de $\mu_{n} ; N_{b}=600$, 6-PAM, canal $\mathrm{H}_{2}$ e $M=16$.

2.8 Média (200 realizações) dos coeficientes e do passo de adaptação do SD-CMA em função de $\mu_{n} ; N_{b}=600,6$-PAM, canal $\mathrm{H}_{2}$ e $M=16$.

3.1 Relações entre algoritmos autodidatas clássicos.

3.2 Relações entre os algoritmos de tempo contínuo e algoritmos autodidatas, $\mathbf{A}(t)=2 \mathbf{M}_{1}\left(\mathbf{M}_{2}+\mathbf{u}^{*}(t) \mathbf{u}^{T}(t) \mathbf{M}_{1} \mathbf{M}_{3}\right) \ldots \ldots \ldots \ldots$

3.3 Mapeamento sem perdas com realimentação para algoritmos da forma (3.24). . . . 83

3.4 Mapeamento sem perdas com realimentação de ganho para o AC-CMA. . . . . . 85

3.5 Relações entre os algoritmos concorrentes para adaptação autodidata do LTE. . . 91

3.6 ISI residual média (50 realizações); canal $\mathrm{H}_{2}, \mathrm{SNR}=30 \mathrm{~dB}, M=16$ e 6 PAM; SD-CMA $\left(\mu=10^{-4}, N_{b}=780\right)$; $\operatorname{SD-SWA~}\left(\beta_{S W}=0,1, N_{b}=780\right)$; $\operatorname{CMA}\left(\mu=1,46 \times 10^{-5}\right)$; e SWA $(\lambda=0,9975, \delta=0,0025) \ldots \ldots$

3.7 Curvas de nível da função custo de Godard para 2-PAM e canal $\mathrm{H}_{1}$ e trajetórias do SWA $(\lambda=0,995, \delta=0,005)$, CMA $(\mu=0,0016)$ e AC-CMA $\left(\mu=0,16, m_{1}=\right.$ $0,15, \kappa=100)$ inicializados nos pontos indicados; MG indica mínimo global e ML mínimo local.

3.8 ISI residual do SWA $(\lambda=0,995, \delta=0,005)$, CMA $(\mu=0,0016)$ e AC-CMA $\left(\mu=0,16, m_{1}=0,15, \kappa=100\right)$, inicializados em $\mathbf{w}(0)=\left[\begin{array}{ll}0,2 & -0,4\end{array}\right]^{T} ;$ canal $\mathrm{H}_{1}$,

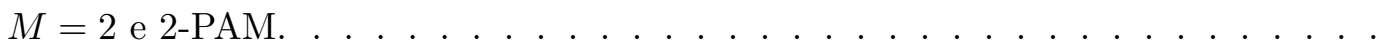


3.9 Curvas de nível da função custo de Godard para 2=PAM e canal $\mathrm{H}_{2}$ e trajetórias do SWA $(\lambda=0,995, \delta=0,005)$, CMA $(\mu=0,0024)$ e AC-CMA $\left(\mu=0,24, m_{1}=\right.$ $0,15, \kappa=100)$, inicializados nos pontos indicados; MG indica mínimo global e ML mínimo local. . . . . . . . . . . . . . . . . . .

3.10 ISI residual média ao longo das iterações do SWA $(\lambda=0,9975, \delta=0,0025)$, CMA $\left(\mu=6,2 \times 10^{-4}\right)$ e AC-CMA $\left(\mu=0,062, m_{1}=0,15, \kappa=100\right)$ para 4-QAM, $M=27$, média de 50 realizações e canais a) $\mathrm{H}_{4}$, b) $\mathrm{H}_{5}$, c) $\mathrm{H}_{6}$ e d) $\mathrm{H}_{7} \ldots \ldots$. . . . . . 96

3.11 Coeficientes do canal $\mathrm{H}_{8}$ ao longo das iterações. . . . . . . . . . . . . . . . . . .

3.12 a), b) e c) Erros na recuperação do sinal utilizando respectivamente o CMA ( $\mu=$ $0,0025)$, o SWA $(\lambda=0,995, \delta=0,005)$ e o AC-CMA $\left(\mu=0,25, m_{1}=0,15\right.$, $\kappa=100)$; d)Módulos dos zeros do canal $\mathrm{H}_{8}$ ao longo das iterações; e) ISI residual; $\mathrm{SNR}=20 \mathrm{~dB}, M=11$ e 2 -PAM $\ldots \ldots \ldots \ldots$

3.13 a) e b) Resposta em freqüência, c) Parte Real e d) Parte Imaginária dos coeficientes do canal $\mathrm{H}_{9}[$ SPIB, 2001, chan9.mat]. . . . . . . . . . . . . . . . . . .

3.14 ISI residual média (50 realizações); canal $\mathrm{H}_{9}$, $\mathrm{SNR}=25 \mathrm{~dB}, 4$-QAM, $M=6, L=2$; SWA $(\lambda=0,999, \delta=0,1)$; CMA $\left(\mu=2,5 \times 10^{-4}\right)$; e AC-CMA $\left(\mu=0,1, m_{1}=0,15\right.$, $\kappa=100) \ldots \ldots \ldots \ldots \ldots \ldots$

3.15 Sinal de saída do equalizador entre as iterações 14000 e 15000; $M=6, L=2$, canal $\mathrm{H}_{9}, \mathrm{SNR}=25 \mathrm{~dB}$ e 4-QAM; a) SWA $(\lambda=0,999, \delta=0,1)$; b) $\mathrm{CMA}(\mu=$ $\left.2,5 \times 10^{-4}\right) ;$ c) $\mathrm{AC}-\mathrm{CMA}\left(\mu=0,1, m_{1}=0,15, \kappa=100\right)$.

3.16 Resposta em freqüência do canal $\mathrm{H}_{10} \ldots \ldots \ldots$. . . . . . . . . . . . 101

3.17 ISI residual média (50 realizações); $M=23$, canal $\mathrm{H}_{10}, \mathrm{SNR}=30 \mathrm{~dB}$ e 16-QAM; $\lambda=0,9997 ; \delta=3 \times 10^{-4} ; \mu_{\mathrm{CMA}}=1,2 \times 10^{-4} ; \mu_{\mathrm{AC}}=5 \times 10^{-3} ; m_{1}=0,15 ; \kappa=100 ;$ $\mu_{d}=10^{-3} ; \rho=0,6$.

3.18 Sinal de saída do equalizador entre as iterações 19000 e 20000 dos algoritmos para $M=23$, canal $\mathrm{H}_{10}, \mathrm{SNR}=30 \mathrm{~dB}$ e 16 -QAM.

3.19 Curvas com resultados analíticos e de simulação do MSE; canal $\mathrm{H}_{11}, \mathbf{Q}=10^{-6} \mathbf{I}$, 6-PAM, $M=4, L=2$ e média de 100 realizações; a) CMA; b) SNLA; e c) SWA. . 104 
4.2 IR média (100 realizações) para o MU-SWA $\left(\lambda=0,9992, \delta=8 \times 10^{-4}\right)$, MU-CMA $\left(\mu=5 \times 10^{-5}, \varrho=3\right)$ e MU-AC-CMA $\left(\mu=0,05, m_{1}=0,15, \kappa=100, \varrho=3\right) ;$ $N_{u}=2, K=3, M_{u}=15$, inicialização: $(5 ; 7)_{15}$, 4-QAM, canal $\mathrm{H}_{12}$ e $\mathrm{SNR}=30 \mathrm{~dB} .121$

4.3 Sinais de saída dos equalizadores (Eq. 1 e Eq. 2) entre as iterações 59000 e 60000 para os algoritmos configurados como na simulação da Figura 4.2.

4.4 IR do a) MU-AC-CMA $\left(\mu=k \times 10^{-5}, \varrho=3\right)$ e MU-CMA $\left(\mu=k \times 10^{-5}, \kappa=100\right.$, $\left.m_{1}=0,15, \varrho=3\right)$, canal $\mathrm{H}_{12}, \mathrm{SNR}=30 \mathrm{~dB}, 4-\mathrm{QAM}, N_{u}=2, K=3, M_{u}=15$, inicialização: $(5 ; 7)_{15}$, Equalizador 1. . . . . . . . . . . . . . . . . . .

4.5 IR do a) MU-AC-CMA $\left(\mu=k \times 10^{-5}, \varrho=3\right)$ e MU-CMA $\left(\mu=k \times 10^{-5}, \kappa=100\right.$, $\left.m_{1}=0,15, \varrho=3\right)$, canal $\mathrm{H}_{12}, \mathrm{SNR}=30 \mathrm{~dB}, 4$-QAM, $N_{u}=2, K=3, M_{u}=15$, inicialização: $(8 ; 14)_{15}$, Equalizador 1.

4.6 a), b) e c) Erros na recuperação do sinal $a_{1}(n)$ para o MU-CMA $(\mu=0,01, \varrho=4)$, o MU-SWA $(\lambda=0,995, \delta=0,005)$ e o MU-AC-CMA $\left(\mu=0,99, m_{1}=0,15\right.$, $\kappa=100, \varrho=4)$ respectivamente; d) Interferência residual; SNR $=25 \mathrm{~dB}, 2-\mathrm{PAM}$, canal $\mathrm{H}_{13}, M_{u}=15, K=3$ e inicialização: $(5 ; 7)_{15}$.

4.7 a), b) e c) Erros na recuperação do sinal $a_{2}(n)$ para o MU-CMA $(\mu=0,01, \varrho=4)$, o MU-SWA $(\lambda=0,995, \delta=0,005)$ e o MU-AC-CMA $\left(\mu=0,99, m_{1}=0,15\right.$, $\kappa=100, \varrho=4)$ respectivamente; d) Interferência residual; $\mathrm{SNR}=25 \mathrm{~dB}, 2-\mathrm{PAM}$, canal $\mathrm{H}_{13}, M_{u}=15, K=3$ e inicialização: $(5 ; 7)_{15}$.

4.8 Curvas com resultados analíticos e de simulação do MSE; canal $\mathrm{H}_{14}, \mathbf{Q}=10^{-6} \mathbf{I}$, 4-PAM, $N_{u}=2, K=3, M_{u}=9$, inicialização: $(2 ; 5)_{9}$, média de 100 realizações, Equalizador 1; a) MU-CMA; e b) MU-SWA

5.1 Relações entre os algoritmos para adaptação autodidata do DFE. . . . . . . . . . 135

5.2 RNN com $N=3$ neurônios, $M$ entradas e saída $y_{3} \ldots \ldots \ldots \ldots$

5.3 Esquema do equalizador híbrido RNN-LC . . . . . . . . . . . . . . . . . . 139

5.4 Propagação de erro para adaptação da RNN. . . . . . . . . . . . . . . . . . . . 140

5.5 a) Resposta ao pulso unitário, b) e c) Resposta em freqüência do canal $\mathrm{H}_{15}$. . . . 142

5.6 Curvas de taxa de erro de símbolo (SER) em função da relação sinal ruído (SNR) para 16-QAM, $M_{f}=11, M_{b}=17$, canal $\mathrm{H}_{15}, \sigma_{d}=10, \mu=10^{-3}, \mu_{d}=10^{-2}$, $\mu_{\theta}=10^{-2}, \tilde{\rho}=0,06$. 
5.7 MSE baseado na decisão, média de 50 realizações, 16-QAM, canal $\mathrm{H}_{15}, \mathrm{SNR}=$ $22,5 \mathrm{~dB}, M_{f}=11$ e $M_{b}=17 ;$ LMS-DFE $(\mathrm{SPE})\left(\mu=2,5 \times 10^{-3}, \tau_{d}=10\right)$, DFECMA-FB $\left(\mu=2,5 \times 10^{-4}\right)$ e NDEG-SDD-CMA $\left(\mu=10^{-3}, \mu_{d}=10^{-2}, \tilde{\rho}=0,06\right)$.

5.8 Erros na recuperação do sinal para a) DFE-LMS (SPE) $\left(\mu=10^{-2}, \sigma_{d}=5\right)$, b) NDEG-SDD-CMA $\left(\mu=2 \times 10^{-3}, \mu_{d}=2 \times 10^{-2}, \tilde{\rho}=0,06\right)$, c) DFE-CMA-FB $\left(\mu=5 \times 10^{-4}, \tilde{\rho}=0,06, \mu_{\theta}=5 \times 10^{-3}\right)$; d) Módulos dos zeros do canal $\mathrm{H}_{16}$ ao longo das iterações; $M_{f}=11, M_{b}=17, \mathrm{SNR}=20 \mathrm{~dB}, 16$-QAM.

5.9 Curvas de taxa de erro de símbolo (SER) em função da relação sinal ruído (SNR) para $M_{f}=11, M_{b}=17$, canal $\mathrm{H}_{16}$ e 16-QAM. . . . . . . . . . . . . . 145

5.10 MSE baseado na decisão, média de 50 realizações, 16-QAM, canal $\mathrm{H}_{17}$, $\mathrm{SNR}=$ $25 \mathrm{~dB}$, DFE-CMA-FB $\left(M_{f}=5, M_{b}=4, \mu=10^{-4}\right)$, RNN-RTRL $(M=5, N=3$, $\left.\mu_{\mathrm{RTRL}}=10^{-3}\right)$, Híbrido $\left(M_{f}=5, M_{b}=M_{r}=4, N=3, \mu=10^{-4}, \mu_{\mathrm{RTRL}}=10^{-3}\right)$, $\mu_{\theta}=10^{-3} \ldots \ldots \ldots \ldots \ldots \ldots \ldots \ldots \ldots$

5.11 Curvas de taxa de erro de símbolo (SER) em função da relação sinal ruído (SNR) para LTE $(M=5), \operatorname{RNN}(M=3, N=3)$, DFE $\left(M_{f}=3, M_{b}=2\right)$, Híbrido $\left(M_{f}=3, M_{b}=M_{r}=2, N=3\right), 4$-QAM e canal $\mathrm{H}_{18}$.

5.12 a), b) e c) Erros na recuperação do sinal para a) LTE-CMA $\left(M=9, \mu=10^{-3}\right)$, b) DFE-CMA-FB $\left(M_{f}=5, M_{b}=4, \mu=10^{-3}\right)$, c) Híbrido $\left(M_{f}=5, M_{b}=M_{r}=4\right.$, $\left.\left.N=3, \mu=10^{-3}, \mu_{\mathrm{RTRL}}=10^{-2}\right), \mu_{\theta}=10^{-2} ; \mathrm{d}\right)$ Módulos dos zeros do canal $\mathrm{H}_{8}$ ao longo das iterações; SNR $=25 \mathrm{~dB}, 4$-QAM . . . . . . . . . . . . . . . . . 148

A.1 Diagrama de blocos de um sistema de comunicação com equalizador sobre-amostrado por um fator $L \ldots \ldots \ldots$. . . . . . . . . . . . . . . . . . . . . . . . . . 173

A.2 Modelo do canal considerando a decomposição polifásica por um fator $L$. . . . . . 175

A.3 Modelo do equalizador resultante da decomposição polifásica por um fator $L$. . . . 176

D.1 Ilustração das regiões locais para adaptação SDD e 64-QAM. . . . . . . . . . . . 190 


\section{Lista de Tabelas}

2.1 Gradientes das funções custo consideradas; $\bar{\beta}=\beta-1 \ldots$. . . . . . . . . . . . . . 44

2.2 Resumo dos algoritmos e análises de convergência do Capítulo $2 . \quad \ldots . . .69$

3.1 Sumário do algoritmo do Módulo Constante (CMA) . . . . . . . . . . . . . . 73

3.2 Sumário do Algoritmo de Shalvi-Weinstein (SWA) . . . . . . . . . . . . . 73

3.3 Sumário do Algoritmo do Módulo Constante Acelerado (AC-CMA). . . . . . . . . 80

3.4 Interpretação dos algoritmos aproximados segundo o método quase-Newton. . . . 82

3.5 MSE $(\zeta)$ em regime, parâmetros de adaptação ótimos $\left(\nu_{\mathrm{o}}\right)$ e valores mínimos do MSE em regime $\left(\zeta_{o}\right)$ para o CMA, SNLA e SWA. . . . . . . . . . . 88

3.6 Modelos dos canais usados nas simulações da Seção 3.8.3 . . . . . . . . . . . . . . 95

3.7 Resposta ao pulso unitário do canal $\mathrm{H}_{10} \ldots \ldots \ldots$. . . . . . . . . . . . 102

4.1 Sumário do MU-CMA. . . . . . . . . . . . . . . . . . . . . . . . . . . . 110

4.2 Sumário do MU-SWA . . . . . . . . . . . . . . . . . . . . . . 111

4.3 Sumário do MU-AC-CMA. . . . . . . . . . . . . . . . . . . . . . . . . 113

4.4 Complexidade computacional dos algoritmos autodidatas no caso MIMO para sinais reais, $\quad D=\left(N_{u}-1\right)\left(2 k_{1}+1\right), \quad N_{u} \geq 2 \ldots \ldots \ldots \ldots 114$

4.5 MSE $\left(\zeta_{i}\right)$ em regime, parâmetros de adaptação ótimos $\left(\nu_{\mathrm{o}}\right)$ e valores mínimos do MSE em regime $\left(\zeta_{o}\right)$ para o MU-CMA e o MU-SWA; $\mathbf{R}$ é a matriz de autocorrelação.118

4.6 Coeficientes dos canais $\mathrm{H}_{12}$ e $\mathrm{H}_{14} \ldots \ldots \ldots \ldots$. . . . . . . . . . . . 120

4.7 Número de erros na recuperação dos sinais $a_{1}(n)$ e $a_{2}(n)$ considerando $4 \times 10^{4}$ iterações dos algoritmos MU-SWA, MU-SWA e MU-AC-CMA. . . . . . . . . . . 124

5.1 Sumário do DFE-CMA-FB. . . . . . . . . . . . . . . . . . . . 134

5.2 Sumário do NDEG-SDD-CMA. . . . . . . . . . . . . . . . 136 
5.3 Complexidade computacional em termos de operações complexas por iteração. 136

5.4 Modelos dos canais utilizados nas simulações com o equalizador híbrido. . . . . . 146

6.1 Principais contribuições da tese. . . . . . . . . . . . . . . . . . . 152

C.1 Sumário do AC-CMA matricial. . . . . . . . . . . . . . . . . . . 183

D.1 Sumário do CMA+SDD . . . . . . . . . . . . . . . . . . . . . . . . . . . . 191 


\section{Lista de Abreviaturas}

A seguir são listadas as principais abreviações usadas na tese. No caso de siglas consagradas na literatura internacional, optou-se por manter as mesmas em inglês.

AAFA

AC-CMA

AWGN

BSS

CCI

CMA

$\mathrm{DD}$

DFE

DTV

FB-LC

f.d.p.

FF-LC

FIR

GP

HOS

ICA

i.i.d.
Accelerating Adaptive Filtering Algorithm (Algoritmo Acelerador para filtragem adaptativa)

Accelerated Constant Modulus Algorithm (Algoritmo do Módulo Constante Acelerado)

Additive White Gaussian Noise (Ruído gaussiano branco e aditivo)

Blind Source Separation (Separação autodidata de fontes)

Co-Channel Interference (Interferência co-canal)

Constant Modulus Algorithm (Algoritmo do Módulo Constante)

Decision-Direct (Decisão Direta)

Decision Feedback Equalizer (Equalizador com realimentação de de decisões)

Digital Television (TV digital)

Feedback Linear Combiner (Combinador linear de realimentação)

função densidade de probabilidade

Feedforward Linear Combiner (Combinador linear direto)

Finite Impulse Response (Resposta ao pulso unitário finita)

Gradiente Projetado

Higher Order Statistics (Estatísticas de ordem superior)

Independent Component Analysis

independente e identicamente distribuída 
IIR

IR

ISI

LC

LMS

LTE

MIMO

MISO

ML

MLP

MSE

MU-AC-CMA

MU-CMA

MU-SWA

NLMS

PAM

PTA

QAM

$\mathrm{RBF}$

RLS

RNN

RTRL

SD-CMA
Infinite Impulse Response (Resposta ao pulso unitário infinita)

Interferência Residual

Intersymbol Interference (Interferência intersimbólica)

Linear Combiner (Combinador linear)

Least-Mean-Square

Linear Transversal Equalizer (Equalizador linear transversal)

Multiple-Input Multiple-Output (Múltiplas entradas e múltiplas saídas)

Multiple-Input Single-Output (Múltiplas entradas e uma saída)

Maximum Likelihood (Máxima verossimilhança)

Multilayer Perceptron

Mean-Square-Error (Erro quadrático médio)

Multiuser Accelerated Constant Modulus Algorithm (Algoritmo do

Módulo Constante Acelerado Multiusuário)

Multiuser Constant Modulus Algorithm (Algoritmo do Módulo

Constante Multiusuário)

Multiuser Shalvi Weinstein Algorithm (Algoritmo de

Shalvi-Weinstein Multiusuário)

Non-Degenerative Soft Decision-Directed Constant Modulus Algorithm

(Algoritmo do Módulo Constante com Decisão Direta Suave

Não-Degenerativo)

Normalized Least-Mean-Square

Pulse Amplitude Modulation

Phase Tracking Algorithm (Algoritmo de rastreamento de fase)

Quadrature Amplitude Modulation

Radial Basis Function (Função de base radial)

Recursive Least-Squares

Recurrent Neural Network (Rede neural recorrente)

Real-Time Recurrent Learning (Aprendizagem recorrente em tempo real)

Steepest-Descent Constant Modulus Algorithm (Algoritmo do 
Módulo Constante do gradiente determinístico)

SDD

Soft Decision-Direct (Decisão Direta Suave)

SD-SWA

Steepest-Descent Shalvi-Weinstein Algorithm (Algoritmo de

Shalvi-Weinstein do gradiente determinístico)

SEA

Super-Exponential Algorithm (Algoritmo Super-Exponencial)

SER

Symbol Error Rate (Taxa de erro de símbolo)

SIMO

Single-Input Multiple-Output (Uma entrada e múltiplas saídas)

SISO

Single-Input Single-Output (Uma entrada e uma saída)

SNLA

Stochastic Newton-like Algorithm (Algoritmo estocástico do tipo Newton)

SNR

Signal-to-Noise Ratio (Relação sinal-ruído)

$\mathrm{SOM}$

Self-Organized Maps (Mapas auto-organizáveis)

SOS

Second-Order Statistics (Estatísticas de segunda ordem)

SPE

Sem Propagação de Erros

SWA

Shalvi-Weinstein Algorithm (Algoritmo de Shalvi-Weinstein) 


\section{Lista de Símbolos}

Nesta tese, matrizes são indicadas por letras maiúsculas em negrito, por exemplo $\hat{\mathbf{R}}(n)$ e $\mathcal{H}$. Vetores coluna são indicados usando-se letras minúsculas em negrito, por exemplo $\mathbf{w}(n)$ e $\breve{\mathbf{c}}_{i}$. Escalares são representados por letras minúsculas ou maiúsculas em itálico, por exemplo $M$, $N_{h}, \mu$ e $y(n)$. Nos capítulos 2,3 e 4, a letra grega $\alpha$ pode representar um passo de adaptação que, dependendo do algoritmo, é um escalar ou uma matriz. Uma outra exceção são os símbolos $\boldsymbol{C}_{2,1,1}^{y, y^{*}, \mathbf{u}^{*}}$ e $\boldsymbol{C}\left(x^{p+q}, \mathbf{u}\right)$, que embora estejam representados por letras maiúsculas em negrito, indicam vetores coluna. A seguir, são listados os principais símbolos utilizados.

\section{Símbolos gerais}

$\mathbb{R} \quad$ conjunto dos números reais

$\mathbb{C} \quad$ conjunto dos números complexos

$(\cdot)^{T} \quad$ transposição de vetores ou matrizes

$(\cdot)^{*} \quad$ complexo conjugado

$(\cdot)^{H} \quad$ hermitiano (transposição do complexo conjugado) de vetores ou matrizes

valor estimado

$\mathrm{E}\{\cdot\} \quad$ operador esperança matemática

$\odot \quad$ operação de multiplicação elemento a elemento de vetores

$\operatorname{step}(\cdot) \quad$ função degrau

$\varphi(\cdot) \quad$ função não-linear

$z^{-1} \quad$ operador de atraso, $z^{-1} y(n)=y(n-1)$

$\|\mathbf{x}\| \quad$ norma euclidiana ou $\ell_{2}$ do vetor $\mathbf{x}$

$\|\mathbf{x}\|_{\mathbf{A}}^{2} \quad$ norma ao quadrado do vetor $\mathbf{x}$ ponderado pela matriz $\mathbf{A}$

$\operatorname{Tr}(\mathbf{A}) \quad$ traço (soma dos elementos da diagonal) da matriz A 


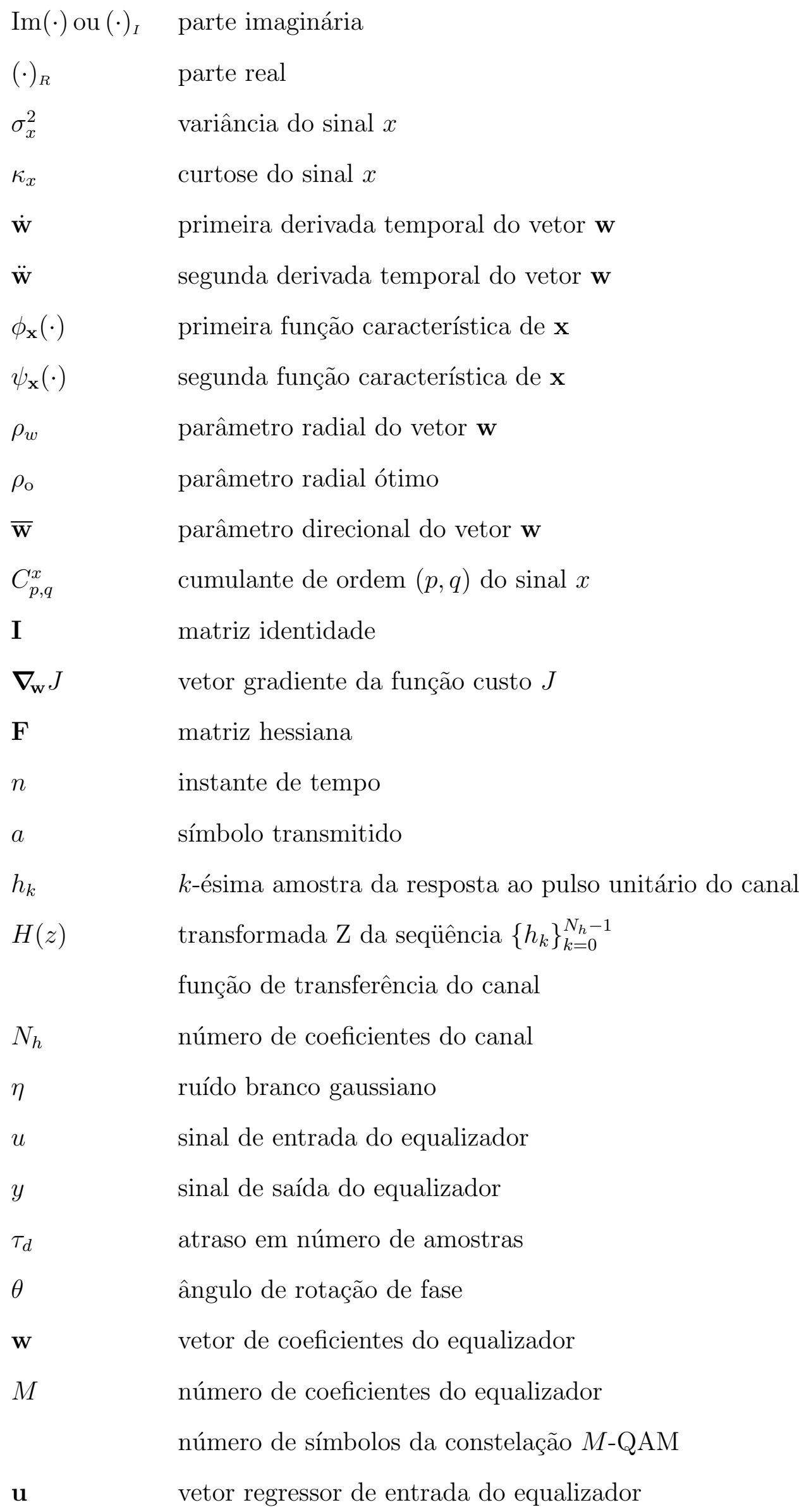



a
S
$\mathbf{s}_{z f}$
$\mathbf{s}_{e}$
$\mathcal{S}$
$\mathcal{H}$
$\mathcal{P}$
R
$S(z)$
$\beta$
$\beta_{S W}$
$J_{G}$
$\bar{J}_{G}$
$J_{S W}$
$R_{2}^{a}, \gamma^{a}, \gamma$
$R_{2}^{y}, \gamma^{y}$
$R_{2}^{a y}$
$\mu, \mu_{c}, \mu_{d}, \mu_{\theta}$
$\bar{\mu}$
$\rho$
$\bar{\rho}$
$f\left(\mathbf{s}_{n}\right)=f_{n}$
$N_{b}$
P
$\delta$
$\lambda, \lambda_{r}, \lambda_{f}$
$\mathbf{M}_{1}, \mathbf{M}_{2}, \mathbf{M}_{3}$
$\lambda_{L}$
$\mathbf{w}_{\mathrm{O}}$
q
vetor regressor da seqüência dos símbolos emitidos
vetor de resposta combinada da cascata canal-equalizador
vetor de resposta combinada - solução ótima de zero-forcing
vetor de resposta combinada num ponto estacionário
subespaço das respostas combinadas atingíveis
matriz de convolução do canal
operador de projeção ortogonal sobre $\mathcal{S}$
matriz de autocorrelação do sinal de entrada do equalizador
função de transferência global da cascata canal-equalizador
constante que distingue o caso complexo do real
passo de adaptação constante introduzido no SD-SWA
função custo de Godard
função custo de Godard reduzida
função custo de Shalvi-Weinstein
escalares que dependem das estatísticas do sinal $a$
escalares que dependem das estatísticas do sinal $y$
escalar que depende das estatísticas dos sinais $a$ e $y$
passos de adaptação
inverso da norma ao quadrado do vetor $\mathbf{u}$
passo de adaptação de algoritmos do tipo quase-Newton
inverso da norma ao quadrado do vetor $\mathbf{u}$ ponderado pela matriz $\mathbf{F}^{-1}$
função objetivo de Donoho
tamanho do bloco de dados para estimativa do gradiente
estimativa da matriz de autocorrelação inversa
constante de inicialização da matriz $\mathbf{P}$
fatores de esquecimento
matrizes da versão matricial do algoritmo "acelerador"
multiplicador de Lagrange
vetor de coeficientes ótimos
perturbação no vetor $\mathbf{w}_{\mathrm{o}}$ num ambiente não-estacionário 


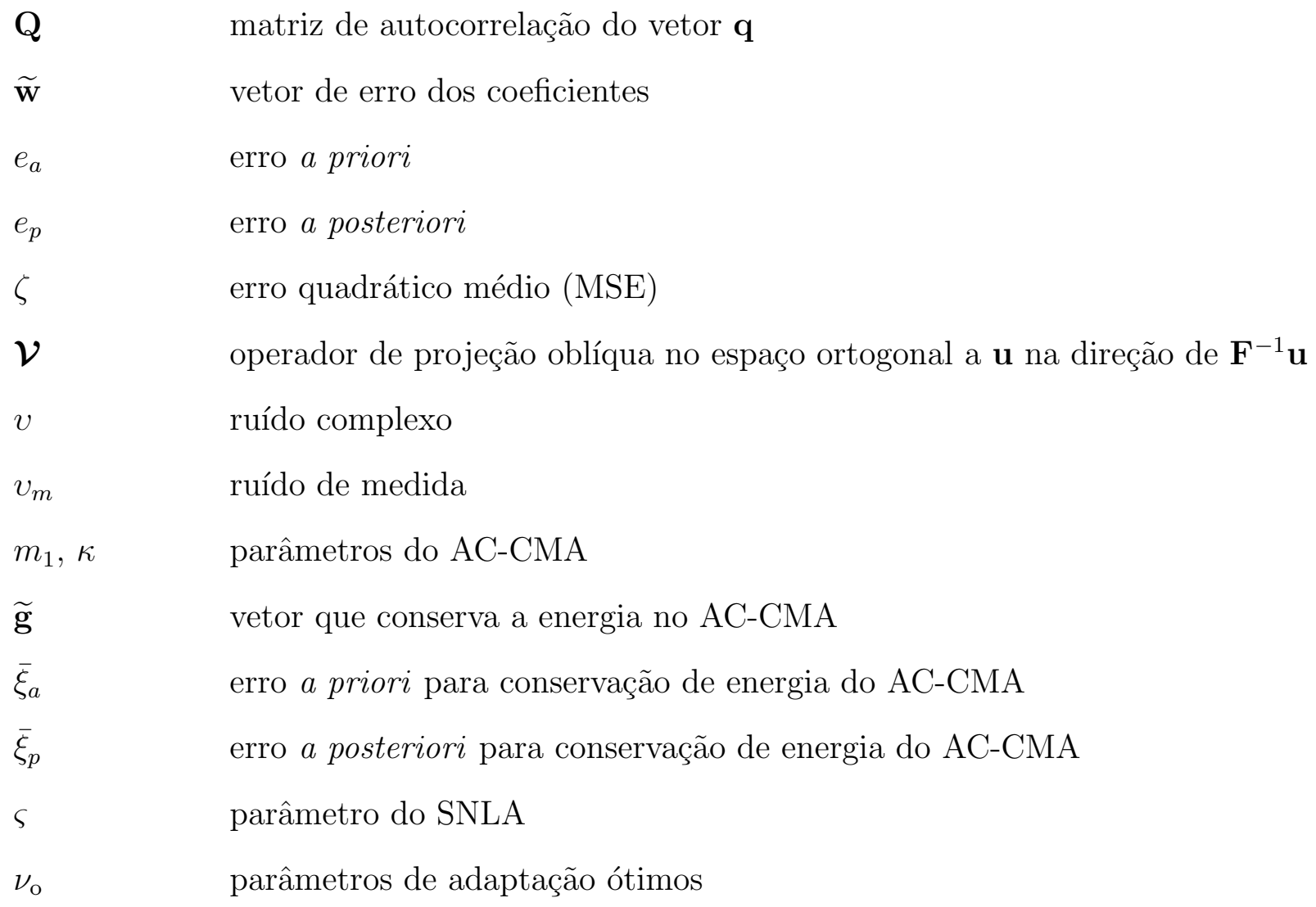

\section{Equalizador sobre-amostrado}

$L \quad$ fator de sobre-amostragem

$C_{i}(z) \quad$ função de transferência do subcanal $i$

$W_{i}(z) \quad$ função de transferência do subfiltro $i$ do equalizador

$\breve{\mathbf{c}_{k}}, \breve{\mathbf{w}_{m}} \quad$ vetores contendo coeficientes do canal e do equalizador

$\mathcal{C} \quad$ matriz de convolução do canal virtual

$M_{c} \quad$ número de coeficientes de cada subfiltro do equalizador

$N_{c} \quad$ número de coeficientes de cada subcanal

\section{Equalizador com realimentação de decisões}

$\begin{array}{ll}W_{f}(z) & \text { função de transferência do filtro direto } \\ W_{b}(z) & \text { função de transferência do filtro de realimentação } \\ y_{f} & \text { sinal de saída do filtro direto } \\ y_{b} & \text { sinal de saída do filtro de realimentação }\end{array}$




$\begin{array}{ll}M_{f} & \text { número de coeficientes do filtro direto } \\ M_{b} & \text { número de coeficientes do filtro de realimentação } \\ M_{s} & \text { dimensão do vetor de resposta combinada da cascata canal-filtro direto } \\ \mathbf{w}_{f} & \text { vetor de coeficientes do filtro direto } \\ \mathbf{w}_{b} & \text { vetor de coeficientes do filtro de realimentação } \\ \hat{\mathbf{a}}_{\tau_{d}} & \text { vetor regressor de entrada do filtro de realimentação } \\ \mathbf{s}_{f} & \text { vetor de resposta combinada da cascata canal-filtro direto } \\ \mathbf{w}_{f b} & \text { vetor contendo os coeficientes dos filtros direto e de realimentação } \\ \mathbf{u}_{f b} & \text { vetor contendo as entradas do filtros direto e de realimentação } \\ \mathcal{H}_{f b} & \text { matriz de convolução do canal, obtida da interpretação do DFE } \\ & \text { como um sistema SIMO } \\ C_{w} & \text { variável associada à restrição na função custo de Godard }\end{array}$

\section{Equalização espaço-temporal}

$\begin{array}{ll}a_{i} & \text { sinal da fonte (usuário) } i \\ N_{u} & \text { número de usuários } \\ M_{u} & \text { número de coeficientes de cada filtro do equalizador } \\ k_{1} & \text { inteiro associado ao espalhamento de atraso do canal } \\ K & \text { número de sensores (antenas) } \\ K_{t} & \text { inteiro associado à diversidade temporal } \\ K_{c} & \text { número de coeficientes da resposta ao pulso unitário de cada canal } \\ H_{i j}(z) & \text { função de transferência do canal que liga o usuário } i \text { à antena } j \\ \mathcal{H}_{i j} & \text { matriz de convolução do canal } H_{i j}(z) \\ r_{i k} & \text { correlação cruzada entre as saídas dos equalizadores } i \text { e } j \\ \hat{\mathbf{r}}_{i} & \text { vetor que contém as estimativas das correlações cruzadas entre } \\ \varrho & \text { o usuário } i \text { e os demais usuários } \\ \mathbf{y}_{i} & \text { fator de ponderação do termo de correlação cruzada } \\ & \text { vetor contendo amostras das saídas de todos os equalizadores, } \\ & \text { com exceção do } i \text {-ésimo }\end{array}$


$\bar{e}_{i} \quad$ erro para atualização dos coeficientes do $i$-ésimo equalizador

\section{Equalizador híbrido}

$M_{r} \quad$ número de coeficientes do filtro de realimentação

$M_{f} \quad$ número de entradas do filtro direto

$M_{b} \quad$ número de amostras realimentadas na RNN

$\chi(\cdot) \quad$ função de ativação do neurônio

$N \quad$ número de neurônios da RNN

$y_{\mathrm{RNN}} \quad$ saída da RNN e entrada do filtro de realimentação 


\section{Capítulo 1}

\section{Introdução e formulação do problema}

Neste capítulo, o contexto, as contribuições e a estrutura do trabalho são apresentados. Inicialmente, consideram-se os problemas da desconvolução autodidata e da separação autodidata de fontes. Em seguida, suas formulações são particularizadas para equalização de canais de comunicação nos casos mono e multiusuário. As condições de equalização perfeita para o equalizador linear transversal e para o que faz realimentação de decisões passadas são revisitadas. Revisitam-se também os métodos de projeto de algoritmos adaptativos. Finalmente, os objetivos, as contribuições e a estrutura do trabalho são apresentados.

\subsection{A desconvolução autodidata}

Um diagrama simplificado da desconvolução autodidata, também conhecida na literatura como Blind Deconvolution, é apresentado na Figura 1.1 e descrito a seguir. Deseja-se recuperar o sinal $a(n)$, entrada de um sistema desconhecido $H$, a partir do sinal de saída $u(n)$. Para isso, utiliza-se um filtro ajustável $W$ que idealmente deve convergir para o sistema inverso $H^{-1}$ [Shalvi \& Weinstein, 1994]. Esse problema aparece em diversas áreas, por exemplo, acústica, óptica, processamento de imagens médicas, geofísica, comunicações, controle etc. [Kofidis, 2001]. Em comunicações, o termo "equalização autodidata" é mais comum, já que o principal interesse é recuperar a seqüência de símbolos transmitida através de um canal de comunicação dispersivo. Em controle, a desconvolução autodidata é usualmente conhecida como identificação autodidata, sendo que se deseja identificar o modelo do sistema inverso. Em acústica, óptica e geofísica o termo desconvolução é mais adequado já 


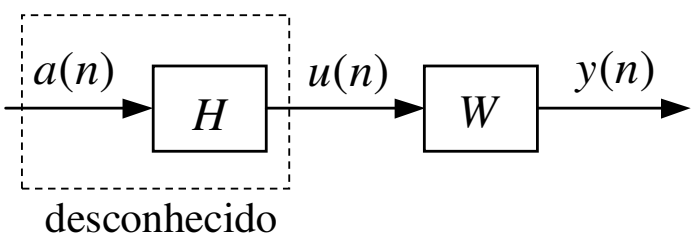

Figura 1.1: Diagrama simplificado do problema de desconvolução autodidata.

que o principal objetivo é "eliminar" a influência do sistema $H$, encontrando seu inverso estável.

Usualmente, o modelo do sistema $H$ inclui ruído aditivo e/ou não-linearidades, o que pode dificultar a solução do problema. Na ausência de ruído e assumindo que o sistema é linear, se $H$ é de fase mínima, a solução ótima é simplesmente a função de transferência inversa do sistema, isto é, $H^{-1}$. Neste caso, o sistema inverso é um filtro branqueador obtido a partir da teoria de predição linear [Haykin, 1996]. Em contrapartida, quando $H$ é de fase não-mínima e/ou há presença de ruído, o uso de um modelo inverso não é mais uma solução ótima [Haykin, 1996].

Vários métodos para resolver o problema de desconvolução autodidata em situações realistas foram propostos na literatura. Os métodos clássicos podem ser divididos em três grupos [Haykin, 2000]:

- métodos baseados em estatísticas de segunda ordem (SOS - Second-Order Statistics);

- métodos que utilizam estatísticas de ordem superior a dois (HOS - Higher Order Statistics);

- métodos baseados na teoria da informação.

Os métodos que utilizam SOS exploram características de cicloestacionariedade do sinal $u(n)$ para identificar o sistema $H$. Se $u(n)$ não for cicloestacionário, esses métodos não serão suficientes para resolver o problema de desconvolução de forma autodidata, dado que a autocorrelação de $u(n)$ não depende da fase do sistema $H$ [Shalvi \& Weinstein, 1994; Gardner \& Spooner, 1994]. Conseqüentemente, deve-se garantir que $u(n)$ seja cicloestacionário, o que pode ser feito através da técnica de sobre-amostragem [Tong et al., 1994; Slock, 1994; Moulines et al., 1995; Treichler et al., 1996]. Dentre os métodos que utilizam SOS, destacam- 
se os dos mínimos quadrados [Xu et al., 1995], os baseados em subespaços [Moulines et al., 1995] e os de predição linear [Slock, 1994]. Sob certas condições, esses métodos podem fazer a identificação perfeita do sistema [Mboup \& Regalia, 2000]. Tais condições incluem ausência de ruído e conhecimento da ordem exata, ou seja, o comprimento do identificador deve ser igual ao da parte significativa do sistema a ser identificado [Liavas et al., 2000]. Devido à necessidade da identificação exata da ordem do sistema, métodos que utilizam SOS podem apresentar um desempenho inadequado em condições realistas [Liavas et al., 2000; Mboup \& Regalia, 2000].

Os métodos que utilizam HOS são matematicamente fundamentados em conceitos de momentos e cumulantes de ordem elevada (superior a dois). Benveniste et al. (1980) mostraram que se os processos aleatórios y e $a$ possuem os mesmos momentos para todas as ordens, ou seja, $\mathrm{E}\left\{|y|^{k}\right\}=\mathrm{E}\left\{|a|^{k}\right\}$ para todo $k>0$, então suas funções densidade de probabilidade coincidem. Assim, o filtro $W$ deve ser ajustado para que os momentos de sua saída $y(n)$ sejam iguais aos momentos do sinal $a(n)$. Donoho (1981) e depois Shalvi e Weinstein (1990) propuseram um critério simplificado baseado em estatísticas de ordem quatro, mais especificamente na curtose do sinal $y(n)$. Em [Shalvi \& Weinstein, 1990], foi mostrado que para recuperar a seqüência original, é suficiente que as curtoses dos sinais $y(n)$ e $a(n)$ sejam iguais. Posteriormente em [Shalvi \& Weinstein, 1993, 1994], esse critério foi generalizado, utilizandose o cumulante de ordem $(p, q)$ do sinal $y(n)$ com $p$ e $q$ inteiros tal que $p+q>2$. Um outro critério bastante conhecido na literatura é o do Módulo Constante [Godard, 1980], que, sob certas circunstâncias, é equivalente ao de Shalvi-Weinstein [Shalvi \& Weinstein, 1994; Regalia, 1999]. A velocidade de convergência dos algoritmos baseados nesses critérios está diretamente ligada à qualidade das estimativas das estatísticas de ordem superior. Quando comparados aos algoritmos baseados em SOS, os algoritmos que utilizam HOS apresentam uma baixa velocidade de convergência. Isso justificou o estudo dos métodos SOS durante muito tempo [Liavas et al., 2000]. No entanto, foi verificado posteriormente que ao se utilizar a técnica de sobre-amostragem, os algoritmos HOS deixavam de ser lentos, podendo inclusive atingir a desconvolução perfeita na ausência de ruído [Treichler et al., 1996; Regalia \& Mboup, 1999; Ding \& Li, 2001]. Além disso, como o bom desempenho dos métodos SOS está ligado à identificação exata da ordem do sistema, os que são baseados em HOS passaram a 
ser considerados mais robustos em condições realistas [Mboup \& Regalia, 2000].

Finalmente, os métodos baseados na teoria da informação ajustam o filtro $W$ utilizando o sinal $u(n)$, para que a função densidade de probabilidade (f.d.p.) do sinal $y(n)$ seja igual à do sinal $a(n)$. Isso é equivalente a maximizar a informação mútua entre a seqüência original $a(n)$ e a saída atual do sistema, $y(n)$. Em outras palavras, ao se igualar as características estatísticas dos sinais $a(n)$ e $y(n)$, minimiza-se a entropia do erro (distância) entre essas duas seqüências [Erdogmus \& Principe, 2002]. A noção de entropia, introduzida por Shannon (1948), define uma medida de incerteza no espaço das distribuições de probabilidades, sendo que a distribuição que gera a máxima incerteza terá a máxima entropia [Lathi, 1998; Haykin, 1999]. Esse conceito foi primeiramente utilizado em desconvolução autodidata por Wiggins (1978) e Donoho (1981), que desenvolveram algoritmos baseados na minimização da entropia. No entanto, em aplicações de tempo real, uma estimativa eficiente da entropia de Shannon é relativamente complexa [Bercher \& Vignat, 2000; Santamaría et al., 2002a,b]. Desta forma, surgiram na literatura algoritmos de desconvolução autodidata baseados na minimização de critérios relacionados direta ou indiretamente com a entropia, e que são mais fáceis de estimar. Dentre esses algoritmos destacam-se os que utilizam a divergência (distância) de Kullback-Leibler para forçar igualdade das funções densidade de probabilidade [Karaoguz \& Adarlan, 1992; Chen et al., 1995; Cavalcante et al., 2003] e os que utilizam a entropia de Renyi [Santamaría et al., 2002a,b].

Grande parte das técnicas clássicas de desconvolução autodidata baseadas em HOS podem ser entendidas como métodos de minimização da entropia [Shalvi \& Weinstein, 1994; Regalia \& Mboup, 2001]. Ao se considerar por exemplo a igualdade dos cumulantes de segunda e quarta ordens dos sinais $a(n)$ e $y(n)$, é possível recuperar a seqüência original [Shalvi \& Weinstein, 1994]. Se isso ocorrer, a igualdade entre as funções densidade de probabilidade desses sinais é obtida e conseqüentemente a entropia é minimizada de forma indireta.

Um outro método clássico de estimação que também pode ser utilizado no problema de desconvolução autodidata é o da máxima verossimilhança (ML - Maximum Likelihood). Esse método é baseado na maximização do logaritmo da função de verossimilhança (loglikelihood function) que depende da f.d.p. do sinal $y(n)$ [Haykin, 1999]. Atinge-se a ML quando a f.d.p. de $y(n)$ é igual à f.d.p. de $a(n)$. Conseqüentemente, pode-se demonstrar que 
o critério da máxima verossimilhança é equivalente ao critério da mínima entropia [Cardoso, 1997, 1998; Haykin, 1999]. Como neste último, uma estimativa eficiente da função de ML pode exigir um elevado esforço computacional, o que não é interessante para aplicações de tempo real. Dada a equivalência entre esses métodos, os critérios baseados em HOS, como o critério de Shalvi-Weinstein por exemplo, também podem ser interpretados como métodos simplificados para maximização da função de verossimilhança. Inspirando-se em [Tong \& Perreau, 1998], um diagrama de blocos, ligando os métodos HOS com a minimização da entropia e a maximização da função de verossimilhança, é apresentado na Figura 1.2.

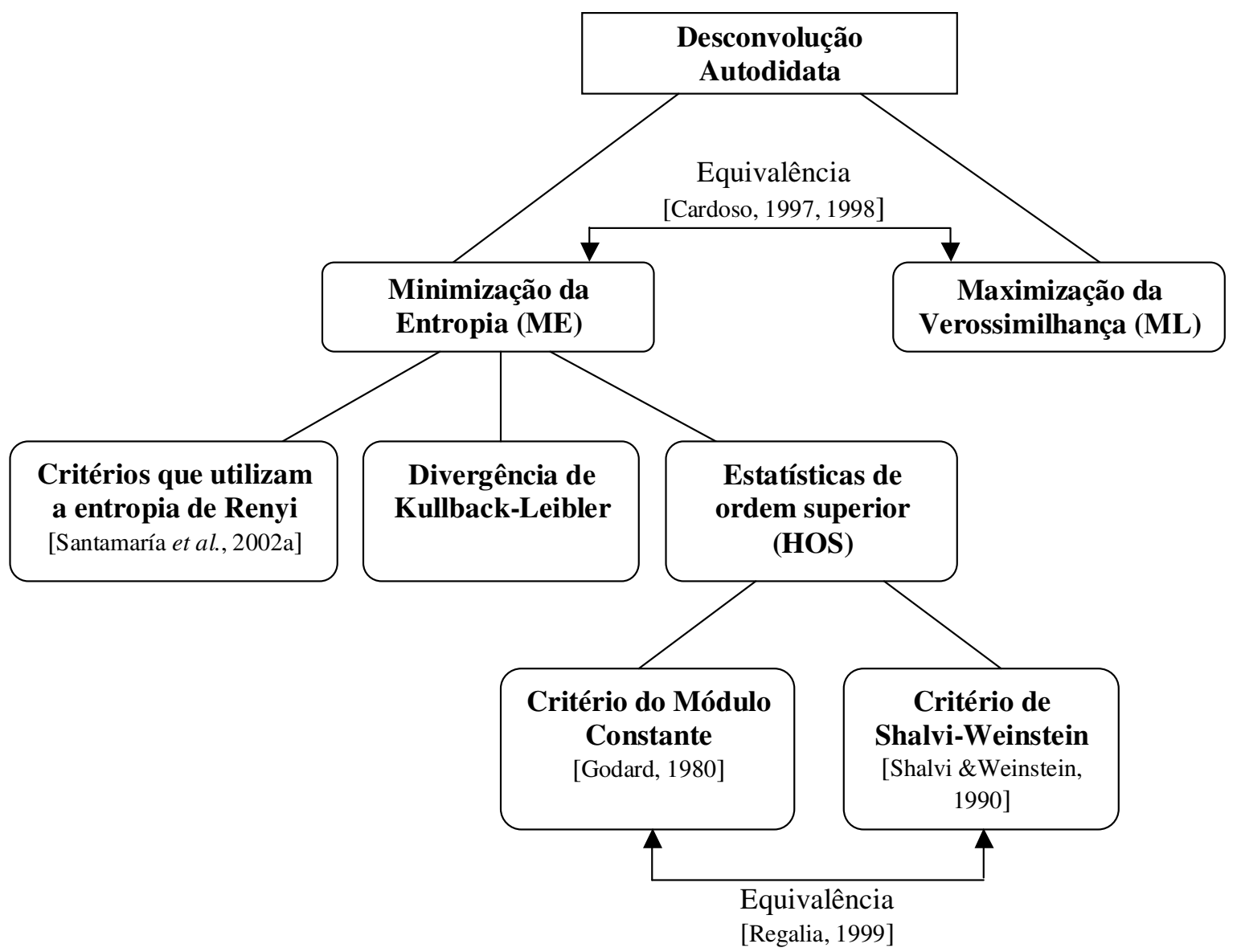

Figura 1.2: Relação entre os métodos HOS e os métodos minimização da entropia para desconvolução autodidata.

\subsection{A separação autodidata de fontes}

Uma extensão do problema de desconvolução autodidata é a separação autodidata de fontes também conhecida na literatura como Blind Source Separation (BSS), cujo esquema é mos- 
trado na Figura 1.3. O problema consiste na recuperação de $N_{u}$ sinais (fontes) a partir de $K$ sinais observáveis (misturas). Tipicamente os sinais $u_{j}, j=1,2, \ldots, K$ são obtidos na saída de um conjunto de $K$ sensores, sendo que cada sensor recebe uma combinação ponderada dos sinais $a_{i}, i=1,2, \ldots, N_{u}$, o que caracteriza a diversidade espacial [Paulraj \& Papadias, 1997; Escudero, 1998]. A fim de resolver esse problema de forma autodidata, assume-se independência estatística entre os sinais das fontes e o conhecimento da distribuição de probabilidades das entradas. Embora a consideração de independência seja uma hipótese "forte", ela é razoável em várias aplicações práticas [Cardoso, 1998]. Portanto, pode-se considerar o problema de BSS como uma extensão do problema de desconvolução autodidata para o caso MIMO (Multiple-Input Multiple-Output). A matriz H contém os coeficientes que misturam as fontes e a matriz $\mathbf{W}$ deve ser ajustada para que as saídas $y_{i}$, $i=1,2, \ldots, N_{u}$ convirjam para as fontes $a_{i}$. Em geral, isso é feito através da otimização de critérios baseados na teoria da informação e em estatísticas de ordem superior [Cardoso, 1998]. Os critérios de Shalvi-Weinstein e do Módulo Constante também podem ser utilizados em algumas aplicações de BSS, principalmente no processamento de sinais de comunicação [Castedo et al., 1997; Papadias \& Paulraj, 1997a; Papadias, 2000a,b]. Neste caso, devem-se penalizar as correlações entre os sinais de saída $y_{i}, i=1,2, \ldots, N_{u}$, para evitar convergência para mesma fonte.

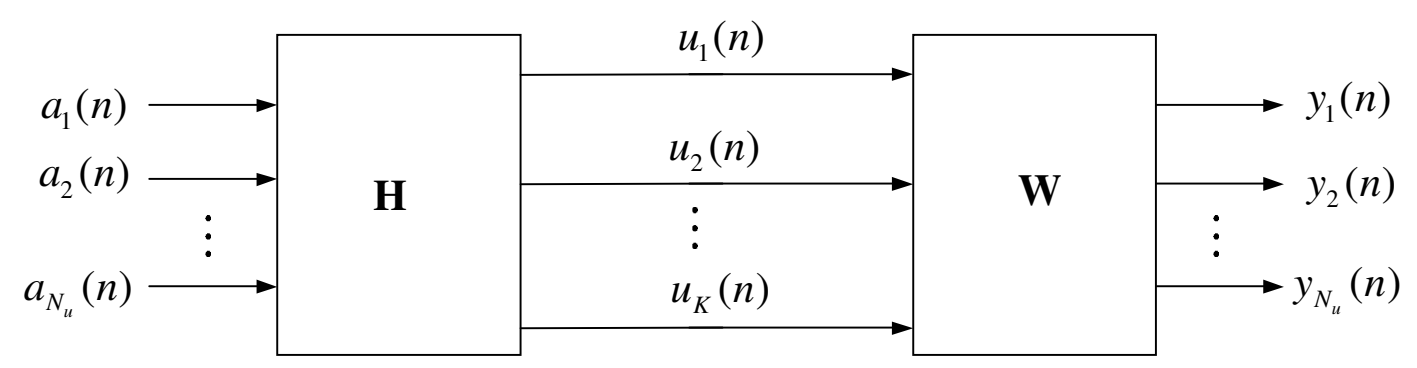

Figura 1.3: Diagrama simplificado do problema de separação de fontes.

Outro método utilizado em BSS é o chamado ICA (Independent Component Analysis). Ele tem sido explorado na literatura desde sua concepção por Comon (1994) [Haykin, 1999]. Esse método tem como objetivo transformar linearmente um vetor aleatório em outro com tantos componentes independentes quanto possível [Kofidis, 2001]. Sob condições ideais (i.e., BSS perfeita), o método de minimização da entropia e o ICA coincidem [Haykin, 1999]. 


\subsection{Formulação do problema - caso SISO}

A equalização de canais de comunicação é uma das principais aplicações da desconvolução autodidata. Neste caso, um equalizador adaptativo é utilizado no receptor para compensar as alterações do sinal, decorrentes do meio de transmissão. Considerando inicialmente o caso SISO e uma estrutura transversal para o equalizador, tem-se o diagrama de blocos da Figura 1.4. A seqüência transmitida $a(n)$ é em geral não-gaussiana, independente e identicamente distribuída (i.i.d.). O sistema $H$ representa não só o canal físico de transmissão, mas também o sistema de transmissão/modulação e o sistema de recepção/demodulação, efetivamente presentes em qualquer sistema de comunicação prático. Assim, denomina-se aqui como canal um modelo de tempo discreto para o sistema de transmissão, o canal físico e o sistema de recepção. Na maioria das aplicações, as distorções decorrentes do canal $H$ são bem modeladas por um filtro FIR (Finite Impulse Response). Neste caso, sua função de transferência é dada por

$$
H(z)=\sum_{k=0}^{N_{h}-1} h_{k} z^{-k}
$$

sendo $N_{h}$ o número de coeficientes do canal e $h_{k}$ os coeficientes que caracterizam o mesmo. O sinal $u(n)$ é obtido a partir da convolução entre a resposta ao pulso unitário do canal e a seqüência transmitida somada a um ruído, ou seja,

$$
u(n)=\sum_{k=0}^{N_{h}-1} h_{k} a(n-k)+\eta(n) .
$$

Usualmente o ruído $\eta(n)$ é assumido aditivo, branco e gaussiano (AWGN - Additive White Gaussian Noise) com média nula e variância $\sigma_{\eta}^{2}$. Considerando esse modelo, os seguintes aspectos devem ser considerados no problema de equalização:

- a interferência intersimbólica (ISI Intersymbol Interference) causada pela sobreposição dos símbolos recebidos. No modelo linear apresentado, o efeito da ISI é representado pela convolução entre a seqüência de símbolos transmitida e a resposta ao pulso unitário do canal. Esse efeito dispersivo é devido aos diferentes atrasos na propagação do sinal, também conhecido como multipercurso;

- a presença de ruído intrínseco a todo sistema de transmissão que afeta o desempenho do equalizador; 


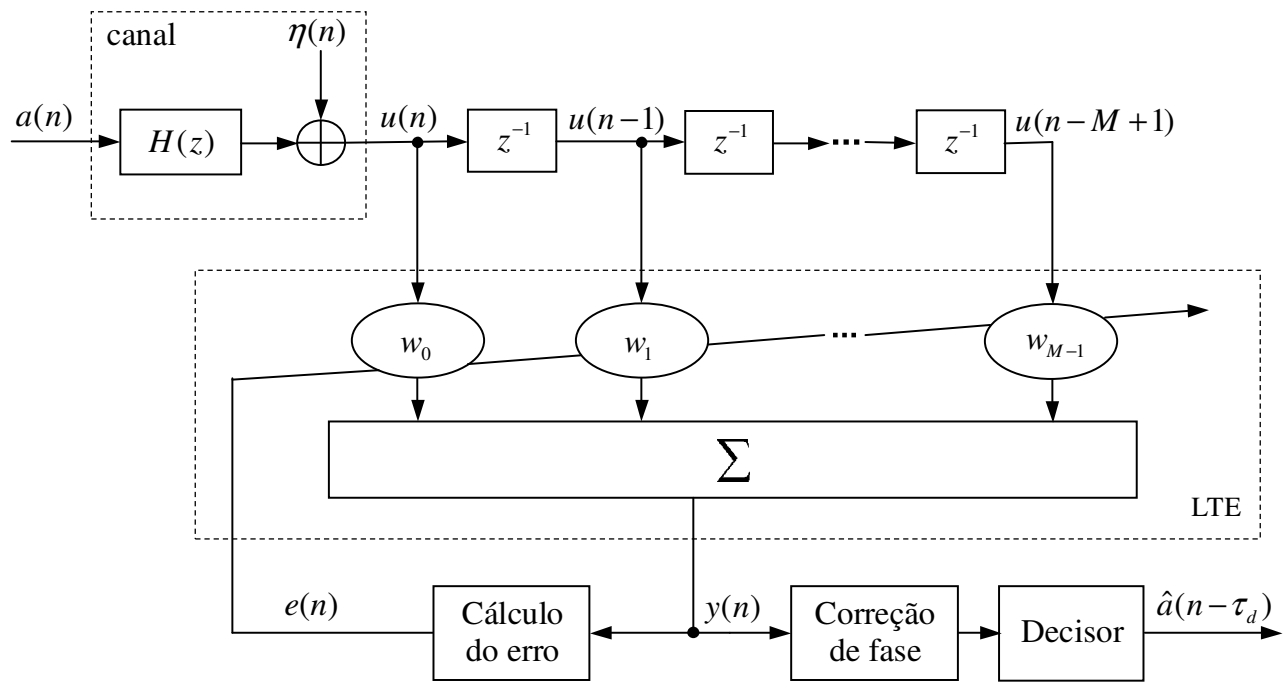

Figura 1.4: Esquema de um sistema de comunicação com o equalizador linear transversal (LTE) implementado de forma autodidata.

- o modelo do canal que pode ser de fase mínima, não-mínima e apresentar nãolinearidades. Além disso, ele ainda pode ser variante no tempo, como é o caso de canais com desvanecimento (fading).

Na Figura 1.5, é mostrado um exemplo do efeito do canal na transmissão de um sinal com 16-QAM e de sua possível recuperação por um equalizador autodidata. O equalizador deve ser ajustado para eliminar os efeitos do canal e recuperar a seqüência transmitida com um atraso $\tau_{d}$ e uma rotação de fase $\theta$ (Figura 1.5-c). Essa rotação deve ser corrigida antes do decisor, o que também pode ser feito de forma autodidata [Heidari \& Nasiri-Kenari, 2000; Proakis, 1996].

a)

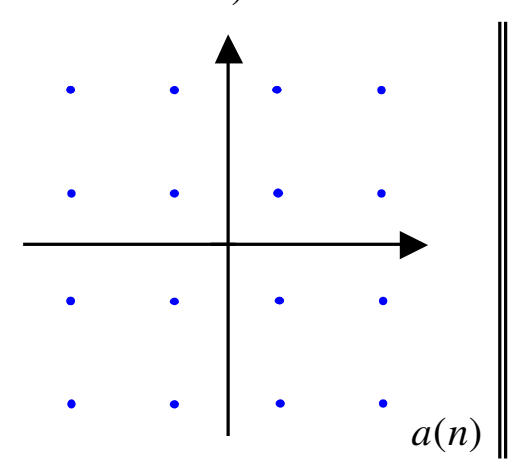

b)

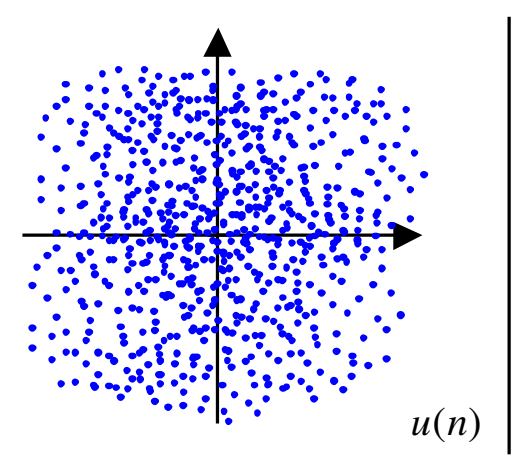

c)

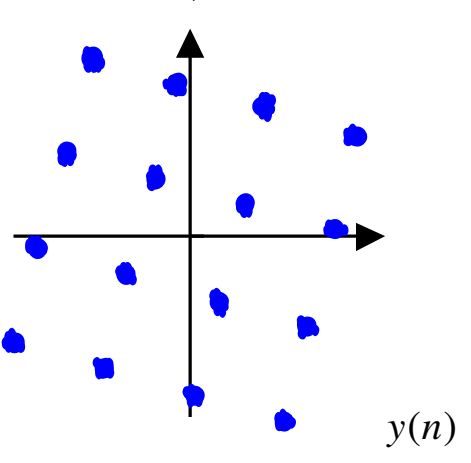

Figura 1.5: Exemplo do efeito do canal; a) Sinal transmitido com 16-QAM, b) sinal que chega no receptor e c) sinal de saída do equalizador autodidata. 
Considera-se o equalizador linear transversal (LTE - Linear Transversal Equalizer) da Figura 1.4 com os $M$ coeficientes agrupados no vetor

$$
\mathbf{w}=\left[\begin{array}{llll}
w_{0} & w_{1} & \cdots & w_{M-1}
\end{array}\right]^{T} .
$$

Os vetores regressores de entrada do equalizador e da seqüência de símbolos emitidos podem ser definidos respectivamente como

$$
\mathbf{u}(n)=\left[\begin{array}{llll}
u(n) & u(n-1) & \cdots & u(n-M+1)
\end{array}\right]^{T}
$$

e

$$
\mathbf{a}(n)=\left[\begin{array}{llll}
a(n) & a(n-1) & \cdots & a\left(n-N_{h}-M+2\right)
\end{array}\right]^{T} .
$$

Utilizando o modelo do canal da Equação (1.1), o mapeamento entre sua entrada e sua saída na ausência de ruído pode ser expresso como

$$
\underbrace{\left[\begin{array}{c}
u(n) \\
u(n-1) \\
\vdots \\
u(n-M+1)
\end{array}\right]}_{\mathbf{u}(n)}=\underbrace{\left[\begin{array}{ccccccc}
h_{0} & h_{1} & \cdots & h_{N_{h}-1} & 0 & \cdots & 0 \\
0 & h_{0} & \cdots & h_{N_{h}-2} & h_{N_{h}-1} & \ddots & \vdots \\
\vdots & \ddots & \ddots & \ddots & \ddots & \ddots & 0 \\
0 & \cdots & 0 & h_{0} & \cdots & h_{N_{h}-2} & h_{N_{h}-1}
\end{array}\right]}_{\mathcal{H}^{T}} \underbrace{\left[\begin{array}{c}
a(n) \\
a(n-1) \\
a\left(n-N_{h}-M+2\right)
\end{array}\right]}_{\mathbf{a}(n)}
$$

sendo $\mathcal{H}$ a matriz de convolução do canal. A saída do equalizador é dada por

$$
y(n)=\mathbf{u}^{T}(n) \mathbf{w}
$$

Observando (1.6) e denotando $\mathbf{s}=\mathcal{H} \mathbf{w}$, a saída do equalizador pode também ser expressa como

$$
y(n)=\mathbf{a}^{T}(n) \mathbf{s}=\sum_{k=0}^{N_{h}+M-2} s_{k} a(n-k) .
$$


O vetor s de dimensão $N_{h}+M-1$,

$$
\underbrace{\left[\begin{array}{c}
s_{0} \\
s_{1} \\
\vdots \\
s_{M-1} \\
\vdots \\
s_{N_{h}+M-2}
\end{array}\right]}_{\mathbf{s}}=\underbrace{\left[\begin{array}{cccc}
h_{0} & 0 & \cdots & 0 \\
h_{1} & h_{0} & \ddots & \vdots \\
\vdots & \ddots & \ddots & 0 \\
h_{M-1} & \cdots & h_{1} & h_{0} \\
h_{M} & h_{M-1} & \cdots & h_{1} \\
\vdots & \ddots & \ddots & \vdots \\
0 & \cdots & 0 & h_{N_{h}-1}
\end{array}\right]}_{\mathcal{H}} \underbrace{\left[\begin{array}{c}
w_{0} \\
w_{1} \\
\vdots \\
w_{M-1}
\end{array}\right]}_{\mathbf{w}}
$$

representa a resposta combinada da cascata canal-equalizador e mapeia a seqüência de símbolos transmitidos $a(n)$ à seqüência de saída do equalizador $y(n)$ na ausência de ruído.

A equalização perfeita (condição de zero-forcing) ocorre quando a resposta combinada tiver a forma



sendo $\tau_{d}$ o número de amostras atrasadas e $\theta$ uma rotação de fase.

Da Equação (1.9), conclui-se que o vetor s pertence ao espaço formado pelas colunas da matriz de convolução do canal. Em outras palavras, a resposta combinada se restringe à ordem do espaço-imagem da matriz $\mathcal{H}$. Denotando esse espaço por $\mathcal{S}$ (subespaço de respostas combinadas atingíveis), o operador de projeção ortogonal sobre $\mathcal{S}$ é dado por $\mathcal{P}=\mathcal{H}\left(\mathcal{H}^{H} \mathcal{H}\right)^{\sharp} \mathcal{H}^{H}$, sendo que $\sharp$ denota a operação de pseudo-inversão [Regalia \& Mboup, 1999]. Se $\mathcal{P}=\mathbf{I}$, qualquer resposta combinada é atingível. Esse caso é conhecido como o da ordem suficiente. Em contrapartida, se $\mathcal{P} \neq \mathbf{I}$, somente uma parte do espaço $\mathcal{S}$ é alcançável, variando-se os coeficientes do equalizador. Esse caso é conhecido como submodelado [Regalia \& Mboup, 1999].

Além do operador de projeção, define-se também a matriz de autocorrelação da seqüência de entrada do equalizador

$$
\mathbf{R}=\mathrm{E}\left\{\mathbf{u}^{*}(n) \mathbf{u}^{T}(n)\right\}
$$

Na ausência de ruído e assumindo um canal invariante, têm-se $\mathbf{u}(n)=\mathcal{H}^{T} \mathbf{a}(n)$ e

$$
\mathbf{R}=\mathcal{H}^{H} \mathrm{E}\left\{\mathbf{a}^{*}(n) \mathbf{a}^{T}(n)\right\} \mathcal{H}
$$


No caso em que $a(n)$ é uma seqüência i.i.d., pode-se escrever ainda

$$
\mathbf{R}=\mathrm{E}\left\{|a(n)|^{2}\right\} \mathcal{H}^{H} \mathcal{H}
$$

Portanto, o condicionamento (razão entre os autovalores mínimo e máximo) de $\mathbf{R}$ depende diretamente das características da matriz $\mathcal{H}$. Quando o canal é ideal e $a(n)$ é i.i.d., $u(n)$ também será i.i.d. Neste caso, R é diagonal e seu condicionamento é igual a 1. Em contrapartida, se o canal tiver nulos espectrais profundos, o sinal $u(n)$ não será bem condicionado, o que torna o determinante de $\mathbf{R}$ próximo de zero, dificultando a operação de inversão em precisão finita [Haykin, 1996]. Quando $\mathbf{R}$ é não-singular, $\mathbf{R}^{-1}=\mathbf{R}^{\sharp}$ e o operador de projeção se reduz a

$$
\mathcal{P}=\mathcal{H}\left(\mathcal{H}^{H} \mathcal{H}\right)^{-1} \mathcal{H}^{H}
$$

substituindo-se a operação de pseudo-inversão pela operação de inversão [Stewart, 1973].

\subsubsection{Condições para equalização perfeita}

Assumindo o modelo do canal da Equação (1.1) e o equalizador linear transversal operando na taxa de transmissão dos símbolos, a equalização perfeita só poderá ser obtida na ausência de ruído e se o comprimento do equalizador for infinito. Para se obter equalização perfeita, utilizando-se um equalizador com resposta ao pulso unitário finita (FIR), deve-se considerar a técnica de sobre-amostragem [Tong et al., 1994; Slock, 1994; Moulines et al., 1995; Treichler et al., 1996], revisitada no Apêndice A. Neste caso, o equalizador opera numa taxa superior à dos símbolos, o que permite alterar a representação da matriz de convolução do canal, possibilitando obter $\mathcal{P}=\mathbf{I}$. O problema de equalização pode então passar da situação de submodelagem para a de ordem suficiente. No entanto, isso só ocorre na ausência de ruído pois, caso contrário, a equalização perfeita não é mais possível.

Considerando um fator de sobre-amostragem $L$ e ausência de ruído, tem-se o modelo equivalente de um sistema de comunicação mostrado na Figura 1.6. As funções de transferência dos subcanais são dadas por

$$
C_{i}(z)=\sum_{k=0}^{N_{c}-1} h_{i+k L} z^{-k},
$$

para $i=0,1, \cdots, L-1$, sendo $N_{c}=N_{h} / L$ o número de coeficientes de cada subcanal. Neste caso, assume-se sem perda de generalidade que o comprimento da resposta ao pulso 
unitário do canal é múltiplo inteiro do fator de sobre-amostragem. Cabe observar que $h_{k}$ com $k=0,1, \ldots, N_{h}-1$ representam os coeficientes da resposta ao pulso unitário do canal, obtidos da sobre-amostragem de um modelo de tempo contínuo $h(t)$, com taxa $L$ vezes maior que a de símbolos. Utilizando (1.15), a função de transferência do canal $H(z)$ pode ser expressa como

$$
H(z)=\sum_{i=0}^{L-1} z^{-i} C_{i}\left(z^{L}\right) .
$$

Definindo-se as funções de transferência dos subfiltros do equalizador como

$$
W_{i}(z)=\sum_{m=0}^{M_{c}-1} w_{m i} z^{-m},
$$

para $i=0,1, \cdots, L-1$, sendo $M_{c}$ o número de coeficientes de cada subfiltro do equalizador, a função de transferência global da cascata canal-equalizador é dada por

$$
S(z)=\sum_{i=0}^{L-1} C_{i}(z) W_{i}(z) .
$$

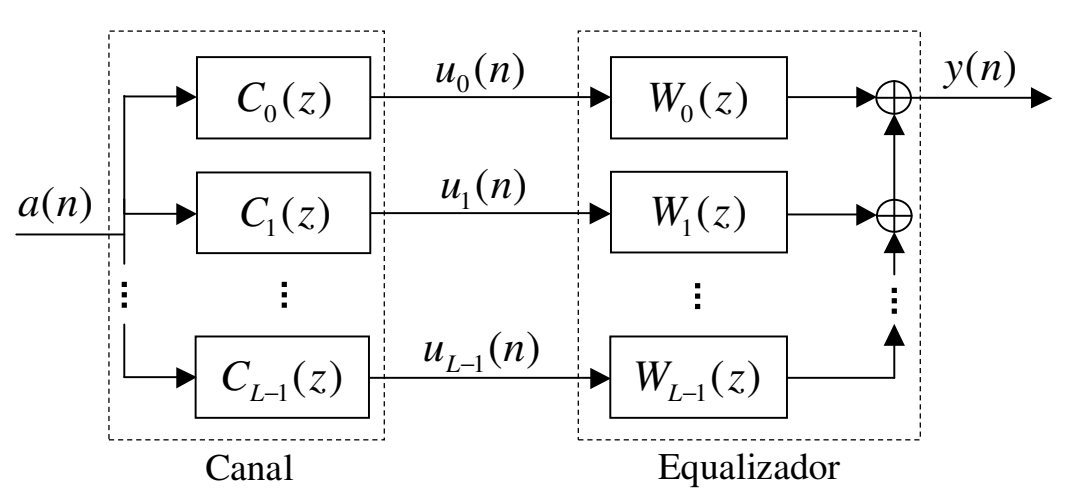

Figura 1.6: Modelo equivalente de um sistema de comunicação com equalizador sobre-amostrado por um fator $L$.

Definido-se os vetores

$$
\breve{\mathbf{c}}_{k}=\left[\begin{array}{llll}
h_{k L} & h_{k L+1} & \cdots & h_{(k+1) L-1}
\end{array}\right]^{T},
$$

com $k=0,1, \ldots, N_{c}-1 \mathrm{e}$

$$
\breve{\mathbf{w}}_{m}=\left[\begin{array}{llll}
w_{m 0} & w_{m 1} & \cdots & w_{m(L-1)}
\end{array}\right]^{T},
$$


com $m=0,1,2, \ldots, M_{c}-1$, o vetor de resposta combinada da cascata canal-equalizador pode ser expresso como

$$
\underbrace{\left[\begin{array}{c}
s_{0} \\
s_{1} \\
s_{M_{c}-1} \\
s_{M_{c}} \\
\vdots \\
s_{N_{c}+M_{c}-2}
\end{array}\right]}_{\mathbf{s}}=\underbrace{\left[\begin{array}{cccc}
\breve{\mathbf{c}}_{0}^{T} & \mathbf{0}_{1 \times L} & \cdots & \mathbf{0}_{1 \times L} \\
\breve{\mathbf{c}}_{1}^{T} & \breve{\mathbf{c}}_{0}^{T} & \ddots & \vdots \\
\vdots & \ddots & \ddots & \mathbf{0}_{1 \times L} \\
\breve{\mathbf{c}}_{M_{c}-1}^{T} & \ldots & \breve{\mathbf{c}}_{1}^{T} & \breve{\mathbf{c}}_{0}^{T} \\
\breve{\mathbf{c}}_{M_{c}}^{T} & \breve{\mathbf{c}}_{M_{c}-1}^{T} & \ldots & \breve{\mathbf{c}}_{1}^{T} \\
\vdots & \ddots & \ddots & \vdots \\
\mathbf{0}_{1 \times L} & \ldots & \mathbf{0}_{1 \times L} & \breve{\mathbf{c}}_{N_{c}-1}^{T}
\end{array}\right]}_{\mathbf{C}} \underbrace{\left[\begin{array}{c}
\breve{\mathbf{w}}_{0} \\
\breve{\mathbf{w}}_{1} \\
\vdots \\
\breve{\mathbf{w}}_{M_{c}-1}
\end{array}\right]}_{\mathbf{w}} .
$$

Cabe ressaltar que $\mathcal{C}_{\left(N_{c}+M_{c}-1\right) \times L M_{c}}$ é a matriz de convolução do "canal virtual" quando se considera sobre-amostragem por um fator $L$. O vetor de resposta combinada pertence ao espaço-imagem de $\mathcal{C}$, formado pelas colunas da matriz de convolução do canal virtual. Essa é a mesma interpretação do caso anterior, a diferença está apenas na matriz do canal que é "alterada" pela sobre-amostragem.

Uma condição suficiente, mas não necessária para se obter a equalização perfeita é dada por $\mathcal{P}=\mathbf{I}$. Para isso, as seguintes condições devem ser satisfeitas [Tong et al., 1994; Slock, 1994; Moulines et al., 1995; Li \& Ding, 1996; Regalia, 1998; Regalia \& Mboup, 1999]:

i) o número de colunas da matriz de convolução deve ser maior ou igual ao seu número de linhas

$$
L M_{c} \geq N_{c}+M_{c}-1 \Rightarrow M_{c} \geq \frac{N_{c}-1}{L-1}
$$

ii) o número de colunas linearmente independentes da matriz de convolução deve ser igual a $N_{c}+M_{c}-1$

iii) as funções de transferência polinomiais dos subcanais $C_{0}(z), \ldots, C_{L-1}(z)$ devem ser primas entre si.

As condições (i) e (ii) estão relacionadas com o espaço-imagem da matriz de convolução $\mathcal{C}$. O vetor de resposta combinada $\mathbf{s}$ tem dimensão $N_{c}+M_{c}-1$ e pertence ao espaço complexo de dimensão finita $\mathbb{C}^{N_{c}+M_{c}-1}$ (ou ao espaço $\mathbb{R}^{N_{c}+M_{c}-1}$ se os subcanais e subfiltros do equalizador forem ambos reais) [Regalia, 1999]. Em geral, o espaço-imagem da matriz $\mathcal{C}$ é um subespaço 
de $\mathbb{C}^{N_{c}+M_{c}-1}$. Assegurando-se as condições (i) e (ii), o espaço-imagem de $\mathcal{C}$ passa a ser igual ao espaço total $\mathbb{C}^{N_{c}+M_{c}-1}$. Neste caso, se (iii) também for satisfeita, a condição ótima de zero-forcing pode ser atingida, ajustando-se adequadamente os coeficientes dos subfiltros do equalizador. A condição (iii) está relacionada com a identidade de Bezout, revisitada a seguir [Kailath, 1980; Regalia, 1998].

Identidade de Bezout: As funções de transferência polinomiais $C_{0}(z), \ldots, C_{L-1}(z)$ serão primas entre si se e somente se existirem $W_{0}(z), \ldots, W_{L-1}(z)$, tais que

$$
S(z)=\sum_{i=0}^{L-1} C_{i}(z) W_{i}(z)=1, \quad \text { para todo } z .
$$

Considerando um atraso de $\tau_{d}$ amostras, a identidade de Bezout pode ser reescrita como [Roy, 2000]

$$
z^{-\tau_{d}}=\sum_{i=0}^{L-1} C_{i}(z) W_{i}(z) .
$$

Para satisfazer a condição (iii), os polinômios dos subcanais não devem ter zeros em comum. Se eles tiverem um zero em comum por exemplo, um polinômio comum $G(z)=g_{0}+g_{1} z^{-1}$ pode ser fatorado. Considerando

$$
C_{i}(z)=G(z) \bar{C}_{i}(z)
$$

a Equação (1.23) pode ser reescrita como

$$
\begin{aligned}
z^{-\tau_{d}} & =\left(g_{0}+g_{1} z^{-1}\right) \sum_{i=0}^{L-1} \bar{C}_{i}(z) W_{i}(z) \\
& =\left(g_{0}+g_{1} z^{-1}\right) \bar{S}(z)
\end{aligned}
$$

Essa equação resulta numa contradição porque nenhum polinômio finito $\bar{S}(z)$ quando multiplicado por $G(z)$ pode resultar no atraso $z^{-\tau_{d}}$, já que

$$
\bar{S}(z)=\frac{z^{-\tau_{d}}}{\left(g_{0}+g_{1} z^{-1}\right)}
$$

é um sistema do tipo IIR (Infinite Impulse Response) [Roy, 2000]. Além disso, se todos os polinômios dos subcanais não forem primos entre si, eles terão pelo menos um zero comum, denotado por $z_{0}$, tal que $C_{0}\left(z_{0}\right)=C_{1}\left(z_{0}\right)=\ldots=C_{L-1}\left(z_{0}\right)=0$. Neste caso, a função de transferência global do canal $H(z)$ terá $L$ zeros distribuídos numa circunferência de raio $\sqrt[L]{\left|z_{0}\right|}$. Uma pequena perturbação nos coeficientes de $H(z)$ modificará seus zeros, 
destruindo essa configuração particular, o que mostra que a condição (iii) é bastante realista, principalmente quando o canal é variante no tempo [Regalia, 1998].

Se as condições (i) e (iii) forem satisfeitas, a condição (ii) também será, pois pode-se mostrar neste caso que o posto da matriz de convolução é $N_{c}+M_{c}-1$ [Li \& Ding, 1996; Regalia, 1998; Kailath et al., 2000].

Cabe observar que para se ter equalização perfeita quando $L=1$ (sem sobre-amostragem), a ordem do equalizador deve ser infinita. Isso significa que não é possível encontrar um equalizador do tipo FIR que elimina completamente o efeito de um canal também do tipo FIR, sem a utilização da sobre-amostragem.

Para exemplificar, considera-se um canal de rádio digital, cuja resposta impulsiva finita $h(t)$ é amostrada na taxa de símbolos $(L=1)$ e numa taxa duas vezes maior $(L=2)$ [SPIB, 2001, chan4.mat]. Nas figuras 1.7 e 1.8, o módulo do operador de projeção $\mathcal{P}$ é apresentado em duas situações distintas. Na primeira, considera-se um caso submodelado, em que se utiliza a resposta ao pulso unitário do canal, obtida na taxa de símbolos e na segunda, um caso de ordem suficiente, em que se assume sobre-amostragem com $L=2$. É possível verificar que com a sobre-amostragem e uma escolha adequada dos comprimentos dos filtros do equalizador, as condições (i) a (iii) podem ser satisfeitas. Se isso ocorrer, $\mathcal{P}=\mathbf{I}$ e a equalização perfeita poderá ser atingida na ausência de ruído.

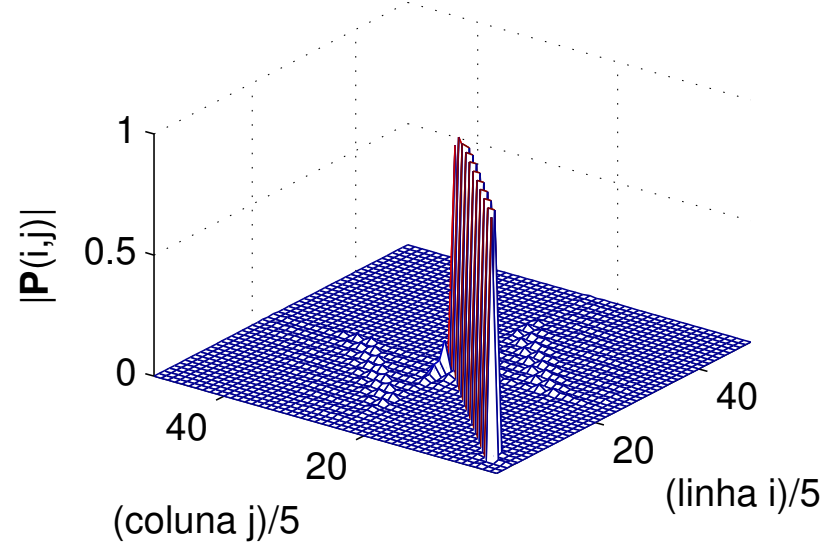

Figura 1.7: Módulo do operador de projeção $\mathcal{P}$ para o canal de rádio digital da referência [SPIB, 2001, chan4.mat]; $L=1, N_{c}=N_{h}=150$ e $M_{c}=100$. 


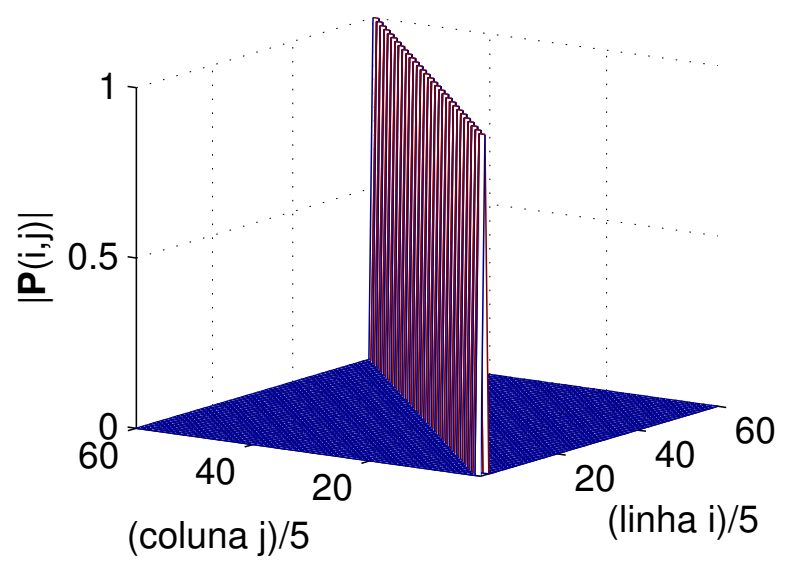

Figura 1.8: Módulo do operador de projeção $\mathcal{P}$ para o canal de rádio digital da referência [SPIB, 2001, chan4.mat]; $L=2, N_{c}=N_{h} / 2=150$ e $M_{c}=150$.

\subsubsection{O equalizador com realimentação de decisões}

Assumindo operação na taxa de símbolos (sem sobre-amostragem), o LTE pode apresentar um desempenho insatisfatório em muitas situações práticas, como canais esparsos, nãolineares e os que possuem nulos espectrais pronunciados [Bouchired et al., 1999; Silva, 2001; Rontogiannis \& Berberidis, 2003]. Cabe ressaltar ainda que, ao se considerar sobreamostragem, a equalização perfeita com o LTE só é possível para canais lineares, desde que as condições (i) a (iii) da Seção 1.3.1 sejam satisfeitas. Diante disso, o uso de estruturas não-lineares para equalização tem despertado um grande interesse na literatura [Kechriotis et al., 1994a; Bouchired et al., 1999; Ibnkahla, 2000; Silva, 2001] e pode apresentar um desempenho superior ao do LTE em diversas situações.

Dentre as estruturas não-lineares, a mais simples e utilizada na prática é a do equalizador que faz realimentação de decisões passadas (DFE -Decision Feedback Equalizer), cujo diagrama de blocos é mostrado na Figura 1.9. A realimentação de decisões faz com que o DFE tenha uma estrutura em que a não-linearidade do decisor fica posicionada numa malha de realimentação, formando um sistema não-linear [Qureshi, 1985; Proakis, 1996]. Considerando decisões corretas, o DFE pode ser interpretado como um equalizador sobreamostrado e, conseqüentemente, é possível atingir a equalização perfeita sob certas condições [Papadias \& Paulraj, 1995]. Comparando com o LTE, o DFE pode equalizar perfeitamente o canal na ausência de ruído, sem sobre-amostragem e com um número menor de coefi- 
cientes [Casas et al., 1995]. Além disso, não é necessário garantir que os subcanais sejam primos entre si [Papadias \& Paulraj, 1995]. Um outro aspecto importante é que o filtro direto do DFE também pode operar numa taxa superior à taxa de símbolos, ou seja, com sobre-amostragem. Neste caso, ele pode apresentar um desempenho superior ao do LTE sobre-amostrado [Szczecinski \& Gei, 2002]. A formulação matemática do DFE é considerada a seguir.

Na Figura 1.9, é mostrado um diagrama de blocos do DFE. Assume-se que as respostas ao pulso unitário dos filtros direto e de realimentação são finitas (FIR) e contêm respectivamente $M_{f}$ e $M_{b}$ coeficientes. O sinal recebido $u(n)$ entra no filtro direto $W_{f}(z)$ e as decisões passadas são realimentadas através do filtro de realimentação $W_{b}(z)$. O sinal resultante da combinação linear das saídas dos filtros, ou seja, $y(n)=y_{f}(n)+y_{b}(n)$ entra no decisor. Neste caso, considera-se que não há rotação de fase.

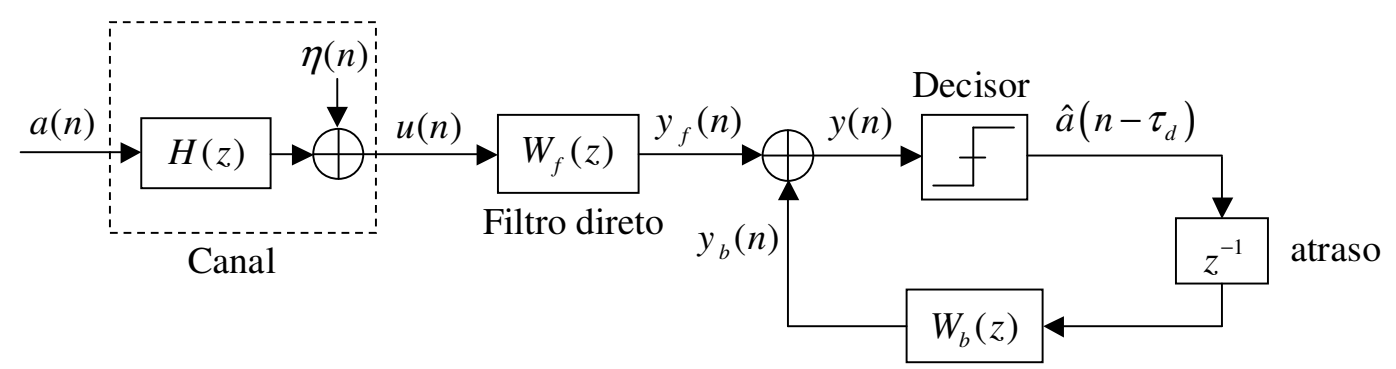

Filtro de realimentação

Figura 1.9: Diagrama de blocos do DFE.

O vetor regressor de entrada e o vetor de coeficientes do filtro direto são definidos como

$$
\mathbf{u}(n)=\left[\begin{array}{llll}
u(n) & u(n-1) & \cdots & u\left(n-M_{f}+1\right)
\end{array}\right]^{T}
$$

e

$$
\mathbf{w}_{f}=\left[\begin{array}{llll}
w_{f, 0} & w_{f, 1} & \cdots & w_{f, M_{f}-1}
\end{array}\right]^{T} .
$$

No caso do filtro de realimentação, o vetor de entrada e o vetor de coeficientes são dados por

$$
\hat{\mathbf{a}}_{\tau_{d}}(n)=\left[\begin{array}{llll}
\hat{a}\left(n-\tau_{d}-1\right) & \hat{a}\left(n-\tau_{d}-2\right) & \cdots & \hat{a}\left(n-\tau_{d}-M_{b}\right)
\end{array}\right]^{T}
$$

e

$$
\mathbf{w}_{b}=\left[\begin{array}{llll}
w_{b, 1} & w_{b, 2} & \cdots & w_{b, M_{b}}
\end{array}\right]^{T} .
$$


Com essas definições, a saída do DFE pode ser escrita como

$$
y(n)=\underbrace{\mathbf{u}^{T}(n) \mathbf{w}_{f}}_{y_{f}(n)}+\underbrace{\hat{\mathbf{a}}_{\tau_{d}}^{T}(n) \mathbf{w}_{b}}_{y_{b}(n)} .
$$

O vetor de resposta combinada da cascata canal-filtro direto é definido como

$$
\left[s_{f, 0} s_{f, 1} \cdots s_{f, M_{s}-1}\right]^{T}=\mathbf{s}_{f}=\mathcal{H} \mathbf{w}_{f}
$$

sendo $M_{s}=N_{h}+M_{f}-1$ a dimensão do vetor $\mathbf{s}_{f}$ e $\mathcal{H}$ a matriz de convolução do canal, definida em (1.6).

A seguir, partindo da interpretação do DFE como um equalizador sobre-amostrado e assumindo decisões corretas e ausência de ruído, as condições de equalização perfeita são obtidas.

\section{Condições para equalização perfeita com o DFE}

Concatenando os vetores de entrada e os vetores de coeficientes dos filtros direto e de realimentação do DFE nos vetores

$$
\mathbf{u}_{f b}(n)=\left[\begin{array}{ll}
\mathbf{u}^{T}(n) & \hat{\mathbf{a}}_{\tau_{d}}^{T}(n)
\end{array}\right]^{T}
$$

e

$$
\mathbf{w}_{f b}=\left[\begin{array}{cc}
\mathbf{w}_{f}^{T} & \mathbf{w}_{b}^{T}
\end{array}\right]^{T},
$$

a saída do DFE pode ser escrita como

$$
y(n)=\mathbf{u}_{f b}^{T}(n) \mathbf{w}_{f b} .
$$

Na Figura 1.10, é mostrado um modelo de um sistema de comunicação com DFE, assumindo-se decisões corretas. Neste caso, o vetor $\mathbf{u}_{f b}(n)$ pode ser interpretado como a saída de um sistema SIMO (Single-Input Multiple-Output) com uma entrada e duas saídas, descrito pelos subcanais

$$
H_{1}(z)=H(z) \quad \text { e } \quad H_{2}(z)=z^{-\tau_{d}-1} .
$$




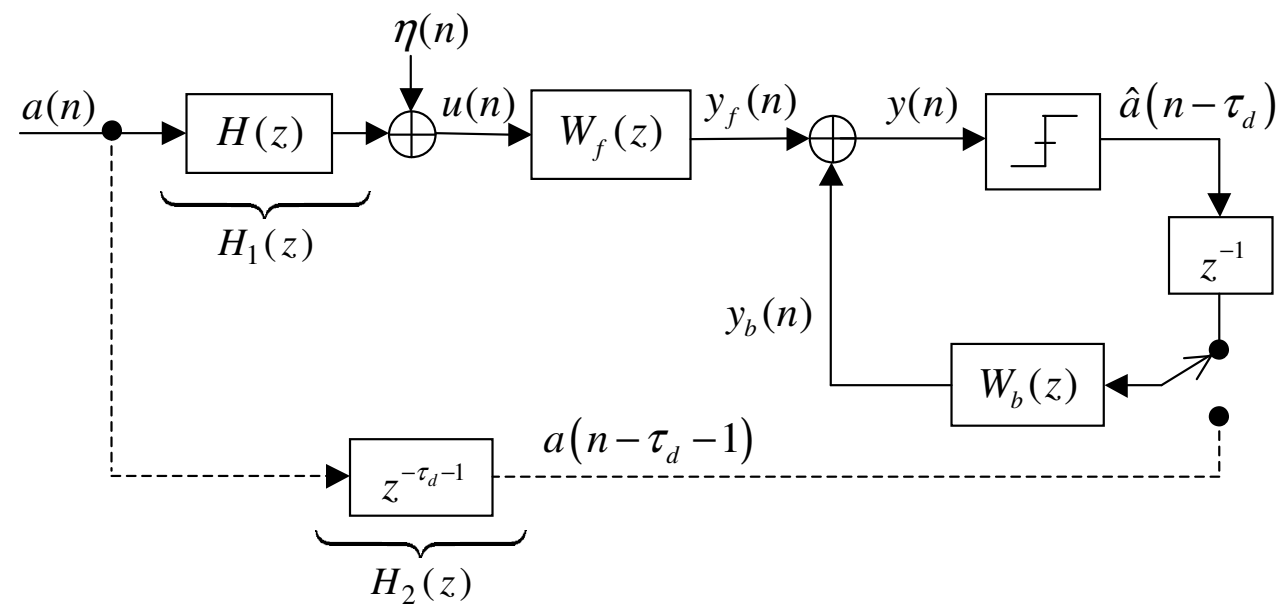

Figura 1.10: Modelo de um sistema de comunicação com DFE, assumindo-se decisões corretas.

Como $H_{2}(z)$ é um simples atraso, $H_{1}(z)$ e $H_{2}(z)$ não têm fator comum, exceto quando $H(z)$ for um atraso puro $z^{-1}$ [Papadias \& Paulraj, 1995]. Esse resultado possibilita interpretar o DFE como um receptor linear, similar a um equalizador sobre-amostrado, que pode ser descrito na ausência de ruído pelo seguinte sistema SIMO:

$$
\mathbf{u}_{f b}(n)=\mathcal{H}_{f b}^{T} \mathbf{a}(n)
$$

sendo

$$
\mathbf{a}(n)=[a(n) a(n-1) \cdots a(n-p)]^{T}
$$

com

$$
p=\left\{\begin{array}{lll}
N_{h}+M_{f}-2 & \text { se } & \tau_{d} \leq N_{h}+M_{f}-M_{b}-2 \\
\tau_{d}+M_{b} & \text { se } & \tau_{d} \geq N_{h}+M_{f}-M_{b}-2
\end{array}\right.
$$

e $\mathcal{H}_{f b}$ a matriz de convolução do canal equivalente. Se $\tau_{d} \leq N_{h}+M_{f}-M_{b}-2, \mathcal{H}_{f b}$ tem $M_{f}+M_{b}$ linhas e $N_{h}+M_{f}-1$ colunas e assume a forma

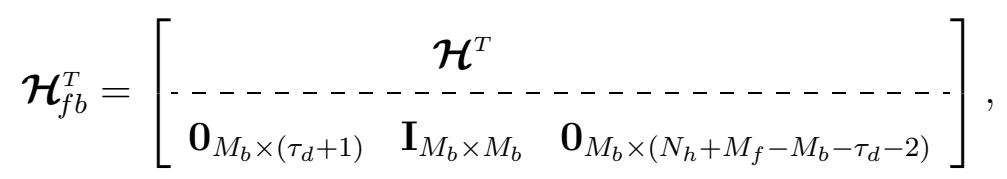

sendo $\mathcal{H}$ a matriz de convolução do canal $H(z)$. Em contrapartida, se $\tau_{d} \geq N_{h}+M_{f}-M_{b}-2$, $\mathcal{H}_{f b}$ tem $M_{f}+M_{b}$ linhas e $M_{b}+\tau_{d}+1$ colunas, ou seja,

$$
\boldsymbol{H}_{f b}^{T}=\left[\begin{array}{cc}
\boldsymbol{H}^{T} & \mathbf{0}_{M_{f} \times\left(M_{b}+\tau_{d}-N_{h}-M_{f}+2\right)} \\
\hdashline \mathbf{0}_{M_{b} \times\left(\tau_{d}+1\right)} & \mathbf{I}_{M_{b} \times M_{b}}
\end{array}\right] .
$$


O vetor de resposta combinada da cascata canal-equalizador pode ser expresso como

$$
\mathbf{s}_{f b}=\mathcal{H}_{f b} \mathbf{w}_{f b}
$$

sendo que $\mathbf{s}_{f b}$ tem dimensão $N_{h}+M_{f}-1$ se $\tau_{d} \leq N_{h}+M_{f}-M_{b}-2$ ou $M_{b}+\tau_{d}+1$ se $\tau_{d} \geq N_{h}+M_{f}-M_{b}-2$.

Assumindo decisões corretas e ausência de ruído, a equalização perfeita pode ser obtida, se as seguintes condições forem verificadas [Papadias \& Paulraj, 1995]:

$$
M_{b} \geq N_{h}-1 \quad \text { se } \quad \tau_{d} \leq N_{h}+M_{f}-M_{b}-2
$$

$\mathrm{Ou}$

$$
M_{f} \geq \tau_{d}+1 \quad \text { se } \quad \tau_{d} \geq N_{h}+M_{f}-M_{b}-2
$$

Neste caso, a matriz $\mathcal{H}_{f b}$ tem posto completo. Cabe observar que essas condições são suficientes, mas não necessárias.

Embora esse resultado tenha sido obtido a partir da consideração de decisões corretas e ausência de ruído, ele é interessante pois mostra que o DFE pode apresentar um bom desempenho na equalização de canais com resposta ao pulso unitário finita. Para exemplificar, considera-se o canal $H(z)=1+z^{-1}+4 z^{-2}+2 z^{-3}+4 z^{-4}+2 z^{-5}+z^{-6}+z^{-7}$ [Papadias \& Paulraj, 1995]. Nas figuras 1.11-a e 1.11-b, são mostrados respectivamente os módulos do operador de projeção para o LTE com $M=3$ e $M=10$, operando na taxa de símbolos. É possível observar que em ambos os casos, $\mathcal{P} \neq \mathbf{I}$. Se o modelo desse canal fosse obtido da sobre-amostragem com $L=2$, a equalização perfeita também não seria atingida, dado que os subcanais $C_{0}(z)=1+4 z^{-1}+4 z^{-2}+z^{-3}$ e $C_{1}(z)=1+2 z^{-1}+2 z^{-2}+z^{-3}$ possuem um zero em comum. Ao se considerar o DFE com decisões corretas, $M_{f}=3$ e $M_{b}=5$, obtém-se o operador de projeção da Figura 1.11-c. Neste caso, $\mathcal{P} \neq \mathbf{I}$ pois a configuração do DFE não atende (1.42). Considerando finalmente o DFE com $M_{f}=3$ e $M_{b}=7$, configuração que satisfaz (1.42), chega-se a $\mathcal{P}=\mathbf{I}$, como mostrado na Figura 1.11-d. Neste caso, mesmo sem sobre-amostragem, é possível passar de uma situação de submodelagem para a da ordem suficiente apenas com o uso do DFE, assumindo-se decisões corretas e ausência de ruído. 
a) $M=3$

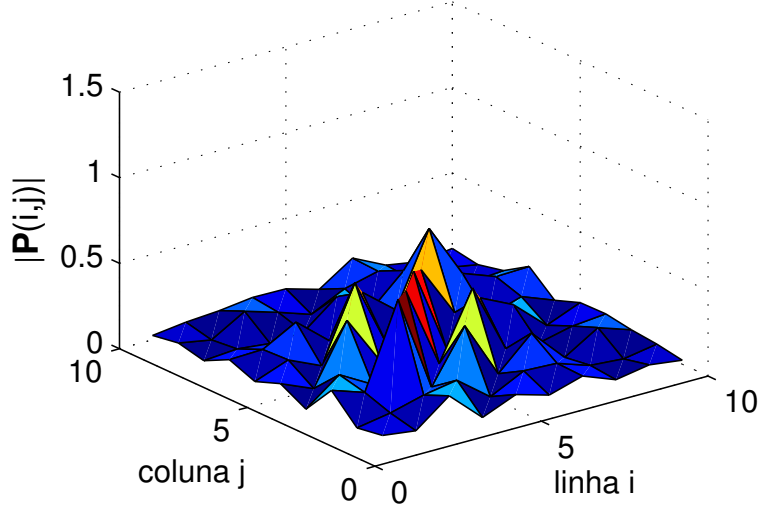

c) $M_{f}=3, M_{b}=5$

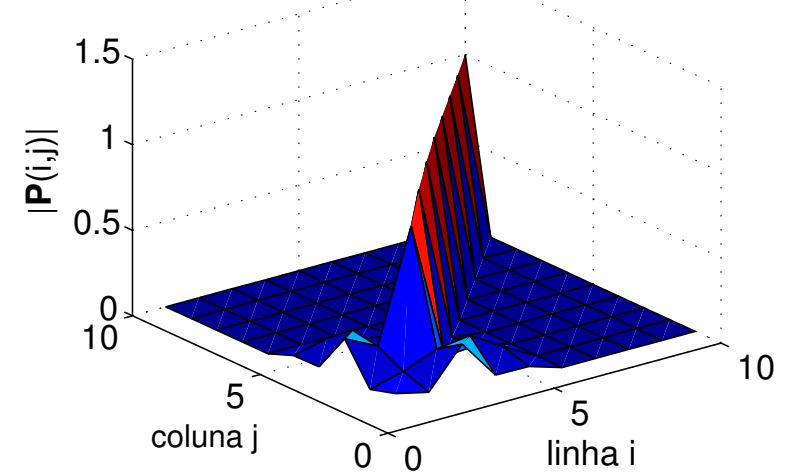

b) $M=10$

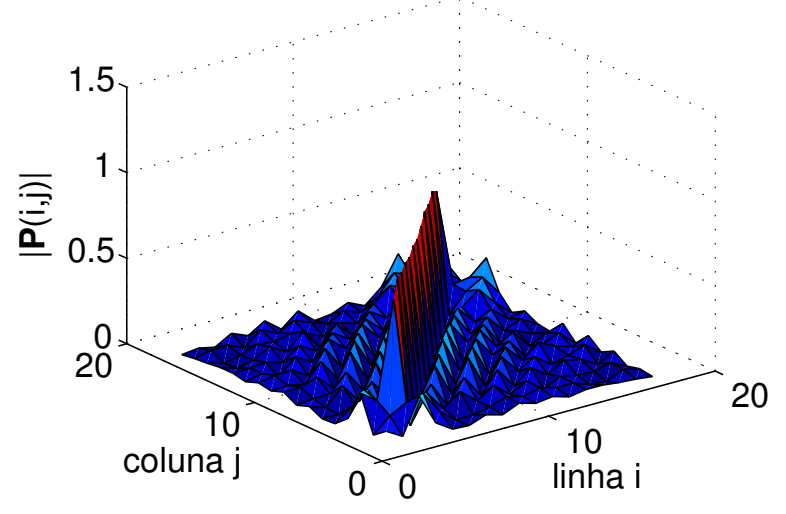

d) $M_{f}=3, M_{b}=7$

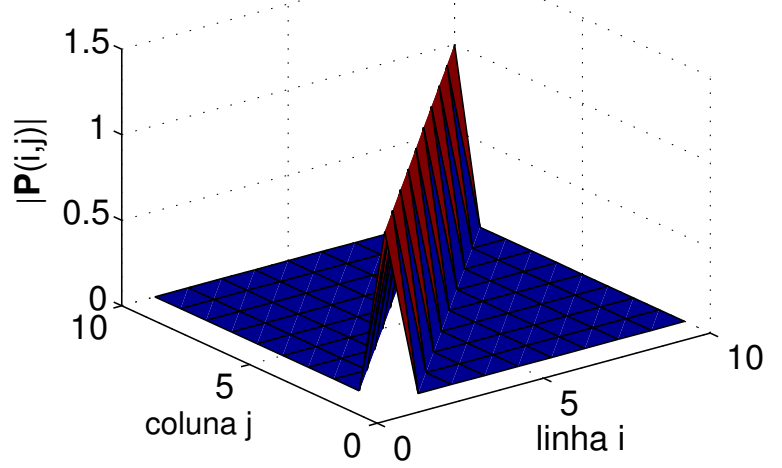

Figura 1.11: Módulo do operador de projeção $\mathcal{P}$ para o canal $H(z)=1+z^{-1}+4 z^{-2}+2 z^{-3}+$ $4 z^{-4}+2 z^{-5}+z^{-6}+z^{-7}$, sem sobre-amostragem; a) LTE $(M=3)$; b) LTE $(M=10)$; c) DFE $\left.\left(M_{f}=3, M_{b}=5\right) ; \mathrm{d}\right) \operatorname{DFE}\left(M_{f}=3, M_{b}=7\right)$.

\subsubsection{Outros equalizadores não-lineares}

Considerando um canal FIR invariante no tempo e o equalizador operando na taxa de símbolos (sem sobre-amostragem), Forney (1972) demonstrou que o equalizador ótimo é formado por um filtro casado com o canal de transmissão seguido do algoritmo de Viterbi [Viterbi, 1967; Forney, 1973; Ungerboeck, 1974]. Esse equalizador é não-linear e busca a seqüência de máxima verossimilhança. Segundo Forney (1972), essa solução apresenta excelentes resultados e é tão boa quanto às obtidas por estimadores ótimos de seqüências que utilizam outros critérios. No entanto, os maiores problemas desse esquema são a necessidade de se conhecer previamente o canal ou se se fazer uma boa estimativa [Magee \& Proakis, 1973] e a complexidade do algoritmo de Viterbi que cresce exponencialmente com a ordem 
do canal e com o número de símbolos do alfabeto da constelação considerada.

Comparando essa solução com o LTE, percebe-se uma limitação fundamental no equalizador linear. Dependendo do canal, ele poderá estar muito longe do ótimo segundo o critério da Máxima Verossimilhança (ML - Maximum Likelihood). Por isso, surgiram vários estudos na direção de se obter um equalizador intermediário entre o ótimo e o LTE. Diversas estruturas não-lineares têm sido consideradas, como as baseadas em séries de Volterra [Borys, 2001; Diniz, 1998] e as que utilizam redes neurais [Kechriotis et al., 1994a; Mulgrew, 1996; Diniz, 1998; Amari \& Cichocki, 1998; Destro, 1998; Bouchired et al., 1999; Silva, 2001]. As redes neurais, apesar de serem complexas, apresentam vantagens devido ao alto grau de paralelismo, o que as torna atraentes para implementação em circuitos integrados [Bouchired et al., 1999]. No entanto, a adaptação autodidata dessas redes quase sempre sofre com mínimos locais devido às não-linearidades da estrutura, somadas às não-linearidades do critério autodidata utilizado [Gomes \& Barroso, 1995]. Mesmo considerando a adaptação supervisionada, as redes neurais nem sempre apresentam vantagens em relação ao DFE, principalmente no que diz respeito ao esforço computacional dos algoritmos de treinamento [Silva, 2001]. Além disso, não se deve esquecer que o DFE possui uma estrutura não-linear simples e é bastante utilizado na prática [Ghosh, 1998]. Porém, um de seus maiores problemas é a propagação de erros devido à realimentação de decisões erradas [Casas, 1996; Fertner, 1998; Reuter et al., 2001].

Diante disso, o uso de uma estrutura híbrida formada pelo DFE e por uma rede neural pode ser uma solução interessante. Em [Silva, 2001; Silva \& Gerken, 2002a] foi proposto um equalizador híbrido supervisionado formado pelo DFE e uma rede neural recorrente. Na pior das hipóteses ele apresenta um desempenho igual ao das estruturas que o compõem, sendo melhor que cada uma delas em situações críticas [Silva \& Gerken, 2002a]. Nesse esquema, a rede neural é utilizada na malha de realimentação, a fim de evitar propagação de erros. Além disso, sua complexidade não é muito elevada, dado que a rede é constituída de apenas três neurônios. 


\subsection{Formulação do problema - caso MIMO}

Um diagrama de blocos da equalização multicanal (caso MIMO) é apresentado na Figura 1.3, página 6. As seqüências transmitidas $a_{i}(n), i=1,2, \ldots, N_{u}$ são assumidas i.i.d., nãogaussianas e independentes entre si. No receptor, há uma rede de antenas com $K\left(K>N_{u}\right)$ sensores que recebem misturas ruidosas dos sinais transmitidos, possuindo interferências do tipo co-canal (CCI - Co-Channel Interference) e intersimbólica (ISI - Intersymbol Interference). O sistema $\mathbf{H}$ é formado por $K N_{u}$ canais modelados por filtros FIR que ligam os usuários aos sensores. Supondo conhecido o número de usuários, o equalizador é formado por $N_{u}$ filtros adaptativos FIR com comprimento $M_{u}=K K_{t}$, sendo $K_{t}$ um número inteiro ligado à diversidade temporal. As saídas $y_{i}, i=1, \ldots, N_{u}$, são utilizadas para atualizar os coeficientes desses filtros por meio de um algoritmo autodidata. Na Figura 1.12, é mostrado um modelo desse sistema, considerando $N_{u}=2$ usuários e $K=3$ antenas. Os sinais $u_{1}(n)$, $u_{2}(n)$ e $u_{3}(n)$ representam as misturas capturadas pelos sensores e $\eta_{1}(n), \eta_{2}(n)$ e $\eta_{3}(n)$ são sinais de ruído considerado AWGN e com média nula.

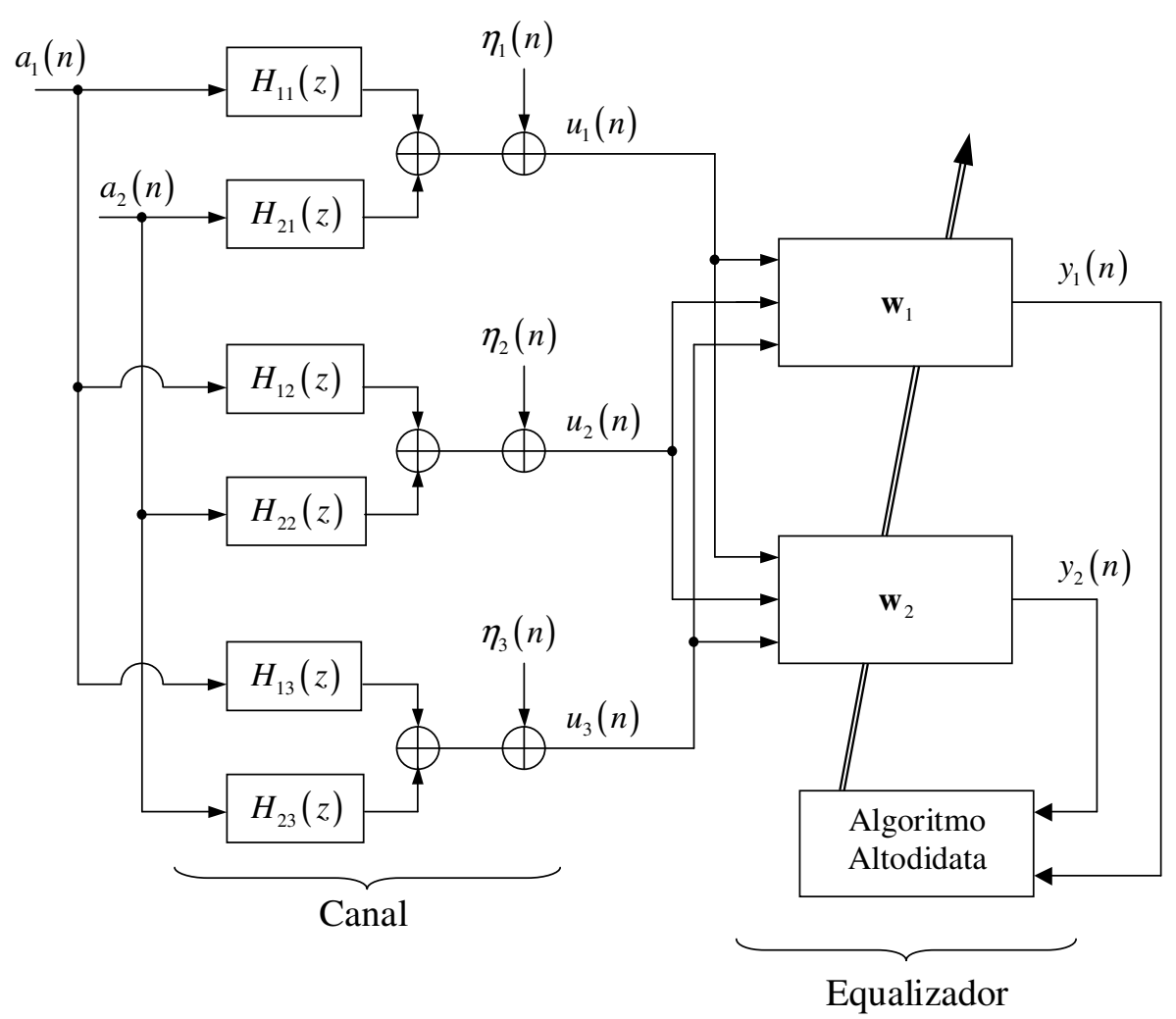

Figura 1.12: Modelo de um sistema de equalização espaço-temporal, considerando $N_{u}=2$ usuários e $K=3$ antenas. 
Assumindo diversidade temporal e espacial, o vetor de entrada de cada filtro do equalizador pode ser representado como

$$
\mathbf{u}^{T}(n)=\left[\begin{array}{llll}
\mathbf{u}_{1}^{T}(n) & \mathbf{u}_{2}^{T}(n) & \cdots & \mathbf{u}_{K}^{T}(n)
\end{array}\right]
$$

sendo

$$
\mathbf{u}_{p}(n)=\left[\begin{array}{llll}
u_{p}(n) & u_{p}(n-1) & \cdots & u_{p}\left(n-K_{t}+1\right)
\end{array}\right]^{T}
$$

$\operatorname{com} p=1,2, \ldots, K$.

Na ausência de ruído, o mapeamento entre a entrada e a saída do canal pode ser expresso como

$$
\mathbf{u}(n)=\underbrace{\left[\begin{array}{cccc}
\mathcal{H}_{11}^{T} & \mathcal{H}_{21}^{T} & \cdots & \mathcal{H}_{N_{u} 1}^{T} \\
\mathcal{H}_{12}^{T} & \mathcal{H}_{22}^{T} & \cdots & \mathcal{H}_{N_{u} 2}^{T} \\
\vdots & \vdots & \ddots & \vdots \\
\mathcal{H}_{1 K}^{T} & \mathcal{H}_{2 K}^{T} & \cdots & \mathcal{H}_{N_{u} K}^{T}
\end{array}\right]}_{\mathcal{H}^{T}} \underbrace{\left[\begin{array}{c}
\mathbf{a}_{1}(n) \\
\mathbf{a}_{2}(n) \\
\vdots \\
\mathbf{a}_{N_{u}}(n)
\end{array}\right]}_{\mathbf{a}(n)}
$$

sendo $\mathbf{a}_{p}(n)=\left[\begin{array}{llll}a_{p}(n) & a_{p}(n-1) & \ldots & a_{p}\left(n-K_{t}-K_{c}+2\right)\end{array}\right]^{T}, K_{c}-1$ a ordem de cada canal e $\mathcal{H}_{i j}$ a matriz de convolução do canal $H_{i j}(z)$, que liga o usuário $i$ à antena $j$.

Os sinais de saída dos filtros do equalizador podem ser representados como

$$
y_{i}(n)=\mathbf{w}_{i}^{T} \mathbf{u}(n)
$$

ou ainda

$$
y_{i}(n)=\mathbf{s}_{i}^{T} \mathbf{a}(n)
$$

sendo que $\mathbf{s}_{i}(n)=\mathcal{H} \mathbf{w}_{i}(n), \quad i=1,2 \ldots, N_{u}$ representa a resposta combinada do usuário $i$.

Como no caso monousuário, uma condição suficiente, mas não necessária para a equalização perfeita é $\mathcal{P}=\mathbf{I}$. Para isso, as seguintes condições devem ser satisfeitas [Papadias \& Paulraj, 1997a]:

C1. o número de coeficientes de cada filtro do equalizador deve atender à seguinte relação

$$
M_{u} \geq \frac{N_{u}\left(K_{c}-1\right)}{1-N_{u} / K}
$$


C2. os canais virtuais $H_{i}(z), i=1,2, \ldots, N_{u}$, definidos como

$$
H_{i}(z)=\sum_{j=1}^{N_{u}} H_{j i}\left(z^{N_{u}}\right) z^{j-1},
$$

não devem ter zeros em comum.

A condição C1 é obtida fazendo com que o número de colunas da matriz de convolução do canal $\mathcal{H}$ seja maior ou igual ao número de linhas. Além de $\mathrm{C} 1$, deve-se impor $\mathrm{C} 2$ para garantir que o número de colunas linearmente independentes de $\mathcal{H}$ seja igual a $N_{u}\left(K_{t}+K_{c}-1\right)$. Para que o denominador de (1.49) seja positivo, deve-se ter $N_{u} / K<1$, isto é, o número de sensores deve ser maior que o número de usuários. Além disso, pode-se verificar que o número de coeficientes de cada filtro do equalizador deve aumentar com o número de fontes e com o comprimento da resposta ao pulso unitário de cada canal. Embora a sobre-amostragem não seja necessária para garantir a equalização perfeita, ela pode ser utilizada para melhorar o condicionamento da matriz de autocorrelação.

\subsection{Métodos para projeto de algoritmos adaptativos}

Em geral, os algoritmos utilizados para adaptação dos coeficientes do LTE ou DFE ${ }^{1}$ podem ser descritos pela seguinte equação [Benveniste et al., 1987]

$$
\mathbf{w}(n)=\mathbf{w}(n-1)+\rho(n) \widehat{\mathbf{g}}(\mathbf{w}(n-1), \mathbf{u}(n))+\rho^{2}(n) v(\mathbf{w}(n-1), \mathbf{u}(n)),
$$

sendo $\mathbf{w}(n)$ o vetor de coeficientes que deve ser atualizado recursivamente, $\mathbf{u}(n)$ o vetor de entrada que representa as observações do sistema, $\rho(n)$ o passo de adaptação, $v(\mathbf{w}, \mathbf{u})$ uma pequena perturbação no algoritmo (usualmente $v=0$ ) e $\widehat{\mathbf{g}}(\mathbf{w}, \mathbf{u})$ a função que essencialmente define como o parâmetro w é atualizado em função das novas observações.

Segundo [Benveniste et al., 1987, p. 55-56], o projeto de um algoritmo adaptativo da forma (1.50) pode ser feito em quatro etapas enumeradas a seguir .

\section{Escolha de uma função custo $J(\mathbf{w}, \mathbf{u})$ a ser minimizada.}

Usualmente, a função custo é expressa pela esperança matemática $\mathrm{E}\{\cdot\}$ de uma função que depende do vetor de estados $\mathbf{u}(n)$ formado pelas novas observações e do vetor de coeficientes $\mathbf{w}$.

\footnotetext{
${ }^{1}$ Aqui se considerou o caso monousuário, mas sua extensão para o caso multiusuário é direta.
} 


\section{Escolha do método para minimizar $J(\mathbf{w}, \mathbf{u})$.}

O método do gradiente e o de Newton são os mais utilizados na prática. No método do gradiente, $\mathbf{g}(\mathbf{w}, \mathbf{u})$ é o vetor gradiente de $J(\mathbf{w}, \mathbf{u})$. No método de Newton, além da obtenção do gradiente, a matriz hessiana precisa ser invertida.

\section{Obtenção do algoritmo exato.}

Nesta etapa, obtêm-se algoritmos da forma

$$
\mathbf{w}(n)=\mathbf{w}(n-1)+\rho(n) \mathbf{g}(\mathbf{w}(n-1), \mathbf{u}(n)),
$$

sendo $\mathbf{g}(\mathbf{w}(n-1), \mathbf{u}(n))$ o vetor gradiente exato ou a inversa da matriz hessiana vezes o vetor gradiente, dependendo do método utilizado para minimização da função custo. Esses algoritmos não são muito utilizados na prática devido à dificuldade de obter o gradiente e/ou a hessiana de forma exata.

\section{Desenvolvimento e análise subseqüente do algoritmo aproximado.}

Na maioria das vezes, os algoritmos obtidos na Etapa 3 são considerados muito complexos. Desta forma, eles devem ser simplificados ou reajustados através de aproximações heurísticas. Os algoritmos aproximados devem ser testados em simulações, e se possível, analisados segundo um método estatístico.

Dependendo da aplicação, algumas etapas desse método podem ser eliminadas [Benveniste et al., 1987]. A Etapa 4 é essencial, quando se deseja obter algoritmos para aplicações em tempo real, como é o caso da equalização autodidata.

Neste trabalho, as palavras "determinístico" e "estocástico" são utilizadas para designar o tipo de estimativa do gradiente. Assim, algoritmos da forma (1.51) que utilizam o gradiente exato são chamados de algoritmos do gradiente determinístico. Algoritmos aproximados que utilizam estimativas instantâneas do gradiente são denominados de algoritmos do gradiente estocástico. Conseqüentemente, algoritmos baseados nos métodos de Newton e de quaseNewton são considerados respectivamente como do gradiente determinístico e do gradiente estocástico. Na prática, nem sempre é possível obter o gradiente de forma exata. Assim, nas simulações com os algoritmos do gradiente determinístico, são utilizadas estimativas em bloco, sendo que quanto maior o número de amostras do bloco de dados, mais precisas serão as estimativas. 
Neste contexto, uma outra forma de obter diretamente algoritmos aproximados de tempo discreto é a discretização de algoritmos de tempo contínuo. As características do algoritmo resultante dependerão do método de discretização. Ao se considerar, por exemplo, o algoritmo do gradiente de controle adaptativo [Aström \& Wittenmark, 1995], pode-se chegar aos algoritmos LMS e NLMS, utilizando-se respectivamente os métodos de Euler progressivo e regressivo [Gerken et al., 2000; Jojoa, 1999, 2003]. É importante salientar que algumas aproximações devem ser feitas depois da discretização, a fim de garantir a estabilidade numérica dos algoritmos resultantes.

Cabe observar ainda que em [Papadias \& Slock, 1997] foi proposta uma metodologia para a obtenção de algoritmos de equalização autodidata a partir de algoritmos clássicos de filtragem adaptativa. Segundo Papadias e Slock, algoritmos autodidatas com equação de atualização da forma do algoritmo do Módulo Constante podem ser obtidos através do princípio da separação. Esse princípio estabelece que para se chegar ao algoritmo autodidata, deve-se substituir o sinal desejado do algoritmo supervisionado por uma função não-linear, que depende da saída do equalizador e das estatísticas do sinal transmitido. Desta forma, o algoritmo do Módulo Constante pode ser obtido do algoritmo LMS, substituindo-se $d(n)$ do $\operatorname{erro} e(n)=y(n)-d(n)$ por $\hat{d}(n) \approx \varphi(y(n))=\left(R_{2}^{a}-|y(n)|^{2}+1\right) y(n)$.

\subsection{Objetivos e justificativa}

Nesta tese, são abordados métodos de desconvolução autodidata baseados em estatísticas de ordem superior a 2 (HOS), aplicados à equalização de canais de comunicação tanto no caso monousuário como no caso multiusuário.

Dentre os algoritmos de equalização autodidata que utilizam HOS, destacam-se o algoritmo do Módulo Constante (CMA - Constant Modulus Algorithm) proposto por Godard (1980) e o algoritmo Super-Exponencial proposto por Shalvi e Weinstein (1990). O primeiro é obtido a partir da função custo de Godard e o segundo através de um procedimento iterativo, cuja função custo pertence à família de funções objetivo de Donoho [Donoho, 1981]. Além desses, podem ser citados outros algoritmos que foram propostos recentemente, como por exemplo, o GCMA (Generalized Constant Modulus Algorithm) [Cavalcanti et al., 1998], o AC-CMA (Accelerated Constant Modulus Algorithm) [Silva et al., 2002b, 2004d] e 
o BQNA (Blind Quasi-Newton Algorithm) [Licciardi, 2003]. A natureza não-linear, a multimodalidade e a dependência da qualidade das estimativas desses algoritmos tornam uma análise de convergência e estabilidade consistente consideravelmente difícil, e este ainda é um problema em aberto.

Em [Miranda, 1999], foi feito um estudo da relação dos critérios do Módulo Constante e de Shalvi-Weinstein. Nesse trabalho, foram abordadas as funções custo e as equações de atualização dos coeficientes. Verificou-se que a velocidade de convergência e a qualidade das estimativas dos coeficientes do equalizador estão relacionadas com o fator de ponderação do gradiente estimado e com os autovalores da matriz de autocorrelação. A presença da inversa dessa matriz no algoritmo de Shalvi-Weinstein garante uma velocidade ótima de convergência, às custas de uma elevada complexidade computacional. Um problema presente nesses algoritmos é a existência de pontos de equilíbrio indesejáveis na função custo. Além disso, uma questão ainda em aberto é a faixa do passo de adaptação que assegura a convergência do algoritmo do Módulo Constante. Ainda nesse trabalho, foram apresentadas duas formas de acelerar a convergência dos algoritmos. Uma baseada no uso do passo de adaptação ótimo para a velocidade de convergência, levando a algoritmos do tipo ShalviWeinstein e outra baseada na multiplicação do vetor gradiente pela inversa da matriz hessiana (método de Newton). Neste caso, a inicialização do algoritmo é um fator fundamental. Dependendo das condições iniciais, a inversa da matriz hessiana deixa de ser positiva-definida, o que resulta numa convergência em potencial para pontos de mínimo local da função custo. Desta forma, o desempenho dos algoritmos autodidatas depende da escolha correta do passo de adaptação, das condições iniciais e da qualidade das estimativas.

O CMA é um dos algoritmos mais utilizados na prática pois possui baixa complexidade computacional [Ding \& Li, 2001; Licciardi, 2003]. Devido à natureza não-linear e à multimodalidade de sua função custo, ele pode apresentar problemas de convergência para mínimos indesejáveis e sofre forte influência das condições iniciais. Um estudo das condições em que esse algoritmo apresenta um desempenho satisfatório é algo de interesse e ainda é um problema em aberto. A possibilidade de se obter um algoritmo com maior velocidade de convergência também é importante para a aplicação em questão. Além disso, com a utilização do DFE e/ou outros sistemas não-lineares adaptados de forma autodidata, pode- 
se conseguir potencialmente um melhor desempenho na equalização de canais esparsos ou não-lineares. Uma extensão desse estudo para o caso multiusuário torna o trabalho mais próximo dos sistemas atuais de comunicação digital, em que se considera o múltiplo acesso.

\subsection{Contribuições}

As principais contribuições deste trabalho são enumeradas a seguir.

\section{Análise de convergência do SD-CMA.}

Inspirando-se na análise de convergência do algoritmo Super-Exponencial no domínio da resposta combinada, foi feita uma análise de convergência do SD-CMA (Steepest Descent Constant Modulus Algorithm). A partir dessa análise, obteve-se o intervalo do passo de adaptação que assegura sua convergência.

\section{Análise de tracking de algoritmos autodidatas no caso monousuário.}

Baseando-se na relação de conservação de energia de [Sayed, 2003], foi feita uma análise de tracking unificada de algoritmos autodidatas que satisfazem tal relação. Seguindo os mesmos passos de [Yousef \& Sayed, 1999; Mai \& Sayed, 2000], obtiveram-se resultados mais abrangentes. Verificou-se que a razão entre os valores mínimos do MSE (MeanSquare-Error) dos algoritmos CMA e SWA (Shalvi-Weinstein Algorithm) é igual à razão obtida em [Eweda, 1994] para os algoritmos LMS (Least-Mean-Square) e RLS (Recursive Least-Squares).

\section{Algoritmo do Módulo Constante Acelerado.}

Tendo como motivação o bom desempenho do AAFA (Accelerating Adaptive Filtering Algorithm) apresentado em [Gerken et al., 2000; Jojoa et al., 2001b; Jojoa, 2003] e obtido a partir da discretização do algoritmo acelerador de tempo contínuo de [Pait, 1998], foi proposto um novo algoritmo para equalização autodidata denominado ACCMA (Accelerated Constant Modulus Algorithm). Às custas de um moderado aumento da complexidade computacional, ele apresenta uma boa capacidade de tracking e uma melhor habilidade para evitar mínimos locais suaves que o CMA. Esse algoritmo também foi estendido para o caso multiusuário. 


\section{Extensão do Algoritmo de Shalvi-Weinstein para o caso multiusuário.}

O SWA no caso monousuário pode ser interpretado como o CMA com passo de adaptação ótimo para velocidade de convergência [Mboup \& Regalia, 2000; Miranda, 1999]. Utilizando esse resultado foi feita uma extensão desse algoritmo para o caso multiusuário.

\section{Extensão da análise de tracking para o caso multiusuário.}

Levando em conta as considerações a respeito do termo que penaliza as correlações cruzadas entre os usuários, a análise de tracking feita no caso SISO foi estendida para o caso MIMO.

\section{Algoritmo autodidata concorrente para adaptação do DFE.}

Baseando-se no algoritmo concorrente de [Chen, 2003] e no algoritmo autodidata que evita soluções degeneradas de [Szczecinski \& Gei, 2002], foi proposto um algoritmo concorrente para adaptação autodidata do DFE. Esse algoritmo apresenta um melhor desempenho que o de [Szczecinski \& Gei, 2002].

\section{Equalizador híbrido autodidata.}

A adaptação autodidata do equalizador híbrido, formado por um DFE e uma rede neural recorrente (RNN - Recurrent Neural Network) de [Silva, 2001; Silva \& Gerken, 2002a] foi considerada. O equalizador resultante apresenta um bom desempenho quando comparado com o DFE ou LTE autodidatas.

Como resultado dessas contribuições, foram publicados os seguintes artigos:

- SILVA, M. T. M.; MIRANDA, M. D. Tracking issues of some blind equalization algorithms. IEEE Signal Processing Letters, v. 11, p. 760-763, Sept. 2004.

- SILVA, M. T. M.; GERKEN, M.; MIRANDA, M. D. A Blind Hybrid Decision Feedback Equalizer. In: SIMPÓSIO BRASILEIRO DE REDES NEURAIS, 8., 2004, São Luís. Anais. 1 CD-ROM.

- SILVA, M. T. M.; MIRANDA, M.; SOARES, R. Concurrent Blind Decision Feedback Equalizer. In: INTERNATIONAL WORKSHOP ON TELECOMMUNICATIONS, 2004, Santa Rita do Sapucaí. Proceedings of IWT'2004, p. 107-112. 
- SILVA, M. T. M.; GERKEN, M.; MIRANDA, M. D. An accelerated constant modulus algorithm for space-time blind equalization. In: EUROPEAN SIGNAL PROCESSING CONFERENCE, 12., 2004, Vienna. Proceedings of EUSIPCO'2004. p. 1853-1856.

- SILVA, M. T. M.; MIRANDA, M. D.; LICCIARDI JR., A. N. A robust algorithm for blind space-time equalization. In: IEEE INTERNATIONAL CONFERENCE ON ACOUSTICS, SPEECH AND SIGNAL PROCESSING, 2004, Montreal. Proceedings of ICASSP'2004. v. IV, p. 857-860.

- SILVA, M. T. M.; GERKEN, M.; MIRANDA, M. D. Um algoritmo do Módulo Constante Acelerado para equalização espaço-temporal. In: SIMPÓSIO BRASILEIRO DE TELECOMUNICAÇÕES, 20., 2003, Rio de Janeiro. Anais. 1 CD-ROM.

- SILVA, M. T. M.; GERKEN, M. A RNN-LC Hybrid Equalizer. In: EUROPEAN SIGNAL PROCESSING CONFERENCE, 11., 2002, Toulouse. Proceedings of EUSIPCO'2002. p. 341-344.

- SILVA, M. T. M.; GERKEN, M.; MIRANDA, M. D. An Accelerated Constant Modulus Algorithm. In: INTERNATIONAL TELECOMMUNICATIONS SYMPOSIUM, 2002, Natal. Proceedings of ITS'2002. 1 CD-ROM.

\subsection{Organização da tese}

Baseando-se nos métodos para projeto de filtros adaptativos apresentados na Seção 1.5, a tese foi estruturada em 6 capítulos.

No Capítulo 2, as principais funções custo das técnicas que utilizam HOS são apresentadas e analisadas. A partir dessas funções, algoritmos do gradiente determinístico para o caso monousuário são obtidos. Inspirando-se na análise de convergência do algoritmo SuperExponencial no domínio da resposta combinada, foi feita uma análise para o SD-CMA, obtendo-se o intervalo do passo de adaptação que assegura sua convergência.

No Capítulo 3, a partir dos algoritmos apresentados no Capítulo 2, são obtidos algoritmos do gradiente estocástico para equalização monocanal. Um novo algoritmo autodidata 
é proposto a partir da discretização de um algoritmo de tempo contínuo de controle adaptativo. A relação de conservação de energia de [Sayed, 2003] é revisitada, o que levou a uma análise de tracking unificada dos algoritmos autodidatas que satisfazem tal relação. O algoritmo concorrente autodidata de [Chen, 2003] também é revisitado e o capítulo termina com resultados de simulações.

No Capítulo 4, considera-se a equalização espaço-temporal. Os algoritmos do gradiente estocástico e a análise de tracking do Capítulo 3 são estendidos para o caso multiusuário. Resultados de simulações são apresentados a fim de comparar os algoritmos e validar os resultados da análise.

No Capítulo 5, um algoritmo concorrente para adaptação autodidata do DFE é proposto. Uma adaptação autodidata para o equalizador híbrido de [Silva, 2001; Silva \& Gerken, 2002a] também é considerada. A fim de comparar essas técnicas são apresentados alguns resultados de simulações.

Finalmente, no Capítulo 6, são apresentadas as conclusões do trabalho e as propostas para trabalhos futuros. 


\section{Capítulo 2}

\section{Algoritmos determinísticos para equalização monocanal}

Neste capítulo, são abordados os algoritmos do gradiente determinístico decorrentes de critérios clássicos de equalização autodidata. Inicialmente, são apresentadas a definição e as relações de cumulantes das seqüências envolvidas no processo de equalização. A equivalência entre as funções custo do Módulo Constante (Godard) e de Shalvi-Weinstein é revisitada. São obtidos seus gradientes, pontos estacionários e algoritmos determinísticos. A utilização das ferramentas de filtragem adaptativa supervisionada na análise de convergência e estabilidade desses algoritmos nem sempre é direta e muitas vezes não é aplicável. Isso ocorre devido à natureza não-linear e à multimodalidade dos critérios autodidatas. Em contrapartida, a literatura contém alguns resultados da análise do problema no domínio da resposta combinada da cascata canal-equalizador e que podem ser levados para o domínio dos coeficientes do equalizador. Desta forma, é feita uma análise de convergência de algoritmos do tipo SuperExponencial no domínio da resposta combinada. Inspirando-se nesse resultado, é feita uma análise do algoritmo do Módulo Constante baseado no gradiente determinístico. Em especial, obtém-se o intervalo do passo de adaptação que assegura sua convergência. O capítulo termina com resultados de simulações e conclusões.

Ao longo deste capítulo as seguintes considerações são válidas:

- para compactar a notação, consideram-se

$$
\mathbf{s}(n)=\mathbf{s}_{n}, \quad \mathbf{w}(n)=\mathbf{w}_{n}, \quad y(n)=y_{n}, \quad \mathbf{u}(n)=\mathbf{u}_{n} \quad \text { e } \quad f\left(\mathbf{s}_{n}\right)=f_{n}
$$


- dado um vetor real de resposta combinada $\mathbf{s}_{n}$, sua $m$-ésima potência de Hadamard é definida como

$$
\mathbf{s}_{n}^{\odot m}=\underbrace{\mathbf{s}_{n} \odot \cdots \odot \mathbf{s}_{n}}_{m \text { vetores }}=\left[\begin{array}{llll}
s_{n, 0}^{m} & s_{n, 1}^{m} & \cdots & s_{n, N_{h}+M-1}^{m}
\end{array}\right]^{T},
$$

sendo que $\odot$ representa a operação de multiplicação elemento a elemento de vetores e $s_{n, k}$ o $k$-ésimo elemento de $\mathbf{s}_{n}$. No caso complexo, expressões da forma $\mathbf{s}^{\odot p} \odot\left(\mathbf{s}^{*}\right)^{\odot(p-1)}$ são freqüentemente encontradas. Assim, para compactar a notação considera-se

$$
\mathbf{S}^{\odot<2 p-1>}=\mathbf{s}^{\odot p} \odot\left(\mathbf{s}^{*}\right)^{\odot(p-1)},
$$

sendo seu $k$-ésimo componente igual a

$$
\left[\mathbf{s}^{\odot<2 p-1>}\right]_{k}=\left|s_{k}\right|^{2 p-2} s_{k}
$$

- como medida de desempenho, utiliza-se a interferência intersimbólica (ISI) residual definida como

$$
\operatorname{ISI}(\mathbf{s})=\frac{\sum_{k}\left|s_{k}\right|^{2}}{\left|s_{k_{m}}\right|^{2}}-1,
$$

sendo $s_{k_{m}}$ o componente de maior valor absoluto do vetor de resposta combinada $\mathbf{s}$ [Shalvi \& Weinstein, 1994];

- para a maioria das constelações complexas usadas em comunicações digitais, vale a propriedade de simetria circular [Shalvi \& Weinstein, 1994; Proakis, 1996], ou seja,

$$
\mathrm{E}\left\{a^{2}\right\}=\mathrm{E}\left\{\left(a^{*}\right)^{2}\right\}=0
$$

- assume-se que $u(n)$, sinal de entrada do equalizador, é estacionário até ordem quatro [Regalia, 1999].

\subsection{Cumulantes: definição e relações importantes}

Uma seqüência de variáveis aleatórias $x_{1}, x_{2}, \ldots, x_{m}$ é completamente descrita através da função densidade de probabilidade (f.d.p.) conjunta denotada por [Picinbono, 1993]

$$
f_{\mathbf{x}}(\mathbf{x})=f_{x_{1}, x_{2}, \ldots, x_{m}}\left(x_{1}, x_{2}, \ldots, x_{m}\right)
$$


Agrupando as variáveis aleatórias no vetor

$$
\mathbf{x}=\left[\begin{array}{llll}
x_{1} & x_{2} & \cdots & x_{m}
\end{array}\right]^{T}
$$

e definindo o vetor de parâmetros

$$
\boldsymbol{\omega}=\left[\begin{array}{llll}
\omega_{1} \omega_{2} & \cdots & \omega_{m}
\end{array}\right]^{T}
$$

a primeira função característica de $\mathbf{x}$ pode ser expressa como

$$
\phi_{\mathbf{x}}(\boldsymbol{\omega})=\int_{-\infty}^{\infty} \cdots \int_{-\infty}^{\infty} f_{\mathbf{x}}(\mathbf{x}) e^{j \boldsymbol{\omega}^{T} \mathbf{x}} d x_{1} \cdots d x_{m}
$$

ou ainda utilizando o operador esperança

$$
\phi_{\mathbf{x}}(\boldsymbol{\omega}) \triangleq \mathrm{E}\left\{\exp \left(j \boldsymbol{\omega}^{T} \mathbf{x}\right)\right\}=\mathrm{E}\left\{\exp \left(j \sum_{k=1}^{m} \omega_{k} x_{k}\right)\right\}
$$

De (2.5) pode-se interpretar a primeira função característica $\phi_{\mathbf{x}}(\boldsymbol{\omega})$ como a transformada de Fourier (com os sinais de $\omega_{1}, \omega_{2}, \ldots, \omega_{m}$ trocados) da f.d.p. conjunta $f_{\mathbf{x}}(\mathbf{x})$. Desta forma, tanto a f.d.p. $f_{\mathbf{x}}(\mathbf{x})$ quanto a primeira função característica $\phi_{\mathbf{x}}(\boldsymbol{\omega})$ possuem a mesma informação sobre a seqüência de variáveis aleatórias $x_{1}, x_{2}, \cdots, x_{m}$ [Picinbono, 1993]. A primeira função característica também é conhecida como geradora de momentos. De fato, os momentos são os coeficientes da expansão em série de McLaurin de $\phi_{\mathbf{x}}(\boldsymbol{\omega})$. Desta forma, o momento conjunto de ordem $n$ é definido como

$$
\operatorname{mom}\left(x_{1}, x_{2}, \ldots, x_{m}\right) \triangleq \mathrm{E}\left\{\prod_{k=1}^{m} x_{k}^{q_{k}}\right\}
$$

sendo $q_{k}$ inteiros positivos tal que $\sum_{k=1}^{m} q_{k}=n$. Esse momento pode ser obtido a partir da derivada da primeira função característica, ou seja,

$$
\operatorname{mom}\left(x_{1}, x_{2}, \ldots, x_{m}\right)=\left.(-j)^{n} \frac{\partial^{n} \phi_{\mathbf{x}}(\boldsymbol{\omega})}{\partial \omega_{1}^{q_{1}} \partial \omega_{2}^{q_{2}} \cdots \partial \omega_{m}^{q_{m}}}\right|_{\boldsymbol{\omega}=\mathbf{0}}
$$

Considerando sem perda de generalidade $m=n$, então $q_{k}=1$ para todo $k$ e (2.7) se reduz a

$$
\operatorname{mom}\left(x_{1}, x_{2}, \ldots, x_{m}\right)=\left.(-j)^{n} \frac{\partial^{n} \phi_{\mathbf{x}}(\boldsymbol{\omega})}{\partial \omega_{1} \partial \omega_{2} \cdots \partial \omega_{m}}\right|_{\boldsymbol{\omega}=\mathbf{0}}
$$

Pode-se demonstrar que (2.8) é equivalente a (2.7). Para isso, caso algum $q_{k}>1$, basta utilizar um conjunto de variáveis aleatórias aumentado dado por $\left\{\tilde{x}_{k}\right\}_{k=1}^{n}$, sendo algumas variáveis $\tilde{x}_{k}$ idênticas entre si, de acordo com os valores de $q_{k}$ [Gardner \& Spooner, 1994]. 
A segunda função característica de $\mathbf{x}$ é o logaritmo natural de $\phi_{\mathbf{x}}(\boldsymbol{\omega})$, ou seja,

$$
\psi_{\mathbf{x}}(\boldsymbol{\omega})=\ln \phi_{\mathbf{x}}(\boldsymbol{\omega})
$$

Essa função possui propriedades interessantes e uma forma particularmente simples para algumas distribuições [Picinbono, 1993]. Os coeficientes da expansão em série de McLaurin de $\psi_{\mathbf{x}}(\boldsymbol{\omega})$ são chamados de cumulantes. Desta forma, o cumulante conjunto de ordem $n$ é definido a partir da derivada da segunda função característica, ou seja,

$$
\left.\operatorname{cum}\left(x_{1}, x_{2}, \ldots, x_{m}\right) \triangleq(-j)^{n} \frac{\partial^{n} \psi_{\mathbf{x}}(\boldsymbol{\omega})}{\partial \omega_{1} \partial \omega_{2} \cdots \partial \omega_{m}}\right|_{\boldsymbol{\omega}=\mathbf{0}} .
$$

De forma análoga à (2.8), essa definição também pode ser considerada geral [Gardner \& Spooner, 1994].

Os cumulantes têm um papel importante em processamento de sinais por possuírem as seguintes propriedades [Mendel, 1991; Picinbono, 1993]:

P1) Utilizando as propriedades de derivada parcial e a definição de função característica, verifica-se que os cumulantes satisfazem a propriedade de linearidade, ou seja, se $b_{k}$, $k=1, \ldots, m$, são constantes, então

$$
\operatorname{cum}\left(\sum_{k=1}^{m} b_{k} x_{k}\right)=\sum_{k=1}^{m} b_{k} \operatorname{cum}\left(x_{k}\right)
$$

P2) Se $x_{1}, x_{2}, \ldots, x_{m}$ puderem ser divididos em dois ou mais subconjuntos estatisticamente independentes, então o cumulante conjunto é nulo.

P3) Se $x_{1}, x_{2}, \ldots, x_{m}$ são conjuntamente gaussianos, então o cumulante conjunto de ordem superior a 2 é nulo.

P4) Se as variáveis aleatórias $x_{1}, x_{2} \ldots, x_{m}$ são independentes das variáveis aleatórias $y_{1}, y_{2} \ldots, y_{m}$, então

$$
\operatorname{cum}\left(x_{1}+y_{1}, \cdots, x_{m}+y_{m}\right)=\operatorname{cum}\left(x_{1}, \cdots, x_{m}\right)+\operatorname{cum}\left(y_{1}, \cdots, y_{m}\right)
$$

As propriedades $\mathrm{P} 1$ e P4 são as principais razões da preferência do uso de cumulantes em relação aos momentos em alguns problemas de estimação. Enquanto os cumulantes são cumulativos, os momentos não são, o que justifica o nome "cumulante" [Gardner \& Spooner, 
1994]. Além disso, através de P3 verifica-se que cumulantes de ordem superior a dois têm uma tolerância natural a ruído gaussiano. Desta forma, eles constituem a principal ferramenta no processamento de sinais não-gaussianos. O papel que eles desempenham em processos não-gaussianos é similar ao das covariâncias no caso gaussiano: sob certas considerações eles podem ser estimados de forma consistente e contêm informação sobre os parâmetros do processo [Porat, 1994].

No caso de interesse, os cumulantes mais utilizados são os de segunda e quarta ordens. Cumulantes de ordem três de processos com distribuição simétrica são nulos. Além disso, em alguns processos aleatórios, o cumulante de ordem três é extremamente pequeno em relação ao de ordem quatro, o que justifica a utilização mais freqüente do último em relação ao primeiro [Mendel, 1991]. Se as variáveis aleatórias $x_{1}, x_{2}, \ldots, x_{M}$ tiverem média nula, o cumulante e o momento de ordem dois coincidem, ou seja,

$$
\operatorname{cum}\left(x_{1}, x_{2}\right)=\mathrm{E}\left\{x_{1} x_{2}\right\}
$$

Considerando o cumulante de ordem quatro, a seguinte relação pode ser estabelecida [Mendel, 1991; Picinbono, 1993]

$$
\begin{aligned}
\operatorname{cum}\left(x_{1}, x_{2}, x_{3}, x_{4}\right)=\mathrm{E} & \left\{x_{1} x_{2} x_{3} x_{4}\right\}-\mathrm{E}\left\{x_{1} x_{2}\right\} \mathrm{E}\left\{x_{3} x_{4}\right\}-\mathrm{E}\left\{x_{1} x_{3}\right\} \mathrm{E}\left\{x_{2} x_{4}\right\} \\
& -\mathrm{E}\left\{x_{1} x_{4}\right\} \mathrm{E}\left\{x_{2} x_{3}\right\}
\end{aligned}
$$

É possível observar que além do momento de ordem quatro, esse cumulante requer o conhecimento de seis valores diferentes de correlação cruzada.

Por conveniência, para variáveis aleatórias idênticas, adota-se a notação:

$$
\begin{aligned}
& \operatorname{cum}(\underbrace{x, x, x, \ldots, x}_{p \text { termos }})=\operatorname{cum}(x: p), \\
& C_{p}^{x}=\operatorname{cum}(x: p), \\
& C_{p, q}^{x}=\operatorname{cum}\left(x: p, x^{*}: q\right), \\
& C_{p, q, 1}^{x, x^{*} \mathbf{u}^{*}}=\operatorname{cum}\left(x: p, x^{*}: q, \mathbf{u}^{*}\right)
\end{aligned}
$$

e para $x$ e $\mathbf{u}$ reais, tem-se

$$
\begin{aligned}
& C_{p, q}^{x}=C_{p+q}^{x} \\
& C_{p, q, 1}^{x, x^{*} \mathbf{u}^{*}}=\boldsymbol{C}\left(x^{p+q}, \mathbf{u}\right) .
\end{aligned}
$$


Cabe observar que os cumulantes $\boldsymbol{C}_{2,1,1}^{y, y^{*} \mathbf{u}^{*}}$ e $\boldsymbol{C}\left(x^{p+q}, \mathbf{u}\right)$ são vetores coluna que possuem a mesma dimensão do vetor $\mathbf{u}$.

Os cumulantes de segunda e quarta ordens podem ser expressos como

$$
C_{1,1}^{x}=\mathrm{E}\left\{|x|^{2}\right\} \triangleq \sigma_{x}^{2}
$$

e

$$
C_{2,2}^{x}=\mathrm{E}\left\{|x|^{4}\right\}-2 \mathrm{E}\left\{|x|^{2}\right\}-\mathrm{E}\left\{x^{2}\right\} \mathrm{E}\left\{\left(x^{*}\right)^{2}\right\}
$$

sendo $\sigma_{x}^{2}$ a variância de $x$. O cumulante de ordem quatro normalizado

$$
\kappa_{x} \triangleq \frac{C_{2,2}^{x}}{\left(C_{1,1}^{x}\right)^{2}}
$$

é usualmente chamado de curtose [Picinbono, 1993]. Ele fornece uma medida de distância da "gaussianidade" de uma variável aleatória e também do achatamento de sua função densidade de probabilidade (f.d.p.). Um sinal com distribuição gaussiana é chamado de mesocúrtico e possui curtose nula. Um sinal com curtose positiva é conhecido como leptocúrtico ou super-gaussiano. Neste caso, sua f.d.p. é menos achatada que a gaussiana, apresentando um pico acentuado na média. Sinais com curtose negativa são chamados de platicúrticos ou subgaussianos e apresentam f.d.p. mais achatada que a gaussiana [Costa Neto, 2002]. A maioria dos sinais utilizados em comunicações digitais são platicúrticos, ou seja, possuem $\kappa_{x}<0$ [Shalvi \& Weinstein, 1993].

Utilizando propriedades de cumulantes, são apresentadas a seguir algumas relações para o problema da equalização. A notação é a mesma da Seção 1.3.

1. Na ausência de ruído e para canais invariantes no tempo, a matriz de autocorrelação do sinal de entrada do equalizador da Equação (1.13, página 11) pode ser escrita em função do cumulante de segunda ordem do sinal transmitido como

$$
\mathbf{R}=C_{1,1}^{a} \mathcal{H}^{H} \mathcal{H}
$$

2. O cumulante de segunda ordem do sinal de saída do equalizador pode ser expresso como

$$
C_{1,1}^{y}=\mathrm{E}\left\{|y(n)|^{2}\right\}=\mathrm{E}\left\{y^{*}(n) y(n)\right\}=\mathrm{E}\left\{\mathbf{w}^{H} \mathbf{u}^{*}(n) \mathbf{u}^{T}(n) \mathbf{w}\right\}
$$


Se o equalizador for invariante, tem-se

$$
C_{1,1}^{y}=\mathbf{w}^{H} \mathrm{E}\left\{\mathbf{u}^{*}(n) \mathbf{u}^{T}(n)\right\} \mathbf{w}=\mathbf{w}^{H} \mathbf{R} \mathbf{w} .
$$

Utilizando (2.15) e lembrando que $\mathbf{s}=\mathcal{H} \mathbf{w}$, chega-se à seguinte relação

$$
C_{1,1}^{y}=C_{1,1}^{a} \mathbf{w}^{H} \mathcal{H}^{H} \mathcal{H} \mathbf{w}=C_{1,1}^{a} \mathbf{s}^{H} \mathbf{s}
$$

3. Como conseqüência das relações anteriores, obtém-se

$$
\mathrm{E}\left\{y(n) \mathbf{u}^{*}(n)\right\}=\mathrm{E}\left\{\mathbf{u}^{*}(n) \mathbf{u}^{T}(n)\right\} \mathbf{w}=C_{1,1}^{a} \mathcal{H}^{H} \mathcal{H} \mathbf{w}=C_{1,1}^{a} \mathcal{H}^{H} \mathbf{s}
$$

4. Utilizando as propriedades de linearidade e de subconjuntos independentes (P1 e P2), o cumulante de ordem 4 do sinal de saída do equalizador pode ser escrito como [Shalvi \& Weinstein, 1994]

$$
C_{2,2}^{y}=C_{2,2}^{a} \mathbf{s}^{H} \mathbf{s}^{\odot<3>}
$$

Ele também pode ser calculado em função de momentos. Para isso, assume-se simetria circular em (2.13), obtendo-se

$$
C_{2,2}^{y}=\mathrm{E}\left\{|y(n)|^{4}\right\}-\beta \mathrm{E}\left\{|y(n)|^{2}\right\}^{2}
$$

sendo $\beta=2(=3)$ para o caso complexo (real).

5. Utilizando as relações $y(n)=\mathbf{s}^{T} \mathbf{a}(n)$ e $\mathbf{u}(n)=\mathcal{H}^{T} \mathbf{a}(n)$ e as propriedades de linearidade e de subconjuntos independentes (P1 e P2), o cumulante $\boldsymbol{C}_{2,1,1}^{y, y^{*} \mathbf{u}^{*}}$ pode ser expresso em função do cumulante de quarta ordem do sinal emitido e da resposta combinada [Shalvi \& Weinstein, 1994], ou seja,

$$
C_{2,1,1}^{y, y^{*} \mathbf{u}^{*}}=C_{2,2}^{a} \mathcal{H}^{H} \mathbf{s}^{\odot<3>}
$$

ou ainda em função de momentos

$$
C_{2,1,1}^{y, y^{*} \mathbf{u}^{*}}=\mathrm{E}\left\{|y(n)|^{2} y(n) \mathbf{u}^{*}(n)\right\}-\beta C_{1,1}^{y} \mathrm{E}\left\{y(n) \mathbf{u}^{*}(n)\right\}
$$

Na obtenção desta última relação, assume-se novamente simetria circular no caso complexo. 
6. Com o auxílio de (2.21) e (2.15), o vetor

$$
\mathcal{P}_{\mathbf{s}}{ }^{\odot<3>}=\mathcal{H}\left(\mathcal{H}^{H} \mathcal{H}\right)^{-1} \mathcal{H}^{H} \mathbf{s}^{\odot<3>}
$$

pode ser expresso em termos de cumulantes, isto é,

$$
\mathcal{P} \mathbf{s}_{n}^{\odot<3>}=\mathcal{H} C_{1,1}^{a} \mathbf{R}^{-1} \frac{C_{2,1,1}^{y, y^{*} \mathbf{u}^{*}}}{C_{2,2}^{a}}
$$

Conseqüentemente, sua norma ao quadrado pode ser calculada como

$$
\left\|\mathcal{P} \mathbf{s}_{n}^{\odot<3>}\right\|^{2}=\mathbf{s}_{n}^{\odot<3>H} \mathcal{H}\left(\mathcal{H}^{H} \mathcal{H}\right)^{-1} \mathcal{H}^{H} \mathbf{s}_{n}^{\odot<3>}=\frac{C_{1,1}^{a}}{\left(C_{2,2}^{a}\right)^{2}} \boldsymbol{C}_{2,1,1}^{y^{*}, y, \mathbf{u}^{T}} \mathbf{R}^{-1} \boldsymbol{C}_{2,1,1}^{y, y^{*} \mathbf{u}^{*}}
$$

\subsection{Funções custo associadas à equalização autodidata}

A primeira etapa do projeto de algoritmos adaptativos é a escolha de funções custo a serem minimizadas [Benveniste et al., 1987]. Dentre os critérios de otimização aplicados à equalização autodidata que são baseados em HOS, destacam-se o critério do Módulo Constante ou de Godard e o critério de Shalvi-Weinstein. O primeiro, proposto por Godard (1980), considera uma função custo de dispersão de ordem $p$ ( $p$ um inteiro positivo), definida por

$$
J_{G}=\mathrm{E}\left\{\left(|y(n)|^{p}-R_{p}^{a}\right)^{2}\right\}
$$

sendo

$$
R_{p}^{a}=\frac{\mathrm{E}\left\{|a(n)|^{2 p}\right\}}{\mathrm{E}\left\{|a(n)|^{p}\right\}}
$$

Assumindo $p=1$, chega-se à função custo proposta no trabalho pioneiro de Sato (1975). Treichler e Agee (1983) consideraram o caso $p=2$, ou seja,

$$
J_{G}=\mathrm{E}\left\{\left(|y(n)|^{2}-R_{2}^{a}\right)^{2}\right\}
$$

Shalvi e Weinstein $(1990,1993)$ propuseram um outro critério de otimização com função custo dada por

$$
J_{S W}=\frac{C_{p, q}^{y}}{\left(C_{1,1}^{y}\right)^{\frac{p+q}{2}}}
$$


sendo $C_{p, q}^{y}$ o cumulante de ordem $(p, q)$ de $y(n)$ com $p$ e $q$ inteiros positivos tal que $p+q>2$. Assumindo $p=q=2$ como em [Shalvi \& Weinstein, 1990], (2.28) se reduz a

$$
J_{S W}=\frac{C_{2,2}^{y}}{\left(C_{1,1}^{y}\right)^{2}} .
$$

A equivalência entre as funções custo de Godard e de Shalvi-Weinstein, demonstrada em [Regalia, 1999], é revisitada a seguir.

Utilizando (2.17) e (2.19), a função custo de Shalvi-Weinstein de (2.29) pode ser reescrita como

$$
J_{S W}=\frac{C_{2,2}^{a}}{\left(C_{1,1}^{a}\right)^{2}} \frac{\mathbf{s}^{H} \mathbf{s}^{\odot<3>}}{\left(\mathbf{s}^{H} \mathbf{s}\right)^{2}} .
$$

Por conveniência, escreve-se s na forma polar, ou seja, $\overline{\mathbf{s}}=\rho_{s} \mathbf{s}$, sendo $\overline{\mathbf{s}}$ o parâmetro direcional de norma unitária e $\rho_{s}=\|\mathbf{s}\|$ o parâmetro radial. Desta forma, é possível observar que $J_{S W}$ independe do parâmetro radial de $\mathbf{s}$, ou seja, $J_{S W}(\mathbf{s})=J_{S W}(\overline{\mathbf{s}})$. Em contrapartida, a função $J_{G}$ depende desse parâmetro. Assim, para se estabelecer uma relação entre $J_{G}$ e $J_{S W}$, a primeira deve ser parametrizada a fim de fazer com que as saídas dos sistemas otimizados por essas funções tenham a mesma potência [Regalia, 1999]. Para isso, o vetor de coeficientes w também deve ser escrito na forma polar, isto é,

$$
\mathbf{w}=\rho_{w} \overline{\mathbf{w}},
$$

sendo $\overline{\mathbf{w}}$ e $\rho_{w}=\|\mathbf{w}\|$ respectivamente os parâmetros direcional e radial de $\mathbf{w}$. Conseqüentemente,

$$
y(n)=\mathbf{u}^{T}(n) \mathbf{w}=\rho_{w} \mathbf{u}^{T}(n) \overline{\mathbf{w}}=\rho_{w} \bar{y}(n) .
$$

Reescrevendo (2.27) em função de $\overline{\mathbf{w}}$ e $\rho_{w}$ obtém-se

$$
J_{G}\left(\overline{\mathbf{w}}, \rho_{w}\right)=\mathrm{E}\left\{\left(\rho_{w}^{2}|\bar{y}(n)|^{2}-R_{2}^{a}\right)^{2}\right\}=\rho_{w}^{4} \mathrm{E}\left\{|\bar{y}(n)|^{4}\right\}-2 R_{2}^{a} \rho_{w}^{2} \mathrm{E}\left\{|\bar{y}(n)|^{2}\right\}+\left(R_{2}^{a}\right)^{2} .
$$

É possível observar que a função custo de Godard depende do parâmetro radial $\rho_{w}$. Derivando essa equação em relação a $\rho_{w}$ e igualando a derivada a zero, obtém-se o parâmetro radial ótimo que anula o gradiente, isto é,

$$
\rho_{\mathrm{o}}^{2}(\overline{\mathbf{w}})=\frac{R_{2}^{a} \mathrm{E}\left\{|\bar{y}(n)|^{2}\right\}}{\mathrm{E}\left\{|\bar{y}(n)|^{4}\right\}} .
$$

Cabe notar que $\rho_{\mathrm{o}}$ depende apenas do parâmetro direcional $\overline{\mathbf{w}}$. Fazendo $\rho_{w}=\rho_{\mathrm{o}}$ em (2.33), chega-se à função custo de Godard reduzida expressa por

$$
\bar{J}_{G}(\overline{\mathbf{w}})=\left(R_{2}^{a}\right)^{2}\left[1-\frac{\mathrm{E}\left\{|\bar{y}(n)|^{2}\right\}^{2}}{\mathrm{E}\left\{|\bar{y}(n)|^{4}\right\}}\right] .
$$


Essa função é uma deformação da função custo de Shalvi-Weinstein, ou seja,

$$
\bar{J}_{G}(\overline{\mathbf{w}})=\left(R_{2}^{a}\right)^{2}\left[1-\frac{1}{\beta+J_{S W}(\overline{\mathbf{w}})}\right],
$$

sendo $\beta=2(=3)$ para sinais complexos (reais).

Considerando os vetores gradientes e matrizes hessianas dessas funções custo, as seguintes relações também podem ser obtidas

$$
\nabla_{\overline{\mathbf{w}}} \bar{J}_{G}(\overline{\mathbf{w}})=\left(\frac{R_{2}^{a}}{\beta+J_{S W}(\overline{\mathbf{w}})}\right)^{2} \nabla_{\overline{\mathbf{w}}} J_{S W}(\overline{\mathbf{w}})
$$

e

$$
\mathbf{F}_{G}(\overline{\mathbf{w}})=\left(R_{2}^{a}\right)^{2}\left[\frac{\mathbf{F}_{S W}(\overline{\mathbf{w}})}{\left(\beta+J_{S W}(\overline{\mathbf{w}})\right)^{2}}-2 \frac{\nabla_{\overline{\mathbf{w}}} J_{S W}^{*}(\overline{\mathbf{w}}) \nabla_{\overline{\mathbf{w}}} J_{S W}^{H}(\overline{\mathbf{w}})}{\left(\beta+J_{S W}(\overline{\mathbf{w}})\right)^{3}}\right] .
$$

A partir de (2.37) pode-se verificar que a deformação que leva o critério de Godard reduzido ao critério de Shalvi-Weinstein é monotônica, o que garante que uma modificação no parâmetro direcional $\overline{\mathbf{w}}$ provoque o mesmo efeito nos dois critérios e conseqüentemente seus pontos estacionários coincidem. Além disso, a classificação de cada ponto estacionário como mínimo local, mínimo global, máximo local ou ponto de sela é igualmente conservada [Regalia, 1999]. Desta forma, pode-se dizer que os critérios de Godard e de Shalvi-Weinstein são teoricamente equivalentes. As demonstrações de (2.36) e (2.37) são feitas em [Regalia, 1999]. A demonstração de (2.38) é apresentada no Apêndice B.

Para exemplificar a equivalência dos critérios, considera-se um canal IIR (Infinite Impulse Response) com função de transferência [Ding \& Li, 2001]

$$
\mathrm{H}_{1}(z)=\frac{1}{1+0,6 z^{-1}}
$$

Para $\mathrm{H}_{1}$ e 2-PAM, as funções custo de Godard, Shalvi-Weinstein e Godard reduzida são mostradas na Figura 2.1. Neste caso, a função custo de Godard possui 2 mínimos globais, 2 mínimos locais e 1 máximo local indicados na figura. É possível observar que a função de Godard reduzida é uma deformação da função de Shalvi-Weinstein e que seus pontos estacionários coincidem. 
a)

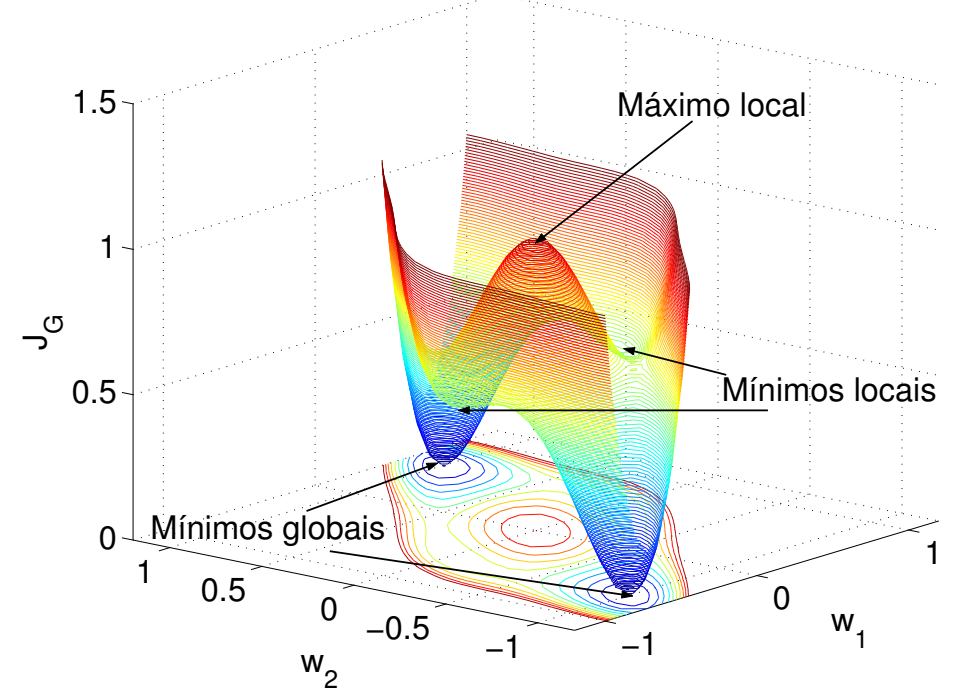

b)

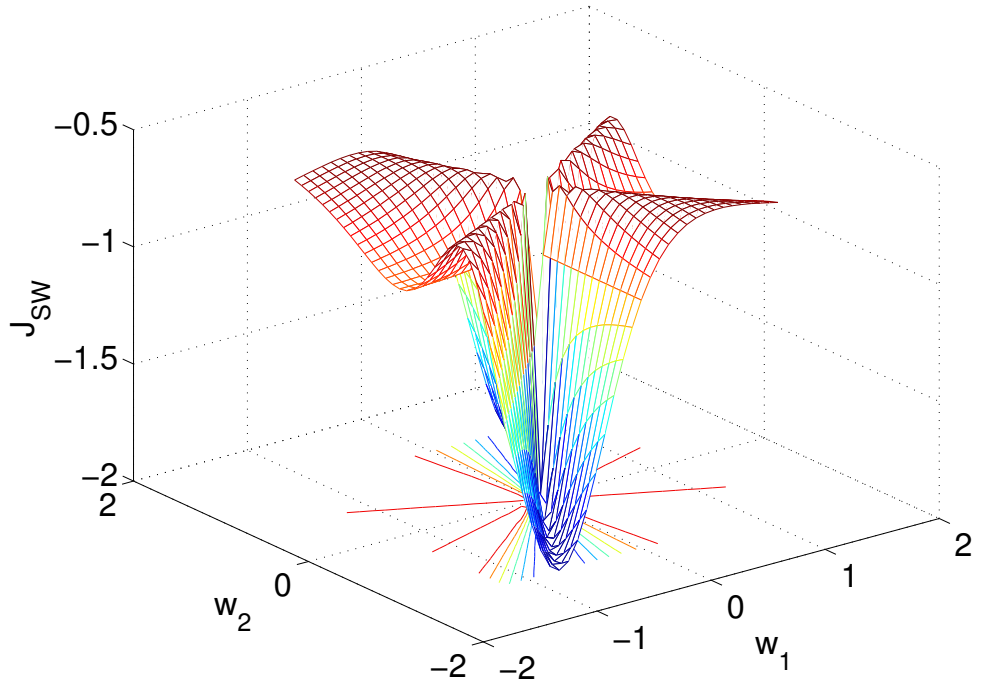

c)

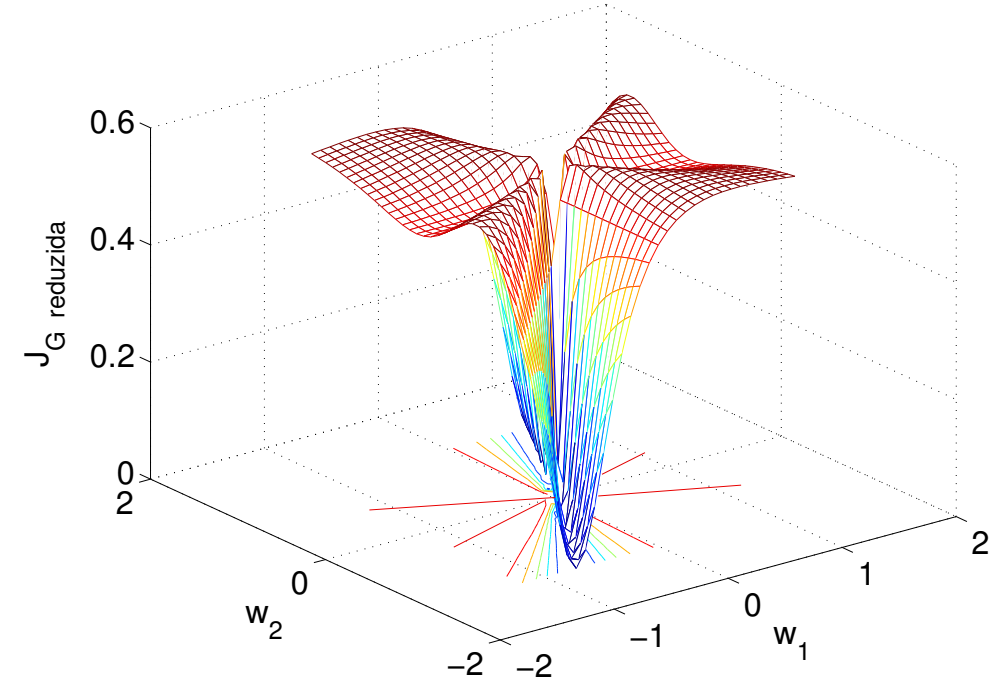

Figura 2.1: Funções custo a) Godard, b) Shalvi-Weinstein e c) Godard reduzida; 2-PAM, canal H. $_{1}$. 


\subsection{Gradientes e pontos estacionários}

As funções custo (2.27) e (2.29) e seus respectivos gradientes são apresentados na Tabela 2.1. Os cálculos efetuados na obtenção dos gradientes estão no Apêndice B. Para compactar a notação, os índices de tempo foram suprimidos. Cabe observar que o gradiente de $J_{S W}$ depende do escalar $R_{2}^{y}$ definido como

$$
R_{2}^{y} \triangleq \frac{\mathrm{E}\left\{|y(n)|^{4}\right\}}{\mathrm{E}\left\{|y(n)|^{2}\right\}}
$$

\begin{tabular}{|c|c|c|}
\hline Função & Godard & Shalvi-Weinstein \\
Custo & $J_{G}=\mathrm{E}\left\{\left(|y|^{2}-R_{2}^{a}\right)^{2}\right\}$ & $J_{S W}=\frac{C_{2,2}^{y}}{\left(C_{1,1}^{y}\right)^{2}}$ \\
\hline Gradiente & $\nabla_{\mathbf{w}} J_{G}=2 \bar{\beta} \mathrm{E}\left\{\left(|y|^{2}-R_{2}^{a}\right) y \mathbf{u}^{*}\right\}$ & $\nabla_{\mathbf{w}} J_{S W}=\frac{2 \bar{\beta}}{\mathrm{E}\left\{|y|^{2}\right\}^{2}} \mathrm{E}\left\{\left(|y|^{2}-R_{2}^{y}\right) y \mathbf{u}^{*}\right\}$ \\
\hline
\end{tabular}

Tabela 2.1: Gradientes das funções custo consideradas; $\bar{\beta}=\beta-1$.

Utilizando as relações $(2.20),(2.19)$ e $(2.17)$, os escalares $R_{2}^{a}$ e $R_{2}^{y}$ podem ser reescritos como

$$
R_{2}^{a}=\frac{C_{2,2}^{a}}{C_{1,1}^{a}}+\beta C_{1,1}^{a}
$$

e

$$
R_{2}^{y}=\frac{C_{2,2}^{y}}{C_{1,1}^{y}}+\beta C_{1,1}^{y}=\frac{C_{2,2}^{a} \mathbf{s}^{H} \mathbf{s}^{\odot<3>}}{C_{1,1}^{a} \mathbf{s}^{H} \mathbf{s}}+\beta C_{1,1}^{a} \mathbf{s}^{H} \mathbf{s} .
$$

Comparando as equações (2.40) e (2.41), é possível observar que $R_{2}^{y}=R_{2}^{a}$ somente quando $\mathbf{s}^{H} \mathbf{S}^{\odot<3>}\left(\mathbf{s}^{H} \mathbf{s}\right)^{-1}=\mathbf{s}^{H} \mathbf{S}=1$, o que só é possível no caso de equalização perfeita, ou seja, quando $\mathbf{s}=\left[\begin{array}{lllllll}0 & \cdots & 0 & \mathrm{e}^{j \theta} & 0 & \cdots & 0\end{array}\right]^{T}$. Embora a igualdade entre esses escalares só seja obtida nessa situação, é comum encontrar implicitamente na literatura aproximações do tipo $R_{2}^{y} \approx R_{2}^{a}$ e $C_{2}^{y} \approx C_{2}^{a}$ [Godard, 1980; Shalvi \& Weinstein, 1990]. Isso é feito para evitar a necessidade de se estimar $R_{2}^{y}$ ou $C_{2}^{y}$, obtendo-se assim algoritmos mais simples. Desta forma, ao se considerar $R_{2}^{y}=R_{2}^{a}$ e $C_{2}^{y}=C_{2}^{a}$ no gradiente de Shalvi-Weinstein da Tabela 2.1, obtém-se a seguinte relação

$$
\nabla_{\mathbf{w}} J_{G}(n)=\left.\mathrm{E}\left\{|a(n)|^{2}\right\}^{2} \nabla_{\mathbf{w}} J_{S W}(n)\right|_{R_{2}^{y}=R_{2}^{a}} .
$$


Os pontos estacionários das funções custo de Godard e de Shalvi-Weinstein satisfazem a Proposição 1 mostrada a seguir. Eles são identificados pelo índice $e$.

Proposição 1 Sejam as funções custo de Godard e de Shalvi-Weinstein definidas respectivamente em (2.27) e (2.29). Assumindo-se que $\mathcal{P} \mathbf{s}_{e}=\mathbf{s}_{e}$, seus pontos estacionários satisfazem uma equação do tipo super-exponencial dada por

$$
\mathbf{s}_{e}=\alpha \mathcal{P} \mathbf{s}_{e}^{\odot<3>}
$$

sendo $\mathcal{P}$ o operador de projeção definido em (1.14) e a um escalar que depende da função custo considerada. Para a função custo de Shalvi-Weinstein tem-se

$$
\alpha=\alpha_{S W}=\frac{\gamma^{a}}{\gamma^{y}}=\frac{C_{2,2}^{a}}{C_{1,1}^{a}} \frac{C_{1,1}^{y}}{C_{2,2}^{y}}
$$

e para a função custo de Godard

$$
\alpha=\alpha_{G}=\frac{\gamma^{a}}{\beta C_{1,1}^{y}-R_{2}^{a}}=-\frac{C_{2,2}^{a}}{C_{1,1}^{a}\left(\beta C_{1,1}^{y}-R_{2}^{a}\right)} .
$$

No dominio dos coeficientes do equalizador, (2.43) se reduz a

$$
\mathbf{w}_{e}=-\frac{\alpha}{\gamma^{a}} \mathbf{R}^{-1} \boldsymbol{C}_{2,1,1}^{y, y^{*} \mathbf{u}^{*}}
$$

Demonstração: Utilizando as relações (2.22), (2.21) e (2.18), o vetor gradiente da função custo de Shalvi-Weinstein da Tabela 2.1 pode ser reescrito como

$$
\begin{aligned}
\nabla_{\mathbf{w}} J_{S W} & =\frac{2 \bar{\beta}}{\left(C_{1,1}^{y}\right)^{2}}\left[C_{2,1,1}^{y, y^{*} \mathbf{u}^{*}}+\left(\beta C_{1,1}^{y}-R_{2}^{y}\right) \mathrm{E}\left\{y(n) \mathbf{u}^{*}(n)\right\}\right] \\
& =\frac{2 \bar{\beta}}{\left(C_{1,1}^{y}\right)^{2}}\left[C_{2,2}^{a} \boldsymbol{H}^{H} \mathbf{s}^{\odot<3>}+\left(\beta C_{1,1}^{y}-R_{2}^{y}\right) C_{1,1}^{a} \boldsymbol{H}^{H} \mathbf{s}\right]
\end{aligned}
$$

Definindo

$$
\gamma^{a} \triangleq-\frac{C_{2,2}^{a}}{C_{1,1}^{a}}=\beta C_{1,1}^{a}-R_{2}^{a}
$$

$e$

$$
\gamma^{y} \triangleq-\frac{C_{2,2}^{y}}{C_{1,1}^{y}}=\beta C_{1,1}^{y}-R_{2}^{y}
$$


e utilizando essas definições em (2.47), obtém-se

$$
\nabla_{\mathbf{w}} J_{S W}=\frac{2 \bar{\beta} C_{1,1}^{a}}{\left(C_{1,1}^{y}\right)^{2}}\left[-\gamma^{a} \mathcal{H}^{H} \mathbf{s}^{\odot<3>}+\gamma^{y} \mathcal{H}^{H} \mathbf{s}\right] .
$$

Igualando esse gradiente a $\mathbf{0}$, chega-se a

$$
\gamma^{y} \mathcal{H}^{H} \mathbf{s}_{e}=\gamma^{a} \mathcal{H}^{H} \mathbf{s}_{e}^{\odot<3>}
$$

Multiplicando ambos os lados de (2.51) por $\mathcal{H}\left(\mathcal{H}^{H} \mathcal{H}\right)^{-1}$, obtém-se a relação

$$
\mathbf{s}_{e}=\underbrace{\frac{\gamma^{a}}{\gamma^{y}}}_{\alpha_{S W}} \mathcal{P} \mathbf{s}_{e}^{\odot<3>}
$$

que é satisfeita pelos pontos estacionários da função custo de Shalvi-Weinstein. Esse resultado foi obtido originalmente em [Regalia \& Mboup, 1999, Th.6]. No caso do gradiente da função custo de Godard, fazendo $R_{2}^{y}=R_{2}^{a}$ e $C_{2}^{y}=C_{2}^{a}$ e aplicando (2.42) em (2.47), chega-se a

$$
\begin{aligned}
\nabla_{\mathbf{w}} J_{G} & =2 \bar{\beta}\left[C_{2,2}^{a} \mathcal{H}^{H} \mathbf{s}^{\odot<3>}+\left(\beta C_{1,1}^{y}-R_{2}^{a}\right) C_{1,1}^{a} \mathcal{H}^{H} \mathbf{s}\right] \\
& =2 \bar{\beta} C_{1,1}^{a}\left[-\gamma^{a} \mathcal{H}^{H} \mathbf{s}^{\odot<3>}+\left(\beta C_{1,1}^{y}-R_{2}^{a}\right) \mathcal{H}^{H} \mathbf{s}\right]
\end{aligned}
$$

Igualando esse gradiente a $\mathbf{0}$ e multiplicando ambos os lados da equação resultante por $\mathcal{H}\left(\mathcal{H}^{H} \mathcal{H}\right)^{-1}$, obtém-se a seguinte relação para os pontos estacionários da função custo de Godard

$$
\mathbf{s}_{e}=\underbrace{\frac{\gamma^{a}}{\beta C_{1,1}^{y}-R_{2}^{a}}}_{\alpha_{G}} \mathcal{P} \mathbf{s}_{e}^{\odot<3>},
$$

o que completa a demonstração no domínio da resposta combinada. Para demonstrar (2.46), utiliza-se a definição do operador de projeção, lembrando que $\mathbf{s}_{e}=\mathcal{H}_{\mathbf{w}_{e}}$. Desta forma, (2.43) pode ser reescrita como

$$
\mathcal{H} \mathbf{w}_{e}=\alpha \mathcal{H}\left(\mathcal{H}^{H} \mathcal{H}\right)^{-1} \mathcal{H}^{H} \mathbf{s}^{\odot<3>}
$$

Utilizando (2.19) e (2.15) em (2.55), chega-se a

$$
\mathcal{H} \mathbf{w}_{e}=\alpha \frac{C_{1,1}^{a}}{C_{2,2}^{a}} \mathcal{H} \mathbf{R}^{-1} C_{2,1,1}^{y, y^{*} \mathbf{u}^{*}}
$$

Multiplicando ambos os lados dessa equação por $\left(\mathcal{H}^{H} \mathcal{H}\right)^{-1} \mathcal{H}^{H}$ obtém-se $(2.46)$.

Cabe observar que fazendo $R_{2}^{y}=R_{2}^{a}$ e $C_{1,1}^{y}=C_{1,1}^{a}$, obtém-se a igualdade $\alpha_{G}=\alpha_{S W}$ e os pontos estacionários das funções custo de Godard e de Shalvi-Weinstein coincidem. 


\subsection{Algoritmos do gradiente determinístico}

Após a definição das funções custo, deve-se escolher um método de minimização [Benveniste et al., 1987]. Desta forma, são obtidos a seguir, algoritmos autodidatas do gradiente determinístico a partir das funções custo de Godard e de Shalvi-Weinstein.

O método do gradiente determinístico, também conhecido na literatura como Steepest Descent [Sayed, 2003], minimiza uma função custo $J(\mathbf{w})$ através do algoritmo iterativo

$$
\mathbf{w}(n)=\mathbf{w}(n-1)-\alpha(n) \nabla_{\mathbf{w}} J(n),
$$

sendo $\alpha(n)$ um passo de adaptação positivo. Assim, o vetor de coeficientes $\mathbf{w}(n)$ é atualizado a partir de uma correção do vetor de coeficientes do instante anterior $\mathbf{w}(n-1)$ na direção negativa do gradiente $\nabla_{\mathbf{w}} J(n)$.

Considerando o vetor gradiente da função custo de Godard (Tabela 2.1) e um escalar como passo de adaptação, ou seja, fazendo $\alpha(n)=\mu(n)$, obtém-se o algoritmo do Módulo Constante baseado no gradiente determinístico, denotado por SD-CMA (Steepest-Descent Constant Modulus Algorithm) [Godard, 1980; Treichler \& Agee, 1983]. Ele pode ser descrito pela seguinte equação

$$
\mathbf{w}(n)=\mathbf{w}(n-1)-\mu(n) \mathrm{E}\left\{e(n) \mathbf{u}^{*}(n)\right\}
$$

sendo

$$
e(n) \triangleq\left(|y(n)|^{2}-R_{2}^{a}\right) y(n)
$$

e

$$
y(n)=\mathbf{u}^{T}(n) \mathbf{w}(n-1) .
$$

A constante $2 \bar{\beta}$ que aparece em $\nabla_{\mathbf{w}} J_{G}$ foi incorporada ao passo de adaptação $\mu(n)$. Além disso, é comum considerar um passo de adaptação constante $\mu$.

Por sua vez, o algoritmo de Shalvi-Weinstein baseado no gradiente determinístico, denotado SD-SWA (Steepest-Descent Shalvi-Weinstein Algorithm), pode ser descrito por

$$
\mathbf{w}(n)=\mathbf{w}(n-1)-\frac{\mathbf{R}^{-1}}{\gamma^{a}} \mathrm{E}\left\{e(n) \mathbf{u}^{*}(n)\right\} .
$$

Esse algoritmo é obtido na Proposição 2, mostrada a seguir. 
Proposição 2 Assumindo-se que $C_{1,1}^{y}=C_{1,1}^{a}$, o algoritmo de Shalvi-Weinstein baseado no gradiente determinístico (SD-SWA) da Equação (2.61) pode ser obtido pelo método do gradiente, utilizando $\nabla_{\mathbf{w}} J_{G}$ e um passo de adaptação ótimo para velocidade de convergência.

Demonstração: Utilizando as relações (2.22) e (2.18), o gradiente da função custo de Godard (Tabela 2.1) pode ser reescrito como

$$
\boldsymbol{\nabla}_{\mathbf{w}} J_{G}(n)=2(\beta-1)\left[\boldsymbol{C}_{2,1,1}^{y, y^{*} \mathbf{u}^{*}}+\left(\beta C_{1,1}^{y}-R_{2}^{a}\right) \mathbf{R w}(n-1)\right] .
$$

Considerando $C_{1,1}^{y}=C_{1,1}^{a}$ e a definição de $\gamma^{a}$ dada em (2.48), chega-se a

$$
\nabla_{\mathbf{w}} J_{G}(n)=2(\beta-1)\left[\boldsymbol{C}_{2,1,1}^{y, y^{*} \mathbf{u}^{*}}+\gamma^{a} \mathbf{R} \mathbf{w}(n-1)\right]
$$

Substituindo esse gradiente em (2.57) e incorporando a constante $2(\beta-1)$ no passo de adaptação, obtém-se

$$
\mathbf{w}(n)=\left(\mathbf{I}-\alpha(n) \gamma^{a} \mathbf{R}\right) \mathbf{w}(n-1)-\alpha(n) \boldsymbol{C}_{2,1,1}^{y, y^{*} \mathbf{u}^{*}}
$$

Escolhendo o passo de adaptação

$$
\alpha=\frac{\mathbf{R}^{-1}}{\gamma^{a}}
$$

o algoritmo (2.64) se reduz a

$$
\mathbf{w}(n)=-\frac{\mathbf{R}^{-1}}{\gamma^{a}} C_{2,1,1}^{y, y^{*} \mathbf{u}^{*}}
$$

A dependência do tempo foi suprimida em $\alpha$, dado que para sinais estacionários $\mathbf{R}(n)=\mathbf{R}$. Cabe notar que o cumulante $\boldsymbol{C}_{2,1,1}^{y, y^{*} \mathbf{u}^{*}}$ depende de $\mathbf{w}(n-1)$ e que (2.66) atinge um ponto estacionário da função custo de Godard após algumas iterações. Desta forma, (2.65) é o passo ótimo para velocidade de convergência. Substituindo (2.65) e $\nabla_{\mathbf{w}} J_{G}$ da Tabela 2.1 em (2.57), chega-se a (2.61), o que completa a demonstração.

O passo ótimo para velocidade de convergência foi obtido em [Mboup \& Regalia, 2000], sendo um escalar no domínio da resposta combinada. No domínio dos coeficientes do equalizador, ele se torna uma matriz e é dado por (2.65). Comparando as equações (2.61) e (2.58), conclui-se que o SD-SWA difere do SD-CMA apenas pelo passo de adaptação. O algoritmo de Shalvi-Weinstein é igual ao algoritmo do Módulo Constante com passo ótimo para velocidade de convergência dado por (2.65) e ambos podem ser obtidos a partir da minimização 
da função custo de Godard como é estabelecido na Proposição 2. Além disso, fazendo-se a seguinte aproximação para a hessiana da função custo de Godard

$$
\mathrm{E}\left\{\left(\beta|y(n)|^{2}-R_{2}^{a}\right) \mathbf{u}^{*}(n) \mathbf{u}^{T}(n)\right\} \approx \gamma^{a} \mathrm{E}\left\{\mathbf{u}^{*}(n) \mathbf{u}^{T}(n)\right\}=\gamma^{a} \mathbf{R},
$$

o SD-SWA também pode ser interpretado como um algoritmo do tipo Newton. Como os métodos para garantir a positividade da matriz de autocorrelação são bem conhecidos na literatura [Haykin, 1996], pode ser vantajoso considerar essa aproximação [Silva et al., 2004e].

A matriz $\mathbf{R}^{-1}$ efetua um pré-branqueamento do sinal $u(n)$. Desta forma, espera-se que como no caso supervisionado, essa matriz vezes o vetor gradiente torne a convergência do SD-SWA atrativa próximo a um ponto de mínimo da função custo. No entanto, para que essa característica seja válida em toda a superfície de desempenho, deve-se introduzir um passo de adaptação $0<\beta_{S W}(n)<1$ [Luenberger, 1984, p. 226]. Considerando $\beta_{S W}$ constante e interpretando o SD-SWA como um algoritmo do gradiente, o passo ótimo para velocidade de convergência passa a ser

$$
\alpha=\beta_{S W} \mathbf{R}^{-1} / \gamma^{a} .
$$

Cabe observar ainda que é possível obter o SD-SWA através de algoritmos do tipo superexponencial no domínio dos coeficientes do equalizador. Isso será mostrado na Proposição 5 da Seção 2.6.

\subsection{O algoritmo Super-Exponencial no domínio da res- posta combinada}

O algoritmo Super-Exponencial no domínio da resposta combinada é revisitado. Em seguida, é feita uma extensão dos resultados da análise de convergência do algoritmo do Gradiente Projetado de [Mboup \& Regalia, 2000].

Se o único componente dominante de $\mathbf{s}_{n}$ está na posição $\tau_{d}$, então a resposta combinada ideal pode ser aproximada pela $m$-ésima potência de Hadamard $\left(\mathbf{s}_{n} / s_{n, \tau_{d}}\right)^{\odot m}$ quando $m \longrightarrow$ $\infty$ [Mboup \& Regalia, 2000]. Como exemplo, considera-se o algoritmo iterativo

$$
\begin{aligned}
\widetilde{\mathbf{s}} & =\mathbf{s}_{n-1}^{\odot 3} \\
\mathbf{s}_{n} & =\frac{\widetilde{\mathbf{s}}}{\|\widetilde{\mathbf{s}}\|},
\end{aligned}
$$


cujo comportamento é ilustrado na Figura 2.2. Partindo do vetor $\mathbf{s}_{0}$ com termo dominante na posição 3 e ISI = 2,4673 dB (Figura 2.2-a), após duas iterações obtém-se o resultado da Figura 2.2-c. Continuando com mais duas iterações, chega-se à resposta combinada ideal $\mathbf{s}_{5}=\left[\begin{array}{llllllllll}0 & 0 & 1 & 0 & 0 & 0 & 0 & 0 & 0 & 0\end{array}\right]^{T}$ e a ISI residual se anula (ISI $\left.=-\infty \mathrm{dB}\right)$. Esse algoritmo sempre converge para a resposta combinada ideal, desde que o vetor de inicialização tenha um único componente dominante. Uma vez atingida a resposta ideal, a posição do elemento não-nulo depende da condição inicial (máximo do módulo da resposta combinada no instante inicial). Na prática, como a resposta combinada depende do canal, o procedimento iterativo da Figura 2.2 só tem interesse acadêmico [Regalia \& Mboup, 1999].

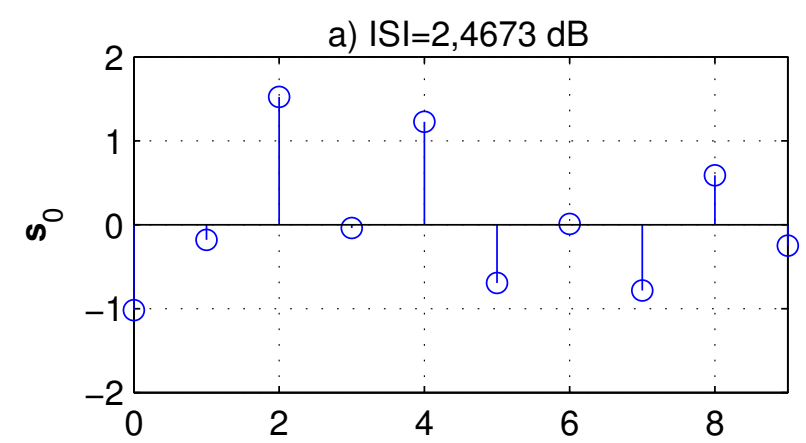

b) $|S|=-4,0091 \mathrm{~dB}$

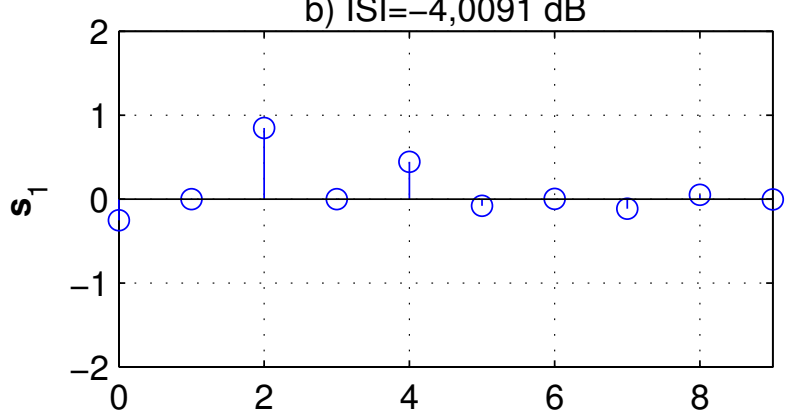

c) $|S|=-16,6144 \mathrm{~dB}$

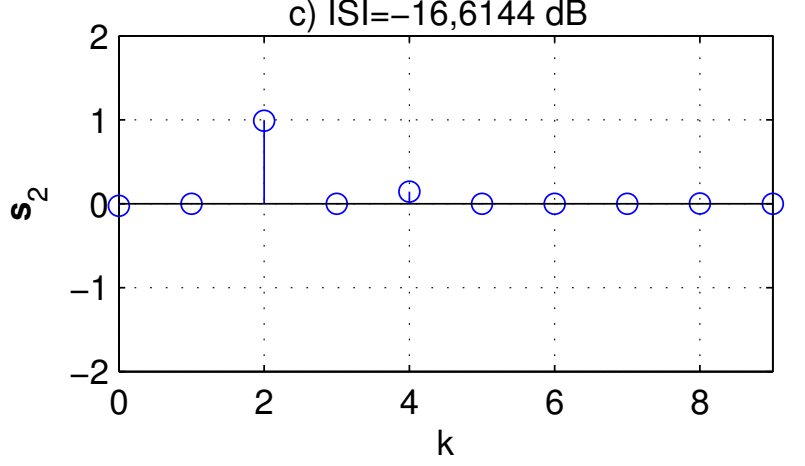

Figura 2.2: Comportamento do algoritmo no domínio da resposta combinada ao longo do tempo:

a) inicialização, b) primeira iteração, c) segunda iteração. 
Da Equação (1.9, página 10), verifica-se que o vetor de resposta combinada se restringe ao espaço $\mathcal{S}$ formado pelas colunas da matriz de convolução do canal. Conseqüentemente, o algoritmo (2.67) deve ser modificado considerando o operador de projeção ortogonal sobre $\mathcal{S}$, definido na Equação (1.14, página 11). Essa observação leva ao algoritmo Super-Exponencial proposto por Shalvi e Weinstein (1990) e descrito pelas seguintes equações

$$
\begin{aligned}
\widetilde{\mathbf{s}} & =\mathcal{P} \mathbf{s}_{n-1}^{\odot q} \\
\mathbf{s}_{n} & =\frac{\widetilde{\mathbf{s}}}{\|\widetilde{\mathbf{s}}\|},
\end{aligned}
$$

sendo $q$ um número ímpar, $q=2 p-1 \operatorname{com} p>1$. O algoritmo (2.68) pode ser obtido a partir da maximização da função custo [Regalia \& Mboup, 1999]

$$
f\left(\mathbf{s}_{n-1}\right)=\frac{\mathbf{s}_{n-1}^{T} \mathbf{s}_{n-1}^{\odot q}}{\left(\mathbf{s}_{n-1}^{T} \mathbf{s}_{n-1}\right)^{\frac{q+1}{2}}} .
$$

Essa função pertence à família de funções objetivo de Donoho e satisfaz as seguintes propriedades

$$
f\left(\alpha \mathbf{s}_{n}\right)=f\left(\mathbf{s}_{n}\right)
$$

para qualquer $\alpha \neq 0$ e

$$
0<f\left(\mathbf{s}_{n}\right) \leq 1
$$

para todo $\mathbf{s}_{n} \in \ell_{2}$ [Donoho, 1981; Mboup \& Regalia, 2000].

Assumindo $q=3$ em (2.69), a função custo de Shalvi-Weinstein de (2.30) pode ser reescrita como

$$
J_{S W}\left(\mathbf{s}_{n-1}\right)=\frac{C_{2,2}^{a}}{\left(C_{1,1}^{a}\right)^{2}} f\left(\mathbf{s}_{n-1}\right) .
$$

Se $C_{2,2}^{a}<0$, condição válida para a maioria dos sinais utilizados em comunicações digitais, o sinal de $J_{S W}$ é contrário ao de $f\left(\mathbf{s}_{n-1}\right)$, e a partir de $(2.71)$ e $(2.72)$ pode-se escrever

$$
\frac{C_{2,2}^{a}}{\left(C_{1,1}^{a}\right)^{2}} \leq J_{S W}\left(\mathbf{s}_{n}\right)<0 .
$$

Essa relação pode ser observada no exemplo da Figura 2.1-b, página 43.

Combinando as equações (2.68a) e (2.68b) e generalizando para sinais complexos, chegase a

$$
\mathbf{s}_{n}=\frac{\mathcal{P} \mathbf{s}_{n-1}^{\odot<2 p-1>}}{\left\|\mathcal{P} \mathbf{s}_{n-1}^{\odot<2 p-1>}\right\|}
$$


Na ausência de ruído, o algoritmo Super-Exponencial sempre converge para a situação de equalização perfeita (condição de zero-forcing), desde que $\mathcal{P}=\mathbf{I}$, o que representa o caso da ordem suficiente. Quanto maior o valor de $p$, menor será o número de iterações necessárias para o algoritmo convergir [Regalia \& Mboup, 1999].

Assumindo caso real e $p=2$, obtém-se

$$
\mathbf{s}_{n}=\frac{\mathcal{P} \mathbf{s}_{n-1}^{\odot 3}}{\left\|\mathcal{P} \mathbf{s}_{n-1}^{\odot 3}\right\|}
$$

Neste caso, a função custo é dada por

$$
f\left(\mathbf{s}_{n-1}\right)=\frac{\mathbf{s}_{n-1}^{T} \mathbf{s}_{n-1}^{\odot 3}}{\left(\mathbf{s}_{n-1}^{T} \mathbf{s}_{n-1}\right)^{2}} .
$$

A partir de (2.75), pode-se obter uma relação para os pontos estacionários do algoritmo Super-Exponencial. Comparando com as relações obtidas na Proposição 1 para as funções custo de Godard e de Shalvi-Weinstein, conclui-se que há uma equivalência entre elas. Devese notar que os pontos estacionários diferem apenas por um fator de escala.

A seguir, revisita-se a interpretação do algoritmo Super-Exponencial como um algoritmo do Gradiente Projetado [Mboup \& Regalia, 2000].

\section{O algoritmo do Gradiente Projetado (GP)}

Considera-se sem perda de generalidade o algoritmo da Equação (2.75). A extensão para o caso complexo e $p>2$ é direta. Seja $\mathcal{S}$ um subespaço de vetores de $\mathbb{R}^{M+N-1}$ e $\mathcal{P}$ o operador de projeção ortogonal de $\mathbb{R}^{M+N-1}$ em $\mathcal{S}$, definido em (1.14, página 11). Supondo que $f_{n-1}$ é diferenciável em $\mathbf{s}_{n-1}$ e utilizando as definições de gradiente projetado [Guidorizzi, 1986], o algoritmo (2.75) pode ser interpretado como um algoritmo do gradiente através das seguintes equações

$$
\begin{aligned}
\mathbf{v} & =\mathbf{s}_{n-1}+\mu_{n} \mathcal{P} \nabla f_{n-1}, \\
\mathbf{s}_{n} & =\frac{\mathbf{v}}{\|\mathbf{v}\|},
\end{aligned}
$$

sendo

$$
\mathcal{P} \nabla f_{n-1}=\frac{1}{\left(\mathbf{s}_{n-1}^{T} \mathbf{s}_{n-1}\right)^{2}} \mathcal{P}\left[\mathbf{s}_{n-1}^{\odot 3}-\left(f_{n-1} \mathbf{s}_{n-1}^{T} \mathbf{s}_{n-1}\right) \mathbf{s}_{n-1}\right]
$$


e $\mu_{n}$ um passo de adaptação variável. Esse algoritmo é idêntico ao (2.75) quando

$$
\mu_{n}=\frac{\mathbf{s}_{n-1}^{T} \mathbf{s}_{n-1}}{f_{n-1}} .
$$

Evidentemente, para que isso seja verdade, deve-se considerar a Equação (2.77b), o que sempre garante $\left\|\mathbf{s}_{n}\right\|=1$. Além disso, o algoritmo (2.77) deve ser inicializado com um vetor de resposta combinada de norma unitária $\left(\left\|s_{0}\right\|=1\right)$ pertencente ao espaço $\mathcal{S}$ [Mboup \& Regalia, 2000]. Cabe observar ainda que $\mu_{n}$ de (2.79) é o passo ótimo para velocidade de convergência desse algoritmo. Utilizando esse passo, as relações

$$
0<f_{n-1}<\left\|\mathcal{P} \mathbf{s}_{n-1}^{\odot 3}\right\|<f_{n}<\left\|\mathcal{P}_{n}^{\odot 3}\right\| \leq 1
$$

e

$$
f_{n}-f_{n-1}>4\left(\left\|\mathcal{P} \mathbf{s}_{n-1}^{\odot 3}\right\|-f_{n-1}\right)
$$

são válidas, exceto num ponto estacionário, pois, neste caso, ocorrem as igualdades $f_{n-1}=$ $\left\|\mathcal{P} \mathbf{s}_{n-1}^{\odot 3}\right\|=f_{n}=\left\|\mathcal{P} \mathbf{s}_{n}^{\odot 3}\right\|$. A convergência do algoritmo é uma conseqüência direta da seqüência $\left\{f_{n}\right\}_{n \geq 0}$ que aumenta estritamente e é limitada. Além disso, a partir de (2.80) é possível observar que as seqüências $\left\{f_{n}\right\}_{n \geq 0}$ e $\left\{\left\|\mathcal{P}_{\mathbf{s}_{n}}^{\odot 3}\right\|\right\}_{n \geq 0}$ convergem com a mesma taxa para o mesmo limite [Mboup \& Regalia, 2000].

\section{O algoritmo do Gradiente Projetado não-normalizado}

Inspirado-se no algoritmo (2.77), é possível reescrever (2.75) de forma recorrente inserindo a normalização no termo de atualização, ou seja,

$$
\mathbf{s}_{n}=\mathbf{s}_{n-1}+\rho_{n}^{-1} \mathcal{P}\left(\mathbf{s}_{n-1}^{\odot 3}-\rho_{n} \mathbf{s}_{n-1}\right)
$$

com $\rho_{n}=\left\|\mathcal{P}_{\mathbf{s}_{n-1}}{ }^{\odot 3}\right\|$. Observando essa equação, pode-se considerar de forma mais geral um algoritmo não-normalizado descrito por

$$
\mathbf{s}_{n}=\mathbf{s}_{n-1}+\mu_{n} \mathcal{P}\left(\mathbf{s}_{n-1}^{\odot 3}-\alpha_{n} \mathbf{s}_{n-1}\right)
$$

sendo $\alpha_{n}$ um escalar ajustável e $\mu_{n}$ o passo de adaptação. A idéia é obter um algoritmo mais geral que o (2.77). Neste caso, $\alpha_{n}$ tem um papel fundamental, podendo assumir valores diferentes, conforme o algoritmo considerado. Por exemplo, partindo de (2.83) é possível chegar à (2.77), assumindo

$$
\alpha_{n}=f_{n-1}
$$


e

$$
\left\|\mathbf{s}_{n}\right\|=1
$$

Para assegurar a norma unitária, deve-se utilizar a Equação (2.77b). Na Proposição 5 da Seção 2.6, será dado um outro exemplo, em que se obtém o algoritmo SD-SWA fazendo $\alpha_{n}=1$ e algumas considerações adicionais no algoritmo não-normalizado no domínio dos coeficientes do equalizador.

\section{O intervalo do passo de adaptação do algoritmo do GP não-normalizado}

Inspirando-se em [Mboup \& Regalia, 2000], uma análise de convergência do algoritmo (2.83) é apresentada no Teorema 1, mostrado a seguir.

Teorema 1 Considerando o operador de projeção definido na Equação (1.14, página 11) e $\mathcal{P} \mathbf{s}=\mathbf{s}$, o algoritmo do Gradiente Projetado não-normalizado da Equação (2.83) terá convergência assegurada se para cada iteração n, o passo de adaptação $\mu_{n}$ estiver no intervalo

$$
0<\mu_{n}<\frac{2 f_{n-1}}{2 \alpha_{n} f_{n-1}-\frac{\left\|\mathcal{P}_{n-1}^{\odot 3}\right\|^{2}}{\left(\mathbf{s}_{n-1}^{T} \mathbf{s}_{n-1}\right)^{2}}}=\frac{2 \mathbf{s}_{n-1}^{T} \mathbf{s}_{n-1}^{\odot 3}}{2 \alpha_{n} \mathbf{s}_{n-1}^{T} \mathbf{s}_{n-1}^{\odot 3}-\mathbf{s}_{n-1}^{\odot 3 T} \mathcal{P} \mathbf{s}_{n-1}^{\odot 3}} .
$$

Demonstração: Reescrevendo $f_{n-1}$ em função do vetor de norma unitária $\overline{\mathbf{s}}=\mathbf{s} /\|\mathbf{s}\|$, obtém-se

$$
f_{n-1}=\frac{\mathbf{s}_{n-1}^{T} \mathbf{s}_{n-1}^{\odot 3}}{\left(\mathbf{s}_{n-1}^{T} \mathbf{s}_{n-1}\right)^{2}}=\overline{\mathbf{s}}_{n-1}^{T} \overline{\mathbf{s}}_{n-1}^{\odot 3} .
$$

Uma condição para que $f_{n-1}$ seja convexa é [Luenberger, 1984, p. 178]

$$
f_{n}-f_{n-1} \geqslant\left[\overline{\mathbf{s}}_{n}-\overline{\mathbf{s}}_{n-1}\right]^{T} \nabla f_{n-1}\left(\overline{\mathbf{s}}_{n-1}\right)>0
$$

Dado que $0<f_{n} \leq 1$ e lembrando que o objetivo do algoritmo é maximizar essa função, então sua convergência estará assegurada se a propriedade de função convexa for satisfeita. Observando que $\nabla f_{n-1}\left(\overline{\mathbf{s}}_{n-1}\right)=\overline{\mathbf{s}}_{n-1}^{\odot 3}$ e substituindo esse resultado na desigualdade do lado direito de (2.88), chega-se a

$$
\overline{\mathbf{s}}_{n}^{T} \overline{\mathbf{s}}_{n-1}^{\odot 3}>\overline{\mathbf{s}}_{n-1}^{T} \overline{\mathbf{s}}_{n-1}^{\odot 3}
$$

Como $f_{n-1}=\overline{\mathbf{s}}_{n-1}^{T} \overline{\mathbf{s}}_{n-1}^{\odot 3}$, então

$$
\overline{\mathbf{s}}_{n}^{T} \overline{\mathbf{s}}_{n-1}^{\odot 3}>f_{n-1}
$$


Reescrevendo essa desigualdade em função do vetor $\mathbf{s}$ obtém-se

$$
\frac{\mathbf{s}_{n}^{T} \mathbf{s}_{n-1}^{\odot 3}}{\left\|\mathbf{s}_{n}\right\|\left\|\mathbf{s}_{n-1}\right\|^{3}}=\frac{\left\|\mathbf{s}_{n-1}\right\|}{\left\|\mathbf{s}_{n}\right\|} \frac{\mathbf{s}_{n}^{T} \mathbf{s}_{n-1}^{\odot 3}}{\left(\mathbf{s}_{n-1}^{T} \mathbf{s}_{n-1}\right)^{2}}>f_{n-1} .
$$

Elevando ambos os lados ao quadrado, chega-se a

$$
\frac{\left(\mathbf{s}_{n}^{T} \mathbf{s}_{n-1}^{\odot 3}\right)^{2}}{\left(\mathbf{s}_{n-1}^{T} \mathbf{s}_{n-1}\right)^{4}}>f_{n-1}^{2} \frac{\left\|\mathbf{s}_{n}\right\|^{2}}{\left\|\mathbf{s}_{n-1}\right\|^{2}} .
$$

Essa desigualdade permite obter os limites do passo de adaptação a partir das seguintes manipulações:

- Utilizando (2.83), o lado esquerdo de (2.92) pode ser reescrito como

$$
\begin{aligned}
\frac{\left(\mathbf{s}_{n}^{T} \mathbf{s}_{n-1}^{\odot 3}\right)^{2}}{\left(\mathbf{s}_{n-1}^{T} \mathbf{s}_{n-1}\right)^{4}} & =\left\{\frac{\left[\mathbf{s}_{n-1}^{T}+\mu_{n}\left(\mathbf{s}_{n-1}^{\odot 3 T}-\alpha_{n} \mathbf{s}_{n-1}^{T}\right)\right] \mathcal{P} \mathbf{s}_{n-1}^{\odot 3}}{\left(\mathbf{s}_{n-1}^{T} \mathbf{s}_{n-1}\right)^{2}}\right\}^{2} \\
& =\left\{f_{n-1}+\mu_{n} \frac{\mathbf{s}_{n-1}^{\odot 3 T} \mathcal{P} \mathbf{s}_{n-1}^{\odot 3}-\alpha_{n} \mathbf{s}_{n-1}^{T} \mathbf{s}_{n-1}^{\odot 3}}{\left(\mathbf{s}_{n-1}^{T} \mathbf{s}_{n-1}\right)^{2}}\right\}^{2} .
\end{aligned}
$$

- Calculando a norma ao quadrado de ambos os lados de (2.83), obtém-se

$$
\left\|\mathbf{s}_{n}\right\|^{2}=\left\|\mathbf{s}_{n-1}\right\|^{2}+\mu_{n}^{2}\left\|\mathcal{P}\left(\mathbf{s}_{n-1}^{\odot 3}-\alpha_{n} \mathbf{s}_{n-1}\right)\right\|^{2}+2 \mu_{n}\left(\mathbf{s}_{n-1}^{T} \mathbf{s}_{n-1}^{\odot 3}-\alpha_{n}\left\|\mathbf{s}_{n-1}\right\|^{2}\right),
$$

ou ainda

$$
\begin{aligned}
\left\|\mathbf{s}_{n}\right\|^{2}=\left\|\mathbf{s}_{n-1}\right\|^{2}+\mu_{n}^{2}\left(\left\|\mathcal{P} \mathbf{s}_{n-1}^{\odot 3}\right\|^{2}\right. & \left.+\alpha_{n}^{2}\left\|\mathbf{s}_{n-1}\right\|^{2}-2 \alpha_{n} \mathbf{s}_{n-1}^{T} \mathbf{s}_{n-1}^{\odot 3}\right) \\
& +2 \mu_{n}\left(\mathbf{s}_{n-1}^{T} \mathbf{s}_{n-1}^{\odot 3}-\alpha_{n}\left\|\mathbf{s}_{n-1}\right\|^{2}\right) .
\end{aligned}
$$

Dividindo ambos os lados de (2.95) por $\left\|\mathbf{s}_{n-1}\right\|^{2}$ e reconhecendo a igualdade $\mathbf{s}_{n-1}^{T} \mathbf{s}_{n-1}^{\odot 3} /\left\|\mathbf{s}_{n-1}\right\|^{2}=f_{n-1}\left\|\mathbf{s}_{n-1}\right\|^{2}$, chega-se $a$

$$
\frac{\left\|\mathbf{s}_{n}\right\|^{2}}{\left\|\mathbf{s}_{n-1}\right\|^{2}}=1+\mu_{n}^{2}\left(b_{n}+\alpha_{n}^{2}-2 \alpha_{n} f_{n-1}\left\|\mathbf{s}_{n-1}\right\|^{2}\right)+2 \mu_{n}\left(f_{n-1}\left\|\mathbf{s}_{n-1}\right\|^{2}-\alpha_{n}\right),
$$

sendo

$$
b_{n}=\frac{\left\|\mathcal{P}_{\mathbf{s}_{n-1}^{\odot 3}}^{\odot}\right\|^{2}}{\left\|\mathbf{s}_{n-1}\right\|^{2}} .
$$

Utilizando as equações (2.93) e (2.96), a Condição (2.92) pode ser reescrita como

$$
\begin{aligned}
{\left[f_{n-1}+\mu_{n}\left(b_{n}\left(\mathbf{s}_{n-1}^{T} \mathbf{s}_{n-1}\right)^{-1}-\alpha_{n} f_{n-1}\right)\right]^{2}>} & f_{n-1}^{2}\left[1+2 \mu_{n}\left(f_{n-1}\left\|\mathbf{s}_{n-1}\right\|^{2}-\alpha_{n}\right)\right. \\
& \left.+\mu_{n}^{2}\left(b_{n}-2 \alpha_{n} f_{n-1}\left\|\mathbf{s}_{n-1}\right\|^{2}+\alpha_{n}^{2}\right)\right] .
\end{aligned}
$$


Após algumas manipulações algébricas, obtém-se

$$
\mu_{n}<\frac{2 f_{n-1}}{2 \alpha_{n} f_{n-1}-\frac{b_{n}}{\mathbf{s}_{n-1}^{T} \mathbf{s}_{n-1}}} .
$$

Substituindo (2.97) em (2.99), chega-se à (2.86), o que completa a demonstração.

O intervalo do passo de adaptação que garante a convergência do algoritmo (2.77) pode ser interpretado como um caso particular do Teorema 1. Para isso, (2.84) e (2.85) devem ser consideradas em (2.86), o que leva a

$$
0<\mu_{n}<\frac{2 f_{n-1}}{2 f_{n-1}^{2}-\left\|\mathcal{P} \mathbf{s}_{n-1}^{\odot 3}\right\|^{2}} .
$$

Esse resultado foi obtido em [Mboup \& Regalia, 2000, Th.1].

\subsection{Algoritmos no domínio dos coeficientes do equali- zador}

O algoritmo Super-Exponencial e o algoritmo do Gradiente Projetado não-normalizado são descritos no domínio dos coeficientes do equalizador. Além disso, são estabelecidas duas outras formas de se chegar ao algoritmo de Shalvi-Weinstein baseado no gradiente determinístico (SD-SWA) da Equação (2.61, página 47). Os algoritmos são considerados no caso complexo.

Proposição 3 No domínio dos coeficientes do equalizador, o algoritmo Super-Exponencial da Equação (2.74) com $p=2$ é descrito pelas seguintes expressões

$$
\begin{aligned}
\widetilde{\mathbf{w}} & =\mathbf{R}^{-1} \mathbf{v}_{n}, \\
\mathbf{w}_{n} & =\sqrt{\frac{C_{1,1}^{a}}{\widetilde{\mathbf{w}}^{H} \mathbf{R} \widetilde{\mathbf{w}}}} \widetilde{\mathbf{w}},
\end{aligned}
$$

sendo

$$
\mathbf{v}_{n}=\frac{\mathrm{E}\left\{|y(n)|^{2} y(n) \mathbf{u}^{*}(n)\right\}-\beta C_{1,1}^{y} \mathrm{E}\left\{y(n) \mathbf{u}^{*}(n)\right\}}{C_{2,2}^{a}} .
$$

Demonstração: Lembrando que $\mathbf{s}_{n}=\mathcal{H}_{\mathbf{w}_{n}}$ e multiplicando ambos os lados dessa identidade por $\left(\mathcal{H}^{H} \mathcal{H}\right)^{-1} \mathcal{H}^{H}$, chega-se a

$$
\mathbf{w}_{n}=\left(\mathcal{H}^{H} \mathcal{H}\right)^{-1} \mathcal{H}^{H} \mathbf{s}_{n}
$$


Substituindo (2.74) com $p=2$ em (2.103) e notando que $\mathcal{H}^{H} \mathcal{P}=\mathcal{H}^{H}$, resulta em

$$
\mathbf{w}_{n}=\left(\mathcal{H}^{H} \mathcal{H}\right)^{-1} \frac{\mathcal{H}^{H} \mathbf{s}_{n-1}^{\odot<3>}}{\left\|\mathcal{P} \mathbf{s}_{n-1}^{\odot<3>}\right\|}
$$

Com o auxílio de (2.15), (2.21) e (2.24), obtém-se

$$
\mathbf{w}_{n}=C_{1,1}^{a} \mathbf{R}^{-1} \frac{\boldsymbol{C}_{2,1,1}^{y, y^{*}, \mathbf{u}^{*}}}{C_{2,2}^{a}}\left[C_{1,1}^{a} \frac{\boldsymbol{C}_{2,1,1}^{y^{*}, y, \mathbf{u}^{T}}}{C_{2,2}^{a}} \mathbf{R}^{-1} \frac{\boldsymbol{C}_{2,1,1}^{y, y^{*}, \mathbf{u}^{*}}}{C_{2,2}^{a}}\right]^{-1 / 2}
$$

Definindo

$$
\mathbf{v}_{n}=\frac{C_{2,1,1}^{y, y^{*} \mathbf{u}^{*}}}{C_{2,2}^{a}}
$$

a Equação (2.105) pode ser reescrita como

$$
\mathbf{w}_{n}=\frac{C_{1,1}^{a}}{\sqrt{C_{1,1}^{a}}} \frac{\mathbf{R}^{-1} \mathbf{v}_{n}}{\sqrt{\mathbf{v}_{n}^{H} \mathbf{R}^{-1} \mathbf{v}_{n}}} .
$$

Definindo ainda $\widetilde{\mathbf{w}}=\mathbf{R}^{-1} \mathbf{v}_{n}$ e utilizando (2.22) no cálculo de $\mathbf{v}_{n}$, obtêm-se as equações (2.101a) e (2.101b), o que completa a demonstração.

Proposição 4 O algoritmo do Gradiente Projetado não-normalizado da Equação (2.83) é descrito no domínio dos coeficientes do equalizador como

$$
\mathbf{w}_{n}=\mathbf{w}_{n-1}+\mu_{n} \frac{C_{1,1}^{a}}{C_{2,2}^{a}} \mathbf{R}^{-1}\left[\boldsymbol{C}_{2,1,1}^{y, y^{*} \mathbf{u}^{*}}-\alpha_{n} \frac{C_{2,2}^{a}}{C_{1,1}^{a}} \mathbf{R w}_{n-1}\right],
$$

ou ainda

$$
\mathbf{w}_{n}=\mathbf{w}_{n-1}-\mu_{n} \frac{\mathbf{R}^{-1}}{\gamma^{a}}\left[\mathrm{E}\left\{|y(n)|^{2} y(n) \mathbf{u}^{*}(n)\right\}-R_{2}^{a y} \mathrm{E}\left\{y(n) \mathbf{u}^{*}(n)\right\}\right]
$$

sendo

$$
R_{2}^{a y}=\beta C_{1,1}^{y}-\alpha_{n} \gamma^{a}
$$

Demonstração: Substituindo a identidade $\mathbf{s}_{n}=\mathcal{H} \mathbf{w}_{n}$ em (2.83), obtém-se

$$
\mathcal{H} \mathbf{w}_{n}=\mathcal{H} \mathbf{w}_{n-1}+\mu_{n} \underbrace{\mathcal{H}\left(\mathcal{H}^{H} \mathcal{H}\right)^{-1} \mathcal{H}^{H}}_{\mathcal{P}}\left(\mathbf{s}_{n-1}^{\odot<3>}-\alpha_{n} \mathcal{H} \mathbf{w}_{n-1}\right)
$$

Multiplicando ambos os lados de (2.111) por $\mathcal{H}^{H}$, chega-se a

$$
\mathcal{H}^{H} \mathcal{H} \mathbf{w}_{n}=\mathcal{H}^{H} \mathcal{H} \mathbf{w}_{n-1}+\mu_{n} \mathcal{H}^{H} \mathcal{H}\left(\mathcal{H}^{H} \mathcal{H}\right)^{-1}\left(\mathcal{H}^{H} \mathbf{s}_{n-1}^{\odot<3>}-\alpha_{n} \mathcal{H}^{H} \mathcal{H} \mathbf{w}_{n-1}\right)
$$


Utilizando (2.15) e (2.21), a Equação (2.112) pode ser reescrita como

$$
\frac{\mathbf{R}}{C_{1,1}^{a}} \mathbf{w}_{n}=\frac{\mathbf{R}}{C_{1,1}^{a}} \mathbf{w}_{n-1}+\mu_{n}\left[\frac{\boldsymbol{C}_{2,1,1}^{y, y^{*} \mathbf{u}^{*}}}{C_{2,2}^{a}}-\alpha_{n} \frac{\mathbf{R}}{C_{1,1}^{a}} \mathbf{w}_{n-1}\right] .
$$

Multiplicando ambos os lados de (2.113) por $C_{1,1}^{a} \mathbf{R}^{-1}$, obtém-se (2.108). Utilizando (2.22) $e(2.48)$ em (2.108) e notando que $\mathbf{R w}_{n-1}=\mathrm{E}\left\{y(n) \mathbf{u}^{*}(n)\right\}$, chega-se a (2.109) e (2.110), o que completa a demonstração.

Proposição 5 O algoritmo de Shalvi-Weinstein baseado no gradiente determinístico (SDSWA) de (2.61) pode ser obtido de duas formas distintas:

5.1) a partir do algoritmo (2.101) da Proposição 3, fazendo a normalização num ponto estacionário e assumindo o sinal de saída do equalizador com as mesmas estatísticas do sinal transmitido;

5.2) fazendo $C_{1,1}^{y}=C_{1,1}^{a}, \alpha_{n}=1$ e $\mu_{n}=1$ no algoritmo (2.109) da Proposição 4.

\section{Demonstração:}

5.1) Utilizando o gradiente de Shalvi-Weinstein da Tabela 2.1, página 44, define-se o gradiente de Shalvi-Weinstein modificado como

$$
\nabla_{\mathbf{w}} J_{S W M}(n)=\frac{\left(C_{1,1}^{y}\right)^{2}}{2(\beta-1)} \nabla_{\mathbf{w}} J_{S W}(n) .
$$

Cabe observar que os pontos estacionários obtidos a partir desses gradientes são idênticos porque $\boldsymbol{\nabla}_{\mathbf{w}} J_{S W}(n)=\mathbf{0}$ implica $\nabla_{\mathbf{w}} J_{S W M}(n)=\mathbf{0}$. Desta forma, considera-se por simplicidade o gradiente modificado. Reescrevendo o vetor $\mathbf{v}_{n}$ da Equação (2.102) em função $\boldsymbol{\nabla}_{\mathbf{w}} J_{S W M}(n)$ e utilizando (2.18, página 39), obtém

$$
\mathbf{v}_{n}=\frac{\nabla_{\mathbf{w}} J_{S W M}(n)-\gamma^{y} \mathbf{R} \mathbf{w}(n-1)}{C_{2,2}^{a}} .
$$

Num ponto estacionário $\nabla_{\mathbf{w}} J_{S W M}(n)=\mathbf{0}$ e a equação anterior se reduz a

$$
\mathbf{v}_{e}=-\frac{\gamma^{y} \mathbf{R} \mathbf{w}_{e}}{C_{2,2}^{a}}
$$

Substituindo (2.116) em (2.101a), obtém-se

$$
\widetilde{\mathbf{w}}_{e}=-\frac{\gamma^{y} \mathbf{w}_{e}}{C_{2,2}^{a}}
$$


e conseqüentemente o denominador de (2.101b) é calculado num ponto estacionário como

$$
\sqrt{\widetilde{\mathbf{w}}_{e}^{H} \mathbf{R} \widetilde{\mathbf{w}}_{e}}=\sqrt{\left(\frac{\gamma^{y}}{C_{2,2}^{a}}\right)^{2} \mathbf{w}_{e}^{H} \mathbf{R} \mathbf{w}_{e}}=\frac{\left|\gamma^{y}\right|}{\left|C_{2,2}^{a}\right|} \sqrt{C_{1,1}^{y}} .
$$

Combinando (2.101a) e (2.101b), fazendo $C_{1,1}^{y}=C_{1,1}^{a}$ e $R_{2}^{y}=R_{2}^{a}$ e considerando a normalização num ponto estacionário da Equação (2.118), obtém-se a seguinte atualização dos coeficientes

$$
\mathbf{w}(n)=\frac{\left|C_{2,2}^{a}\right|}{C_{2,2}^{a}}\left[-\frac{\gamma^{a}}{\left|\gamma^{a}\right|} \mathbf{w}(n-1)+\frac{\left.\mathbf{R}^{-1} \nabla_{\mathbf{w}} J_{S W M}(n)\right|_{R_{2}^{y}=R_{2}^{a}}}{\left|\gamma^{a}\right|}\right] .
$$

Considerando que o sinal transmitido tenha curtose negativa, então $\gamma^{a}=\left|\gamma^{a}\right|$ e

$$
-\frac{\gamma^{a}}{\left|\gamma^{a}\right|}=\frac{\left|C_{2,2}^{a}\right|}{C_{2,2}^{a}}=-1
$$

e a Equação (2.119) se reduz à (2.61) o que completa a primeira parte da demonstração.

5.2) Se $C_{1,1}^{y}=C_{1,1}^{a}, \alpha_{n}=1$ e $\mu_{n}=1$, então

$$
R_{2}^{a y}=\frac{\mathrm{E}\left\{|a(n)|^{4}\right\}}{\mathrm{E}\left\{|a(n)|^{2}\right\}}=R_{2}^{a}
$$

Substituindo $R_{2}^{a y}$ em (2.109) chega-se a (2.61) o que completa a demonstração.

A partir das proposições 3, 4 e 5 as seguintes observações podem ser feitas:

- o algoritmo da Proposição 3 foi obtido em [Shalvi \& Weinstein, 1990, 1993, 1994];

- o algoritmo do Gradiente Projetado não-normalizado da Proposição 4 resulta no algoritmo Super-Exponencial da Proposição 3, considerando (2.24) no cálculo da norma e fazendo

$$
\alpha_{n}=1 / \mu_{n}=\left\|\mathcal{P} \mathbf{s}_{n-1}^{\odot<3>}\right\|
$$

- o SD-SWA pode ser interpretado como um algoritmo Super-Exponencial simplificado. Cabe observar que tanto na Proposição 5 quanto na Proposição 2, utiliza-se efetivamente a função custo de Godard e não a de Shalvi-Weinstein, dado que se consideram as estatísticas do sinal transmitido;

- o SD-SWA também pode ser deduzido a partir da minimização da função custo de Shalvi-Weinstein, considerando cumulantes empíricos como foi feito em [Shalvi \& Weinstein, 1993, 1994]. 


\subsection{A convergência do SD-CMA}

Inspirando-se no Teorema 1 da Seção 2.5, é apresentado a seguir um intervalo do passo de adaptação que garante a convergência do SD-CMA nos domínios da resposta combinada e dos coeficientes do equalizador. A análise é feita para sinais reais mas pode ser estendida para o caso complexo.

Teorema 2 O algoritmo do Módulo Constante baseado no gradiente determinístico (SDCMA) de (2.58) pode ser reescrito no dominio da resposta combinada como

$$
\mathbf{s}_{n}=\mathbf{s}_{n-1}+\tilde{\mu}_{n} \mathcal{H} \mathcal{H}^{T} \mathbf{x}_{n-1}
$$

sendo

$$
\begin{aligned}
& \tilde{\mu}_{n}=-\mu_{n} C_{4}^{a}, \\
& \mathbf{x}_{n}=\mathbf{s}_{n}^{\odot 3}-\tilde{\alpha}_{n} \mathbf{s}_{n},
\end{aligned}
$$

$e$

$$
\tilde{\alpha}_{n}=-\frac{\left(3 C_{2}^{a}\left\|\mathbf{s}_{n}\right\|^{2}-R_{2}^{a}\right) C_{2}^{a}}{C_{4}^{a}} .
$$

Esse algoritmo terá convergência assegurada se, para cada iteração n, o passo de adaptação $\tilde{\mu}_{n}$ estiver no intervalo

$$
0<\tilde{\mu}_{n}<\frac{2 f_{n-1}}{\mathbf{s}_{n-1}^{T} \mathbf{s}_{n-1}}\left[\frac{\mathbf{z}_{n-1}^{T} \mathcal{H} \mathcal{H}^{T} \mathbf{x}_{n-1}}{f_{n-1}^{2} \mathbf{x}_{n-1}^{T} \mathcal{H} \mathcal{H}^{T} \mathcal{H} \mathcal{H}^{T} \mathbf{x}_{n-1}-\left(\mathbf{s}_{n-1}^{T} \mathbf{s}_{n-1}\right)^{-3}\left(\mathbf{s}_{n-1}^{\odot 3 T} \mathcal{H} \mathcal{H}^{T} \mathbf{x}_{n-1}\right)^{2}}\right]
$$

sendo

$$
\mathbf{z}_{n}=\mathbf{s}_{n}^{\odot 3}-\left(f_{n} \mathbf{s}_{n}^{T} \mathbf{s}_{n}\right) \mathbf{s}_{n}
$$

Demonstração: Utilizando as relações (2.22) e (2.18) da Seção 2.1, a atualização do SD-CMA (Equação (2.58)) pode ser reescrita em função de cumulantes, ou seja,

$$
\mathbf{w}_{n}=\mathbf{w}_{n-1}-\mu_{n}\left[\boldsymbol{C}\left(y_{n}^{3}, \mathbf{u}_{n}\right)+\left(3 C_{2}^{y}-R_{2}^{a}\right) \mathbf{R w}_{n-1}\right] .
$$

Multiplicando ambos os lados dessa equação por $\mathcal{H}$, lembrando que $\mathcal{H} \mathbf{w}_{n}=\mathbf{s}_{n}$ e utilizando as relações (2.21), (2.17) e (2.18), chega-se a

$$
\mathbf{s}_{n}=\mathbf{s}_{n-1}-\mu_{n} \mathcal{H}\left[C_{4}^{a} \mathcal{H}^{T} \mathbf{s}_{n-1}^{\odot 3}+\left(3 C_{2}^{a} \mathbf{s}_{n-1}^{T} \mathbf{s}_{n-1}-R_{2}^{a}\right) C_{2}^{a} \mathcal{H}^{T} \mathbf{s}_{n-1}\right]
$$


Através das definições (2.121) e (2.123), a Equação (2.127) se reduz a

$$
\mathbf{s}_{n}=\mathbf{s}_{n-1}-\tilde{\mu}_{n} \mathcal{H} \mathcal{H}^{T}\left[\mathbf{s}_{n-1}^{\odot 3}-\tilde{\alpha}_{n-1} \mathbf{s}_{n-1}\right]
$$

Utilizando (2.122), obtém-se (2.120) que é a equação do SD-CMA no domínio da resposta combinada. Calculando a norma ao quadrado de ambos os lados de (2.120), chega-se a

$$
\left\|\mathbf{s}_{n}\right\|^{2}=\left\|\mathbf{s}_{n-1}\right\|^{2}+2 \tilde{\mu}_{n} \mathbf{s}_{n-1}^{T} \boldsymbol{H} \mathcal{H}^{T} \mathbf{x}_{n-1}+\tilde{\mu}_{n}^{2} \mathbf{x}_{n-1}^{T} \mathcal{H} \mathcal{H}^{T} \mathcal{H} \mathcal{H}^{T} \mathbf{x}_{n-1}
$$

Observando que

$$
\frac{\mathbf{s}_{n}^{T} \mathbf{s}_{n-1}^{\odot 3}}{\left(\mathbf{s}_{n-1}^{T} \mathbf{s}_{n-1}\right)^{2}}=\frac{\left(\mathbf{s}_{n-1}^{T}+\tilde{\mu}_{n} \mathbf{x}_{n-1} \mathcal{H} \mathcal{H}^{T}\right) \mathbf{s}_{n-1}^{\odot 3}}{\left(\mathbf{s}_{n-1}^{T} \mathbf{s}_{n-1}\right)^{2}}=f_{n-1}+\tilde{\mu}_{n} \frac{\mathbf{x}_{n-1}^{T} \mathcal{H} \mathcal{H}^{T} \mathbf{s}_{n-1}^{\odot 3}}{\left(\mathbf{s}_{n-1}^{T} \mathbf{s}_{n-1}\right)^{2}}
$$

e aplicando a condição de função convexa de (2.92, página 55), obtém-se

$$
\begin{aligned}
{\left[f_{n-1}+\tilde{\mu}_{n} \frac{\mathbf{x}_{n-1}^{T} \mathcal{H} \mathcal{H}^{T} \mathbf{s}_{n-1}^{\odot 3}}{\left(\mathbf{s}_{n-1}^{T} \mathbf{s}_{n-1}\right)^{2}}\right]^{2}>f_{n-1}^{2} } & {\left[1+2 \tilde{\mu}_{n} \frac{\mathbf{s}_{n-1}^{T} \mathcal{H H}^{T} \mathbf{x}_{n-1}}{\mathbf{s}_{n-1}^{T} \mathbf{s}_{n-1}}\right.} \\
& \left.+\tilde{\mu}_{n}^{2} \frac{\mathbf{x}_{n-1}^{T} \boldsymbol{\mathcal { H }} \mathcal{H}^{T} \boldsymbol{H} \mathcal{H}^{T} \mathbf{x}_{n-1}}{\mathbf{s}_{n-1}^{T} \mathbf{s}_{n-1}}\right] .
\end{aligned}
$$

Desenvolvendo o quadrado do lado esquerdo dessa expressão e fazendo algumas manipulações algébricas, chega-se a

$$
\begin{aligned}
2 f_{n-1} \frac{\mathbf{x}_{n-1}^{T} \mathcal{H} \mathcal{H}^{T} \mathbf{s}_{n-1}^{\odot 3}}{\left(\mathbf{s}_{n-1}^{T} \mathbf{s}_{n-1}\right)^{2}}+\tilde{\mu}_{n}\left[\frac{\mathbf{x}_{n-1}^{T} \mathcal{H} \mathcal{H}^{T} \mathbf{s}_{n-1}^{\odot 3}}{\left(\mathbf{s}_{n-1}^{T} \mathbf{s}_{n-1}\right)^{2}}\right]^{2}> & 2 f_{n-1}^{2} \frac{\mathbf{s}_{n-1}^{T} \mathcal{H}^{T} \mathbf{H}_{n-1}}{\mathbf{s}_{n-1}^{T} \mathbf{s}_{n-1}} \\
& +\tilde{\mu}_{n} f_{n-1}^{2} \frac{\mathbf{x}_{n-1}^{T} \mathcal{H} \mathcal{H}^{T} \mathcal{H H}^{T} \mathbf{x}_{n-1}}{\mathbf{s}_{n-1}^{T} \mathbf{s}_{n-1}}
\end{aligned}
$$

ou ainda

$$
\begin{aligned}
\tilde{\mu}_{n}\left[f_{n-1}^{2} \frac{\mathbf{x}_{n-1}^{T} \mathcal{H} \mathcal{H}^{T} \mathcal{H} \mathcal{H}^{T} \mathbf{x}_{n-1}}{\mathbf{s}_{n-1}^{T} \mathbf{s}_{n-1}}\right. & \left.-\frac{\left(\mathbf{x}_{n-1}^{T} \mathcal{H} \mathcal{H}^{T} \mathbf{s}_{n-1}^{\odot 3}\right)^{2}}{\left(\mathbf{s}_{n-1}^{T} \mathbf{s}_{n-1}\right)^{4}}\right] \\
& <\frac{2 f_{n-1}}{\mathbf{s}_{n-1}^{T} \mathbf{s}_{n-1}} \mathbf{x}_{n-1}^{T} \mathcal{H} \mathcal{H}^{T}\left[\frac{\mathbf{s}_{n-1}^{\odot 3}}{\mathbf{s}_{n-1}^{T} \mathbf{s}_{n-1}}-f_{n-1} \mathbf{s}_{n-1}\right] .
\end{aligned}
$$

Utilizando (2.125), obtém-se

$$
\tilde{\mu}_{n}\left[f_{n-1}^{2} \mathbf{x}_{n-1}^{T} \mathcal{H} \mathcal{H}^{T} \mathcal{H} \mathcal{H}^{T} \mathbf{x}_{n-1}-\frac{\left(\mathbf{x}_{n-1}^{T} \mathcal{H} \mathcal{H}^{T} \mathbf{s}_{n-1}^{\odot 3}\right)^{2}}{\left(\mathbf{s}_{n-1}^{T} \mathbf{s}_{n-1}\right)^{3}}\right]<\frac{2 f_{n-1}}{\mathbf{s}_{n-1}^{T} \mathbf{s}_{n-1}} \mathbf{x}_{n-1}^{T} \mathcal{H} \mathcal{H}^{T} \mathbf{z}_{n-1}
$$

A partir dessa expressão, obtém-se (2.124) o que completa a demonstração. 
Se a matriz de autocorrelação do sinal de entrada tiver a forma $\mathbf{R}=C_{2}^{a} \mathbf{I}$, o operador de projeção se reduzirá a $\mathcal{P}=\mathcal{H} \mathcal{H}^{T}$. Isso pode ser obtido através do pré-branqueamento do sinal $u(n)$. Além disso, se o parâmetro $\alpha_{n}$ de (2.83, página 53) for igual a $\tilde{\alpha}_{n}$ de (2.123), os intervalos do passo de adaptação do Teorema 2 e do Teorema 1 coincidem.

O intervalo do passo de adaptação do Teorema 2 pode ser escrito no domínio dos coeficientes do equalizador como é mostrado na Proposição 6 a seguir.

Proposição 6 Assumindo que o sinal transmitido possui curtose negativa, definindo

$$
e^{y}(n)=\left(|y(n)|^{2}-R_{2}^{y}\right) y(n)
$$

e utilizando as definições de e(n) e $\gamma^{y}$ das equações (2.59) e (2.49) respectivamente, o intervalo do passo de adaptação de (2.124) pode ser escrito no domínio dos coeficientes do equalizador como

$$
0<\mu_{n}<\frac{2 C_{4}^{y} \mathrm{E}\left\{e^{y} \mathbf{u}^{T}\right\} \mathrm{E}\{e \mathbf{u}\}}{\left[\left(\mathrm{E}\left\{e^{y} \mathbf{u}^{T}\right\}+\gamma^{y} \mathrm{E}\left\{y \mathbf{u}^{T}\right\}\right) \mathrm{E}\{e \mathbf{u}\}\right]^{2}-\left(C_{4}^{y}\right)^{2}\left(C_{2}^{y}\right)^{-1} \mathrm{E}\left\{e \mathbf{u}^{T}\right\} \mathbf{R E}\{e \mathbf{u}\}}
$$

No lado direito dessa expressão os índices de tempo foram suprimidos para compactar a notação.

Demonstração: Multiplicando $\mathbf{z}_{n-1}$ definido em (2.125) por $\mathcal{H}^{T}$, obtém-se

$$
\mathcal{H}^{T} \mathbf{z}_{n-1}=\mathcal{H}^{T} \mathbf{s}_{n-1}^{\odot 3}-\left(f_{n-1} \mathbf{s}_{n-1}^{T} \mathbf{s}_{n-1}\right) \mathcal{H}^{T} \mathbf{s}_{n-1}
$$

Utilizando as relações (2.18) e (2.21) da Seção 2.1, pode-se reescrever (2.137) como

$$
\mathcal{H}^{T} \mathbf{z}_{n-1}=\frac{\boldsymbol{C}\left(y_{n}^{3}, \mathbf{u}_{n}\right)}{C_{4}^{a}}-\left(f_{n-1} \mathbf{s}_{n-1}^{T} \mathbf{s}_{n-1}\right) \frac{\mathrm{E}\left\{y_{n} \mathbf{u}_{n}\right\}}{C_{2}^{a}}
$$

Com o auxílio das definições de $f_{n-1}$ e de $\gamma^{y}$ dadas respectivamente em (2.76, página 52) e (2.49, página 45) e utilizando as relações (2.17) e (2.19), chega-se a

$$
\mathcal{H}^{T} \mathbf{z}_{n-1}=\frac{1}{C_{4}^{a}}\left(\boldsymbol{C}\left(y_{n}^{3}, \mathbf{u}_{n}\right)+\gamma^{y} \mathrm{E}\left\{y_{n} \mathbf{u}_{n}\right\}\right)
$$

Utilizando a relação (2.22) e a definição de e $e^{y}(n)$ de (2.135), obtém-se finalmente

$$
\mathcal{H}^{T} \mathbf{z}_{n-1}=\frac{1}{C_{4}^{a}} \mathrm{E}\left\{e_{n}^{y} \mathbf{u}_{n}\right\}
$$


Analogamente, as seguintes relações podem ser obtidas

$$
\mathcal{H}^{T} \mathbf{x}_{n-1}=\frac{1}{C_{4}^{a}} \mathrm{E}\left\{e_{n} \mathbf{u}_{n}\right\}
$$

$e$

$$
\mathcal{H}^{T} \mathbf{s}_{n-1}^{\odot 3}=\frac{1}{C_{4}^{a}}\left(\mathrm{E}\left\{e_{n}^{y} \mathbf{u}_{n}\right\}+\gamma^{y} \mathrm{E}\left\{y_{n} \mathbf{u}_{n}\right\}\right) .
$$

Substituindo (2.140), (2.141), (2.142) e (2.121) em (2.124) e utilizando as relações (2.17) e (2.19), após algumas manipulações algébricas, obtém-se (2.136), o que completa a demonstração.

\subsection{Resultados experimentais}

Nesta seção, é verificado o comportamento dos algoritmos autodidatas do gradiente determinístico SD-CMA e SD-SWA das equações (2.58) e (2.61) em relação ao algoritmo SuperExponencial de (2.101). Além disso, são mostrados resultados para validar as condições para convergência do SD-CMA.

O canal de fase não-mínima utilizado tem a seguinte função de transferência [Shalvi \& Weinstein, 1994]

$$
\mathrm{H}_{2}(z)=0,4+z^{-1}-0,7 z^{-2}+0,6 z^{-3}+0,3 z^{-4}-0,4 z^{-5}+0,1 z^{-6} .
$$

Assume-se um equalizador com $M=16$ coeficientes inicializados da forma

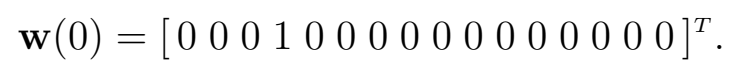

Além disso, como medida de desempenho considera-se uma média de 200 realizações da interferência intersimbólica (ISI) residual, definida na Equação (2.4, página 34).

As estimativas do vetor gradiente, matriz de autocorrelação e outras variáveis que dependem de $y(n)$ são feitas com blocos de dados de $N_{b}$ amostras. Por exemplo, para estimar $\mathrm{E}\left\{|y(n)|^{2}\right\}$ em cada instante de tempo $n$, o vetor de coeficientes é mantido fixo em $\mathbf{w}(n-1)$ e a partir de $N_{b}$ vetores de entrada $\mathbf{u}$, calculam-se $N_{b}$ saídas do equalizador. Utilizando essas saídas, faz-se a estimativa desejada. Depois de estimar o vetor gradiente, os coeficientes são atualizados e uma nova iteração se inicia. Quanto maior o comprimento do bloco de dados, melhor é a qualidade das estimativas. 
Na Figura 2.3, são mostradas curvas de ISI residual média dos algoritmos Super-Exponencial (SEA), do Módulo Constante (SD-CMA) e de Shalvi-Weinstein (SD-SWA), baseados no gradiente determinístico. São utilizados três comprimentos diferentes para os blocos de dados, ou seja, $N_{b}=420, N_{b}=600$ e $N_{b}=4800$. Pode-se observar na Figura 2.3-c que, na média, o SEA possui uma convergência quase instantânea (apenas 4 iterações) seguido do SD-SWA (em torno de 80 iterações) e do SD-CMA (aproximadamente 720 iterações). Embora a convergência do SEA seja atrativa, ele sofre mais com a qualidade das estimativas que os outros algoritmos. Diminuindo o comprimento do bloco para $N_{b}=420$ (Figura 2.3-a), observa-se que enquanto o SD-CMA e o SD-SWA recuperam a seqüência transmitida, o SEA fica "encalhado" num mínimo local, não equalizando efetivamente o canal. Esse comportamento se repete para canais e modulações diferentes. Em contrapartida, embora o SD-CMA seja o mais lento dentre os algoritmos considerados, ele possui a menor complexidade computacional, dado que seu passo de adaptação é um escalar e não uma matriz.
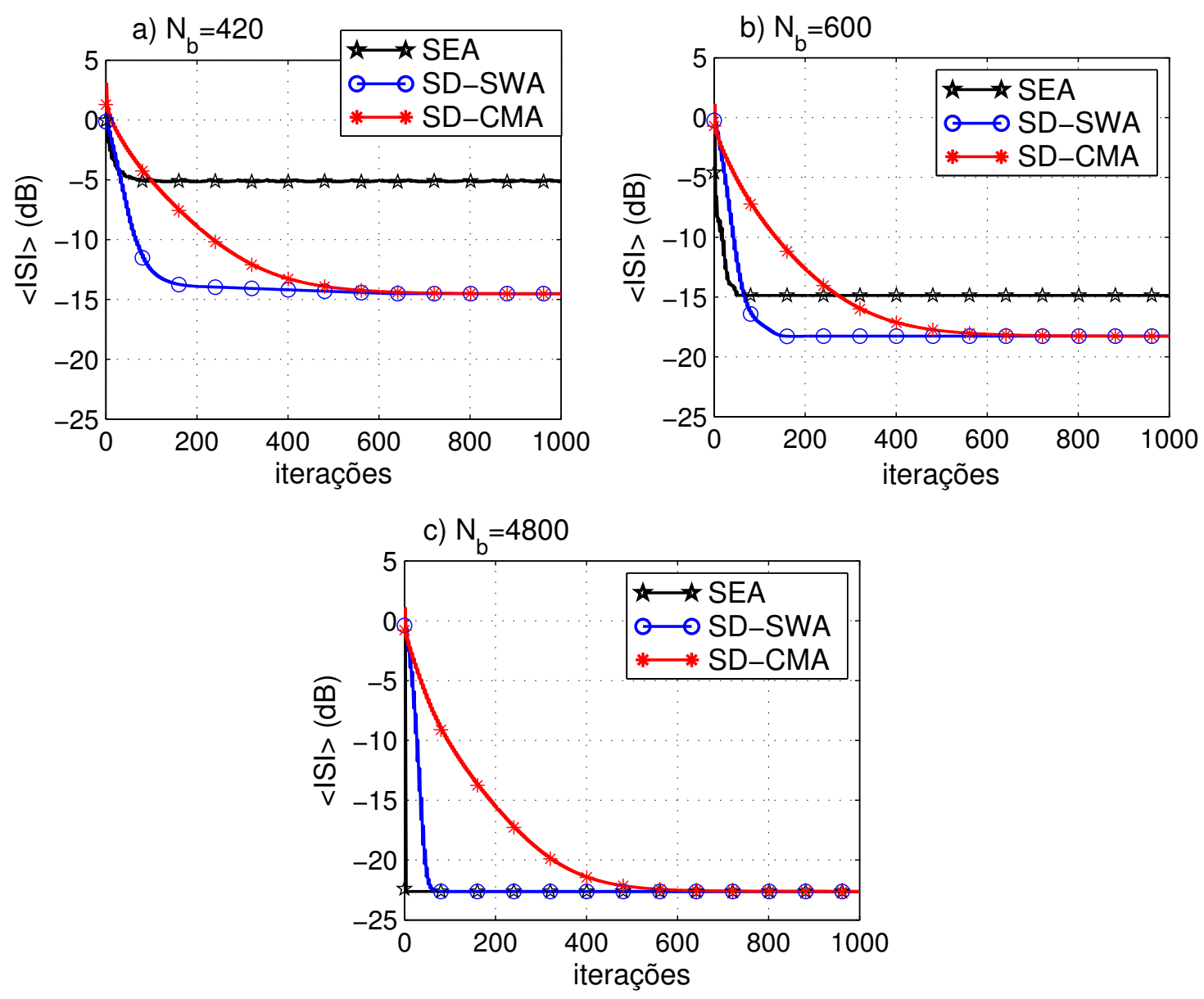

Figura 2.3: ISI residual média (200 realizações) em função de $N_{b}$ do $\operatorname{SEA}, \operatorname{SD}-\operatorname{SWA}\left(\beta_{S W}=0,1\right)$ e SD-CMA $\left(\mu=10^{-4}\right) ; 6$-PAM, canal $\mathrm{H}_{2}$ e $M=16$. 
Segundo o Teorema 2, a convergência do SD-CMA será assegurada se a cada instante de tempo, o passo de adaptação $\mu_{n}$ estiver no intervalo

$$
0<\mu_{n}<\mu_{n_{\max }}
$$

sendo $\mu_{n_{\max }}$ o limite máximo do intervalo de (2.136). Considerando uma margem de segurança, ou seja, fazendo $\mu_{n}=0,1 \mu_{n \max }$ a cada iteração e variando o valor de $N_{b}$, obtêm-se os resultados das figuras 2.4, 2.5 e 2.6. Na Figura 2.4, são mostradas curvas de ISI residual média. Observa-se que com a escolha de $\mu_{n}=0,1 \mu_{n \max }$, o SD-CMA converge independentemente da qualidade das estimativas. Embora seu desempenho piore à medida que o tamanho do bloco de dados diminui, ele não diverge. Na Figura 2.5, é mostrada a média do passo de adaptação para os valores de $N_{b}$ considerados. É interessante observar que depois da convergência inicial, o passo de adaptação vai diminuindo e tende para um valor constante. Além disso, quanto maior $N_{b}$, mais rápido é o decaimento. Na Figura 2.6, é mostrada a média dos coeficientes ao longo das iterações. À medida que o valor de $N_{b}$ aumenta, os coeficientes apresentam uma variação menor em torno do patamar de convergência.

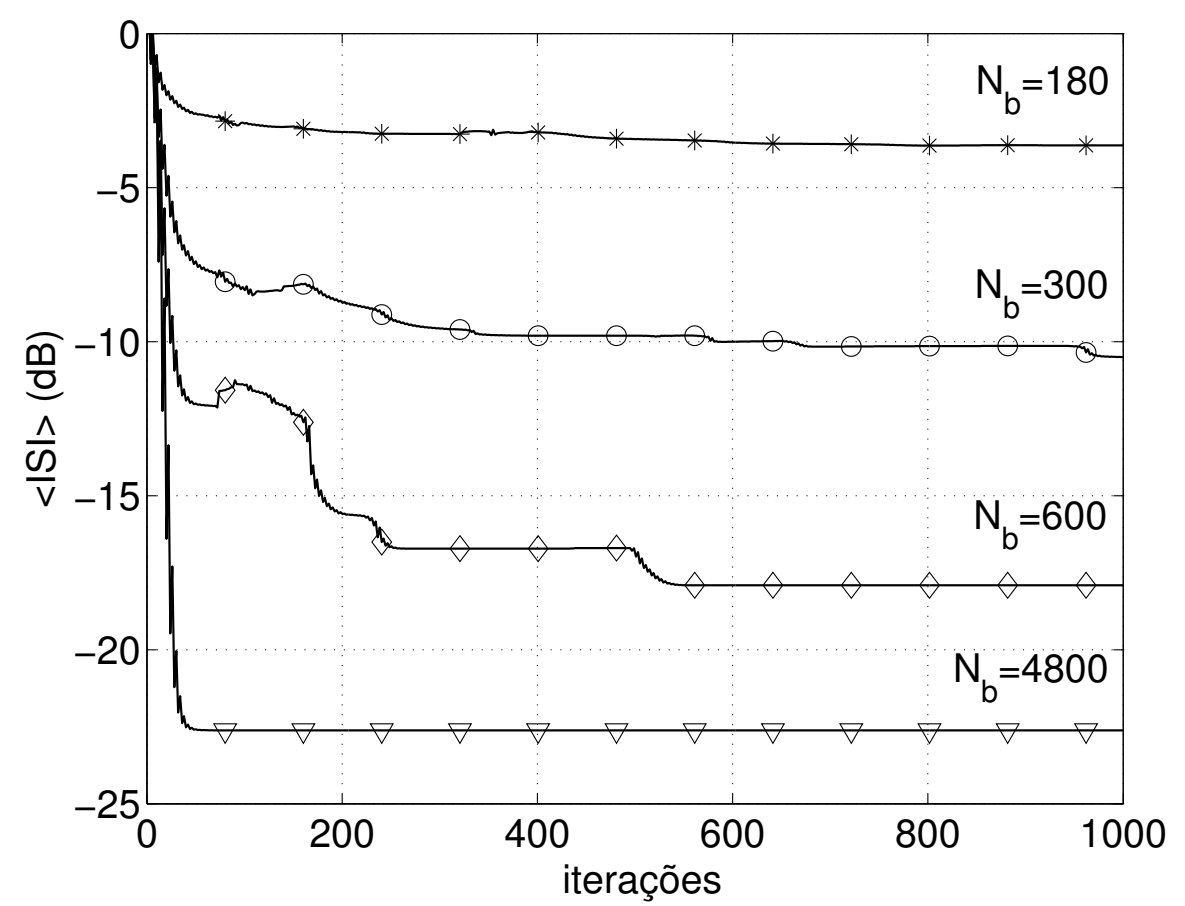

Figura 2.4: ISI residual média (200 realizações) do SD-CMA para diferentes valores de $N_{b} ; \mu_{n}=$ $0,1 \mu_{n \max }, 6$-PAM, canal $\mathrm{H}_{2}$ e $M=16$. 

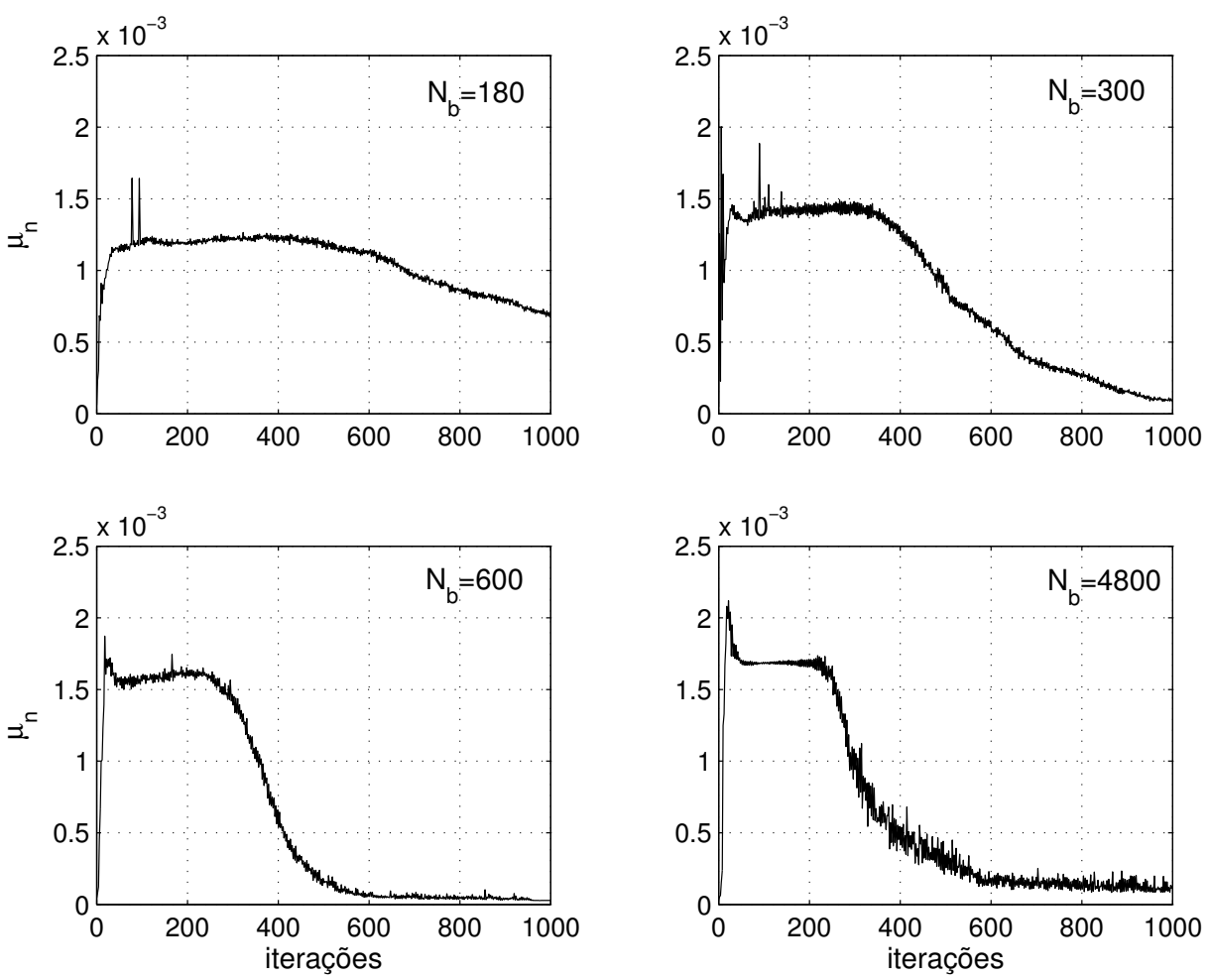

Figura 2.5: Passo de adaptação médio (200 realizações) do SD-CMA para diferentes valores de $N_{b} ; \mu_{n}=0,1 \mu_{n_{\max }}, 6$-PAM, canal $\mathrm{H}_{2}$ e $M=16$.
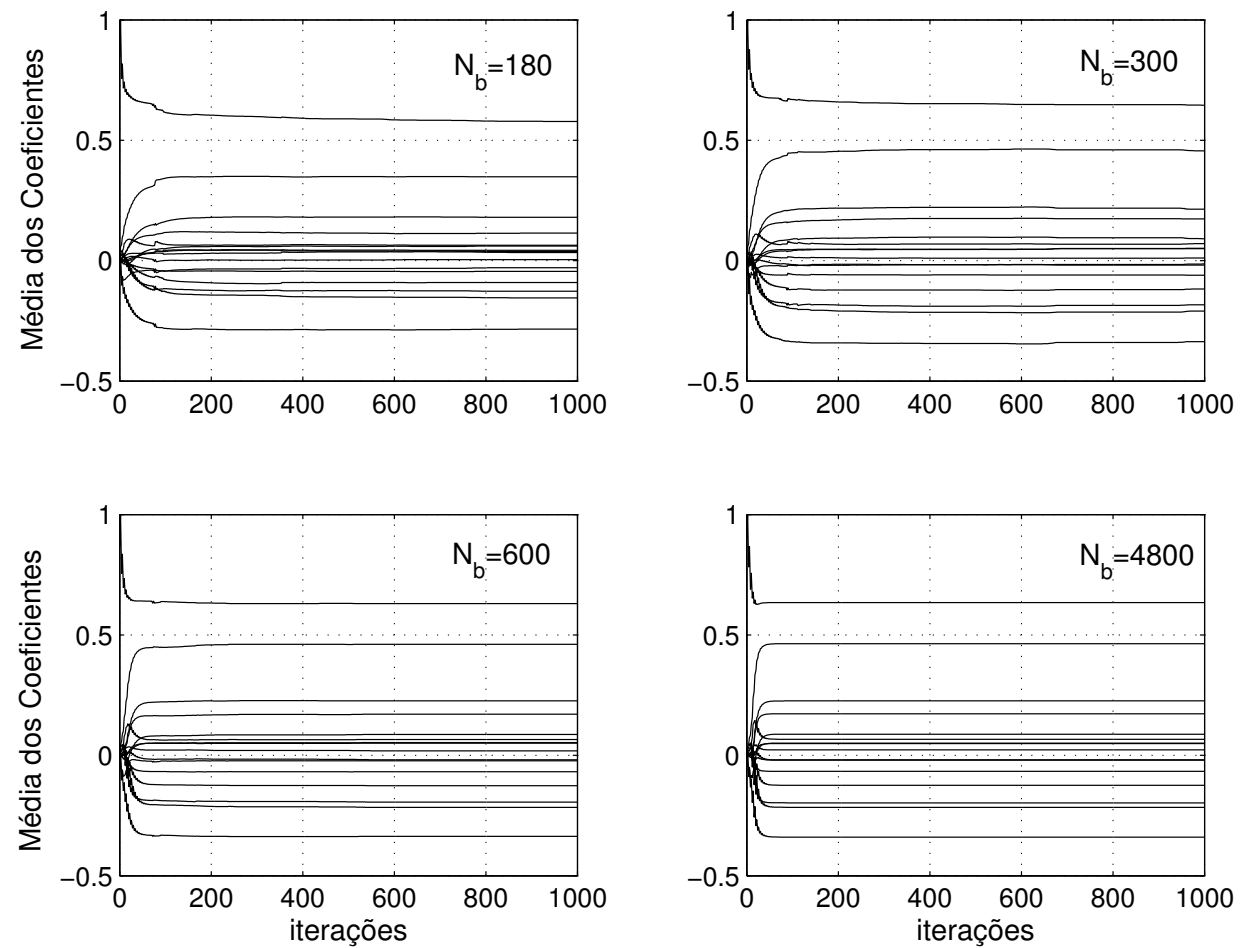

Figura 2.6: Média (200 realizações) dos coeficientes do SD-CMA para diferentes valores de $N_{b}$; $\mu_{n}=0,1 \mu_{n_{\max }}, 6$-PAM, canal $\mathrm{H}_{2}$ e $M=16$. 
Na Figura 2.7, são mostradas curvas de ISI residual média, considerando-se o tamanho do bloco fixo $\left(N_{b}=600\right)$ e variando-se a margem de segurança do limite máximo $\mu_{n_{\max }}$. Pode-se observar que com $\mu_{n}=0,1 \mu_{n_{\max }}$ ou $\mu_{n}=0,5 \mu_{n \max }$, o algoritmo converge na média para o mesmo patamar de ISI, sendo que com $\mu_{n}=0,1 \mu_{n_{\max }}$, ele apresenta uma velocidade de convergência média maior. No entanto, para $\mu_{n}=0,01 \mu_{n_{\max }}$, a convergência se torna mais lenta. No caso limite em que $\mu_{n}=\mu_{n_{\max }}$, o SD-CMA não diverge, porém, seu comportamento na média não é satisfatório, já que em algumas realizações ele consegue convergir para uma boa solução e em outras isso não ocorre. Desta forma, conclui-se que há uma margem de segurança ótima, que, neste caso, deve ser escolhida no intervalo $] 0,01 ; 0,5[$. Na Figura 2.8, são mostradas as médias dos coeficientes e dos passos de adaptação ao longo das iterações. Cabe notar que o passo de adaptação tende para um valor constante. No caso de $\mu_{n}=0,01 \mu_{n_{\max }}$, ele parte de um valor bem pequeno e vai aumentando ao longo das iterações, comportamento contrário ao dos outros casos. Além disso, quanto maior a margem de segurança, menor é a variância do passo de adaptação. Essa variância pode afetar a convergência inicial, como pode ser observado na média dos coeficientes para o caso de $\mu_{n}=0,5 \mu_{n \max }$. Esses resultados também foram observados com outros canais e outras modulações.

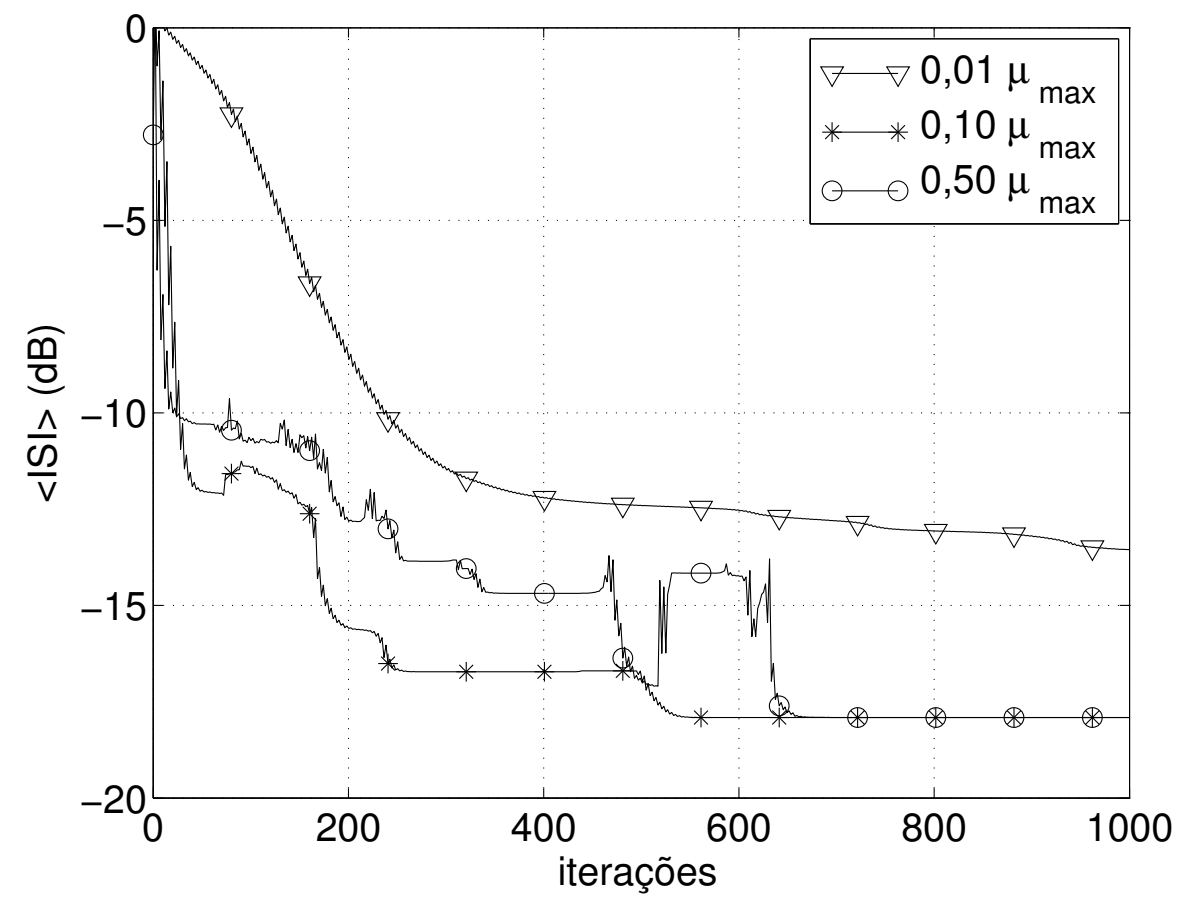

Figura 2.7: ISI residual média (200 realizações) do SD-CMA em função de $\mu_{n} ; N_{b}=600,6$-PAM, canal $\mathrm{H}_{2}$ e $M=16$. 

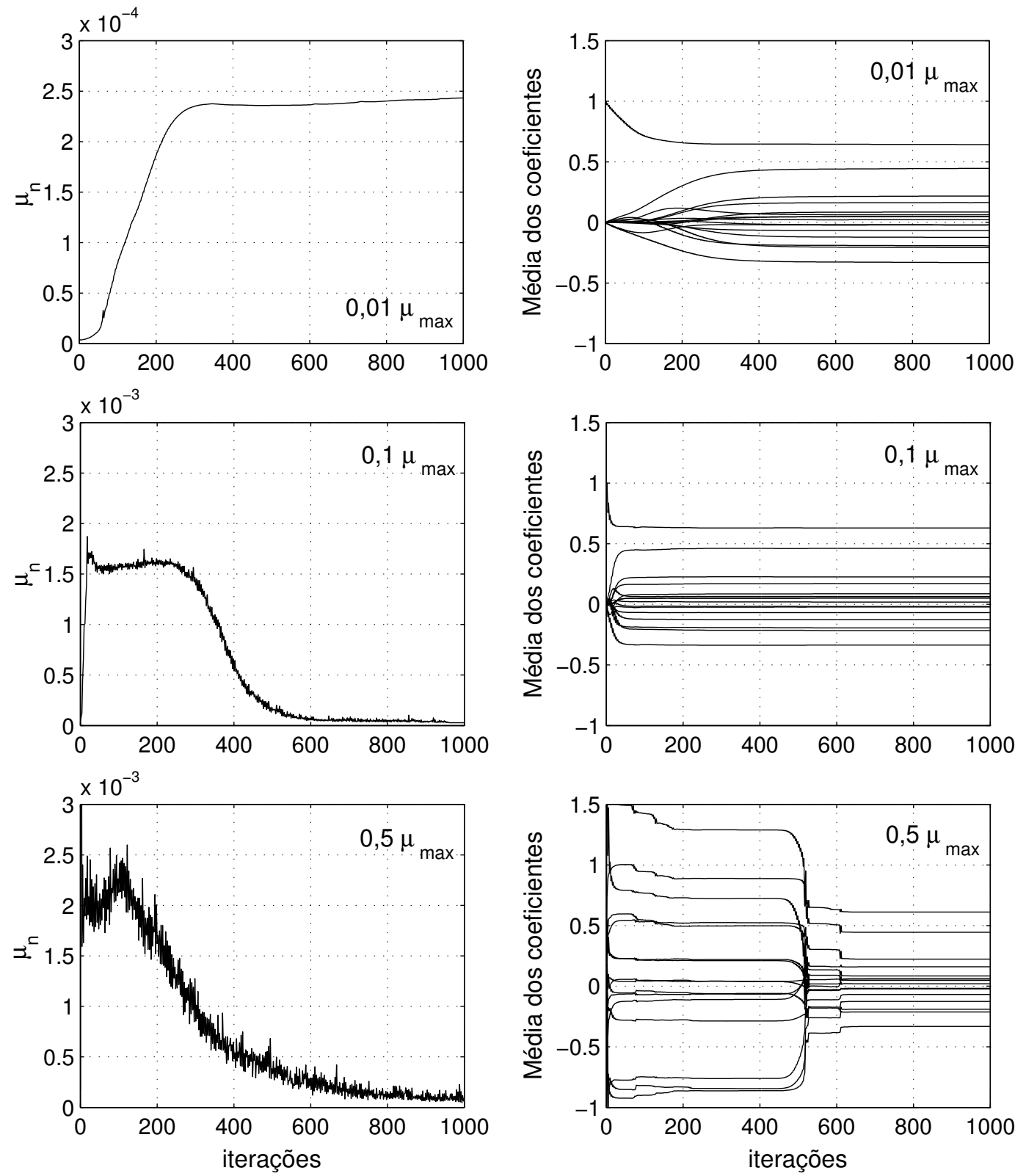

Figura 2.8: Média (200 realizações) dos coeficientes e do passo de adaptação do SD-CMA em função de $\mu_{n} ; N_{b}=600$, 6-PAM, canal $\mathrm{H}_{2}$ e $M=16$.

\subsection{Conclusões}

Neste capítulo, os algoritmos do Módulo Constante (SD-CMA) e de Shalvi-Weinstein (SDSWA) baseados no gradiente determinístico foram abordados. A equivalência entre os critérios do Módulo Constante e de Shalvi-Weinstein, baseada na igualdade de potências das saídas dos equalizadores, foi obtida em [Regalia, 1999] e é um resultado consolidado na literatura. Além disso, a interpretação do algoritmo de Shalvi-Weinstein como um algoritmo 
do Gradiente Projetado e sua análise de convergência decorrem dos trabalhos de Mboup e Regalia (2000). Neste contexto, as principais contribuições deste capítulo estão nas relações entre os algoritmos e na extensão da análise de convergência de [Mboup \& Regalia, 2000] para o algoritmo do Módulo Constante do gradiente determinístico.

Na Tabela 2.2, é mostrado um resumo dos algoritmos e análises de convergência deste capítulo. São identificados os números das equações, as proposições e os teoremas relacionados. Além disso, faz-se referência aos trabalhos em que os resultados já conhecidos foram apresentados.

\begin{tabular}{|c|c|c|c|}
\hline Algoritmo & $\begin{array}{c}\text { Domínio da resposta } \\
\text { combinada }\end{array}$ & $\begin{array}{c}\text { Domínio dos coeficientes } \\
\text { do equalizador }\end{array}$ & $\begin{array}{c}\text { Intervalo do passo } \\
\text { de adaptação }\end{array}$ \\
\hline SD-CMA & $\begin{array}{c}\text { Teorema } 2 \\
(2.120)\end{array}$ & $\begin{array}{c}(2.58) \\
{[\text { Godard, 1980] }} \\
{[\text { Treichler \& Agee, 1983] }}\end{array}$ & $\begin{array}{c}\text { Teorema } 2 \text { e } \\
\text { Proposição } 6 \\
(2.124) \text { e }(2.136)\end{array}$ \\
\hline SD-SWA & - & $\begin{array}{c}\text { Proposições } 2 \text { e } 5 \\
(2.61) \\
\text { [Shalvi \& Weinstein, } \\
1990,1993]\end{array}$ & - \\
\hline $\begin{array}{c}\text { Super- } \\
\text { Exponencial }\end{array}$ & $\begin{array}{c}(2.74) \\
{[\text { Shalvi \& Weinstein, }} \\
\text { 1990, 1993] }\end{array}$ & $\begin{array}{c}\text { Proposição } 3 \\
\qquad(2.101) \\
\text { [Shalvi \& Weinstein, } \\
1990,1993]\end{array}$ & - \\
\hline $\begin{array}{l}\text { Gradiente } \\
\text { Projetado } \\
\text { (GP) }\end{array}$ & $\begin{array}{c}(2.77) \\
{[\text { Mboup \& }} \\
\text { Regalia, 2000] }\end{array}$ & - & $\begin{array}{c}(2.100) \\
{[\text { Mboup \& }} \\
\text { Regalia, 2000] }\end{array}$ \\
\hline $\begin{array}{c}\text { GP não- } \\
\text { normalizado }\end{array}$ & $(2.83)$ & $\begin{array}{c}\text { Proposição } 4 \\
(2.109)\end{array}$ & $\begin{array}{c}\text { Teorema } 1 \\
(2.86)\end{array}$ \\
\hline
\end{tabular}

Tabela 2.2: Resumo dos algoritmos e análises de convergência do Capítulo 2. 
Verificou-se que os algoritmos SD-CMA e SD-SWA minimizam efetivamente a função custo de Godard, dado que se utilizam as estatísticas do sinal transmitido ao invés das estatísticas do sinal de saída do equalizador. Desta forma, o SD-SWA pode ser interpretado como sendo o SD-CMA com um passo ótimo para velocidade de convergência, que utiliza a matriz de autocorrelação inversa.

Os algoritmos do tipo Super-Exponencial foram revisitados e um algoritmo do Gradiente Projetado não-normalizado proposto. Uma análise de convergência desse algoritmo foi apresentada no Teorema 1. Inspirando-se nesse teorema e reescrevendo o SD-CMA no domínio da resposta combinada, obteve-se o Teorema 2 que estabelece o intervalo do passo de adaptação para assegurar a convergência do algoritmo. Através de simulações, verificou-se que, se o passo de adaptação do SD-CMA for escolhido a cada instante de tempo de acordo com o Teorema 2, sua convergência será assegurada, independente da qualidade das estimativas e da margem de segurança do limite máximo do intervalo. Embora esse resultado seja uma contribuição importante na análise de algoritmos autodidatas, ele é válido apenas para o algoritmo que utiliza o gradiente determinístico, estimado de forma exata ou em bloco. Algoritmos desse tipo possuem uma elevada complexidade computacional, dado que estimativas em bloco devem ser calculadas a cada iteração. Na prática, é usual fazer simplificações ou considerações heurísticas com o intuito de diminuir a complexidade dos algoritmos [Benveniste et al., 1987]. Segundo o método da Seção 1.5, essa é a quarta e última etapa do projeto de algoritmos adaptativos. Os algoritmos simplificados devem então ser analisados segundo um método estatístico e testados em simulações, o que é feito no Capítulo 3.

A extensão do Teorema 2 para o algoritmo do Módulo Constante que utiliza o gradiente estocástico é um problema em aberto e de difícil solução. No entanto, o resultado obtido neste capítulo pode servir de inspiração, abrindo novas perspectivas para a solução de tal problema. 


\section{Capítulo 3}

\section{Algoritmos estocásticos para equalização monocanal}

Neste capítulo, são abordados algoritmos do gradiente estocástico para equalização monocanal. Inicialmente, os algoritmos do Módulo Constante e de Shalvi-Weinstein são revisitados. Em seguida, a partir da discretização de um algoritmo de controle adaptativo modificado, é proposto um novo algoritmo para equalização autodidata. Ele utiliza a segunda derivada ("aceleração") da estimativa dos coeficientes e é denominado algoritmo do Módulo Constante Acelerado. É feita uma análise de tracking dos algoritmos que satisfazem a relação de conservação de energia de [Sayed, 2003]. Além disso, abordam-se o problema da rotação de fase e os algoritmos concorrentes. O capítulo termina com resultados de simulações e conclusões.

\subsection{Algoritmos decorrentes da estimativa instantânea do gradiente}

Na Seção 2.4, abordaram-se os algoritmos decorrentes da função custo de Godard, baseados no método do gradiente determinístico. Neste caso, o vetor de coeficientes é atualizado na direção negativa do gradiente

$$
\nabla_{\mathbf{w}} J_{G}(n)=\mathrm{E}\left\{|y(n)|^{2} y(n) \mathbf{u}^{*}(n)\right\}-R_{2}^{a} \mathbf{R} \mathbf{w}(n-1) .
$$


Na prática, nem sempre é possível estimar de forma exata esse gradiente em cada iteração, dado que isso requer o conhecimento a priori da matriz de autocorrelação $\mathbf{R}$ e da esperança $\mathrm{E}\left\{|y(n)|^{2} y(n) \mathbf{u}^{*}(n)\right\}$. Uma alternativa é fazer estimativas em bloco. Neste caso, os algoritmos resultantes possuem uma complexidade computacional que aumenta com o tamanho do bloco de dados e geralmente não apresentam desempenho satisfatório em ambientes não-estacionários [Sayed, 2003]. Outra alternativa é estimar o gradiente a partir de dados disponíveis. Uma estratégia mais simples neste sentido é considerar estimativas instantâneas, o que resulta em algoritmos do gradiente estocástico [Haykin, 1996]. Assim, estimando (3.1) de forma instantânea, obtém-se

$$
\widehat{\nabla}_{\mathbf{w}} J_{G}(n)=e(n) \mathbf{u}^{*}(n)
$$

sendo

$$
e(n)=\left(|y(n)|^{2}-R_{2}^{a}\right) y(n)
$$

o sinal de "erro" de (2.59), reproduzido aqui por conveniência. Desta estimativa, chega-se à seguinte equação geral para algoritmos do gradiente estocástico

$$
\mathbf{w}(n)=\mathbf{w}(n-1)-\alpha(n) e(n) \mathbf{u}^{*}(n)
$$

sendo $\alpha(n)$ um passo de adaptação que, dependendo do algoritmo, pode ser um escalar positivo ou uma matriz.

No caso do algoritmo do Módulo Constante (CMA - Constant Modulus Algorithm) [Godard, 1980; Treichler \& Agee, 1983], o passo de adaptação é um escalar, ou seja, $\alpha(n)=\mu$. No caso do algoritmo de Shalvi-Weinstein (SWA - Shalvi-Weinstein Algorithm) [Shalvi \& Weinstein, 1990, 1993], utiliza-se uma estimativa do passo ótimo para velocidade de convergência de (2.65), ou seja,

$$
\alpha(n)=\frac{\widehat{\mathbf{R}}^{-1}(n)}{\gamma},
$$

sendo

$$
\widehat{\mathbf{R}}(n)=\sum_{l=0}^{n} \lambda^{n-l} \mathbf{u}^{*}(l) \mathbf{u}^{T}(l),
$$


$0 \ll \lambda<1$ um fator de esquecimento e $\gamma=\gamma^{a}=-C_{2,2}^{a} / C_{1,1}^{a}$. A inversa da matriz $\widehat{\mathbf{R}}(n)$, denotada por $\mathbf{P}=\widehat{\mathbf{R}}^{-1}$, é obtida aplicando-se o lema da inversão matricial em (3.6) [Haykin, 1996, p. 565]. As operações do CMA e SWA estão mostradas respectivamente nas tabelas 3.1 e 3.2. Considerando sinais reais, o primeiro requer $2 M+3$ multiplicações e $2 M$ somas por iteração, enquanto o segundo necessita de $4 M^{2}+3 M+3$ multiplicações, $3 M^{2}+M$ somas e 1 divisão.

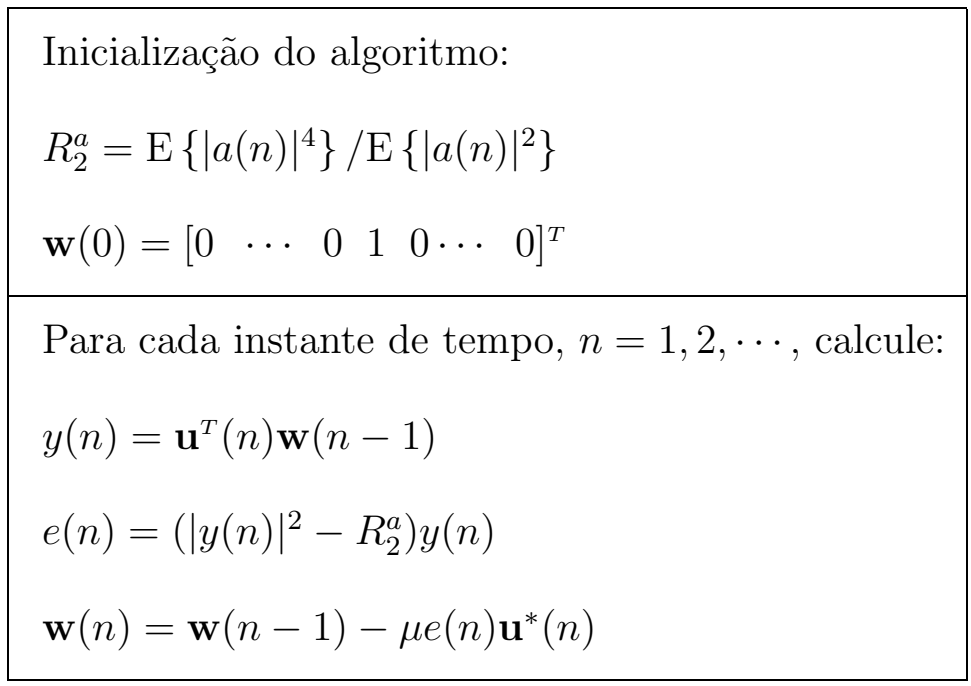

Tabela 3.1: Sumário do algoritmo do Módulo Constante (CMA).

Inicialização do algoritmo:

$$
\begin{aligned}
& R_{2}^{a}=\mathrm{E}\left\{|a(n)|^{4}\right\} / \mathrm{E}\left\{|a(n)|^{2}\right\} \\
& \mathbf{w}(0)=\left[\begin{array}{lllllll}
0 & \cdots & 0 & 1 & 0 & \cdots & 0
\end{array}\right]^{T} \\
& \mathbf{P}(0)=\delta \mathbf{I}, \quad \delta=\text { constante positiva pequena } \\
& \gamma=-C_{2,2}^{a} / C_{1,1}^{a}
\end{aligned}
$$

Para cada instante de tempo, $n=1,2, \cdots$, calcule:

$$
\begin{aligned}
& y(n)=\mathbf{u}^{T}(n) \mathbf{w}(n-1) \\
& e(n)=\left(|y(n)|^{2}-R_{2}^{a}\right) y(n) \\
& \mathbf{w}(n)=\mathbf{w}(n-1)-\gamma^{-1} \mathbf{P}(n-1) \mathbf{u}^{*}(n) e(n) \\
& \mathbf{P}(n)=\frac{1}{\lambda}\left[\mathbf{P}(n-1)-\frac{\mathbf{P}(n-1) \mathbf{u}^{*}(n) \mathbf{u}^{T}(n) \mathbf{P}(n-1)}{\lambda+\mathbf{u}^{T}(n) \mathbf{P}(n-1) \mathbf{u}^{*}(n)}\right]
\end{aligned}
$$

Tabela 3.2: Sumário do Algoritmo de Shalvi-Weinstein (SWA). 
Considerando os algoritmos do Módulo Constante e de Shalvi-Weinstein, podem-se estabelecer as relações apresentadas na Figura 3.1. A partir da função custo de Godard chega-se ao CMA através do método do gradiente estocástico, fazendo $\alpha(n)=\mu$. Partindo da função custo de Shalvi-Weinstein obtém-se o SWA, utilizando cumulantes empíricos e algumas aproximações estocásticas. Do CMA chega-se ao SWA, utilizando um passo ótimo para velocidade de convergência dado por $\alpha(n)=\widehat{\mathbf{R}}^{-1}(n) / \gamma$. Em contrapartida, a partir do SWA obtém-se o CMA, estimando a matriz de autocorrelação inversa como $\widehat{\mathbf{R}}^{-1}(n) \approx \gamma \mu \mathbf{I}$.

\section{Equivalência entre as funções custo}

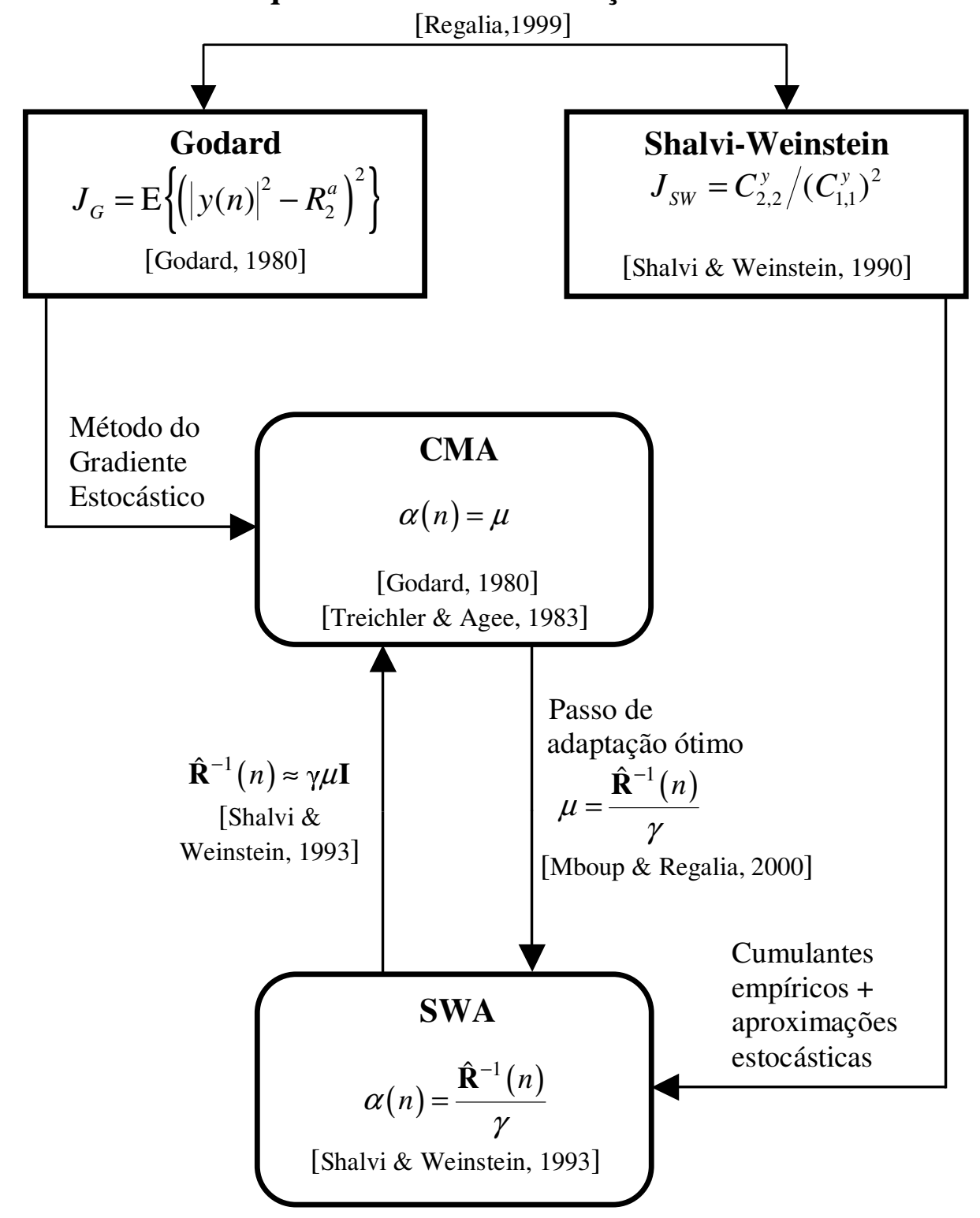

Figura 3.1: Relações entre algoritmos autodidatas clássicos. 
Cabe observar que das funções custo de Godard e de Shalvi-Weinstein, outros algoritmos podem ser obtidos, como os baseados no método quase-Newton. Neste caso, destacam-se o SNLA (Stochastic Newton-Like Algorithm) de [Yan \& Fan, 2000] e os algoritmos propostos em [Schirtzinger \& Jenkins, 1995].

\subsection{O algoritmo do Módulo Constante Acelerado}

Um algoritmo adaptativo de tempo discreto para filtragem adaptativa linear e supervisionada foi apresentado em [Jojoa, 1999; Gerken et al., 2000] e posteriormente analisado em [Jojoa et al., 2001b; Jojoa, 2003]. Ele foi obtido da discretização de um algoritmo de tempo contínuo para controle adaptativo, proposto em [Pait, 1998], que ajusta a segunda derivada ("aceleração") da estimativa dos coeficientes. Por essa razão, o algoritmo de tempo discreto foi denominado AAFA (Accelerating Adaptive Filtering Algorithm). Às custas de um moderado aumento da complexidade computacional, esse algoritmo pode apresentar um compromisso mais favorável entre velocidade de convergência e erro de estimação em regime que o dos algoritmos LMS ou NLMS (Normalized LMS) [Jojoa et al., 2001b; Jojoa, 2003].

Inspirando-se nesse algoritmo, em [Silva \& Gerken, 2000a; Silva, 2001], foi proposto um algoritmo do tipo "acelerador" para treinamento de redes neurais MLP (Multilayer Perceptrons) que apresentou uma velocidade de convergência maior que a do algoritmo Backpropagation e uma complexidade computacional inferior à de um algoritmo baseado no método dos mínimos quadrados recursivo.

Esses resultados motivaram o desenvolvimento de um algoritmo para equalização autodidata baseado nos mesmos princípios. Como o AAFA e o algoritmo do tipo "acelerador" para treinamento de redes MLP apresentam vantagens em relação ao algoritmos LMS e Backpropagation respectivamente, pode-se esperar que um algoritmo baseado na "aceleração" da estimativa dos coeficientes para equalização autodidata tenha um desempenho melhor que o do CMA. Como a origem desse algoritmo é diferente dos demais considerados neste trabalho, revisita-se inicialmente o algoritmo de tempo contínuo de [Pait, 1998]. Inspirando-se nas metodologias para projeto de algoritmos adaptativos da Seção 1.5, obtém-se em seguida o algoritmo do tipo "acelerador" para equalização autodidata, chamado de algoritmo do Módulo Constante Acelerado (AC-CMA - Accelerated Constant Modulus Algorithm). 


\subsubsection{O algoritmo "acelerador" de tempo contínuo}

Muitas vezes em controle adaptativo, é necessário ajustar recursivamente a estimativa $\mathbf{w}(t)$ do vetor de coeficientes $\mathbf{w}_{\mathrm{o}}$, usando um sinal desejado

$$
d(t)=\mathbf{u}^{T}(t) \mathbf{w}_{\mathrm{o}}+v_{m}(t)
$$

sendo $\mathbf{u}(t)$ o sinal de entrada (vetor regressor) e $v_{m}(t)$ o ruído de medida. O objetivo é manter o erro de estimação

$$
\varepsilon(t)=\mathbf{u}^{T}(t) \mathbf{w}(t)-d(t)
$$

e o erro dos coeficientes

$$
\widetilde{\mathbf{w}}(t)=\mathbf{w}_{\mathrm{o}}-\mathbf{w}(t)
$$

tão pequenos quanto possível. Neste contexto, é usual considerar um algoritmo que ajusta a primeira derivada ("velocidade") da estimativa dos coeficientes da forma

$$
\dot{\mathbf{w}}(t)=-\mathbf{M u}^{*}(t) \varepsilon(t),
$$

sendo M uma matriz positiva definida de dimensões $M \times M$.

O algoritmo que ajusta a segunda derivada ("aceleração") da estimativa dos coeficientes foi proposto em [Pait, 1998]. Observando que $\dot{\widetilde{\mathbf{w}}}(t)=-\dot{\mathbf{w}}(t)$ e definindo $\mathbf{g}(t)=\dot{\mathbf{w}}(t)$, o algoritmo "acelerador" pode ser descrito como

$$
\begin{aligned}
\dot{\mathbf{w}}(t) & =\mathbf{g}(t) \\
\dot{\mathbf{g}}(t) & =-\mathbf{u}^{*}(t) \varepsilon(t)-2\left(\mathbf{I}+\mathbf{u}^{*}(t) \mathbf{u}^{T}(t)\right) \mathbf{g}(t),
\end{aligned}
$$

sendo I a matriz identidade. Uma generalização de (3.11b) pode ser expressa como

$$
\dot{\mathbf{g}}(t)=-\mathbf{M}_{1} \mathbf{u}^{*}(t) \varepsilon(t)-2 \mathbf{M}_{1}\left(\mathbf{M}_{2}+\mathbf{u}^{*}(t) \mathbf{u}^{T}(t) \mathbf{M}_{1} \mathbf{M}_{3}\right) \mathbf{g}(t),
$$

sendo $\mathbf{M}_{k}, k=1,2,3$, matrizes simétricas, positiva-definidas e de dimensões $M \times M$. Essa generalização foi inspirada na matriz

$$
\mathbf{L}=\left[\begin{array}{cc}
\mathbf{M}_{2} & \mathbf{M}_{1}^{-1} / 2 \\
\mathbf{M}_{1}^{-1} / 2 & \mathbf{M}_{3}
\end{array}\right]
$$


usada na definição de uma função de Lyapunov. Utilizando as equações (3.7) e (3.8), a dinâmica desse algoritmo pode ser descrita em função do vetor de erro dos coeficientes $\widetilde{\mathbf{w}}(t)$, isto é,

$$
\left[\begin{array}{r}
-\dot{\widetilde{\mathbf{w}}}(t) \\
\dot{\mathbf{g}}(t)
\end{array}\right]=\left[\begin{array}{cc}
\mathbf{0} & \mathbf{I} \\
\mathbf{A}_{21} & \mathbf{A}_{22}
\end{array}\right]\left[\begin{array}{r}
-\widetilde{\mathbf{w}}(t) \\
\mathbf{g}(t)
\end{array}\right]+\left[\begin{array}{c}
\mathbf{0} \\
\mathbf{M}_{1} \mathbf{u}^{*}(t)
\end{array}\right] v_{m}(n),
$$

sendo

$$
\mathbf{A}_{21}=-\mathbf{M}_{1} \mathbf{u}^{*}(t) \mathbf{u}^{T}(t)
$$

e

$$
\mathbf{A}_{22}=-2 \mathbf{M}_{1}\left(\mathbf{M}_{2}+\mathbf{u}^{*}(t) \mathbf{u}^{T}(t) \mathbf{M}_{1} \mathbf{M}_{3}\right)
$$

As condições suficientes para assegurar a estabilidade desse sistema na ausência de ruído de medida, estabelecidas por uma função de Lyapunov baseada em (3.13) e obtidas em [Pait, 1998], são ${ }^{1}$

$$
4 \mathbf{M}_{1} \mathbf{M}_{3} \mathbf{M}_{1} \mathbf{M}_{2}>\mathbf{I}
$$

e

$$
\mathbf{M}_{2} \mathbf{M}_{1} \mathbf{M}_{3}+\mathbf{M}_{1} \mathbf{M}_{3} \mathbf{M}_{2}>\mathbf{M}_{1}^{-1} / 2
$$

\subsubsection{Métodos de discretização de Euler}

Dentre os diferentes métodos de discretização, os métodos de Euler progressivo e regressivo são os mais simples. Seja

$$
\dot{f}_{1}(t)=f_{2}(t)
$$

uma equação diferencial de primeira ordem [Simmons, 1987, p. 565] e $T$ um passo de integração. Discretizando-se (3.17) com o método de Euler progressivo [Press et al., 1986], obtém-se

$$
f_{1}(n+1)=f_{1}(n)+T f_{2}(n)
$$

e com o método de Euler regressivo [Press et al., 1986],

$$
f_{1}(n)=f_{1}(n-1)+T f_{2}(n) .
$$

\footnotetext{
${ }^{1}$ A notação $\mathbf{A}>\mathbf{B}$ significa que a matriz $(\mathbf{A}-\mathbf{B})$ é positiva-definida.
} 
Utilizando o método de Euler progressivo com $\mathbf{M}=\mu_{\mathrm{o}} \mathbf{I}$ e $\mu=\mu_{\mathrm{o}} T$, o algoritmo LMS pode ser obtido a partir da discretização das equações (3.10) e (3.8). No caso do AAFA, utilizou-se o método de Euler regressivo para discretizar (3.11a), (3.12) e (3.8). As razões para essa escolha são bem simples [Gerken et al., 2000; Jojoa, 2003]:

- o método de Euler progressivo resulta num algoritmo de baixa complexidade que nem sempre apresenta vantagens face ao LMS e que pode ser instável;

- outros métodos de integração, como o método do trapézio, resultam em algoritmos com elevada complexidade computacional.

A seguir, (3.8) é substituída por uma equação não-linear e o algoritmo do Módulo Constante Acelerado é obtido.

\subsubsection{O algoritmo "acelerador" para equalização autodidata}

Seja a equação de erro, decorrente da função custo de Godard [Godard, 1980],

$$
\varepsilon(t)=\varphi\left(y_{c}(t)\right)=\left(\left|y_{c}(t)\right|^{2}-R_{2}^{a}\right) y_{c}(t)
$$

em que $y_{c}(t)=\mathbf{u}^{T}(t) \mathbf{w}(t)$. Utilizando o método de Euler progressivo com $\mathbf{M}=\mu_{\mathrm{o}} \mathbf{I}$ e $\mu=\mu_{\mathrm{o}} T$, as equações do CMA podem ser obtidas a partir da discretização de (3.10) e (3.18). No caso do algoritmo do Módulo Constante Acelerado (AC-CMA), pelas mesmas razões do AAFA, utiliza-se o método de Euler regressivo. Sua dedução é resumida pelos seguintes passos:

1. aplicação do método de Euler regressivo para discretizar as equações (3.11a), (3.12) e (3.18) com passo de integração $\mu$;

2. introdução de um erro a priori definido como

$$
e(n)=\varphi\left(\mathbf{u}^{T}(n) \mathbf{w}(n-1)\right)
$$

3. utilização de uma aproximação linear de $\varphi(\cdot)$ (expansão em série de Taylor de $1^{\underline{a}}$ ordem em torno de $\mathbf{w}_{\mathbf{v}}$ ) descrita por

$$
\begin{aligned}
\varphi\left(\mathbf{u}^{T} \mathbf{w}\right) & \approx \varphi\left(\mathbf{u}^{T} \mathbf{w}_{\mathbf{v}}\right)+\left[\left.\frac{\partial \varphi\left(\mathbf{u}^{T} \mathbf{w}\right)}{\partial \mathbf{w}}\right|_{\mathbf{w}=\mathbf{w}_{\mathrm{v}}}\right]^{T}\left(\mathbf{w}-\mathbf{w}_{\mathbf{v}}\right) \\
& =\varphi\left(\mathbf{u}^{T} \mathbf{w}_{\mathbf{v}}\right)+\breve{\varphi}\left(\mathbf{u}^{T} \mathbf{w}_{\mathbf{v}}\right) \mathbf{u}^{T}\left(\mathbf{w}-\mathbf{w}_{\mathbf{v}}\right)
\end{aligned}
$$

sendo $\breve{\varphi}(y)=\beta|y|^{2}-R_{2}^{a}$ e $\beta=2(=3)$ para o caso complexo (real); 
4. consideração de $\mathbf{M}_{k}=m_{k} \mathbf{I}, k=1,2,3$, sendo $m_{k}$ constantes positivas e $\mathbf{I}$ a matriz identidade;

5. imposição das relações $m_{1} m_{2}=1 /(2 \kappa)$ e $m_{1} m_{3}=\kappa / 2$. Essas relações foram motivadas pelo fato de que o AAFA é estável e atinge sua maior velocidade de convergência, se a condição $4 m_{1}^{2} m_{2} m_{3}=1$ é satisfeita [Gerken et al., 2000]. Esses resultados foram demonstrados apenas para o caso supervisionado, mas também são usados no autodidata. Neste caso, assume-se que a estimativa do sinal desejado seja próxima de $\mathbf{u}^{T}(n) \mathbf{w}_{\mathrm{o}}$. Embora essa consideração não seja muito realista, resultados de simulações sugerem que com $4 m_{1}^{2} m_{2} m_{3}=1$ e $0<\mu<1$, o AC-CMA é estável, apresentando um desempenho que depende do ajuste dos parâmetros $\kappa, m_{1}$ e $\mu$.

A obtenção do AC-CMA e do CMA a partir dos algoritmos de tempo contínuo é esquematizada na Figura 3.2.

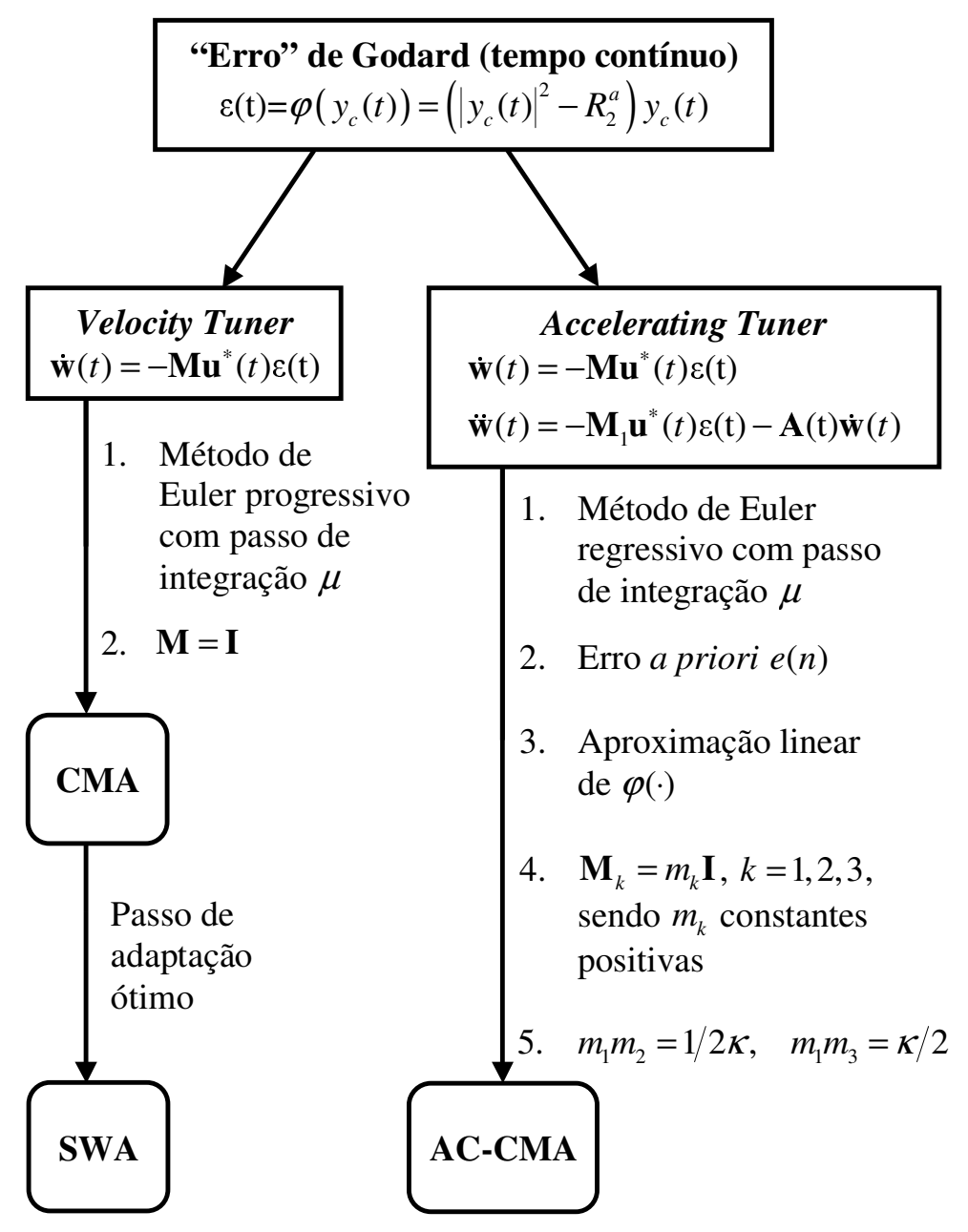

Figura 3.2: Relações entre os algoritmos de tempo contínuo e algoritmos autodidatas, $\mathbf{A}(t)=2 \mathbf{M}_{1}\left(\mathbf{M}_{2}+\mathbf{u}^{*}(t) \mathbf{u}^{T}(t) \mathbf{M}_{1} \mathbf{M}_{3}\right)$. 
As operações do AC-CMA são apresentadas na Tabela 3.3. Uma dedução detalhada do algoritmo é feita no Apêndice C. Cabe observar que $\mu m_{1} \kappa^{2}, \mu^{2} m_{1} \kappa, \mu m_{1}(\mu+\kappa), \mu+\kappa$ e $\kappa /(\mu+\kappa)$ precisam ser calculados apenas uma única vez. Para sinais reais, esta versão requer $6 M+6$ multiplicações, 1 divisão e $5 M+4$ adições, uma complexidade computacional que fica entre a do CMA e a do SWA.

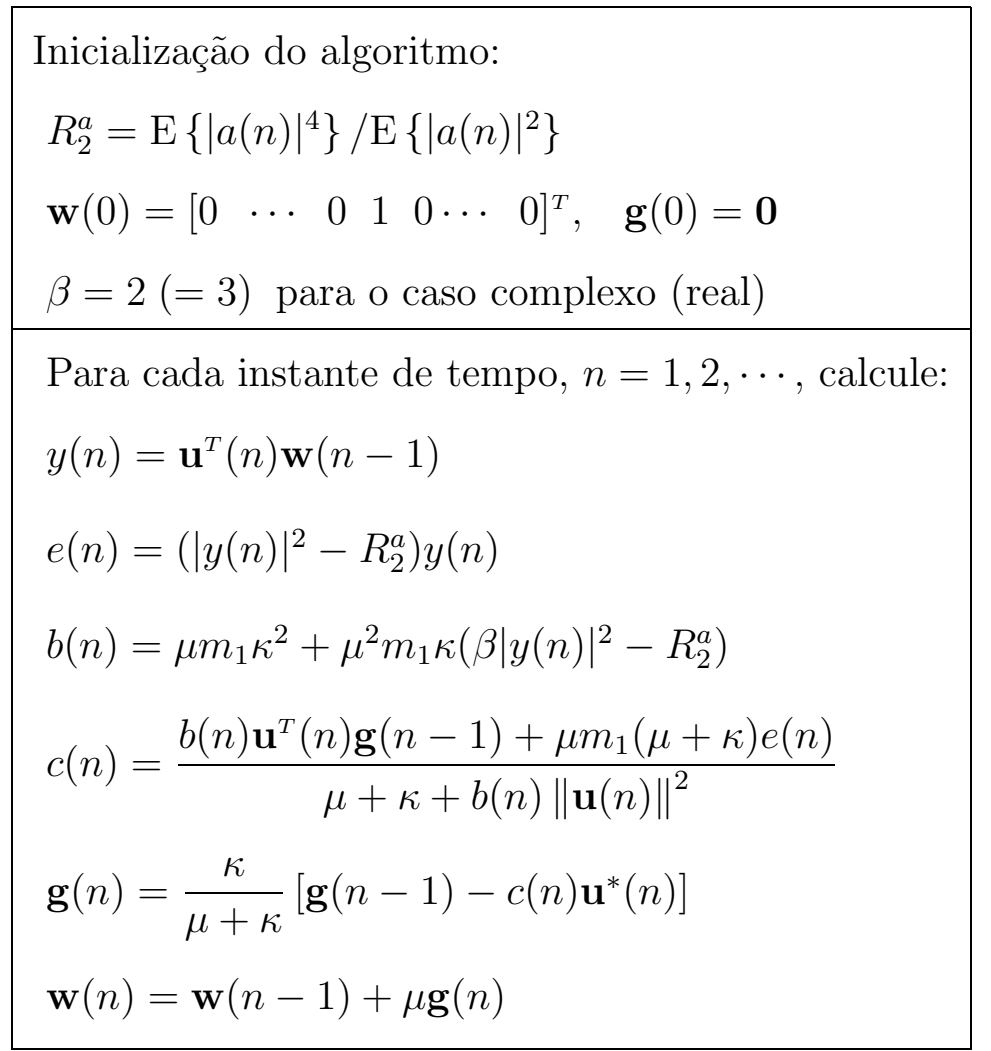

Tabela 3.3: Sumário do Algoritmo do Módulo Constante Acelerado (AC-CMA).

\subsection{Os algoritmos segundo o método quase-Newton}

O método de Newton minimiza de forma exata uma aproximação quadrática local da função custo $J(\mathbf{w})$ [Luenberger, 1984]. Desta forma, próximo de $\mathbf{w}(n-1)$, pode-se aproximar $J(\mathbf{w})$ por uma série de Taylor truncada

$$
\begin{aligned}
J(\mathbf{w}) \simeq J(\mathbf{w}(n-1))+\frac{1}{2}\left\{\nabla_{\mathbf{w}}^{H} J(n)[\mathbf{w}-\mathbf{w}(n-1)]+[\mathbf{w}-\mathbf{w}(n-1)]^{H} \nabla_{\mathbf{w}} J(n)\right\} \\
+\frac{1}{2}[\mathbf{w}-\mathbf{w}(n-1)]^{H} \mathbf{F}(n)[\mathbf{w}-\mathbf{w}(n-1)],
\end{aligned}
$$

sendo $\mathbf{F}(n)$ a hessiana da função $J(\mathbf{w})$. O lado direito dessa expressão é minimizado através do seguinte algoritmo

$$
\mathbf{w}(n)=\mathbf{w}(n-1)-\mathbf{F}^{-1}(n) \boldsymbol{\nabla}_{\mathbf{w}} J(n),
$$


que caracteriza o algoritmo de Newton [Luenberger, 1984].

Embora a convergência desse método seja atrativa, o cálculo da matriz hessiana inversa não é adequado para uma implementação prática. Em contrapartida, o método quaseNewton usa aproximações da inversa dessa matriz e também do vetor gradiente [Luenberger, 1984]. Uma primeira modificação nessa direção é a introdução de um passo de adaptação $\rho(n)$, ou seja,

$$
\mathbf{w}(n)=\mathbf{w}(n-1)-\rho(n) \widehat{\mathbf{F}}^{-1}(n) \widehat{\nabla}_{\mathbf{w}} J(n)
$$

Os algoritmos do Módulo Constante, de Shalvi-Weinstein e do Módulo Constante Acelerado podem ser interpretados e comparados utilizando o método quase-Newton. Quanto ao CMA e ao SWA, as aproximações para os vetores gradientes e matrizes hessianas são obtidas diretamente da observação das equações mostradas respectivamente nas tabelas 3.1 e 3.2. A interpretação do AC-CMA segundo o método quase-Newton é apresentada na Seção C.2 do Apêndice C.

Na Tabela 3.4, é apresentado um resumo das interpretações desses algoritmos. O algoritmo do tipo quase-Newton proposto em [Yan \& Fan, 2000], chamado de SNLA (Stochastic Newton-like Algorithm), também é considerado para comparação. A matriz $\widehat{\mathbf{F}}(n)$ utilizada por ele é uma aproximação instantânea da hessiana da função custo de Godard e $\varsigma>0$ é o fator de convergência. Cabe observar que, quanto ao gradiente, o AC-CMA utiliza uma aproximação um pouco mais complexa que as aproximações do SWA, do SNLA e do CMA. Em contrapartida, quanto à hessiana, o SWA e o SNLA utilizam aproximações que exigem mais operações, o que faz com que esses algoritmos tenham uma complexidade computacional da ordem de $M^{2}$. A aproximação da matriz hessiana para o AC-CMA é apenas uma interpretação dada segundo o método quase-Newton. Na realidade, não é necessário fazer o cálculo da inversa dessa matriz como pode ser visto na Tabela 3.3. Desta forma, o AC-CMA apresenta uma complexidade computacional que fica entre a do CMA e a do SWA ou SNLA. É importante ressaltar que esses algoritmos também podem ser interpretados como algoritmos do gradiente estocástico. Neste caso, o passo de adaptação deixa de ser um escalar e passa a ser uma matriz dada por $\alpha(n)=\rho(n) \widehat{\mathbf{F}}^{-1}(n)$. 


\begin{tabular}{|c|c|c|c|}
\hline Alg. & $\rho(n)$ & $\widehat{\nabla}_{\mathbf{w}} J(n)$ & $\widehat{\mathbf{F}}(n)$ \\
\hline CMA & $\mu$ & $e(n) \mathbf{u}^{*}(n)$ & I \\
\hline SWA & $\gamma^{-1}$ & $e(n) \mathbf{u}^{*}(n)$ & $\sum_{l=1}^{n} \lambda^{n-l} \mathbf{u}^{*}(l) \mathbf{u}^{T}(l)$ \\
\hline SNLA & $\varsigma \frac{1-\lambda^{n}}{1-\lambda}$ & $e(n) \mathbf{u}^{*}(n)$ & $\sum_{l=1}^{n} \lambda^{n-l}\left(\beta|y(l)|^{2}-R_{2}^{a}\right) \mathbf{u}^{*}(l) \mathbf{u}(l)$ \\
\hline AC-CMA & $\frac{\mu^{2} m_{1} \kappa}{\mu+\kappa}$ & $\begin{array}{c}e(n) \mathbf{u}^{*}(n) \\
-\frac{1}{\mu^{2} m_{1}}\{\mathbf{w}(n-1)-\mathbf{w}(n-2)\}\end{array}$ & $\begin{array}{c}\rho(n)\left(\beta|y(n)|^{2}-R_{2}^{a}\right) \mathbf{u}^{*}(n) \mathbf{u}^{T}(n) \\
+\frac{\kappa}{\mu} \rho(n) \mathbf{u}^{*}(n) \mathbf{u}^{T}(n)+\mathbf{I}\end{array}$ \\
\hline
\end{tabular}

Tabela 3.4: Interpretação dos algoritmos aproximados segundo o método quase-Newton.

\subsection{A relação de conservação de energia}

A relação de conservação de energia foi explorada no contexto de análise de robustez e estabilidade de filtros adaptativos por Rupp e Sayed (1996). Além disso, ela tem sido utilizada no estudo de convergência e tracking de esquemas adaptativos supervisionados e autodidatas [Yousef \& Sayed, 1999, 2001; Mai \& Sayed, 2000; Sayed, 2003; Silva \& Miranda, 2004a]. Em [Sayed, 2003, Cap. 6], é mostrado que algoritmos da forma

$$
\mathbf{w}(n)=\mathbf{w}(n-1)-\rho(n) \widehat{\mathbf{F}}^{-1}(n) \mathbf{u}^{*}(n) e(n)
$$

obedecem a relação de conservação de energia e podem ser descritos por um mapeamento sem perdas com realimentação.

Assumindo que $\mathbf{w}_{\mathrm{o}}$ é o vetor de coeficientes ótimos num ambiente estacionário, define-se o vetor de erro dos coeficientes como

$$
\widetilde{\mathbf{w}}(n)=\mathbf{w}_{\mathrm{o}}-\mathbf{w}(n) .
$$

Subtraindo ambos os lados de (3.24) de $\mathbf{w}_{\mathrm{o}}$, obtém-se

$$
\widetilde{\mathbf{w}}(n)=\widetilde{\mathbf{w}}(n-1)+\rho(n) \widehat{\mathbf{F}}^{-1}(n) \mathbf{u}^{*}(n) e(n) .
$$


Definindo os erros a priori e a posteriori como

$$
e_{a}(n) \triangleq \mathbf{u}^{T}(n) \widetilde{\mathbf{w}}(n-1)
$$

e

$$
e_{p}(n) \triangleq \mathbf{u}^{T}(n) \widetilde{\mathbf{w}}(n)
$$

e multiplicando ambos os lados de (3.26) por $\mathbf{u}^{T}(n)$ chega-se à seguinte relação

$$
e_{p}(n)=e_{a}(n)+\rho(n)\|\mathbf{u}(n)\|_{\widehat{\mathbf{F}}^{-1}}^{2} e(n),
$$

sendo $\|\mathbf{u}(n)\|_{\widehat{\mathbf{F}}^{-1}}^{2} \triangleq \mathbf{u}^{T}(n) \widehat{\mathbf{F}}^{-1}(n) \mathbf{u}^{*}(n)$. Essa relação pode ser reescrita como

$$
e(n)=-\frac{\bar{\rho}(n)}{\rho(n)}\left[e_{a}(n)-e_{p}(n)\right]
$$

sendo

$$
\bar{\rho}(n) \triangleq \begin{cases}\frac{1}{\|\mathbf{u}(n)\|_{\widehat{\mathbf{F}}^{-1}}^{2}}, & \mathbf{u}(n) \neq \mathbf{0} \\ 0, & \text { caso contrário. }\end{cases}
$$

Substituindo (3.30) em (3.26) obtém-se

$$
\widetilde{\mathbf{w}}(n)+\bar{\rho}(n) \widehat{\mathbf{F}}^{-1}(n) \mathbf{u}^{*}(n) e_{a}(n)=\widetilde{\mathbf{w}}(n-1)+\bar{\rho}(n) \widehat{\mathbf{F}}^{-1}(n) \mathbf{u}^{*}(n) e_{p}(n)
$$

Calculando a norma ao quadrado de ambos os lados de (3.32), utilizando $\widehat{\mathbf{F}}(n)$ como matriz de ponderação, chega-se a

$$
\|\widetilde{\mathbf{w}}(n)\|_{\widehat{\mathbf{F}}(n)}^{2}+\bar{\rho}(n)\left|e_{a}(n)\right|^{2}=\|\widetilde{\mathbf{w}}(n-1)\|_{\widehat{\mathbf{F}}(n)}^{2}+\bar{\rho}(n)\left|e_{p}(n)\right|^{2} .
$$

Esta é a relação de conservação de energia para algoritmos da forma (3.24). Ela estabelece que o mapeamento das variáveis $\left\{\widehat{\mathbf{F}}^{1 / 2}(n) \widetilde{\mathbf{w}}(n-1), \sqrt{\bar{\rho}(n)} e_{p}(n)\right\}$ para as variáveis $\left\{\widehat{\mathbf{F}}^{1 / 2}(n) \widetilde{\mathbf{w}}(n), \sqrt{\bar{\rho}(n)} e_{a}(n)\right\}$ conserva a energia. Combinando (3.29) e (3.33), obtém-se uma configuração com realimentação mostrada na Figura 3.3.

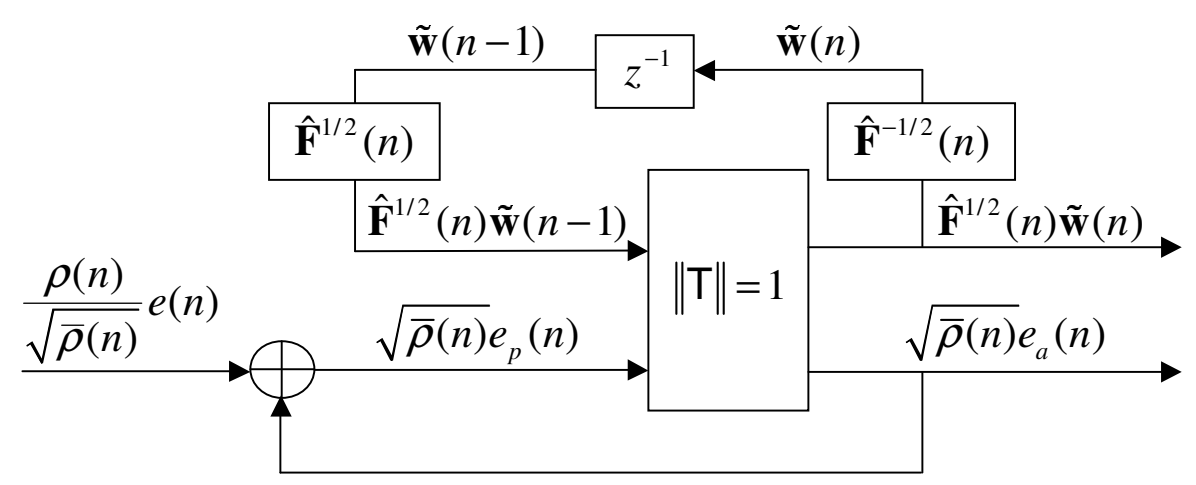

Figura 3.3: Mapeamento sem perdas com realimentação para algoritmos da forma (3.24). 
Seja o operador de projeção oblíqua no espaço ortogonal a $\mathbf{u}(n)$ na direção de $\widehat{\mathbf{F}}^{-1}(n) \mathbf{u}^{*}(n)$, dado por

$$
\mathcal{V}(n)=\mathbf{I}-\frac{\widehat{\mathbf{F}}^{-1}(n) \mathbf{u}^{*}(n) \mathbf{u}^{T}(n)}{\|\mathbf{u}(n)\|_{\widehat{\mathbf{F}}^{-1}}^{2}}
$$

Multiplicando (3.32) por $\mathcal{V}(n)$, obtém-se a seguinte relação

$$
\mathcal{V}(n) \widetilde{\mathbf{w}}(n)=\mathcal{V}(n) \widetilde{\mathbf{w}}(n-1)
$$

Todos os esquemas adaptativos que obedecem a relação de conservação de energia, também satisfazem (3.35).

Entre os algoritmos da Tabela 3.4, o CMA, o SWA e o SNLA possuem a forma (3.24) e portanto, satisfazem a relação (3.35). O AC-CMA utiliza uma estimativa do gradiente "corrigida" por um termo proporcional ao incremento do vetor de coeficientes. Conseqüentemente, ele não satisfaz uma relação do tipo (3.35) para os vetores $\widetilde{\mathbf{w}}(n)$ ou $\mathbf{g}(n)$. Em contrapartida, definindo uma combinação linear destes vetores como

$$
\widetilde{\mathbf{g}}(n) \triangleq \kappa \mathbf{g}(n)-\widetilde{\mathbf{w}}(n)
$$

e os erros a priori e a posteriori como

$$
\bar{\xi}_{a}(n) \triangleq \mathbf{u}^{T}(n) \widetilde{\mathbf{g}}(n-1)
$$

e

$$
\bar{\xi}_{p}(n) \triangleq \mathbf{u}^{T}(n) \widetilde{\mathbf{g}}(n)
$$

é demonstrado na Seção C.3 do Apêndice C que o AC-CMA obedece a uma relação equivalente a (3.35) para o vetor $\widetilde{\mathbf{g}}(n)$. Desta forma, ele também pode ser descrito por um mapeamento sem perdas com realimentação de ganho, como mostrado na Figura 3.4, sendo $\bar{\mu}(n)=\bar{\rho}(n) \operatorname{com} \widehat{\mathbf{F}}(n)=\mathbf{I}$. Embora esse resultado seja interessante, sua utilização na análise de convergência do algoritmo é um problema em aberto, dado que o AC-CMA também depende do erro a priori $e_{a}(n)$, definido em (3.27). 


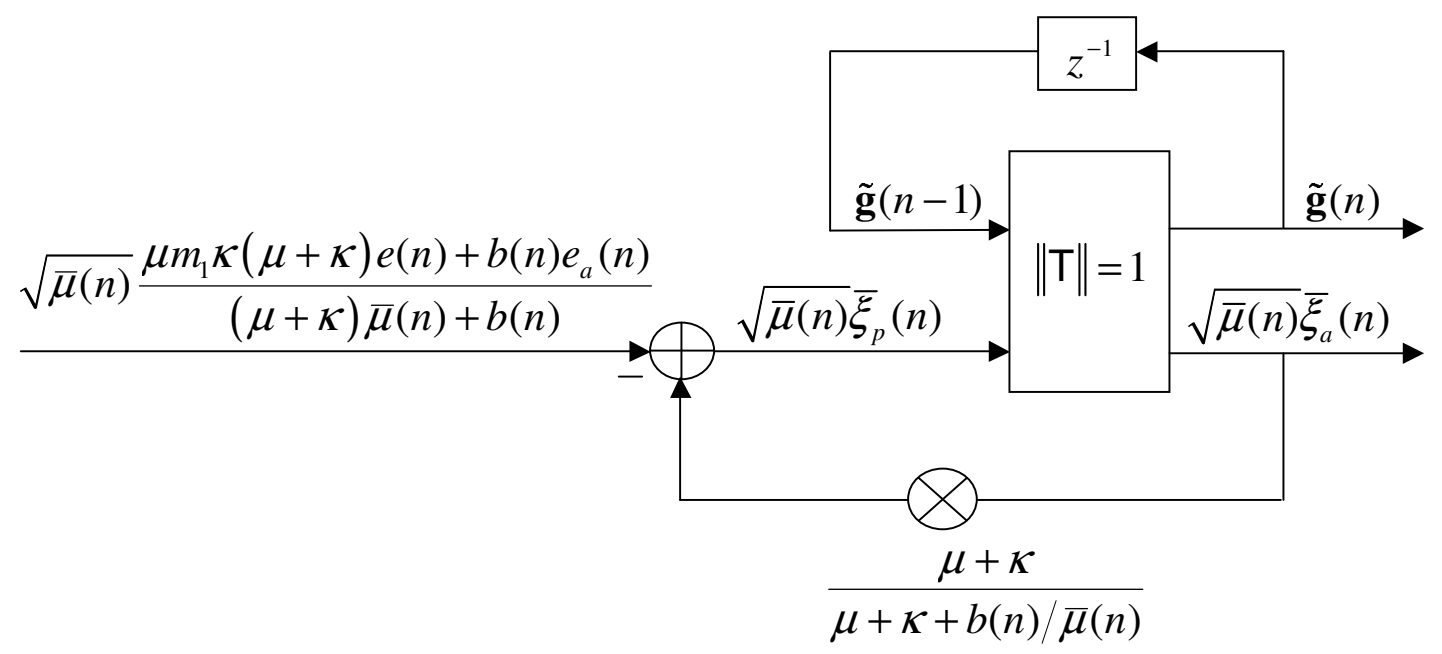

Figura 3.4: Mapeamento sem perdas com realimentação de ganho para o AC-CMA.

\subsection{Análise de tracking}

Num ambiente não-estacionário, assume-se que a variação dos coeficientes da solução ótima de zero-forcing $\mathbf{w}_{\mathrm{o}}$ segue o modelo [Eweda, 1994; Haykin, 1996; Sayed, 2003, Cap. 7]

$$
\mathbf{w}_{\mathrm{o}}(n)=\mathbf{w}_{\mathrm{o}}(n-1)+\mathbf{q}(n)
$$

sendo $\mathbf{q}(n)$ uma seqüência i.i.d., cuja matriz de autocorrelação $\mathbf{Q}=\mathrm{E}\left\{\mathbf{q}^{*}(n) \mathbf{q}^{T}(n)\right\}$ é positiva-definida. Além disso, assume-se que tal seqüência é independente das condições iniciais $\left\{\mathbf{w}_{\mathrm{o}}(-1), \mathbf{w}(-1)\right\}$ e de $\{\mathbf{u}(l)\}$ para todo $l<n$ [Sayed, 2003, Seção 7.4]. Neste caso, considera-se um pequeno grau de não-estacionariedade.

A Equação (3.26) pode ser reescrita no caso não-estacionário como

$$
\widetilde{\mathbf{w}}(n)-\mathbf{q}(n)=\widetilde{\mathbf{w}}(n-1)+\rho(n) \widehat{\mathbf{F}}^{-1}(n) \mathbf{u}^{*}(n) e(n) .
$$

Uma medida de desempenho usualmente empregada na análise de algoritmos adaptativos é o erro quadrático médio em regime (steady-state MSE), definido como

$$
\zeta \triangleq \lim _{n \rightarrow \infty} \mathrm{E}\left\{\left|e_{a}(n)\right|^{2}\right\}
$$

sendo $e_{a}(n)$ o erro a priori de (3.27). A análise de MSE e tracking de algoritmos da forma (3.24) é feita com as seguintes suposições: 
S1. $\mathrm{E}\{a(n)\}=0, \gamma>0$ e para sinais complexos $\mathrm{E}\left\{a^{2}(n)\right\}=0$ (simetria circular). Essa consideração é válida para a maior parte das constelações utilizadas em comunicações digitais [Shalvi \& Weinstein, 1994; Sayed, 2003].

S2. Algoritmos da forma (3.24) obedecem a seguinte condição de regime $(n \rightarrow \infty)$ [Sayed, 2003, Seção 6.9.3]

$$
\mathrm{E}\left\{\|\widetilde{\mathbf{w}}(n)\|_{\widehat{\mathbf{F}}}^{2}\right\}=\mathrm{E}\left\{\|\widetilde{\mathbf{w}}(n-1)\|_{\widehat{\mathbf{F}}}^{2}\right\}
$$

S3. $a\left(n-\tau_{d}\right)$ e $e_{a}(n)$ são independentes em regime. Essa consideração foi assumida na análise de MSE e tracking do CMA [Yousef \& Sayed, 1999] e de acordo com [Mai \& Sayed, 2000], ela é razoável já que requer essencialmente que em regime o erro de estimação $\left\{e_{a}(n)\right\}$ do equalizador seja insensível aos símbolos transmitidos $\{a(n)\}$ [Mai \& Sayed, 2000, As. I.1, p. 84].

S4. $\|\mathbf{u}(n)\|_{\widehat{\mathbf{F}}^{-1}}^{2}$ e $e(n)$ são independentes em regime. Isto requer que a energia ponderada do vetor de entrada seja independente da saída do equalizador. Uma consideração similar foi assumida em [Mai \& Sayed, 2000, As. I.2, p. 84].

S5. A Equação (3.3, página 72 ) pode ser reescrita utilizando-se a aproximação $y(n) \approx$ $a\left(n-\tau_{d}\right)-e_{a}(n)$, já que em regime $a\left(n-\tau_{d}\right) \approx \mathbf{u}^{T}(n) \mathbf{w}_{\mathrm{o}}(n-1)$.

S6. $\lim _{n \rightarrow \infty} \mathrm{E}\left\{e_{a}^{*}(n) e(n)+e_{a}(n) e^{*}(n)\right\} \approx-2 \gamma \zeta$. Essa consideração é obtida usando S3, S5 e assumindo que $\gamma \mathrm{E}\left\{\left|e_{a}(n)\right|^{2}\right\} \gg \mathrm{E}\left\{\left|e_{a}(n)\right|^{4}\right\}$ [Mai \& Sayed, 2000, Th. 3 e Th. 4].

S7. $\lim _{n \rightarrow \infty} \mathrm{E}\left\{|e(n)|^{2}\right\} \approx \mathrm{E}\left\{|a(n)|^{6}-\left(R_{2}^{a}\right)^{2}|a(n)|^{2}\right\} \triangleq \xi$. Para se obter essa consideração, além do uso de S1, S3 e S5, os termos que dependem de $\mathrm{E}\left\{\left|e_{a}(n)\right|^{l}\right\} \operatorname{com} l=2,4,6$, foram assumidos pequenos em relação a $\xi$ [Mai \& Sayed, 2000, Th. 3 e Th. 4]. Ela também pode ser obtida, assumindo-se a aproximação $\mathrm{E}\left\{|y(n)|^{l}\right\} \approx \mathrm{E}\left\{|a(n)|^{l}\right\} \operatorname{com} l=$ 2, 4,6, na média quadrática de (3.3).

As considerações S6 e S7 apresentam uma boa concordância com as simulações para constelações de módulo não-constante. Cabe observar que para constelações de módulo constante, $\xi=0$ e a análise a seguir só será válida ao se considerar sobre-amostragem [Mai \& Sayed, 2000]. Pode-se então estabelecer o seguinte Teorema [Silva \& Miranda, 2004a]. 
Teorema 3 Sob as considerações S1-S7, o erro médio quadrático em regime $(n \rightarrow \infty)$ dos algoritmos da forma (3.24) pode ser aproximado por

$$
\zeta \approx \frac{1}{2 \gamma \rho(n)}\left(\rho^{2}(n) \mathrm{E}\left\{\|\mathbf{u}(n)\|_{\widehat{\mathbf{F}}^{-1}}^{2}\right\} \xi+\mathrm{E}\left\{\|\mathbf{q}(n)\|_{\widehat{\mathbf{F}}}^{2}\right\}\right) .
$$

Demonstração: Calculando a norma ponderada ao quadrado de ambos os lados da Equação (3.40), usando $\widehat{\mathbf{F}}(n)$ como matriz de ponderação, obtém-se

$$
\begin{gathered}
\|\widetilde{\mathbf{w}}(n)-\mathbf{q}(n)\|_{\widehat{\mathbf{F}}}^{2}=\|\widetilde{\mathbf{w}}(n-1)\|_{\widehat{\mathbf{F}}}^{2}+\rho(n)\left[e(n) e_{a}^{*}(n)+e^{*}(n) e_{a}(n)\right] \\
+\rho^{2}(n)\|\mathbf{u}(n)\|_{\widehat{\mathbf{F}}^{-1}}^{2}|e(n)|^{2}
\end{gathered}
$$

Tomando a esperança de ambos os lados de (3.42) e usando $\mathrm{S} 2$, chega-se a

$$
-\mathrm{E}\left\{e_{a}^{*}(n) e(n)+e_{a}(n) e^{*}(n)\right\} \approx \rho(n) \mathrm{E}\left\{\|\mathbf{u}(n)\|_{\widehat{\mathbf{F}}^{-1}}^{2}|e(n)|^{2}\right\}+\rho^{-1}(n) \mathrm{E}\left\{\|\mathbf{q}(n)\|_{\widehat{\mathbf{F}}}^{2}\right\}
$$

Usando S4, S6 e S7 em (3.43), obtém-se (3.41), o que completa a demonstração.

Cabe notar que o MSE de (3.41) possui dois termos: um decorrente da estacionariedade e outro da não-estacionariedade. As expressões do MSE em regime para o CMA, SWA e SNLA são obtidas do Teorema 3, assumindo-se aproximações usuais para $\mathrm{E}\left\{\|\mathbf{u}(n)\|_{\widehat{\mathbf{F}}^{-1}}^{2}\right\}$ e $\mathrm{E}\left\{\|\mathbf{q}(n)\|_{\widehat{\mathbf{F}}}^{2}\right\}$ [Eweda, 1994; Sayed, 2003, páginas 320 e 382]. Num ambiente sem ruído e para constelações de módulo não-constante, é possível obter o parâmetro de adaptação ótimo, que minimiza o MSE em regime. Esse parâmetro é denotado genericamente como $\nu_{\mathrm{o}}$ e como $\mu_{o}, \lambda_{o}$ e $\varsigma_{o}$ para o CMA, o SWA e o SNLA respectivamente. Os correspondentes valores mínimos, denotados por $\zeta_{o}$, também podem ser calculados. Esses resultados são resumidos na Tabela 3.5. A análise de tracking do CMA [Yousef \& Sayed, 1999] é considerada aqui para comparação. Na análise do SNLA, quando $n \rightarrow \infty$, assume-se a seguinte aproximação

$$
\mathrm{E}\left\{\left(\beta|y(n)|^{2}-R_{2}^{a}\right) \mathbf{u}^{*}(n) \mathbf{u}^{T}(n)\right\} \approx \gamma \mathrm{E}\left\{\mathbf{u}^{*}(n) \mathbf{u}^{T}(n)\right\}=\gamma \mathbf{R}
$$

Da Tabela 3.5, conclui-se que, se $\varsigma_{\mathrm{o}}=1-\lambda_{\mathrm{o}}$, o SNLA e o SWA atingem o mesmo MSE em regime. Além disso, comparando o valor mínimo do MSE, obtêm-se as seguintes razões

$$
\frac{\zeta_{O}^{\mathrm{SNLA}}}{\zeta_{O}^{\mathrm{SWA}}}=1
$$

e

$$
\frac{\zeta_{o}^{\mathrm{SNLA}}}{\zeta_{O}^{\mathrm{CMA}}}=\frac{\zeta_{o}^{\mathrm{SWA}}}{\zeta_{O}^{\mathrm{CMA}}}=\sqrt{\frac{M \operatorname{Tr}(\mathbf{Q R})}{\operatorname{Tr}(\mathbf{R}) \operatorname{Tr}(\mathbf{Q})}}
$$




\begin{tabular}{|c|c|c|c|}
\hline Algoritmo & CMA & SNLA & SWA \\
\hline $\mathrm{E}\left\{\|\mathbf{u}(n)\|_{\widehat{\mathbf{F}}^{-1}}^{2}\right\}$ & $\operatorname{Tr}(\mathbf{R})$ & $\frac{(1-\lambda) M}{\gamma}$ & $(1-\lambda) M$ \\
\hline $\mathrm{E}\left\{\|\mathbf{q}(n)\|_{\widehat{\mathbf{F}}}^{2}\right\}$ & $\operatorname{Tr}(\mathbf{Q})$ & $\frac{\gamma \operatorname{Tr}(\mathbf{Q R})}{1-\lambda}$ & $\frac{\operatorname{Tr}(\mathbf{Q R})}{1-\lambda}$ \\
\hline$\zeta$ & $\frac{\mu \xi \operatorname{Tr}(\mathbf{R})}{2 \gamma}+\frac{\operatorname{Tr}(\mathbf{Q})}{2 \gamma \mu}$ & $\frac{M \varsigma \xi}{2 \gamma^{2}}+\frac{\operatorname{Tr}(\mathbf{Q R})}{2 \varsigma}$ & $\frac{M(1-\lambda) \xi}{2 \gamma^{2}}+\frac{\operatorname{Tr}(\mathbf{Q R})}{2(1-\lambda)}$ \\
\hline$\nu_{\mathrm{o}}$ & $\mu_{o}=\sqrt{\frac{\operatorname{Tr}(\mathbf{Q})}{\xi \operatorname{Tr}(\mathbf{R})}}$ & $\varsigma_{o}=\sqrt{\frac{\gamma^{2} \operatorname{Tr}(\mathbf{Q R})}{M \xi}}$ & $1-\lambda_{o}=\sqrt{\frac{\gamma^{2} \operatorname{Tr}(\mathbf{Q R})}{M \xi}}$ \\
\hline$\zeta_{o}$ & $\sqrt{\gamma^{-2} \xi \operatorname{Tr}(\mathbf{R}) \operatorname{Tr}(\mathbf{Q})}$ & $\sqrt{\gamma^{-2} M \xi \operatorname{Tr}(\mathbf{Q R})}$ & $\sqrt{\gamma^{-2} M \xi \operatorname{Tr}(\mathbf{Q R})}$ \\
\hline
\end{tabular}

Tabela 3.5: MSE $(\zeta)$ em regime, parâmetros de adaptação ótimos $\left(\nu_{\mathrm{o}}\right)$ e valores mínimos do MSE em regime $\left(\zeta_{o}\right)$ para o CMA, SNLA e SWA.

A razão (3.44) era esperada já que o SWA e o SNLA possuem o mesmo comportamento em regime. A razão (3.45) é a mesma obtida em [Eweda, 1994; Sayed, 2003] para os algoritmos RLS e LMS. Isto significa que, como na comparação entre o RLS e o LMS, há situações em que o SWA e o SLNA apresentam uma capacidade de tracking superior à do CMA e vice-versa. A diferença no tracking desses algoritmos depende da matriz $\mathbf{Q}$, sendo usual considerar os seguintes exemplos [Eweda, 1994; Sayed, 2003]:

(i) $\mathbf{Q}=\sigma_{q}^{2} \mathbf{I}$, em que o desempenho do CMA é similar ao do SWA e do SNLA;

(ii) Q é um múltiplo de $\mathbf{R}$, em que o CMA é superior; e

(iii) Q é um múltiplo de $\mathbf{R}^{-1}$, em que o SWA e o SNLA são superiores.

Considerando agora o MSE num ambiente estacionário e assumindo $\varsigma=1-\lambda$, obtém-se

$$
\frac{\zeta^{\mathrm{SNLA}}}{\zeta^{\mathrm{CMA}}}=\frac{\zeta^{\mathrm{SWA}}}{\zeta^{\mathrm{CMA}}}=\frac{M(1-\lambda)}{\mu \gamma \operatorname{Tr}(\mathbf{R})} .
$$

Os parâmetros de adaptação do CMA e SWA ou SNLA podem então ser ajustados para que esses algoritmos atinjam o mesmo MSE em regime. Seja $\sigma_{u}^{2}$ a potência do sinal de entrada. De (3.46), com $\operatorname{Tr}(\mathbf{R})=M \sigma_{u}^{2}$, chega-se a

$$
\mu=\frac{1-\lambda}{\gamma \sigma_{u}^{2}}
$$




\subsection{O problema da rotação de fase}

Um problema comum da equalização autodidata baseada no critério do Módulo Constante é a rotação de fase aleatória, que deve ser compensada antes do decisor [Heidari \& NasiriKenari, 2000; Szczecinski \& Gei, 2002]. A literatura contém vários algoritmos autodidatas para correção da fase (veja, e.g., [Chen et al., 1996; Georghiades, 1997; Oh \& Stapleton, 1997; Cartwright, 1999; Heidari \& Nasiri-Kenari, 2000]). A comparação desses algoritmos foge do escopo deste trabalho. Desta forma, assume-se por simplicidade, o Phase Tracking Algorithm (PTA) modificado [Szczecinski \& Gei, 2002; Proakis, 1996], cuja equação de atualização é dada por

$$
\theta(n+1)=\theta(n)+\mu_{\theta} \operatorname{Im}\left(y_{\theta}(n) \hat{a}^{*}\left(n-\tau_{d}\right)\right)
$$

sendo $\mu_{\theta}$ o passo de adaptação, $y_{\theta}(n)=y(n) e^{-j \theta(n)}$ a saída do equalizador com a fase corrigida e $\operatorname{Im}(x)$ a parte imaginária de $x$. Esse algoritmo faz com que a constelação de saída tenha a mesma forma da originalmente transmitida. Devido à simetria circular, a forma da maioria das constelações complexas é insensível a rotações múltiplas de $\pi / 2$. Conseqüentemente, o PTA não corrige rotações desse tipo. Para se evitar rotações múltiplas de $\pi / 2$, deve-se utilizar a codificação diferencial [Proakis, 1996].

Na seção seguinte, são abordados algoritmos concorrentes para equalização autodidata, assumindo-se constelações do tipo M-QAM (M-Quadrature Amplitude Modulation). Além de tentar eliminar a ISI, esses algoritmos corrigem automaticamente rotações de fase nãomúltiplas de $\pi / 2$. Neste caso, o uso do PTA ou de outro algoritmo de correção de fase se torna desnecessário.

\subsection{Os algoritmos concorrentes}

Dentre os algoritmos autodidatas, o CMA é o mais utilizado na prática, devido à sua baixa complexidade computacional [Ding \& Li, 2001]. Em geral, após a convergência, o CMA é chaveado para o algoritmo de Decisão Direta (DD - Decision Direct) a fim de minimizar o MSE atingido em regime [Macchi \& Eweda, 1984]. No entanto, para o funcionamento adequado desse esquema, o MSE em regime deve ser suficientemente pequeno, o que nem sempre ocorre [De Castro et al., 2001]. 
Neste contexto, De Castro et al. (2001) propuseram uma forma concorrente de operar o algoritmo de Decisão Direta com o CMA para constelações do tipo $M$-QAM. Para evitar propagação de erros devido às decisões incorretas, a adaptação do algoritmo DD é feita após a do CMA. Seus coeficientes são ajustados apenas se a adaptação do CMA tiver sido considerada correta [De Castro et al., 2001; Chen, 2003]. Com um aumento moderado na complexidade computacional, o CMA+DD apresenta uma grande melhora de desempenho em relação ao CMA.

Inspirando-se nessa idéia, Chen (2003) propôs uma modificação nesse esquema, substituindo o algoritmo de Decisão Direta por outro com Decisão Direta Suave (SDD - Soft Decision-Direct), proposto originalmente em [Karaoguz \& Adarlan, 1992] para 4-QAM e estendido em [Chen et al., 1995] para M-QAM. O CMA+SDD, também chamado de bootstrap maximum a posteriori probability [Chen et al., 2004a], possui uma complexidade computacional menor e uma taxa de convergência mais rápida do que o CMA+DD. Esses algoritmos ainda têm capacidade de corrigir rotações de fase não-múltiplas de $\pi / 2$ em constelações do tipo $M$-QAM. Na Figura 3.5, é mostrado um diagrama de blocos com as relações entre os algoritmos. A linha tracejada indica que o CMA+SDD foi inspirado no CMA+DD.

No Apêndice D, o CMA+SDD é apresentado em detalhe. Cabe observar que é possível combinar outros algoritmos de forma concorrente, obtendo-se por exemplo o AC-CMA+SDD ou o SWA+SDD. Com um aumento na complexidade computacional, esses algoritmos podem apresentar uma velocidade de convergência maior que a do CMA+SDD.

\subsection{Resultados experimentais}

Nesta seção, apresentam-se os resultados de simulações com os algoritmos autodidatas do gradiente estocástico. Inicialmente, eles são comparados aos algoritmos do gradiente determinístico que utilizam estimativas em bloco. A habilidade para evitar mínimos locais é verificada, utilizando-se canais IIR perfeitamente equalizáveis. Observa-se também o comportamento dos algoritmos em canais FIR que possuem zeros próximos ou sobre a circunferência unitária na ausência de ruído. Em seguida, considera-se um canal FIR variante no tempo a fim de verificar a capacidade dos algoritmos de acompanhar as variações do canal. Um canal de rádio digital é utilizado e os algoritmos são implementados com sobre-amostragem. Fi- 


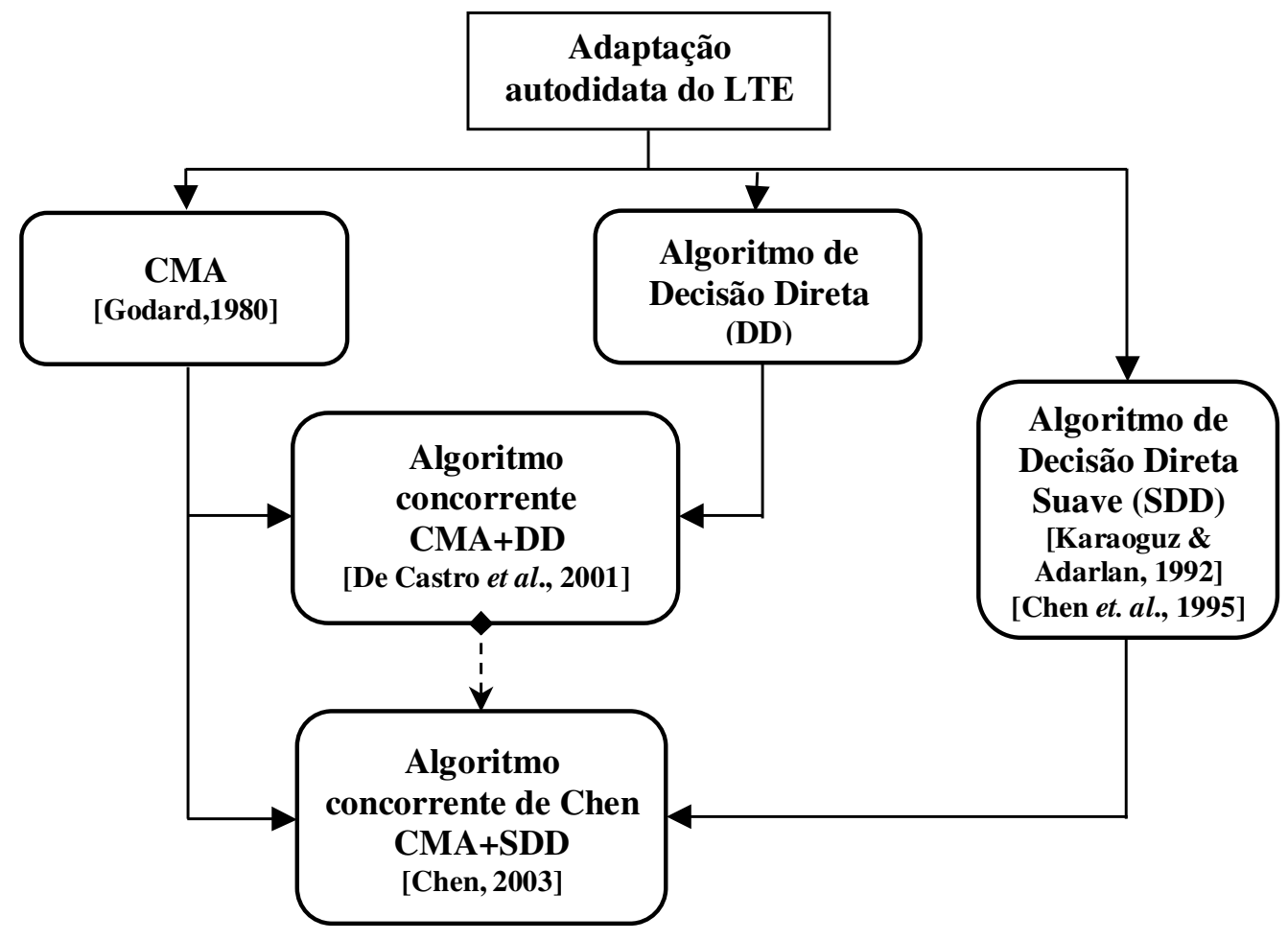

Figura 3.5: Relações entre os algoritmos concorrentes para adaptação autodidata do LTE.

nalmente, consideram-se os algoritmos de forma concorrente e uma simulação para verificar a validade da análise de tracking é apresentada.

Como medida de desempenho, utiliza-se a interferência intersimbólica (ISI) residual definida na Equação (2.4, página 34). Nas simulações, os algoritmos foram ajustados para atingir o mesmo erro quadrático médio (MSE) em regime. Para isso, o passo de adaptação do CMA foi escolhido em função do fator de esquecimento do SWA, considerando-se a Equação (3.47). No caso do AC-CMA, como ainda não foi feita uma análise de convergência e estabilidade, adotou-se a mesma sistemática de escolha dos parâmetros do AAFA. Resultados de simulações sugerem que, escolhendo $m_{1} \approx 0,15$ e $\kappa \approx 100$, deve-se variar o parâmetro $\mu$ no intervalo ]0,1[ para obter um desempenho adequado do algoritmo. Para fazer com que ele atinja o mesmo MSE em regime dos demais, esse parâmetro foi ajustado experimentalmente como $\mu_{\mathrm{AC}-\mathrm{CMA}}=k \mu_{\mathrm{CMA}}$, sendo $k \in\left[10^{2}, 10^{3}\right]$.

Se não for dito explicitamente, a inicialização dos algoritmos é feita com um vetor de $M$ elementos do tipo $\mathbf{w}(0)=\left[\begin{array}{lllllll}0 & \cdots & 0 & 1 & 0 & \cdots & 0\end{array}\right]^{T}$, sendo que o elemento não-nulo é inserido na posição central do vetor. 


\subsubsection{Comparação com os algoritmos do gradiente determinístico}

Considerando as mesmas condições de simulação da Seção 2.8, página 63 , ou seja, canal $\mathrm{H}_{2}$ definido em (2.143) e um equalizador com $M=16$ coeficientes inicializados da forma (2.144), obtêm-se os resultados da Figura 3.6. Os algoritmos foram ajustados para atingir o mesmo patamar de ISI residual. Às custas de uma complexidade computacional que impede sua utilização em inúmeras aplicações práticas, os algoritmos do gradiente determinístico SDCMA e SD-SWA apresentam uma velocidade de convergência bem maior que os do gradiente estocástico. O SWA apresenta uma velocidade de convergência média maior que a do CMA, necessitando aproximadamente de um quarto das iterações deste algoritmo para atingir o patamar final de $-19 \mathrm{~dB}$ de ISI residual. É importante observar que para $N_{b}<780$, os algoritmos do gradiente determinístico apresentam um desempenho pior (em termos de ISI residual) que o dos algoritmos do gradiente estocástico. Esse desempenho só será melhor ao se considerar $N_{b}>780$, como pode ser observado na Figura 2.3. No caso dos algoritmos do gradiente estocástico, os parâmetros de adaptação $(\mu, \lambda$ etc.) são os responsáveis pela velocidade de convergência e pelo patamar de ISI residual atingido.

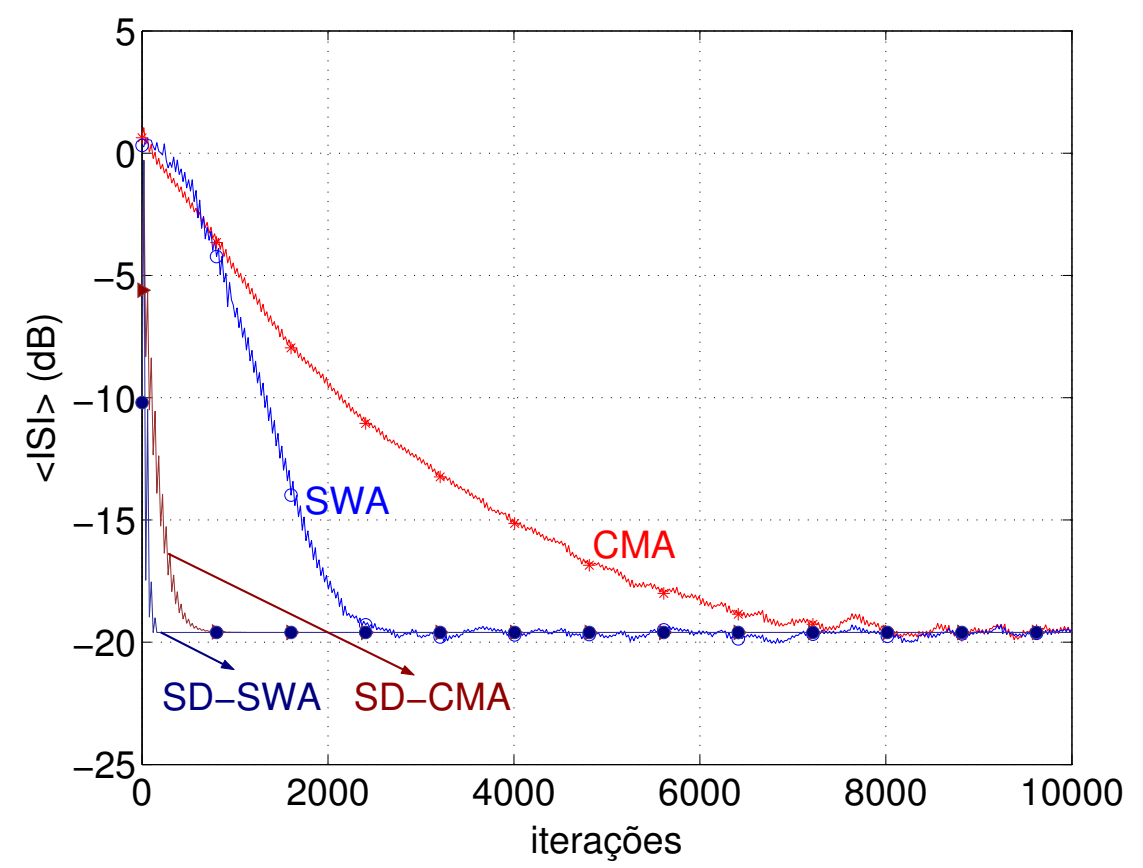

Figura 3.6: ISI residual média (50 realizações); canal $\mathrm{H}_{2}, \mathrm{SNR}=30 \mathrm{~dB}, M=16$ e 6-PAM; $\operatorname{SD-CMA}\left(\mu=10^{-4}, N_{b}=780\right)$; $\operatorname{SD}-\operatorname{SWA}\left(\beta_{S W}=0,1, N_{b}=780\right) ; \operatorname{CMA}\left(\mu=1,46 \times 10^{-5}\right)$; e SWA $(\lambda=0,9975, \delta=0,0025)$. 


\subsubsection{Canais IIR}

Os canais IIR considerados têm as seguintes funções de transferência [Ding \& Li, 2001]

$$
\mathrm{H}_{k}(z)=\frac{1}{1-p_{k} z^{-1}}
$$

para $k=1,3, p_{1}=-0,6$ e $p_{3}=-0,2$. Para o canal $\mathrm{H}_{1}$ sem ruído e sinalização 2-PAM, a função custo de Godard está esquematizada na Figura 2.1, página 43. Neste caso, há dois mínimos locais, dois mínimos globais e um máximo local. Na Figura 3.7, são mostradas as curvas de nível e as trajetórias do SWA, CMA e AC-CMA, inicializados em três pontos distintos. Inicializando em $\mathbf{w}(0)=\left[\begin{array}{ll}0 & 1\end{array}\right]^{T}$, os algoritmos apresentam comportamentos semelhantes, convergindo para um mínimo global. Para $\mathbf{w}(0)=\left[\begin{array}{ll}-0,4 & 0,05\end{array}\right]^{T}$, que fica próximo de um mínimo local, o AC-CMA escapa desse mínimo, atingindo um mínimo global, enquanto o SWA e o CMA ficam "encalhados" no mínimo local. Para $\mathbf{w}(0)=[0,2-0,4]^{T}$, o AC-CMA atinge um dos mínimos globais, atravessando a bacia de atração de um mínimo local, enquanto o CMA e o SWA convergem para o outro mínimo global. Neste caso, o AC-CMA segue o "caminho das pedras", enquanto os outros vão diretamente para o vale. Evitando com sucesso o mínimo local, o AC-CMA apresenta comparativamente uma baixa velocidade de convergência, como é mostrado na Figura 3.8.

Considerando o canal $\mathrm{H}_{3}$, os mínimos locais da função custo de Godard tornam-se mais pronunciados. Na Figura 3.9, são mostradas as curvas de nível dessa função e as trajetórias dos algoritmos inicializados nos pontos indicados. Neste caso, o AC-CMA apresenta um comportamento semelhante ao dos outros algoritmos, sendo atraído para o mínimo local. Esses resultados sugerem que o AC-CMA apresenta uma certa habilidade para escapar de mínimos locais suaves. Cabe observar que nem sempre a convergência para um mínimo local é indesejável. No exemplo considerado, o fato do mínimo local ser pronunciado pode possibilitar a correta recuperação do sinal transmitido [Regalia, 1998]. 


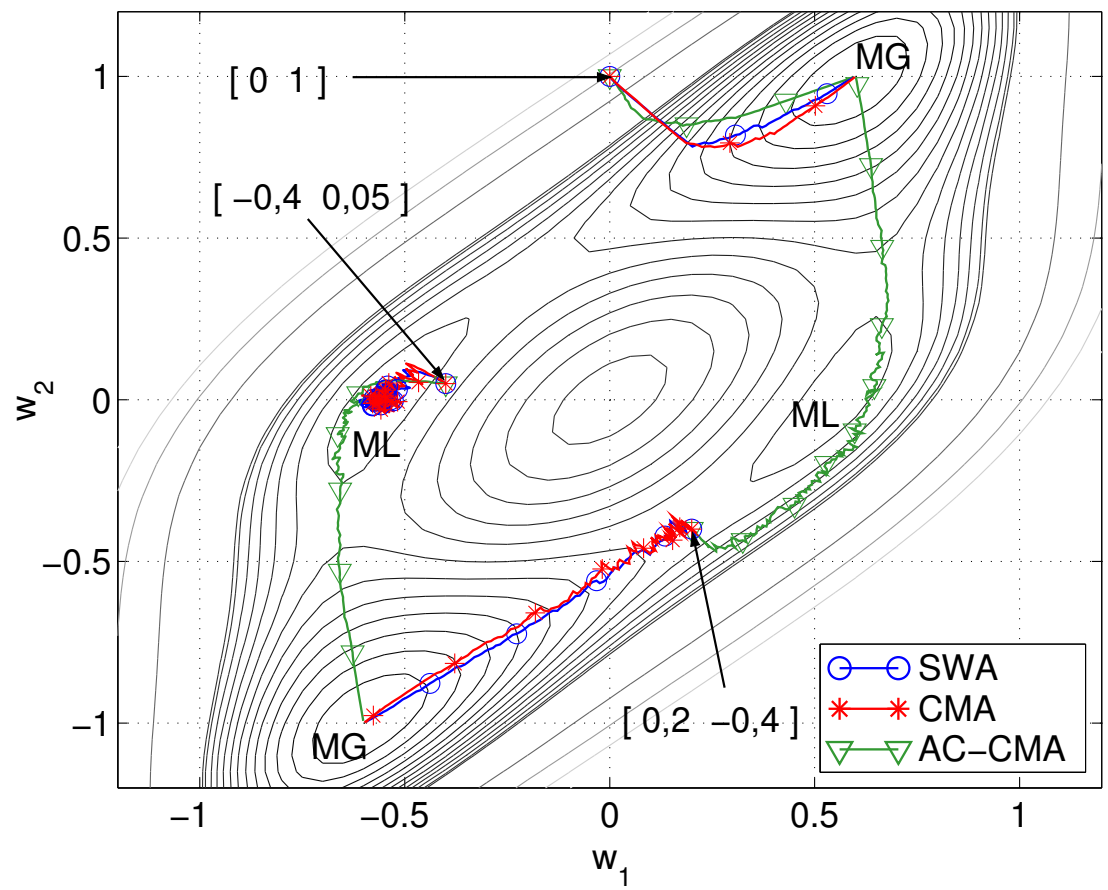

Figura 3.7: Curvas de nível da função custo de Godard para 2-PAM e canal $\mathrm{H}_{1}$ e trajetórias do SWA $(\lambda=0,995, \delta=0,005)$, CMA $(\mu=0,0016)$ e AC-CMA $\left(\mu=0,16, m_{1}=0,15, \kappa=100\right)$ inicializados nos pontos indicados; MG indica mínimo global e ML mínimo local.

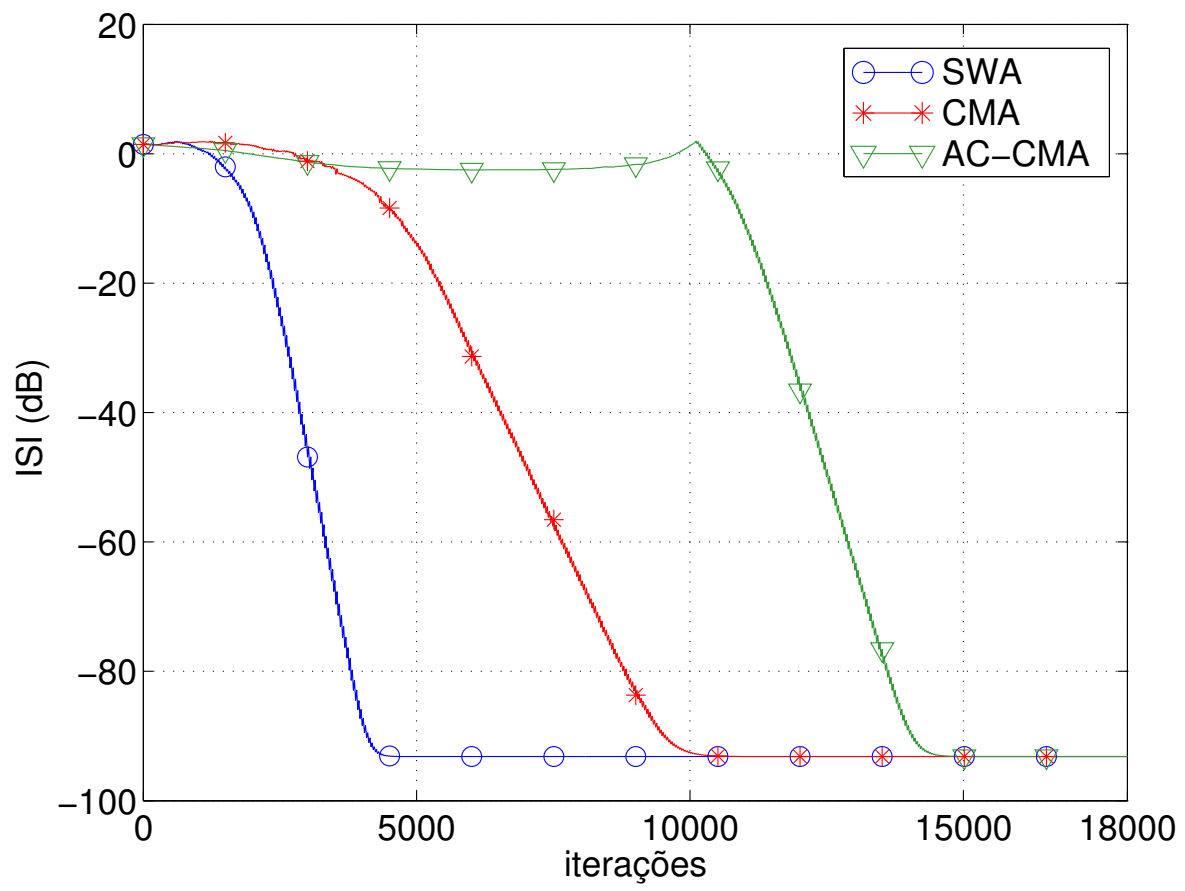

Figura 3.8: ISI residual do SWA $(\lambda=0,995, \delta=0,005)$, CMA $(\mu=0,0016)$ e AC-CMA $\left(\mu=0,16, m_{1}=0,15, \kappa=100\right)$, inicializados em $\mathbf{w}(0)=\left[\begin{array}{ll}0,2 & -0,4\end{array}\right]^{T}$; canal $\mathrm{H}_{1}, M=2$ e 2-PAM. 


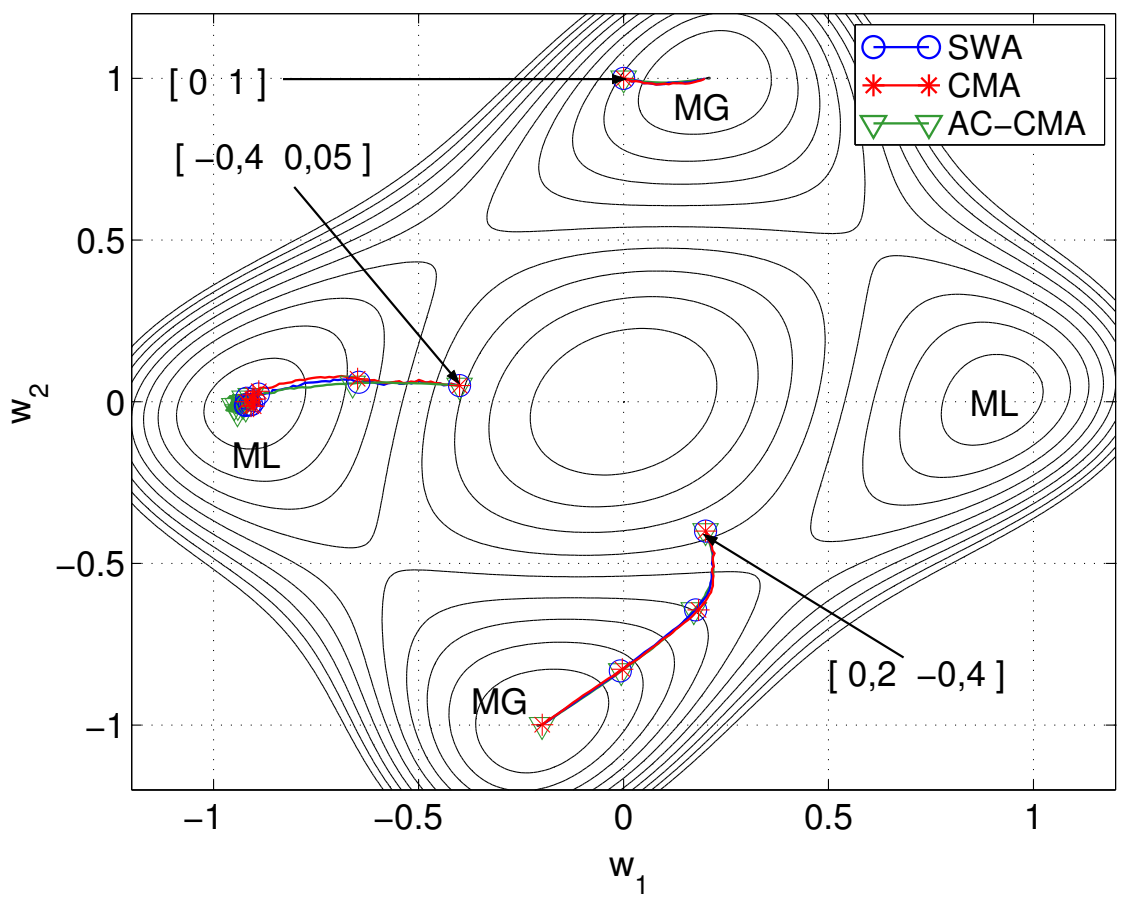

Figura 3.9: Curvas de nível da função custo de Godard para 2=PAM e canal $\mathrm{H}_{2}$ e trajetórias do SWA $(\lambda=0,995, \delta=0,005)$, CMA $(\mu=0,0024)$ e AC-CMA $\left(\mu=0,24, m_{1}=0,15, \kappa=100\right)$, inicializados nos pontos indicados; MG indica mínimo global e ML mínimo local.

\subsubsection{Canais FIR invariantes no tempo}

As funções de transferência dos canais utilizados nas simulações desta seção são apresentadas na Tabela 3.6. Os canais foram normalizados para que a potência do sinal de saída fosse igual à do sinal de entrada, tornando o ajuste dos parâmetros dos algoritmos independente do canal.

\begin{tabular}{|l|}
\hline $\mathrm{H}_{4}(z)=\left(1+2 z^{-1}+z^{-2}\right) / \sqrt{6}$ \\
Zeros: $\{-1 ;-1\}$ \\
\hline $\mathrm{H}_{5}(z)=\left(1+2 z^{-1}+0,96 z^{-2}\right) / \sqrt{5,9216}$ \\
Zeros: $\{-1,2 ;-0,8\}$ \\
\hline $\mathrm{H}_{6}(z)=\left(1+1,6 z^{-1}+z^{-2}\right) / \sqrt{4,56}$ \\
Zeros: $\left\{e^{ \pm j \theta} ; \theta=\pi / 4,882\right\}$ \\
\hline $\mathrm{H}_{7}(z)=\left(1+1,9 z^{-1}+1,2 z^{-2}\right) / \sqrt{6,05}$ \\
Zeros: $\left\{1,0954 e^{ \pm j \theta} ; \theta=5 \pi / 6\right\}$ \\
\hline
\end{tabular}

Tabela 3.6: Modelos dos canais usados nas simulações da Seção 3.8.3. 
Na Figura 3.10, são mostradas curvas de ISI residual média do SWA, CMA e AC-CMA para os canais da Tabela 3.6 na ausência de ruído e sinalização 4-QAM. Para os canais $\mathrm{H}_{4}$ e $\mathrm{H}_{5}$, que possuem zeros reais sobre e próximo da circunferência unitária respectivamente, o AC-CMA apresenta um desempenho intermediário, com uma velocidade de convergência maior que a do CMA e menor que a do SWA conforme mostrado nas figuras 3.10-a e 3.10-b. Para os canais $\mathrm{H}_{6}$ e $\mathrm{H}_{7}$, que possuem zeros complexos sobre e próximo da circunferência unitária respectivamente, o AC-CMA apresenta um comportamento mais próximo do SWA que o CMA, sendo que este último converge lentamente como pode ser observado nas figuras 3.10-c e 3.10-d.
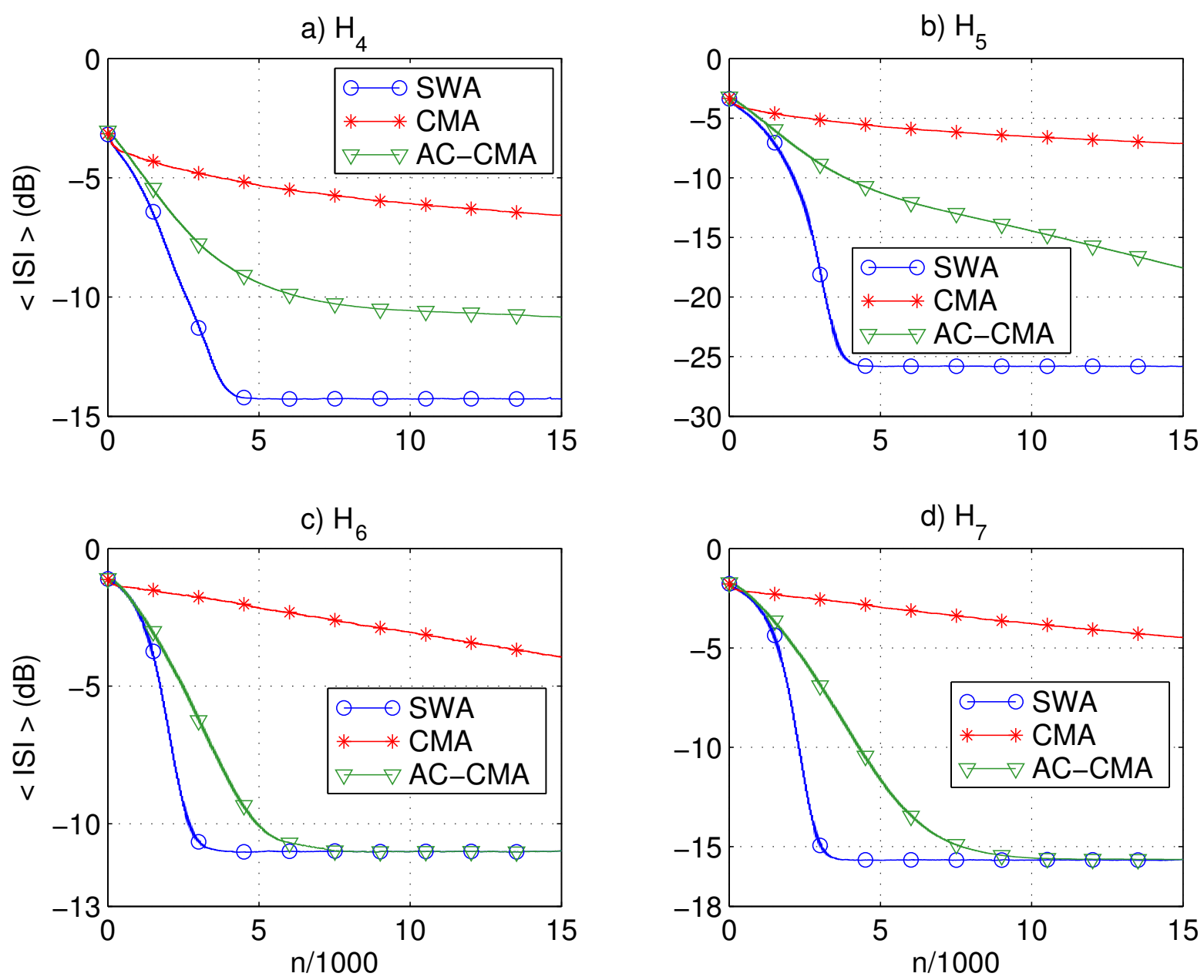

Figura 3.10: ISI residual média ao longo das iterações do SWA $(\lambda=0,9975, \delta=0,0025)$, CMA $\left(\mu=6,2 \times 10^{-4}\right)$ e AC-CMA $\left(\mu=0,062, m_{1}=0,15, \kappa=100\right)$ para 4-QAM, $M=27$, média de 50 realizações e canais a) $\mathrm{H}_{4}$, b) $\mathrm{H}_{5}$, c) $\mathrm{H}_{6}$ e d) $\mathrm{H}_{7}$. 


\subsubsection{Canal FIR variante no tempo}

Os coeficientes do canal variante no tempo são mostrados na Figura 3.11. Eles foram gerados a partir da filtragem do ruído branco gaussiano por um filtro de Butterworth de segunda ordem, projetado para simular uma taxa de desvanecimento de 0,1 Hz [Shimamura \& Cowan, 1997; Cowan \& Semnani, 1998]. Além disso, foi considerada uma normalização a fim de que a potência do sinal de saída do canal fosse igual à potência de seu sinal de entrada, facilitando o ajuste dos parâmetros dos algoritmos.



Figura 3.11: Coeficientes do canal $\mathrm{H}_{8}$ ao longo das iterações.

Na Figura 3.12, são considerados os erros na recuperação do sinal transmitido para o CMA, SWA e AC-CMA, sinalização 2-PAM e uma relação sinal-ruído de $20 \mathrm{~dB}$. O valor absoluto das raízes do polinômio $h_{0}(n) x^{2}+h_{1}(n) x+h_{2}(n)$ ao longo das iterações é mostrado na Figura 3.12-d. Os erros em seqüência que ocorrem próximos das iterações 5000, 11000, 22000, 27000 e 37000 são associados a nulos espectrais (o valor absoluto igual a 1 é indicado por uma linha reta). O AC-CMA apresenta uma recuperação mais rápida que o CMA e possui um comportamento próximo ao do SWA. Particularmente, o AC-CMA e o SWA não sofrem com o nulo espectral próximo da iteração 22000, enquanto o CMA apresenta erros em seqüência nesta situação. As correspondentes curvas de ISI residual são mostradas na Figura 3.12-e. Cabe observar que com a normalização do canal, o CMA se comporta como 
um algoritmo normalizado. Na ausência da normalização, para um passo de adaptação fixo, o CMA apresenta um desempenho inferior ao apresentado na Figura 3.12-a. Uma solução seria considerar um passo de adaptação variável que dependesse da potência do sinal de entrada. No entanto, fazer uma boa estimativa da potência só é viável em canais com variações lentas.
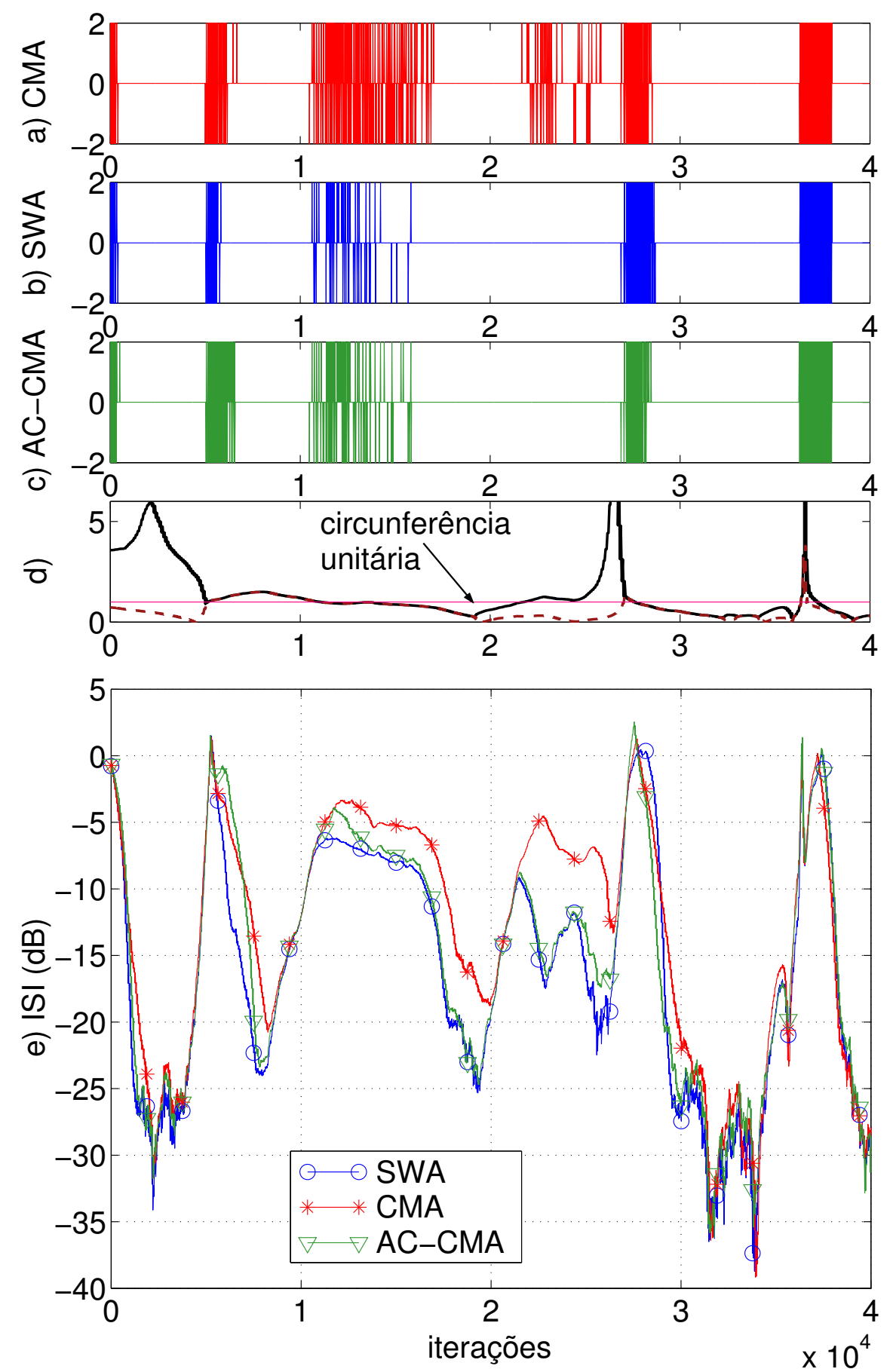

Figura 3.12: a), b) e c) Erros na recuperação do sinal utilizando respectivamente o CMA ( $\mu=$ $0,0025)$, o SWA $(\lambda=0,995, \delta=0,005)$ e o AC-CMA $\left(\mu=0,25, m_{1}=0,15, \kappa=100\right)$; d)Módulos dos zeros do canal $\mathrm{H}_{8}$ ao longo das iterações; e) ISI residual; $\mathrm{SNR}=20 \mathrm{~dB}, M=11$ e 2-PAM. 


\subsubsection{Canal de rádio digital}

O canal de rádio digital utilizado nas simulações foi obtido a partir do processamento de medidas de campo. Ele é caracterizado por um filtro FIR com 300 coeficientes complexos, obtidos da sobre-amostragem $(L=2)$ de um modelo de tempo contínuo [SPIB, 2001, chan9.mat]. Sua resposta em freqüência e gráficos dos coeficientes estão mostrados na Figura 3.13. Os nulos espectrais pronunciados em sua resposta em freqüência tornam sua equalização difícil, sendo necessária a utilização da técnica de sobre-amostragem, revisitada no Apêndice A.
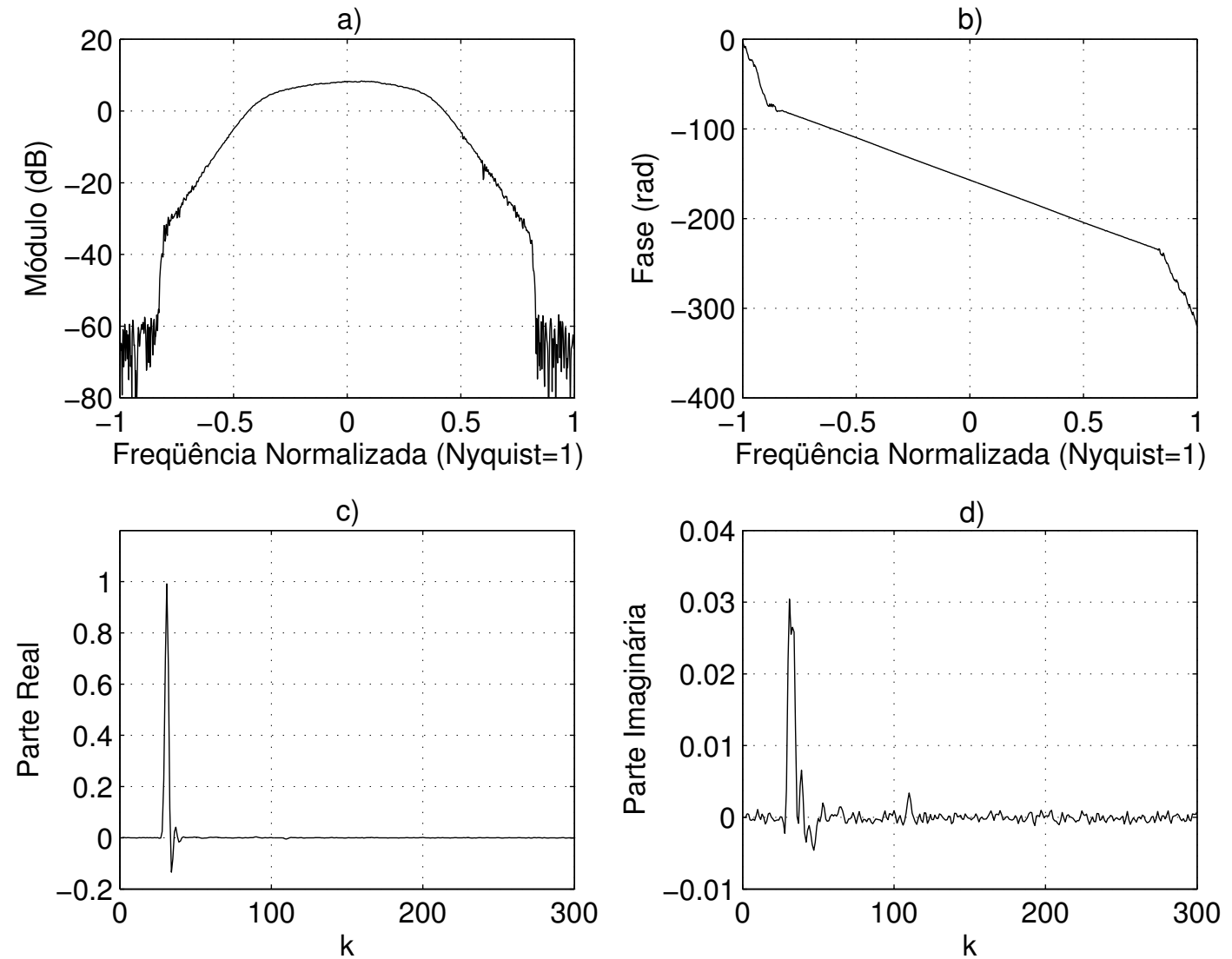

Figura 3.13: a) e b) Resposta em freqüência, c) Parte Real e d) Parte Imaginária dos coeficientes do canal $\mathrm{H}_{9}$ [SPIB, 2001, chan9.mat].

Na Figura 3.14, são mostradas curvas de ISI residual média considerando o SWA, o CMA e o AC-CMA para o treinamento de um equalizador com $M=6$ coeficientes, sobreamostrado por $L=2$. O AC-CMA e o SWA apresentam desempenhos próximos e uma velocidade de convergência superior à do CMA. 


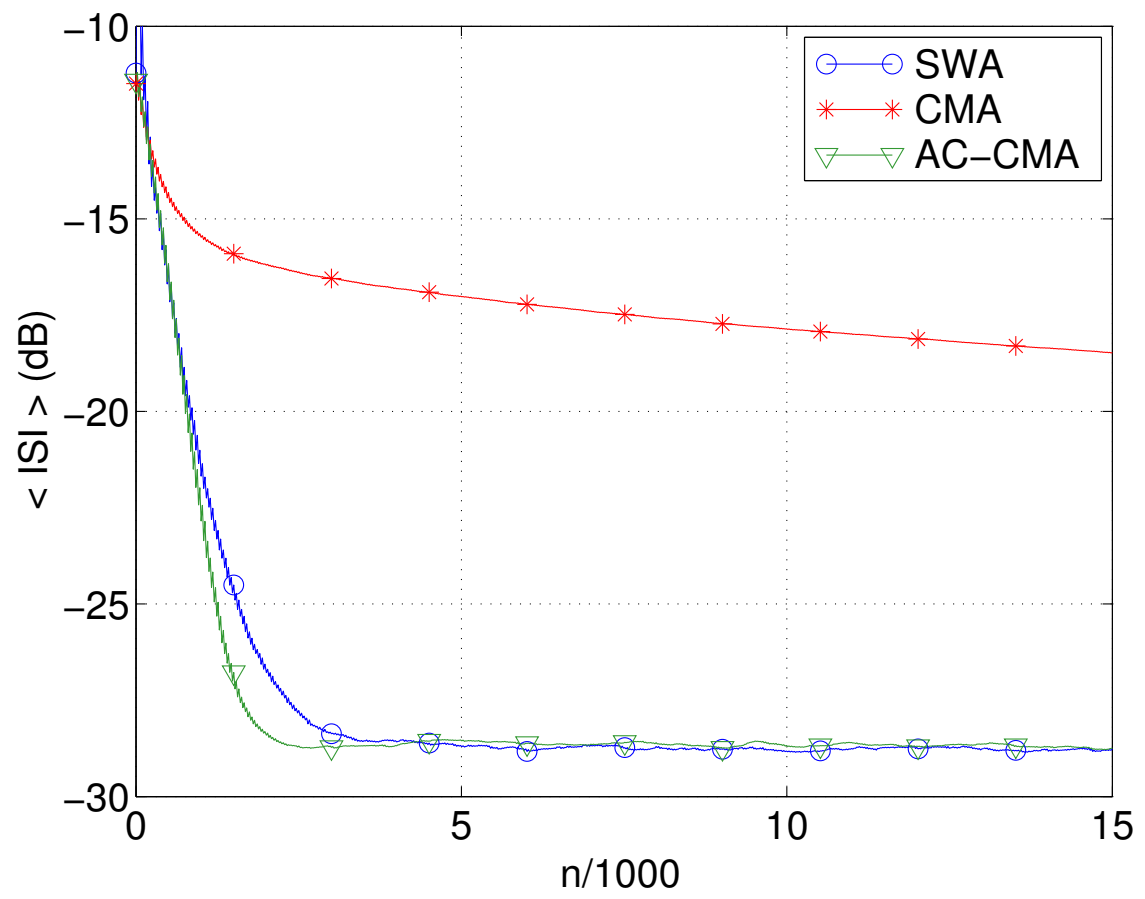

Figura 3.14: ISI residual média (50 realizações); canal $\mathrm{H}_{9}$, $\mathrm{SNR}=25 \mathrm{~dB}, 4$-QAM, $M=6, L=2$; SWA $(\lambda=0,999, \delta=0,1)$; CMA $\left(\mu=2,5 \times 10^{-4}\right)$; e AC-CMA $\left(\mu=0,1, m_{1}=0,15, \kappa=100\right)$.

Na Figura 3.15, são mostradas as constelações de saída de uma realização do equalizador, considerando as $10^{3}$ últimas iterações dos algoritmos. Passando o sinal de saída por um decisor, verifica-se que o sinal transmitido é recuperado adequadamente mesmo com o CMA que apresentou uma convergência lenta.

a) SWA

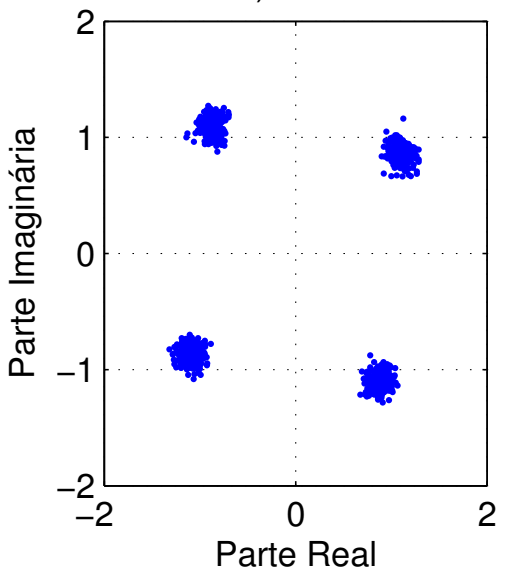

b) $\mathrm{CMA}$

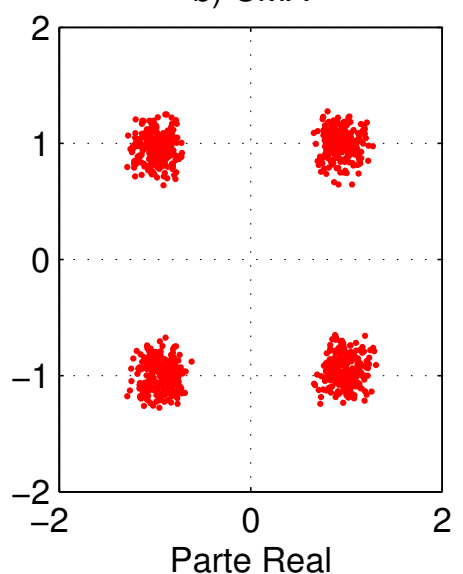

c) $\mathrm{AC}-\mathrm{CMA}$

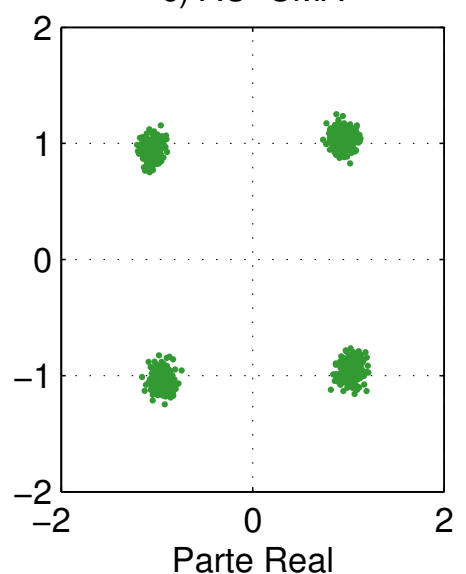

Figura 3.15: Sinal de saída do equalizador entre as iterações 14000 e $15000 ; M=6, L=2$, canal $\mathrm{H}_{9}, \mathrm{SNR}=25 \mathrm{~dB}$ e 4-QAM; a) SWA $(\lambda=0,999, \delta=0,1)$; b) CMA $\left(\mu=2,5 \times 10^{-4}\right)$; c) AC-CMA $\left(\mu=0,1, m_{1}=0,15, \kappa=100\right)$. 
Observando a Figura 3.14, poderia ser concluído que algoritmos mais rápidos que o CMA, como o AC-CMA ou o SWA, têm um papel fundamental na equalização. No entanto, mesmo convergindo lentamente, o CMA consegue recuperar de maneira adequada o sinal. Além disso, o desempenho do SWA está intimamente ligado à inicialização da matriz de autocorrelação inversa. Dependo do valor de $\delta$, ele pode apresentar uma convergência inicial lenta ou pode divergir devido a problemas numéricos. Diante dessas observações, o CMA apresenta um bom compromisso entre comportamento eficiente e complexidade computacional, quando utilizado num equalizador sobre-amostrado. Para isso, deve-se escolher adequadamente o passo de adaptação, o que pode ser um problema em muitas situações. Neste contexto, o AC-CMA é uma boa alternativa, dado que não depende da inicialização da matriz de autocorrelação. Além disso, a escolha do seu passo de adaptação parece ser menos crítica que no caso do CMA. Ele ainda possui uma complexidade computacional que fica entre a do CMA e a do SWA, mantendo a proporcionalidade a $M$ operações por iteração.

\subsubsection{Algoritmos concorrentes}

A resposta em freqüência do canal $\mathrm{H}_{10}$ [Chen, 2003; Chen et al., 2004a; Chen \& Chng, 2004b], usado nas simulações com os algoritmos concorrentes, é apresentada na Figura 3.16 e seus coeficientes mostrados na Tabela 3.7.
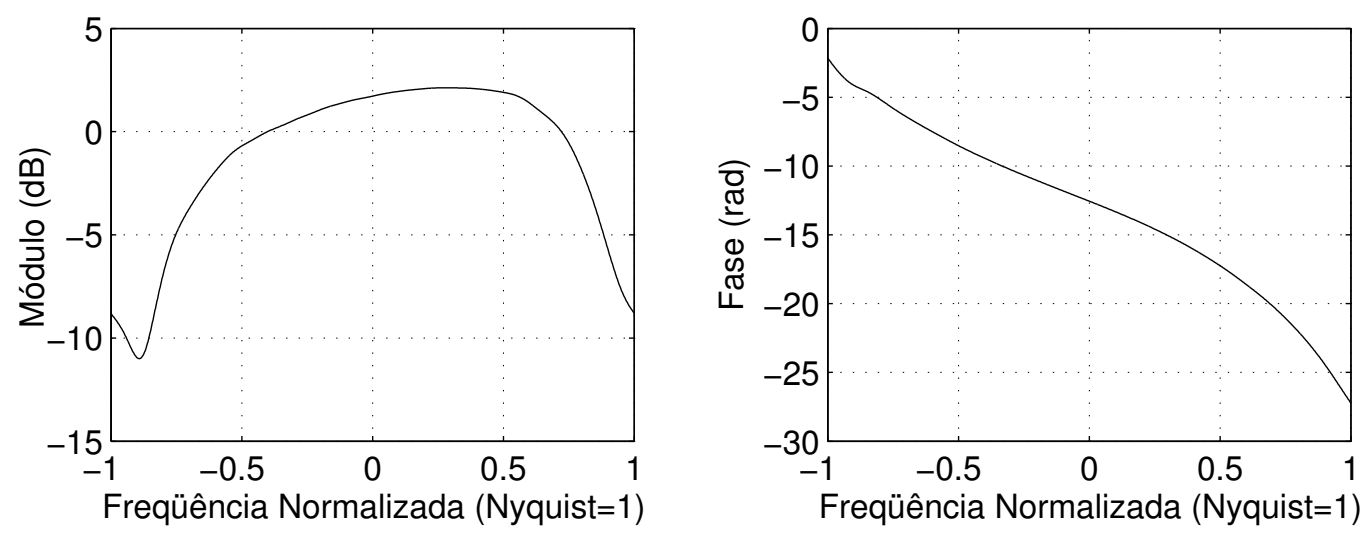

Figura 3.16: Resposta em freqüência do canal $\mathrm{H}_{10}$. 


\begin{tabular}{|c|c|c||c|c|c|}
\hline Coef. № & Parte Real & Parte Imag. & Coef. № & Parte Real & Parte Imag. \\
\hline 0 & 0,0145 & $-0,0006$ & 11 & 0,0294 & $-0,0049$ \\
\hline 1 & 0,0750 & 0,0176 & 12 & $-0,0181$ & 0,0032 \\
\hline 2 & 0,3951 & 0,0033 & 13 & 0,0091 & 0,0003 \\
\hline 3 & 0,7491 & $-0,1718$ & 14 & $-0,0038$ & $-0,0023$ \\
\hline 4 & 0,1951 & 0,0972 & 15 & 0,0019 & 0,0027 \\
\hline 5 & $-0,2856$ & 0,1896 & 16 & $-0,0018$ & $-0,0014$ \\
\hline 6 & 0,0575 & $-0,2096$ & 17 & 0,0006 & 0,0003 \\
\hline 7 & 0,0655 & 0,1139 & 18 & 0,0005 & 0,0000 \\
\hline 8 & $-0,0825$ & $-0,0424$ & 19 & $-0,0008$ & $-0,0001$ \\
\hline 9 & 0,0623 & 0,0085 & 20 & 0,0000 & $-0,0002$ \\
\hline 10 & $-0,0438$ & 0,0034 & 21 & 0,0001 & 0,0006 \\
\hline
\end{tabular}

Tabela 3.7: Resposta ao pulso unitário do canal $\mathrm{H}_{10}$.

Na Figura 3.17, são mostradas curvas de ISI residual média para o SWA, CMA, AC-CMA e suas versões concorrentes com o algoritmo SDD [Chen et al., 1995]. Além disso, para efeito de comparação, o algoritmo SDD também é considerado de forma não-concorrente. Assume-se a transmissão de um sinal com 16-QAM através do canal $\mathrm{H}_{10}$ com $\mathrm{SNR}=30 \mathrm{~dB}$. Observa-se que os algoritmos concorrentes apresentam uma maior velocidade de convergência e um patamar de ISI residual inferior ao dos não-concorrentes. O AC-CMA+SDD apresenta uma velocidade de convergência maior que a do CMA+SDD e menor que a do SWA+SDD. O algoritmo SDD não-concorrente apresenta em média uma convergência bastante lenta. Na Figura 3.18, são mostradas as constelações de saída do equalizador, considerando as $10^{3}$ últimas iterações de uma realização dos algoritmos. Deve-se notar que os algoritmos concorrentes são capazes de corrigir a rotação de fase que aparece nos não-concorrentes. Esses algoritmos ainda podem ser utilizados na adaptação de equalizadores sobre-amostrados como considerado em [Chen \& Chng, 2004b]. 


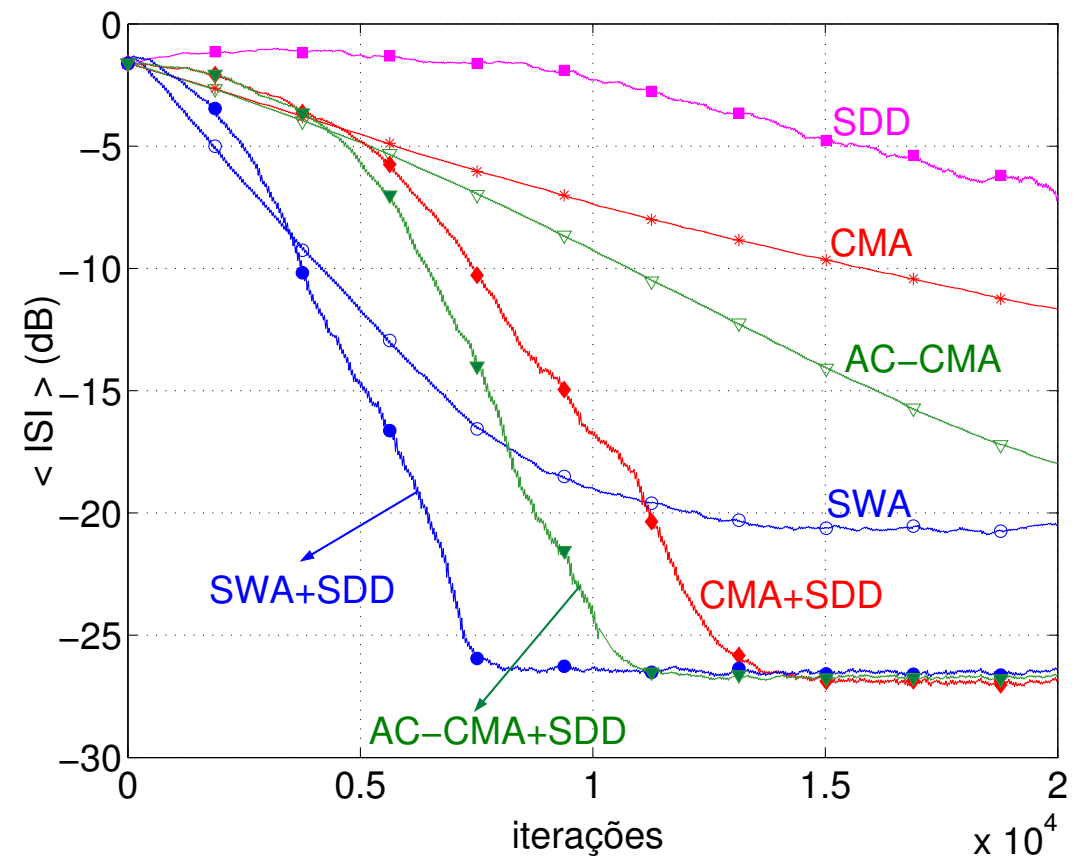

Figura 3.17: ISI residual média (50 realizações); $M=23$, canal $\mathrm{H}_{10}, \mathrm{SNR}=30 \mathrm{~dB}$ e 16-QAM; $\lambda=0,9997 ; \delta=3 \times 10^{-4} ; \mu_{\mathrm{CMA}}=1,2 \times 10^{-4} ; \mu_{\mathrm{AC}}=5 \times 10^{-3} ; m_{1}=0,15 ; \kappa=100 ; \mu_{d}=10^{-3} ; \rho=0,6$.
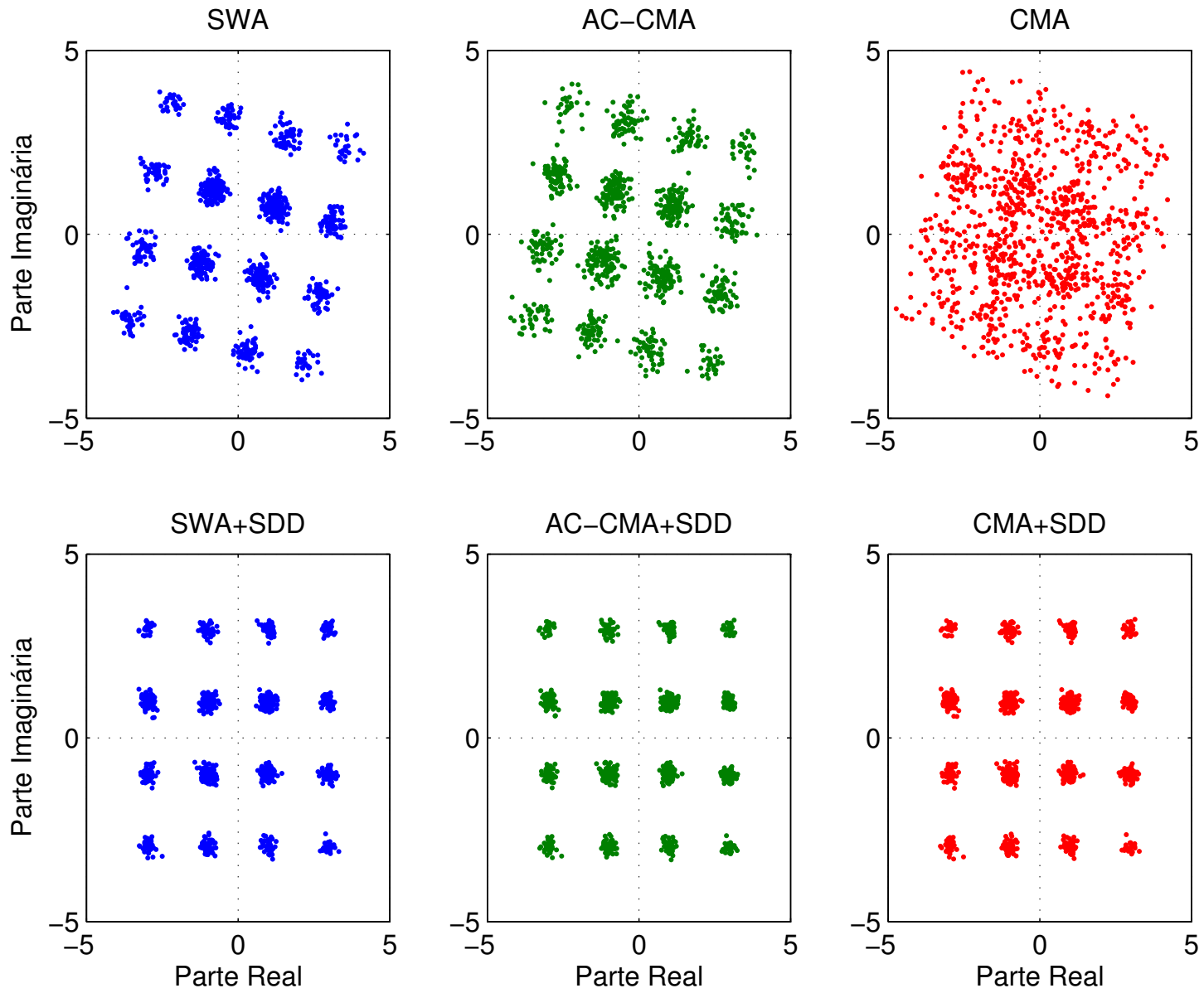

Figura 3.18: Sinal de saída do equalizador entre as iterações 19000 e 20000 dos algoritmos para $M=23$, canal $\mathrm{H}_{10}, \mathrm{SNR}=30 \mathrm{~dB}$ e 16-QAM. 


\subsubsection{Análise de tracking}

Para verificar a validade da análise de tracking do CMA, SNLA e SWA, são utilizadas as mesmas condições de simulação de [Yousef \& Sayed, 1999]. Assumem-se $\mathbf{Q}=\sigma_{q}^{2} \mathbf{I}$ e o canal $\mathrm{H}_{11}(z)=0,1+0,3 z^{-1}+z^{-2}-0,1 z^{-3}+0,5 z^{-4}+0,2 z^{-5}$. Além disso, considera-se um equalizador com $M=4$ coeficientes, sobre-amostrado por um fator $L=2, \sigma_{q}=10^{-3}$ e sinalização 6-PAM com estatísticas $E\left\{a^{6}(n)\right\}=5451,7, \mathrm{E}\left\{a^{2}(n)\right\}=11,67$ e $R_{2}^{a}=20,2$. Na Figura 3.19, é mostrado o MSE em função dos parâmetros de adaptação, considerando resultados teóricos e experimentais para o CMA, SNLA e SWA.

a) CMA

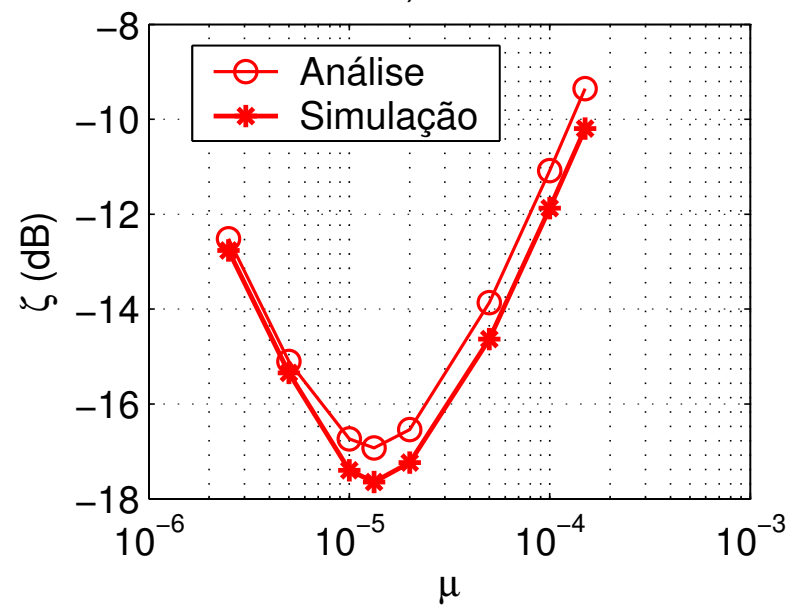

b) SNLA

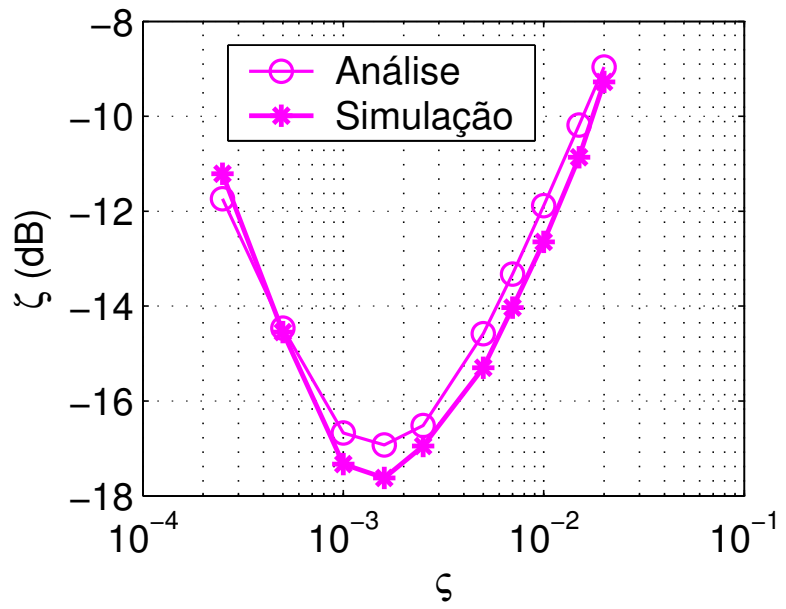

c) SWA

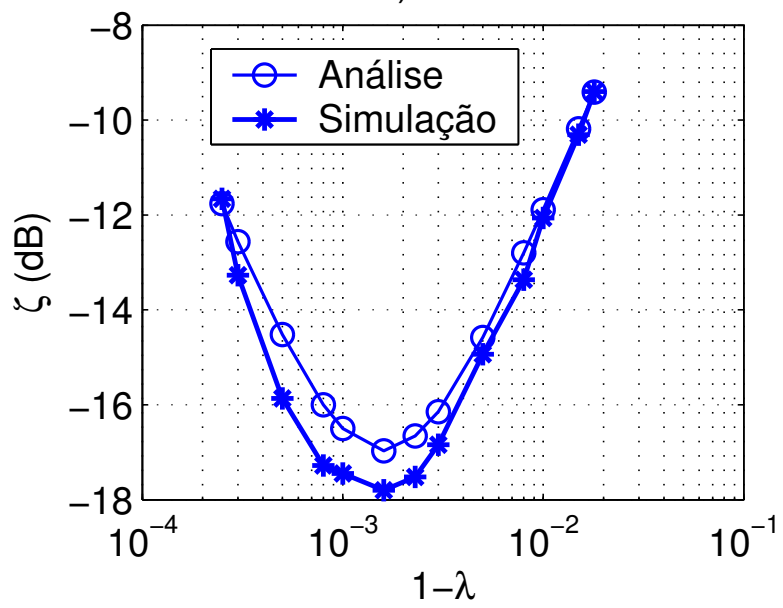

Figura 3.19: Curvas com resultados analíticos e de simulação do MSE; canal $\mathrm{H}_{11}, \mathbf{Q}=10^{-6} \mathbf{I}$, 6-PAM, $M=4, L=2$ e média de 100 realizações; a) CMA; b) SNLA; e c) SWA. 
O valor do MSE experimental foi obtido como média de 100 realizações. Os resultados da simulação apresentam uma boa concordância com os da análise para todos os algoritmos considerados. O valor teórico do MSE mínimo predito pelas expressões da Tabela 3.5 é $\zeta_{o}=-16.93 \mathrm{~dB}$, o que corresponde aos seguintes parâmetros de adaptação ótimos $\mu_{o}=$ $1,3 \times 10^{-5}$ e $\varsigma_{o}=1-\lambda_{o}=1,6 \times 10^{-3}$. Os valores experimentais são $\zeta_{o}^{\mathrm{CMA}}=-17,64 \mathrm{~dB}$, $\zeta_{o}^{\mathrm{SNLA}}=-17,62 \mathrm{~dB}$ e $\zeta_{o}^{\mathrm{SWA}}=-17,80 \mathrm{~dB}$. Estes valores estão próximos dos preditos pelas expressões da Tabela 3.5. O mesmo ocorre com os parâmetros de adaptação como pode ser observado na Figura 3.19.

\subsection{Conclusões}

Neste capítulo, foi feito um estudo do comportamento de algoritmos de equalização autodidata no caso SISO. Foram considerados o CMA, o SWA e proposto um novo algoritmo denominado AC-CMA. Assumindo a operação do equalizador na taxa de transmissão de símbolos, verificou-se através de simulações que o AC-CMA apresenta um desempenho intermediário, ou seja, dependendo do canal, ele pode se comportar como o SWA ou como o CMA, tendo uma velocidade de convergência maior que o último. Além disso, foi verificado também por simulações que ele pode evitar mínimos locais suaves, que é uma característica desejável em algoritmos autodidatas. Ele ainda possui uma boa capacidade de tracking apresentando um desempenho próximo ao do SWA e melhor que o do CMA.

Ao se considerar a sobre-amostragem, dependendo do fator $L$, a diferença de comportamento desses algoritmos deixa de ser significativa. Neste caso, o CMA pode apresentar o compromisso mais favorável entre velocidade de convergência e complexidade computacional, desde que o passo de adaptação seja escolhido adequadamente. Em contrapartida, o desempenho do SWA está ligado à inicialização da matriz de autocorrelação inversa. Dependendo do valor de $\delta$, ele pode apresentar problemas de instabilidade numérica. Neste contexto, o AC-CMA é uma boa alternativa, dado que o intervalo do passo de adaptação que garante sua convergência parece ser maior que o do CMA. Além disso, ele ainda não depende da inicialização da matriz de autocorrelação.

Uma outra forma de melhorar o desempenho dos algoritmos é considerá-los de forma concorrente com o algoritmo SDD. Além de possibilitar correções de fase não-múltiplas de 
$90^{\circ}$, os algoritmos concorrentes atingem patamares de ISI residual menores. Esses algoritmos também podem ser utilizados em equalizadores sobre-amostrados, como considerado em [Chen \& Chng, 2004b].

Uma outra contribuição deste capítulo é a análise de tracking feita para algoritmos que satisfazem a relação de conservação de energia da Equação (3.24, página 82). Partindo dessa relação, considerando um ambiente com um pequeno grau de não-estacionariedade e assumindo verdadeiras as considerações S1-S7, chegou-se ao Teorema 3 que fornece uma expressão teórica para o MSE. Através de simulações, verificou-se que há uma boa concordância entre o MSE experimental e o teórico. A razão obtida entre o MSE mínimo para o SWA e o CMA é a mesma obtida entre os algoritmos RLS e LMS. Isso possibilitou uma extensão direta da comparação desses algoritmos com relação ao tracking para o caso autodidata. 


\section{Capítulo 4}

\section{Algoritmos estocásticos para equalização multicanal}

Neste capítulo, são abordados os algoritmos autodidatas do gradiente estocástico para equalização de sistemas com múltiplas entradas e múltiplas saídas (MIMO -Multiple-Input Multiple-Output). Inicialmente, a função custo de Godard para equalização espaço-temporal é revisitada. Em seguida, abordam-se os algoritmos do Módulo Constante (MU-CMA), de Shalvi-Weinstein (MU-SWA) e do Módulo Constante Acelerado (MU-AC-CMA) para o caso multiusuário. O MU-CMA, bem conhecido na literatura [Papadias \& Paulraj, 1997b,a], é revisitado, servindo de inspiração para obtenção do MU-SWA e do MU-AC-CMA. A análise de tracking do Capítulo 3 é estendida para o MU-CMA e o MU-SWA. O capítulo termina com resultados experimentais e conclusões.

A notação utilizada ao longo deste capítulo é a mesma da formulação do problema no caso MIMO, apresentada no Capítulo 1, página 23.

\subsection{A função custo de Godard no caso MIMO}

Assumindo fontes com mesmas características estatísticas, o valor de $R_{2}^{a}$ é igual para todas elas e uma extensão direta da função custo de Godard para o caso multiusuário é dada por [Papadias \& Paulraj, 1997a]

$$
\sum_{i=1}^{N_{u}} \mathrm{E}\left\{\left(\left|y_{i}(n)\right|^{2}-R_{2}^{a}\right)^{2}\right\},
$$


sendo $y_{i}(n)$ a saída do $i$-ésimo equalizador e $N_{u}$ o número de usuários. As saídas de um equalizador otimizado por essa função podem convergir para apenas um dos diferentes sinais transmitidos e geralmente convergem para o de maior potência, ficando difícil predizer qual dos sinais será recuperado [Papadias \& Paulraj, 1997a]. Para evitar que a mesma fonte seja extraída em diferentes receptores, é usual a introdução de um termo que penaliza as correlações cruzadas entre as saídas dos filtros espaciais. Desta maneira, a extensão da função custo de Godard assume a forma [Papadias \& Paulraj, 1997b,a; Castedo et al., 1997]

$$
J_{G}=\sum_{i=1}^{N_{u}}\left[\mathrm{E}\left\{\left(\left|y_{i}(n)\right|^{2}-R_{2}^{a}\right)^{2}\right\}+\varrho \sum_{j=1, j \neq i}^{N_{u}} \sum_{k=0}^{k_{1}}\left|r_{i j}(k)\right|^{2}\right]
$$

sendo $r_{i j}(k)=\mathrm{E}\left\{y_{i}(n) y_{j}^{*}(n-k)\right\}$ e $\varrho>0$ um fator de ponderação, usualmente escolhido igual a 3 ou 4 . O parâmetro $k_{1}$ é um inteiro associado ao espalhamento de atraso (delay spread) do canal. No caso de espalhamento de atraso nulo, tem-se $k_{1}=0$, mas em geral adota-se $k_{1}=K_{t}+K_{c}$, sendo $K_{t}$ um inteiro associado à diversidade temporal e $K_{c}$ o número de coeficientes de cada canal [Luo \& Chambers, 2000, 2002].

O vetor gradiente de (4.2) em relação ao usuário $i$ é dado por

$$
\nabla_{\mathbf{w}_{i}} J_{G}(n)=2(\beta-1)\left[\mathrm{E}\left\{e_{i}(n) \mathbf{u}^{*}(n)\right\}+\frac{\varrho}{2} \sum_{j=1, i \neq j}^{N_{u}} \sum_{k=0}^{k_{1}} \mathrm{E}\left\{y_{j}(n-k) \mathbf{u}^{*}(n)\right\} r_{i j}(k)\right] \text {, }
$$

sendo

$$
e_{i}(n)=\left(\left|y_{i}(n)\right|^{2}-R_{2}^{a}\right) y_{i}(n)
$$

É usual estimar a correlação cruzada $r_{i j}(k)$ com uma janela exponencial e as outras esperanças que aparecem em (4.3) através de estimativas instantâneas [Papadias \& Paulraj, 1997a; Luo \& Chambers, 2002]. Com essas estimativas, obtém-se a seguinte aproximação estocástica para o vetor gradiente [Luo \& Chambers, 2002]

$$
\widehat{\nabla}_{\mathbf{w}_{i}} J_{G}(n)=\bar{e}_{i}(n) \mathbf{u}^{*}(n),
$$

sendo

$$
\bar{e}_{i}(n)=e_{i}(n)+\epsilon_{i}(n)
$$

e

$$
\epsilon_{i}(n)=\frac{\varrho}{2} \sum_{j=1, i \neq j}^{N_{u}} \sum_{k=0}^{k_{1}} y_{j}(n-k) \hat{r}_{i j}(k)
$$


Por conveniência, reescreve-se $\epsilon_{i}(n)$ como

$$
\epsilon_{i}(n)=\frac{\varrho}{2} \hat{\mathbf{r}}_{i}^{T}(n) \mathbf{y}_{i}(n),
$$

sendo

$$
\hat{\mathbf{r}}_{i}(n)=\left[\begin{array}{llllll}
\hat{\mathbf{r}}_{i, 1}^{T} & \cdots & \hat{\mathbf{r}}_{i, i-1}^{T} & \hat{\mathbf{r}}_{i, i+1}^{T} & \cdots & \hat{\mathbf{r}}_{i, N_{u}}^{T}
\end{array}\right]^{T}
$$

e

$$
\mathbf{y}_{i}(n)=\left[\begin{array}{llllll}
\breve{\mathbf{y}}_{1}^{T}(n) & \cdots & \breve{\mathbf{y}}_{i-1}^{T}(n) & \breve{\mathbf{y}}_{i+1}^{T}(n) & \cdots & \breve{\mathbf{y}}_{N_{u}}^{T}(n)
\end{array}\right]^{T}
$$

com

$$
\hat{\mathbf{r}}_{i, j}=\left[\begin{array}{lll}
\hat{r}_{i j}(0) & \cdots & \hat{r}_{i j}\left(k_{1}\right)
\end{array}\right]^{T}
$$

e

$$
\breve{\mathbf{y}}_{j}(n)=\left[\begin{array}{lll}
y_{j}(n) & \cdots & y_{j}\left(n-k_{1}\right)
\end{array}\right]^{T}
$$

para $1 \leq j \leq N_{u}$ e $j \neq i$. Os vetores $\hat{\mathbf{r}}_{i}(n)$ e $\mathbf{y}_{i}(n)$ possuem dimensão $\left(N_{u}-1\right)\left(k_{1}+1\right)$. Utilizando essa notação, o vetor de correlações cruzadas $\hat{\mathbf{r}}_{i}(n)$ pode ser atualizado através da seguinte equação recursiva

$$
\hat{\mathbf{r}}_{i}(n)=\lambda_{r} \hat{\mathbf{r}}_{i}(n-1)+\left(1-\lambda_{r}\right) y_{i}(n) \mathbf{y}_{i}^{*}(n),
$$

sendo $0<\lambda_{r}<1$ um fator de esquecimento.

\subsection{Algoritmos para equalização espaço-temporal}

Considerando a função custo (4.2) e a estimativa do vetor gradiente de (4.5), é possível obter algoritmos do gradiente estocástico, cuja equação de atualização para o usuário $i$ é dada por

$$
\mathbf{w}_{i}(n)=\mathbf{w}_{i}(n-1)-\alpha(n) \bar{e}_{i}(n) \mathbf{u}^{*}(n)
$$

No MU-CMA [Papadias \& Paulraj, 1997b,a], o passo de adaptação é um escalar constante, $\alpha(n)=\mu$. Esse algoritmo é mostrado na Tabela 4.1. 




Tabela 4.1: Sumário do MU-CMA.

Baseando-se nas relações entre o CMA e o SWA mostradas na Figura 3.1, página 74, outro possível candidato para passo de adaptação é $\alpha(n)=\gamma^{-1} \widehat{\mathbf{R}}^{-1}(n)$. Neste caso, obtémse o algoritmo denominado MU-SWA, que pode ser interpretado como uma extensão do SWA para o caso multiusuário [Silva et al., 2004e]. Esse algoritmo é mostrado na Tabela 4.2.

Em [Luo \& Chambers, 2000, 2001], foi proposto um algoritmo do tipo quase-Newton baseado na função custo de Godard para o caso multiusuário. Às custas de uma elevada complexidade computacional, o algoritmo denominado QN-CCCMA (Quasi-Newton CrossCorrelation Constant Modulus Algorithm) apresenta uma velocidade de convergência maior que a do MU-CMA. Porém, em algumas situações, a estimativa de sua matriz hessiana pode deixar de ser positiva-definida, levando a problemas de instabilidade numérica [Luo \& Chambers, 2000]. Além disso, sua complexidade computacional é maior que a do MU-SWA, podendo apresentar uma capacidade de tracking inferior à desse algoritmo [Silva et al., 2004e]. 


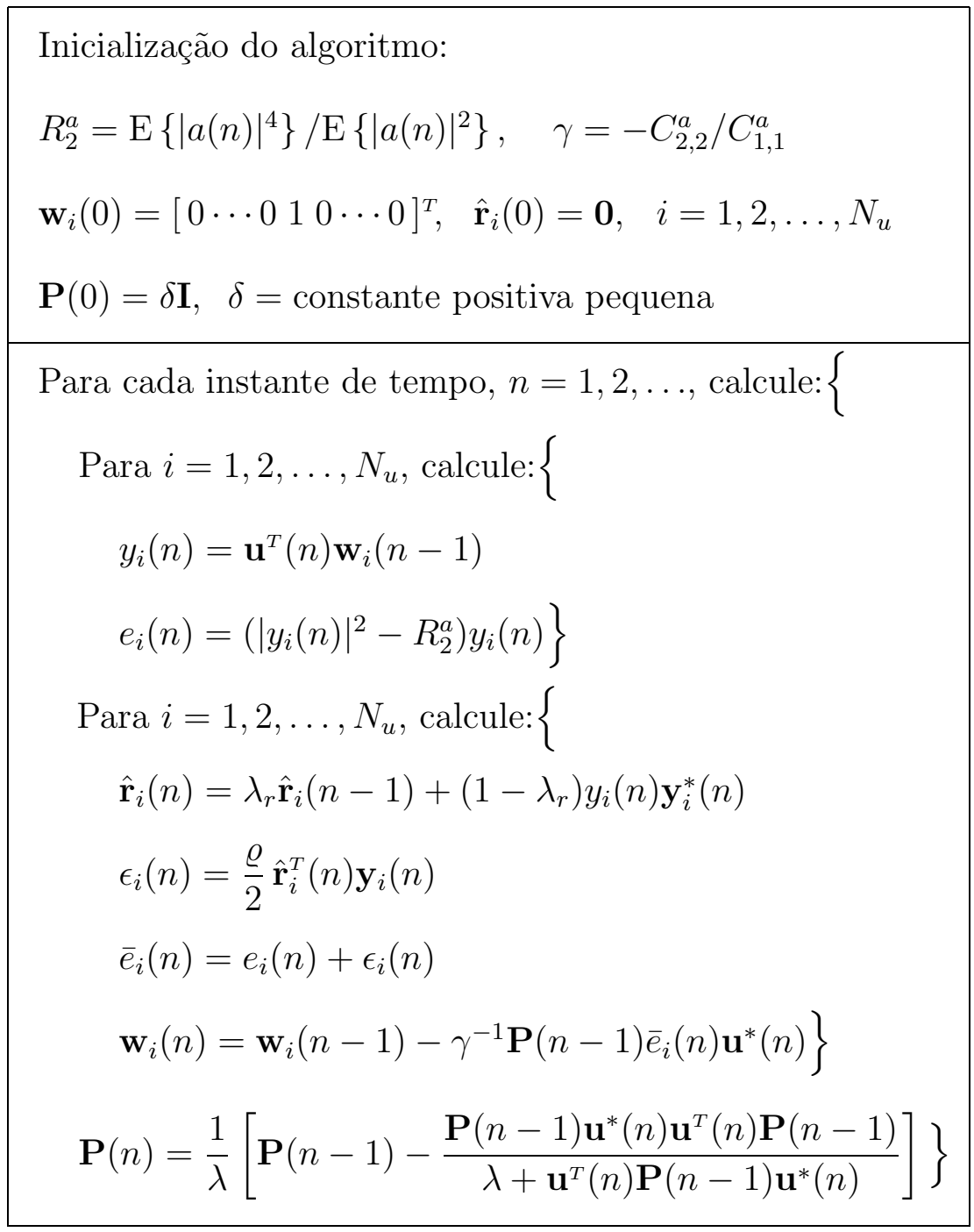

Tabela 4.2: Sumário do MU-SWA.

Considerando representação finita, a matriz de autocorrelação também pode deixar de ser positiva definida, causando problemas de instabilidade numérica no MU-SWA. Entretanto, os métodos que asseguram a positividade dessa matriz são bem conhecidos na literatura [Haykin, 1996]. Além disso, a fim de evitar instabilidade numérica e diminuir sua complexidade computacional, ele pode ser implementado respectivamente com decomposição QR e uma estrutura lattice [Martone, 1997; Gomes \& Barroso, 1998; Chi et al., 2000]. Isso faz com que o MU-SWA seja uma boa alternativa para equalização autodidata em sistemas MIMO.

Na Figura 4.1, são esquematizadas as relações entre os algoritmos citados. Cabe observar que todos eles podem ser derivados da função custo de Godard para o caso multiusuário e interpretados como algoritmos do gradiente estocástico. Desta forma, eles obedecem a Equação (4.13), sendo $\alpha(n)$ o passo de adaptação que, dependendo do algoritmo, pode ser um escalar ou uma matriz. 


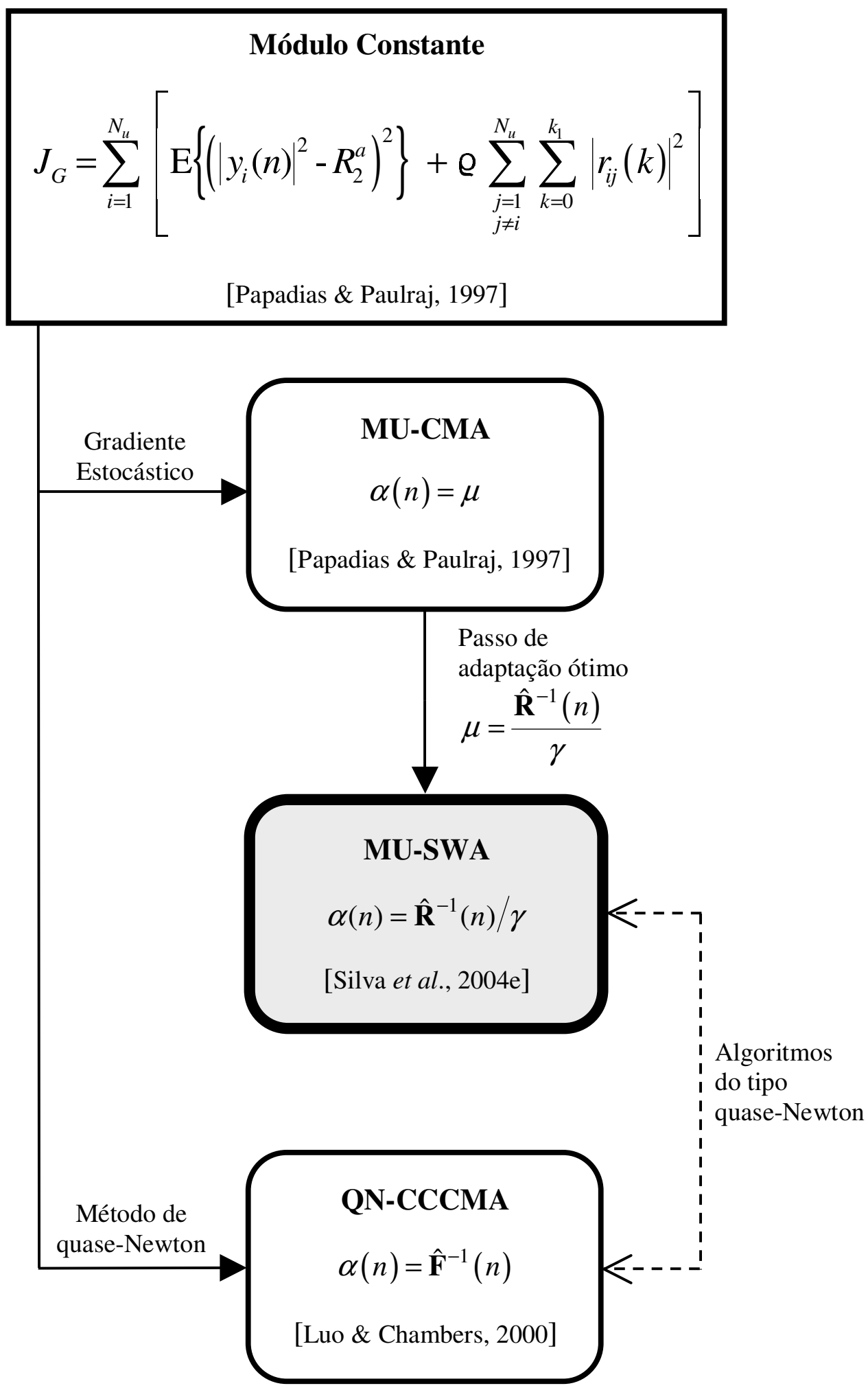

Figura 4.1: Relações entre algoritmos autodidatas para equalização espaço-temporal.

Comparando os algoritmos do Módulo Constante dos casos SISO e MIMO, constata-se que a diferença entre eles está basicamente no cálculo do erro, como pode ser observado nas tabelas 4.1 e 3.1 das páginas 110 e 73 respectivamente. Baseando-se nessa constatação, 
pode-se obter uma versão do algoritmo do Módulo Constante Acelerado para equalização espaço-temporal, substituindo-se o erro $e(n)$ da Tabela 3.3 (página 80) por $\bar{e}_{i}(n)$ definido em (4.6). As operações do algoritmo resultante são mostradas na Tabela 4.3. Como ele não foi obtido diretamente da minimização da função custo (4.2) e ainda utiliza a segunda derivada da estimativa dos coeficientes, fica difícil inseri-lo no esquema da Figura 4.1. Como no caso monousuário, resultados de simulações sugerem que escolhendo $m_{1} \approx 0,15$ e $\kappa \approx 100$, deve-se variar o parâmetro $\mu$ no intervalo ]0, 1[ para obter um desempenho adequado do algoritmo.

$$
\begin{aligned}
& \text { Inicialização do algoritmo: } \\
& R_{2}^{a}=\mathrm{E}\left\{|a(n)|^{4}\right\} / \mathrm{E}\left\{|a(n)|^{2}\right\}, \quad \mathbf{w}_{i}(0)=[0 \cdots 010 \cdots 0]^{T}, \\
& \mathbf{g}_{i}(0)=\mathbf{0}, \quad \hat{\mathbf{r}}_{i}(0)=\mathbf{0}, \quad i=1,2, \ldots, N_{u} \\
& \beta=2(=3) \text { para o caso complexo (real) } \\
& \text { Para cada instante de tempo, } n=1,2, \ldots, \text { calcule: }\{ \\
& \text { Para } i=1,2, \ldots, N_{u}, \text { calcule: }\{ \\
& y_{i}(n)=\mathbf{u}^{T}(n) \mathbf{w}_{i}(n-1) \\
& \left.e_{i}(n)=\left(\left|y_{i}(n)\right|^{2}-R_{2}^{a}\right) y_{i}(n)\right\} \\
& \operatorname{Para}_{i}=1,2, \ldots, N_{u}, \text { calcule: }\{ \\
& \hat{\mathbf{r}}_{i}(n)=\lambda_{r} \hat{\mathbf{r}}_{i}(n-1)+\left(1-\lambda_{r}\right) y_{i}(n) \mathbf{y}_{i}^{*}(n) \\
& \epsilon_{i}(n)=\frac{\varrho}{2} \hat{\mathbf{r}}_{i}^{T}(n) \mathbf{y}_{i}(n) \\
& \bar{e}_{i}(n)=e_{i}(n)+\epsilon_{i}(n) \\
& b_{i}(n)=\mu m_{1} \kappa^{2}+\mu^{2} m_{1} \kappa\left(\beta\left|y_{i}(n)\right|^{2}-R_{2}^{a}\right) \\
& c_{i}(n)=\frac{b_{i}(n) \mathbf{u}^{T}(n) \mathbf{g}_{i}(n-1)+\mu m_{1}(\mu+\kappa) \bar{e}_{i}(n)}{\mu+\kappa+b_{i}(n)\|\mathbf{u}(n)\|^{2}} \\
& \mathbf{w}_{i}\left(\mathbf{g}_{i}(n-1)-c_{i}(n) \mathbf{u}^{*}(n)\right]
\end{aligned}
$$

Tabela 4.3: Sumário do MU-AC-CMA. 
Na Tabela 4.4, são apresentadas as complexidades computacionais dos algoritmos. Cabe observar que como no caso monousuário, o MU-AC-CMA apresenta uma complexidade computacional que fica entre a do MU-SWA e a do MU-CMA.

\begin{tabular}{|c|c|c|c|c|}
\hline Op. & QN-CCCMA & MU-SWA & MU-AC-CMA & MU-CMA \\
\hline$\times$ & $6 M_{u}^{2}+M_{u}\left(N_{u}+D\right.$ & $4 M_{u}^{2}+M_{u}\left(2 N_{u}+\right.$ & $M_{u}\left(5 N_{u}+1\right)+$ & $M_{u}\left(2 N_{u}\right)+$ \\
& $+1)+2 D+3 N_{u}+2$ & $+1)+N_{u}(3 D+5)$ & $N_{u}(3 D+8)$ & $+N_{u}(3 D+5)$ \\
\hline$\div$ & 4 & 1 & $N_{u}$ & - \\
\hline SQRT & $M_{u}$ & - & - & - \\
\hline
\end{tabular}

Tabela 4.4: Complexidade computacional dos algoritmos autodidatas no caso MIMO para sinais reais, $\quad D=\left(N_{u}-1\right)\left(2 k_{1}+1\right), \quad N_{u} \geq 2$.

\subsection{Análise de tracking}

Todos os algoritmos da Seção 4.2 podem ser interpretados como algoritmos do tipo quaseNewton. Além disso, com exceção do MU-AC-CMA, eles podem ser descritos pela equação

$$
\mathbf{w}_{i}(n)=\mathbf{w}_{i}(n-1)-\rho(n) \widehat{\mathbf{F}}^{-1}(n) \mathbf{u}^{*}(n) \bar{e}_{i}(n),
$$

sendo $\widehat{\mathbf{F}}$ uma estimativa da matriz hessiana e $\bar{e}_{i}(n)$ o erro no caso MIMO, definido em (4.6). Embora o MU-AC-CMA possa ser interpretado como um algoritmo quase-Newton, ele não satisfaz uma equação do tipo (4.14), já que a estimativa do seu gradiente depende do incremento do vetor de coeficientes. Algoritmos da forma (4.14) satisfazem a relação de conservação de energia e podem ser descritos por um mapeamento sem perdas, como mostrado na Seção 3.4. É para essa classe de algoritmos que é feita a análise de tracking.

Assume-se a variação na solução ótima de zero-forcing para o usuário $i$ do tipo

$$
\mathbf{w}_{\mathrm{o}, i}(n)=\mathbf{w}_{\mathrm{o}, i}(n-1)+\mathbf{q}_{i}(n)
$$

sendo $\mathbf{q}_{i}(n)$ uma seqüência i.i.d com matriz de autocorrelação $\mathbf{Q}_{i}=\mathrm{E}\left\{\mathbf{q}_{i}^{*}(n) \mathbf{q}_{i}^{T}(n)\right\}$ positivadefinida. Além disso, considera-se que essa seqüência é independente das condições iniciais $\left\{\mathbf{w}_{\mathrm{o}, i}(-1), \mathbf{w}_{i}(-1)\right\}$ e de $\{\mathbf{u}(l)\}$ para todo $l<n$ [Sayed, 2003, Seção 7.4]. Definindo o erro 
dos coeficientes da forma $\widetilde{\mathbf{w}}_{i}(n)=\mathbf{w}_{\mathrm{o}, i}(n)-\mathbf{w}_{i}(n)$, a Equação (4.14) pode ser reescrita como

$$
\widetilde{\mathbf{w}}_{i}(n)-\mathbf{q}_{i}(n)=\widetilde{\mathbf{w}}_{i}(n-1)+\rho(n) \widehat{\mathbf{F}}^{-1}(n) \mathbf{u}^{*}(n) \bar{e}_{i}(n)
$$

Além do índice do usuário $i$, o que difere esta da Equação (3.40, página 85) é a definição do erro $\bar{e}_{i}(n)$. Como pode ser observado em (4.6), o erro no caso MIMO considera as correlações cruzadas entre os usuários. Desta forma, a análise de tracking dos algoritmos da forma (4.14) não é uma extensão direta da análise do caso SISO da Seção 3.5, já que considerações a respeito dos termos de correlações cruzadas devem ser levadas em conta.

Considera-se ausência de ruído e assume-se sem perda de generalidade que a saída do $i$-ésimo equalizador converge assintoticamente para a $i$-ésima fonte com atraso $\tau_{d, i}$. Além disso, todas as fontes são consideradas com mesmas características estatísticas, ou seja, $\mathrm{E}\left\{\left|a_{i}(n)\right|^{l}\right\}=\mathrm{E}\left\{|a(n)|^{l}\right\}$, para $l=1,2, \ldots$ e $i=1,2, \ldots, N_{u}$. O erro a priori e o MSE em regime para o $i$-ésimo equalizador são definidos respectivamente como

$$
e_{a, i}(n)=\mathbf{u}^{T}(n) \widetilde{\mathbf{w}}_{i}(n-1)
$$

e

$$
\zeta_{i}=\lim _{n \rightarrow \infty} \mathrm{E}\left\{\left|e_{a, i}(n)\right|^{2}\right\}
$$

A análise de tracking para os algoritmos da forma (4.14) é baseada nas seguintes suposições:

M1. $\mathrm{E}\{a(n)\}=0, \gamma>0$ e para sinais complexos $\mathrm{E}\left\{a^{2}(n)\right\}=0$ (simetria circular). Essa consideração é válida para a maioria das constelações utilizadas em comunicações digitais [Shalvi \& Weinstein, 1994; Sayed, 2003].

M2. Algoritmos da forma (4.14) obedecem a seguinte condição de regime $(n \rightarrow \infty)$ $\mathrm{E}\left\{\left\|\widetilde{\mathbf{w}}_{i}(n)\right\|_{\widehat{\mathbf{F}}}^{2}\right\}=\mathrm{E}\left\{\left\|\widetilde{\mathbf{w}}_{i}(n-1)\right\|_{\widehat{\mathbf{F}}}^{2}\right\}$ [Sayed, 2003, Seção 6.9.3].

M3. $a_{i}\left(n-\tau_{d}\right)$ e $e_{a, j}(n), j=1,2, \ldots, i, \ldots, N_{u}$ são independentes em regime. Essa consideração é uma generalização da assumida na análise de MSE e tracking do CMA [Yousef \& Sayed, 1999], na qual o caso $i=j$ foi especificado [Luo \& Chambers, 2002].

M4. $\|\mathbf{u}(n)\|_{\widehat{\mathbf{F}}^{-1}}^{2}$ e $\bar{e}_{i}(n)$ são independentes em regime. Essa consideração foi feita em [Luo \& Chambers, 2002] e é uma extensão da consideração S4 para o caso MIMO. 
M5. A Equação (4.4, página 108) pode ser reescrita utilizando-se a aproximação $y_{i}(n) \approx a_{i}\left(n-\tau_{d, i}\right)-e_{a, i}(n)$, já que em regime $a_{i}\left(n-\tau_{d, i}\right) \approx \mathbf{u}^{T}(n) \mathbf{w}_{\mathrm{o}, i}(n-1)$.

M6. Os erros de estimação nas saídas dos diferentes equalizadores são assumidos nãocorrelacionados, isto é, $\mathrm{E}\left\{e_{a, i}(n) e_{a, j}(n-k)\right\}=0$ para $i \neq j$. Segundo Luo e Chambers (2002), essa consideração é realista quando o equalizador for longo o suficiente para que sua saída convirja assintoticamente para uma estimativa da fonte correspondente.

M7. A quantidade $\hat{r}_{i j, k}(n-1)$, que é uma estimativa da correlação cruzada $\mathrm{E}\left\{y_{i}(n-1) y_{j}^{*}(n-\right.$ $1-k)\}$, é não-correlacionada com as saídas $y_{i}^{*}(n)$ e $y_{j}(n-k)$, quando $\hat{r}_{i j, k}(n-1)$ converge para uma constante em regime. Essa consideração é razoável quando se estima a correlação cruzada com uma janela exponencial, utilizando-se um fator de esquecimento $\lambda_{r}$ bem próximo de 1 [Luo \& Chambers, 2002].

M8. As matrizes $\hat{\mathbf{r}}_{i}^{*}(n) \hat{\mathbf{r}}_{i}^{T}(n)$ e $\mathbf{y}_{i}^{*}(n) \mathbf{y}_{i}^{T}(n)$ são não-correlacionadas em regime. Essa consideração é razoável, já que $\hat{\mathbf{r}}_{i}(n)$ tende para um vetor com elementos constantes em regime [Luo \& Chambers, 2002].

M9. $\lim _{n \rightarrow \infty} \mathrm{E}\left\{\hat{\mathbf{r}}_{i}^{T}(n) \mathbf{y}_{i}^{*}(n)\right\}=0$. A justificativa para essa consideração é similar às das considerações M7 e M8 [Luo \& Chambers, 2002].

M10. $\lim _{n \rightarrow \infty} \mathrm{E}\left\{e_{a, i}^{*}(n) \bar{e}_{i}(n)+e_{a, i}(n) \bar{e}_{i}^{*}(n)\right\} \approx-2 \gamma \zeta_{i}$. Essa consideração é obtida usando M3, M5, M9 e assumindo que $\gamma \mathrm{E}\left\{\left|e_{a, i}(n)\right|^{2}\right\} \gg \mathrm{E}\left\{\left|e_{a, i}(n)\right|^{4}\right\}$ [Mai \& Sayed, 2000, Th. 3 e Th. 4].

M11. $\lim _{n \rightarrow \infty} \mathrm{E}\left\{\left|\bar{e}_{i}(n)\right|^{2}\right\} \approx \mathrm{E}\left\{|a(n)|^{6}-\left(R_{2}^{a}\right)^{2}|a(n)|^{2}\right\}+\mathcal{K} \mathrm{E}\left\{|a(n)|^{2}\right\}^{3} \triangleq \bar{\xi}$, sendo

$$
\mathcal{K}=\frac{\varrho^{2}}{4}\left(k_{1}-1\right)\left(N_{u}-1\right) \frac{1-\lambda_{r}}{1+\lambda_{r}} .
$$

Nessa consideração, além do uso de M1, M3, M5, M7 e M8, os termos que dependem de $\mathrm{E}\left\{\left|e_{a, i}(n)\right|^{l}\right\}$ com $l=2,4,6$, foram assumidos pequenos em relação a $\mathrm{E}\left\{|a(n)|^{6}\right.$ $\left.\left(R_{2}^{a}\right)^{2}|a(n)|^{2}\right\}$ [Mai \& Sayed, 2000, Th. 3 e Th. 4]. Ela também pode ser obtida, assumindo-se a aproximação $\mathrm{E}\left\{\left|y_{i}(n)\right|^{l}\right\} \approx \mathrm{E}\left\{|a(n)|^{l}\right\} \quad \operatorname{com} \quad l=2,4,6$, na média quadrática de (4.4). 
As considerações M1-M5 são extensões diretas das S1-S5, feitas na Seção 3.5 para o caso SISO. As considerações M6-M9 aparecem devido ao termo que penaliza as correlações cruzadas entre os diferentes usuários e foram obtidas na análise de MSE do MU-CMA [Luo \& Chambers, 2002]. Pode-se então estabelecer o seguinte Teorema.

Teorema 4 Sob as considerações M1-M11, o erro médio quadrático em regime $(n \rightarrow \infty)$ dos algoritmos da forma (4.14) pode ser aproximado por

$$
\zeta_{i} \approx \frac{1}{2 \gamma \rho(n)}\left(\rho^{2}(n) \mathrm{E}\left\{\|\mathbf{u}(n)\|_{\widehat{\mathbf{F}}^{-1}}^{2}\right\} \bar{\xi}+\mathrm{E}\left\{\left\|\mathbf{q}_{i}(n)\right\|_{\widehat{\mathbf{F}}}^{2}\right\}\right)
$$

Demonstração: Calculando a norma ponderada ao quadrado de ambos os lados de (4.16), usando $\widehat{\mathbf{F}}(n)$ como matriz de ponderação, obtém-se

$$
\begin{gathered}
\left\|\widetilde{\mathbf{w}}_{i}(n)-\mathbf{q}_{i}(n)\right\|_{\widehat{\mathbf{F}}}^{2}=\left\|\widetilde{\mathbf{w}}_{i}(n-1)\right\|_{\widehat{\mathbf{F}}}^{2}+\rho(n)\left[\bar{e}_{i}(n) e_{a, i}^{*}(n)+\bar{e}_{i}^{*}(n) e_{a, i}(n)\right] \\
+\rho^{2}(n)\|\mathbf{u}(n)\|_{\widehat{\mathbf{F}}^{-1}}^{2}\left|\bar{e}_{i}(n)\right|^{2}
\end{gathered}
$$

Tomando a esperança de ambos os lados de (4.21) usando M2, chega-se a

$$
\begin{aligned}
-\mathrm{E}\left\{e_{a, i}^{*}(n) \bar{e}_{i}(n)+e_{a, i}(n) \bar{e}_{i}^{*}(n)\right\} \approx & \rho(n) \mathrm{E}\left\{\|\mathbf{u}(n)\|_{\widehat{\mathbf{F}}^{-1}}^{2}\left|\bar{e}_{i}(n)\right|^{2}\right\} \\
& +\rho^{-1}(n) \mathrm{E}\left\{\left\|\mathbf{q}_{i}(n)\right\|_{\widehat{\mathbf{F}}}^{2}\right\}
\end{aligned}
$$

Usando M4, M10 e M11 em (4.22), obtém-se (4.20), o que completa a demonstração.

Cabe observar que $\bar{\xi}$ definido na consideração M11 pode ser escrito como

$$
\bar{\xi}=\xi+\mathcal{K} E\left\{|a(n)|^{2}\right\}^{3}
$$

sendo

$$
\xi=\mathrm{E}\left\{|a(n)|^{6}-\left(R_{2}^{a}\right)^{2}|a(n)|^{2}\right\}
$$

o escalar que aparece na análise de tracking do caso SISO e $\mathcal{K}$ definido em (4.19). Considerando apenas um usuário, ou seja, fazendo $N_{u}=1$ em (4.19), obtêm-se $\mathcal{K}=0$ e $\bar{\xi}=\xi$. Neste caso, o Teorema 4 se reduz ao Teorema 3. Desta forma, conclui-se que o Teorema 3 pode ser interpretado como um caso particular do Teorema 4. 
As expressões do MSE em regime para o MU-CMA e o MU-SWA são obtidas do Teorema 4 e mostradas na Tabela 4.5. Comparando esta com a Tabela 3.5 do caso SISO, observase que a diferença está basicamente nos escalares $\bar{\xi}$ e $\xi$. Desta forma, as expressões de MSE em regime do caso MIMO podem ser obtidas a partir das do caso SISO e vice-versa. Conseqüentemente, a razão entre os valores de MSE mínimo do MU-SWA e do MU-CMA é equivalente à (3.45, página 87), obtida para o SWA e o CMA. Diante disso, embora a extensão da análise não tenha sido direta, as conclusões obtidas no caso SISO podem ser estendidas para o caso MIMO, ou seja, dependendo da forma da matriz $\mathbf{Q}_{i}$, a capacidade de tracking do MU-CMA é melhor do que a do MU-SWA e vice-versa. Esses algoritmos apresentam capacidades de tracking iguais quando $\mathbf{Q}_{i}=\sigma_{q_{i}}^{2} \mathbf{I}$. Um outro resultado que também pode ser usado no caso MIMO é o da Equação (3.47, página 88). Através dessa equação, os parâmetros do MU-CMA e MU-SWA podem ser ajustados para que eles atinjam o mesmo MSE em regime no caso estacionário.

\begin{tabular}{|c|c|c|}
\hline Algoritmo & MU-CMA & MU-SWA \\
\hline$\rho(n)$ & $\mu$ & $\gamma^{-1}$ \\
\hline$\widehat{\mathbf{F}}(n)$ & $\mathbf{I}$ & $\sum_{l=1}^{n} \lambda^{n-l} \mathbf{u}^{*}(l) \mathbf{u}(l)$ \\
\hline $\mathrm{E}\left\{\|\mathbf{u}(n)\|_{\hat{\mathbf{F}}^{-1}}^{2}\right\}$ & $\operatorname{Tr}(\mathbf{R})$ & $(1-\lambda) M$ \\
\hline $\mathrm{E}\left\{\left\|\mathbf{q}_{i}(n)\right\|_{\widehat{\mathbf{F}}}^{2}\right\}$ & $\operatorname{Tr}\left(\mathbf{Q}_{i}\right)$ & $\frac{\operatorname{Tr}\left(\mathbf{Q}_{i} \mathbf{R}\right)}{1-\lambda}$ \\
\hline$\zeta_{i}$ & $\frac{\mu \bar{\xi} \operatorname{Tr}(\mathbf{R})}{2 \gamma}+\frac{\operatorname{Tr}\left(\mathbf{Q}_{i}\right)}{2 \gamma \mu}$ & $\frac{M(1-\lambda) \bar{\xi}}{2 \gamma^{2}}+\frac{\operatorname{Tr}\left(\mathbf{Q}_{i} \mathbf{R}\right)}{2(1-\lambda)}$ \\
\hline$\nu_{\mathrm{o}}$ & $\mu_{o}=\sqrt{\frac{\operatorname{Tr}\left(\mathbf{Q}_{i}\right)}{\bar{\xi} \operatorname{Tr}(\mathbf{R})}}$ & $1-\lambda_{o}=\sqrt{\frac{\gamma^{2} \operatorname{Tr}\left(\mathbf{Q}_{i} \mathbf{R}\right)}{M \bar{\xi}}}$ \\
\hline$\zeta_{o}$ & $\sqrt{\gamma^{-2} \bar{\xi} \operatorname{Tr}(\mathbf{R}) \operatorname{Tr}\left(\mathbf{Q}_{i}\right)}$ & $\sqrt{\gamma^{-2} M \bar{\xi} \operatorname{Tr}\left(\mathbf{Q}_{i} \mathbf{R}\right)}$ \\
\hline
\end{tabular}

Tabela 4.5: MSE $\left(\zeta_{i}\right)$ em regime, parâmetros de adaptação ótimos $\left(\nu_{\mathrm{o}}\right)$ e valores mínimos do MSE em regime $\left(\zeta_{o}\right)$ para o MU-CMA e o MU-SWA; $\mathbf{R}$ é a matriz de autocorrelação. 
É importante ressaltar que essa análise é válida para constelações de módulo não-constante. Para constelações de módulo constante, a maior parte do MSE é devido ao termo das correlações cruzadas. Neste caso, como observado em [Luo \& Chambers, 2002], há uma diferença relativamente grande entre os valores de MSE obtidos através de simulação e os obtidos a partir da análise. Uma análise de tracking para algoritmos autodidatas da forma (4.14), considerando constelações de módulo constante, é um problema em aberto na literatura.

\subsection{Resultados experimentais}

Nesta seção, apresentam-se os resultados de simulações com os algoritmos autodidatas do gradiente estocástico no caso MIMO. Como medida de desempenho utiliza-se a interferência residual (IR) definida como

$$
\operatorname{IR}\left(\mathbf{s}_{i}\right)=\frac{\sum_{k}\left|s_{k}\right|^{2}}{\left|s_{k_{m}}\right|^{2}}-1
$$

sendo $\mathbf{s}_{i}$ o vetor de resposta combinada do usuário $i$ e $s_{k_{m}}$ seu componente de maior valor absoluto [Shalvi \& Weinstein, 1994; Lambert \& Nikias, 2000]. Nas simulações, os algoritmos foram ajustados para atingir o mesmo erro quadrático médio (MSE) em regime. Como no caso SISO, o passo de adaptação do MU-CMA foi escolhido em função do fator de esquecimento do MU-SWA, considerando-se a Equação (3.47, página 88) e os parâmetros do MU-AC-CMA foram ajustados experimentalmente.

Adota-se a seguinte notação para designar a inicialização dos filtros do equalizador

$$
\text { Inicialização: }\left(i_{1} ; i_{2} ; \cdots ; i_{k} ; \cdots ; i_{N_{u}}\right)_{M_{u}} \text {. }
$$

Isso significa que o Equalizador $k$ é inicializado com um vetor de dimensão $M_{u}$ contendo um único elemento não nulo na posição $i_{k}$. Para exemplificar, considera-se que $N_{u}=3, M_{u}=6$ e que os filtros do equalizador sejam inicializados com os vetores

$$
\mathbf{w}_{1}(0)=\left[\begin{array}{llllll}
1 & 0 & 0 & 0 & 0 & 0
\end{array}\right]^{T}, \quad \mathbf{w}_{2}(0)=\left[\begin{array}{llllll}
0 & 0 & 1 & 0 & 0 & 0
\end{array}\right]^{T} \quad \mathbf{w}_{3}(0)=\left[\begin{array}{llllll}
0 & 0 & 0 & 0 & 1 & 0
\end{array}\right]^{T} .
$$

Neste caso, segundo a notação adotada, a inicialização deve ser designada como $(1 ; 3 ; 5)_{6}$.

Os coeficientes dos canais utilizados estão mostrados na Tabela 4.6. Além desses canais, considerou-se também um sistema MIMO com $N_{u}=2$ usuários, $K=3$ sensores e canais 
variantes no tempo $H_{i j}(z, n)=h_{0}^{i j}(n)+h_{1}^{i j}(n) z^{-1}+h_{2}^{i j}(n) z^{-2}$, cujos coeficientes são gerados a partir da filtragem do ruído branco gaussiano por um filtro Butterworth de segunda ordem, projetado para simular uma taxa de desvanecimento de 0,1 Hz [Shimamura \& Cowan, 1997; Cowan \& Semnani, 1998]. O conjunto dos canais $H_{i j}(z, n)$ para $i=1,2$ e $j=1,2,3$ é denotado como canal $\mathrm{H}_{13}$.

\begin{tabular}{|c|c|c|c||c|c|}
\hline \multicolumn{4}{|c||}{$H_{i j}$ do canal $\mathrm{H}_{12}$} & \multicolumn{2}{c|}{$H_{i j}$ do canal $\mathrm{H}_{14}$} \\
\hline$i j$ & $h_{0}$ & $h_{1}$ & $h_{2}$ & $h_{0}$ & $h_{1}$ \\
\hline 11 & $+0,43+j 0,36$ & $+0,46+j 0,53$ & $-0,30-j 0,33$ & $-0,6$ & $+1,2$ \\
21 & $+0,21+j 0,15$ & $-0,26-j 0,91$ & $+0,16-j 0,08$ & $+0,1$ & $-0,2$ \\
12 & $-0,37+j 0,20$ & $-0,87-j 0,12$ & $+0,15-j 0,20$ & $+0,5$ & $-1,0$ \\
22 & $+0,55-j 0,25$ & $-0,61+j 0,06$ & $-0,09-j 0,50$ & $-0,6$ & $+0,9$ \\
13 & $+0,61+j 0,66$ & $+0,12-j 0,31$ & $-0,24-j 0,17$ & $+0,4$ & $-0,2$ \\
23 & $+0,43+j 0,29$ & $-0,52-j 0,58$ & $+0,31+j 0,15$ & $-0,1$ & $+0,4$ \\
\hline
\end{tabular}

Tabela 4.6: Coeficientes dos canais $\mathrm{H}_{12}$ e $\mathrm{H}_{14}$.

O MU-CMA, o MU-AC-CMA e o MU-SWA são comparados durante a convergência através de curvas de interferência residual (IR) para cada um dos usuários, como mostrado na Figura 4.2. Essas curvas correspondem a uma média de 100 realizações, considerando 4-QAM e o canal $\mathrm{H}_{12}$ com $\mathrm{SNR}=30 \mathrm{~dB}$. Os algoritmos foram inicializados com $(5 ; 7)_{15}$ e seus fatores de adaptação ajustados para que atingissem o mesmo MSE em regime. Pode-se observar que, para os dois usuários, o MU-AC-CMA apresenta um comportamento próximo ao do MU-SWA e ambos possuem uma velocidade de convergência bem maior que a do MU-CMA. No caso do Equalizador 1, o MU-CMA converge para um mínimo local, enquanto os demais convergem para um patamar de IR próximo de -30 dB após 15000 iterações. Na Figura 4.3, são mostradas as constelações de saída dos equalizadores para uma realização, considerando as $10^{3}$ últimas iterações dos algoritmos. Passando os sinais de saída por decisores, verifica-se que, a menos de uma rotação de fase, os sinais transmitidos são recuperados. Para a saída do Equalizador 1, foi observado ainda que com uma relação sinal-ruído menor, o MU-CMA apresenta mais erros que os demais algoritmos ao tentar recuperar o sinal transmitido por um dos usuários. 

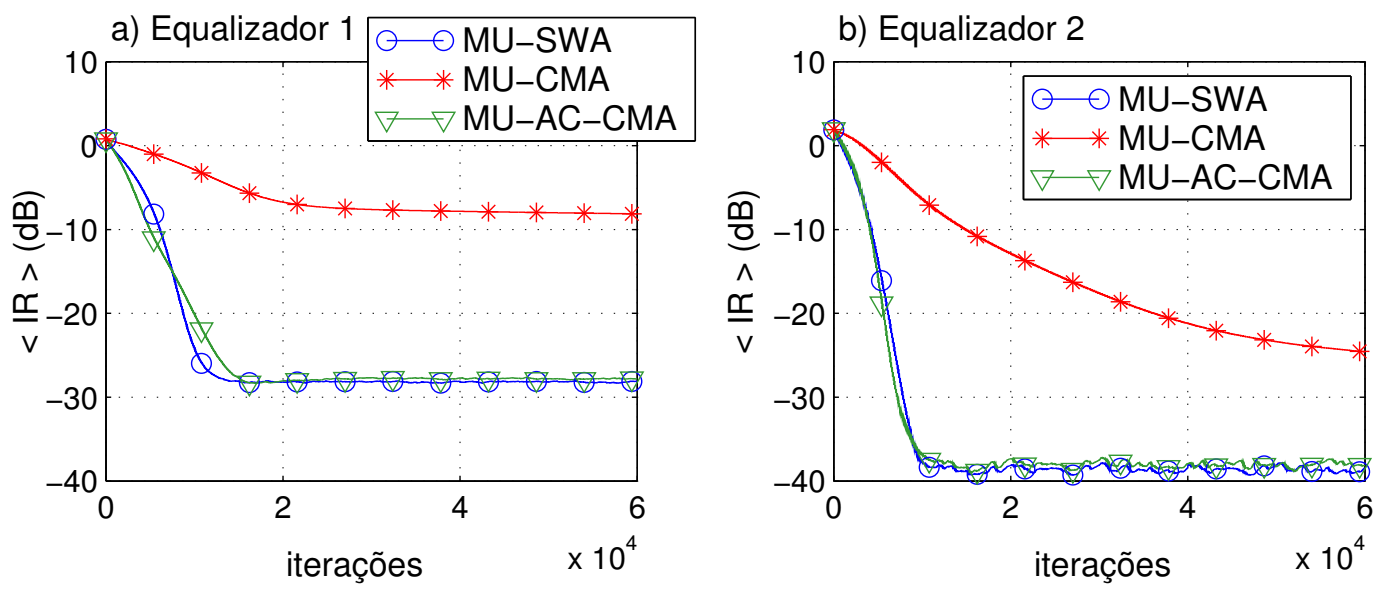

Figura 4.2: IR média (100 realizações) para o MU-SWA $\left(\lambda=0,9992, \delta=8 \times 10^{-4}\right)$, MU-CMA $\left(\mu=5 \times 10^{-5}, \varrho=3\right)$ e MU-AC-CMA $\left(\mu=0,05, m_{1}=0,15, \kappa=100, \varrho=3\right) ; N_{u}=2, K=3$, $M_{u}=15$, inicialização: $(5 ; 7)_{15}$, 4-QAM, canal $\mathrm{H}_{12}$ e $\mathrm{SNR}=30 \mathrm{~dB}$.
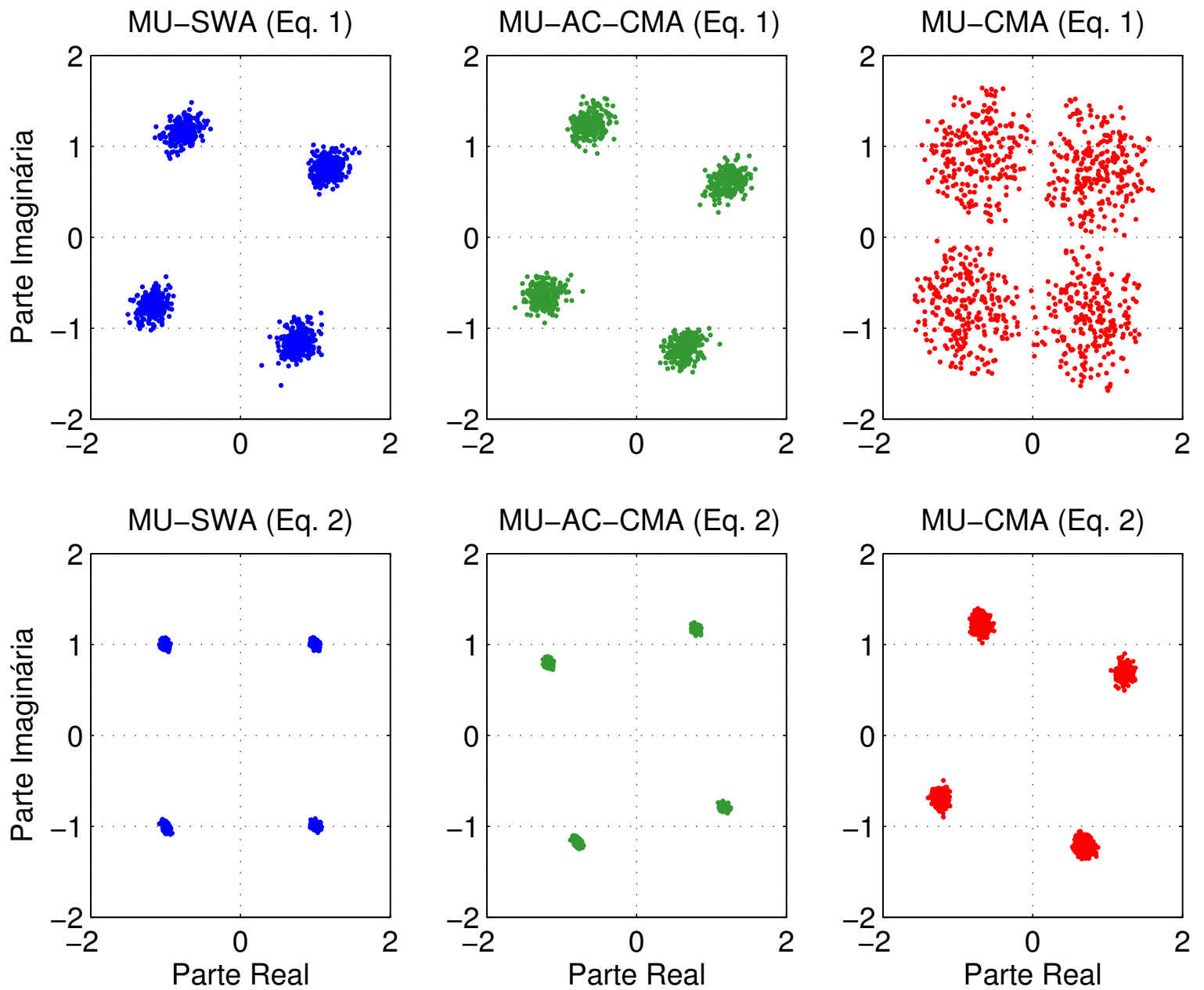

Figura 4.3: Sinais de saída dos equalizadores (Eq. 1 e Eq. 2) entre as iterações 59000 e 60000 para os algoritmos configurados como na simulação da Figura 4.2. 
Nas figuras 4.4 e 4.5, são verificadas as influências do passo de adaptação e da inicialização na convergência do MU-CMA e do MU-AC-CMA para a adaptação dos coeficientes do Equalizador 1. Inicializando os equalizadores com $(5 ; 7)_{15}$, considera-se a variação do passo de adaptação da forma $\mu=k \times 10^{-5}$, sendo $k$ uma constante positiva, conforme mostrado na Figura 4.4. Observa-se que o MU-CMA não converge efetivamente até $6 \times 10^{4}$ iterações, independente do passo de adaptação considerado. Desta forma, para efeitos práticos, verificase que ele atinge o mesmo nível de IR que o MU-AC-CMA apenas para $k=50$, mas com uma convergência mais lenta e uma variação maior. Para $k=100$, ele diverge, o que sugere que o intervalo do passo de adaptação que garante a convergência do MU-CMA é menor que o do MU-AC-CMA e que o passo "ótimo" desses algoritmos diferem. Isso justifica a diferença entre os valores de $k$ considerados para os dois algoritmos. O MU-AC-CMA é mais robusto que o MU-CMA, convergindo com uma velocidade maior para o mesmo patamar de IR, independente do passo de adaptação.
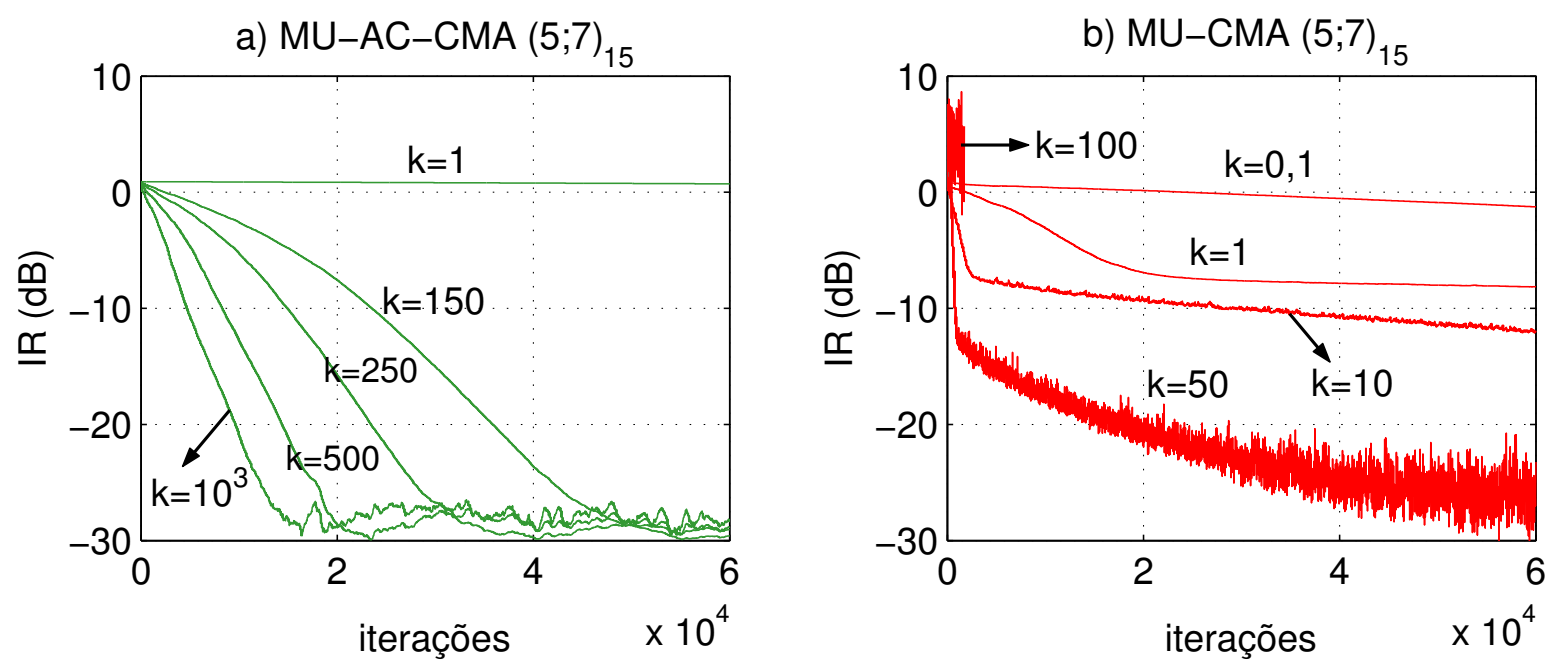

Figura 4.4: IR do a) MU-AC-CMA $\left(\mu=k \times 10^{-5}, \varrho=3\right)$ e MU-CMA $\left(\mu=k \times 10^{-5}, \kappa=100\right.$, $m_{1}=0,15, \varrho=3$ ), canal $\mathrm{H}_{12}, \mathrm{SNR}=30 \mathrm{~dB}, 4$-QAM, $N_{u}=2, K=3, M_{u}=15$, inicialização: $(5 ; 7)_{15}$, Equalizador 1.

Inicializando os equalizadores com $(8 ; 14)_{15}$, considera-se a variação do passo de adaptação mostrada na Figura 4.5. O comportamento dos algoritmos é similar ao do caso anterior. Cabe observar que o patamar de IR atingido pelo MU-AC-CMA é menor, mostrando que, como no caso SISO, a inicialização tem uma forte influência no desempenho dos algoritmos. No 
entanto, em ambos os casos o patamar de IR atingido pelo MU-AC-CMA é suficiente para recuperar o sinal transmitido. Esse comportamento sugere que, dada uma condição inicial, o MU-AC-CMA converge para o mesmo patamar de IR, independente do passo de adaptação e com uma velocidade de convergência maior que a do MU-CMA.
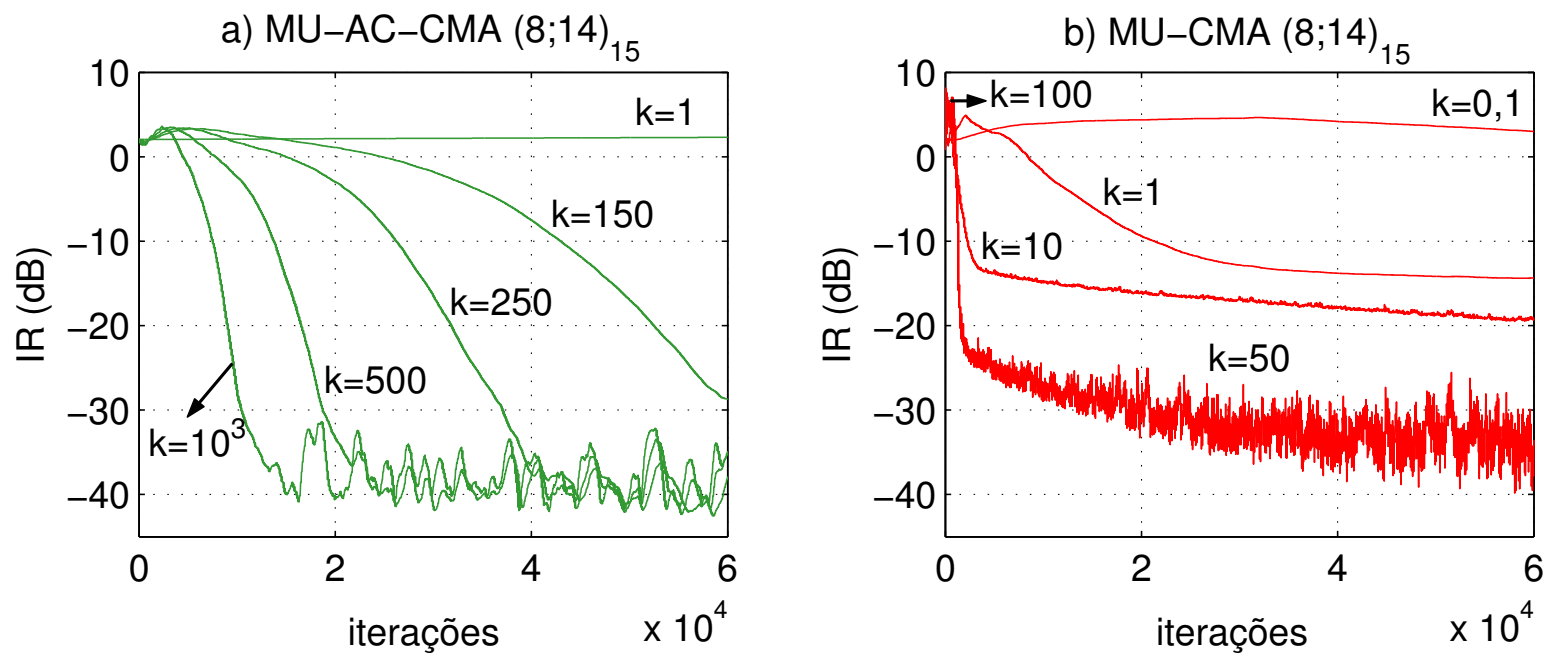

Figura 4.5: IR do a) MU-AC-CMA $\left(\mu=k \times 10^{-5}, \varrho=3\right)$ e MU-CMA $\left(\mu=k \times 10^{-5}, \kappa=100\right.$, $m_{1}=0,15, \varrho=3$ ), canal $\mathrm{H}_{12}, \mathrm{SNR}=30 \mathrm{~dB}, 4$-QAM, $N_{u}=2, K=3, M_{u}=15$, inicialização: $(8 ; 14)_{15}$, Equalizador 1.

Considerando um sistema MIMO com $N_{u}=2, K=3$ e $M_{u}=15$ e o canal $\mathrm{H}_{13}$ com canais variantes no tempo e $\mathrm{SNR}=25 \mathrm{~dB}$, obtêm-se os resultados mostrados nas figuras 4.6 e 4.7 . Na Figura 4.6, são mostrados os erros na recuperação do sinal binário $a_{1}(n)$ para o MU-CMA, MU-SWA e MU-AC-CMA. Como no caso SISO, os erros em seqüência podem ser associados a nulos espectrais do canal. O MU-SWA apresenta a convergência mais rápida seguido do MUAC-CMA e do MU-CMA. Neste caso, o MU-AC-CMA possui um desempenho próximo ao do MU-CMA, como pode ser observado na primeira linha da Tabela 4.7. Em contrapartida, na recuperação do sinal $a_{2}(n)$, o MU-AC-CMA passa a ter um comportamento próximo do MU-SWA, como pode ser observado na Figura 4.7 e na segunda linha da Tabela 4.7. Em cada caso, as curvas de interferência residual (IR) correspondentes são apresentadas nas figuras 4.6-d e 4.7-d. Cabe observar que o limiar de acerto de IR é em torno de $-8 \mathrm{~dB}$, ou seja, abaixo desse limiar os algoritmos conseguem recuperar os sinais transmitidos. Nessa simulação, o MU-AC-CMA apresenta um comportamento próximo ao do MU-CMA para 
o Equalizador 1 e próximo ao do MU-SWA para o Equalizador 2. Esse comportamento também foi observado no caso SISO e sugere que ele apresenta um desempenho intermediário, podendo funcionar ora como o MU-CMA, ora como o MU-SWA. O MU-SWA apresentou neste caso a melhor capacidade de tracking. No entanto, a matriz de autocorrelação pode deixar de ser positiva definida, o que pode causar problemas de instabilidade numérica. Neste caso, técnicas mais robustas, como decomposição QR, devem ser consideradas para evitar esse problema [Martone, 1997].

\begin{tabular}{|c|c|c|c|}
\hline Algoritmo & MU-SWA & MU-AC-CMA & MU-CMA \\
\hline $\begin{array}{c}\text { № de erros na } \\
\text { recup. de } a_{1}(n)\end{array}$ & 356 & 1461 & 1885 \\
\hline $\begin{array}{l}\text { № de erros na } \\
\text { recup. de } a_{2}(n)\end{array}$ & 103 & 262 & 1452 \\
\hline
\end{tabular}

Tabela 4.7: Número de erros na recuperação dos sinais $a_{1}(n)$ e $a_{2}(n)$ considerando $4 \times 10^{4}$ iterações dos algoritmos MU-SWA, MU-SWA e MU-AC-CMA.

Para verificar a validade da análise de tracking do MU-CMA e MU-SWA apresentada na Seção 4.3, assumem-se $\mathbf{Q}=\sigma_{q}^{2} \mathbf{I}$ e o canal $\mathrm{H}_{14}$, cujos coeficientes são mostrados na Tabela 4.6. Além disso, considera-se dois filtros no equalizador com $M_{u}=9$ coeficientes, $\sigma_{q}=10^{-3}$ e sinalização 4-PAM com estatísticas $E\left\{a^{6}(n)\right\}=365, \mathrm{E}\left\{a^{2}(n)\right\}=5$ e $R_{2}^{a}=8,2$. Na Figura 4.8, é mostrado o MSE em função dos parâmetros de adaptação, considerando resultados teóricos e experimentais para o MU-CMA e o MU-SWA. O valor do MSE experimental foi obtido como média de 100 realizações. Os resultados da simulação do MU-SWA apresentam uma boa concordância com os da análise em toda a faixa de $(1-\lambda)$ considerada. A concordância entre os resultados da simulação e análise do MU-CMA ocorre para $\mu<10^{-4}$. A partir desse valor, o algoritmo começa a divergir o que faz com que o resultado previsto pela análise não seja mais válido. 

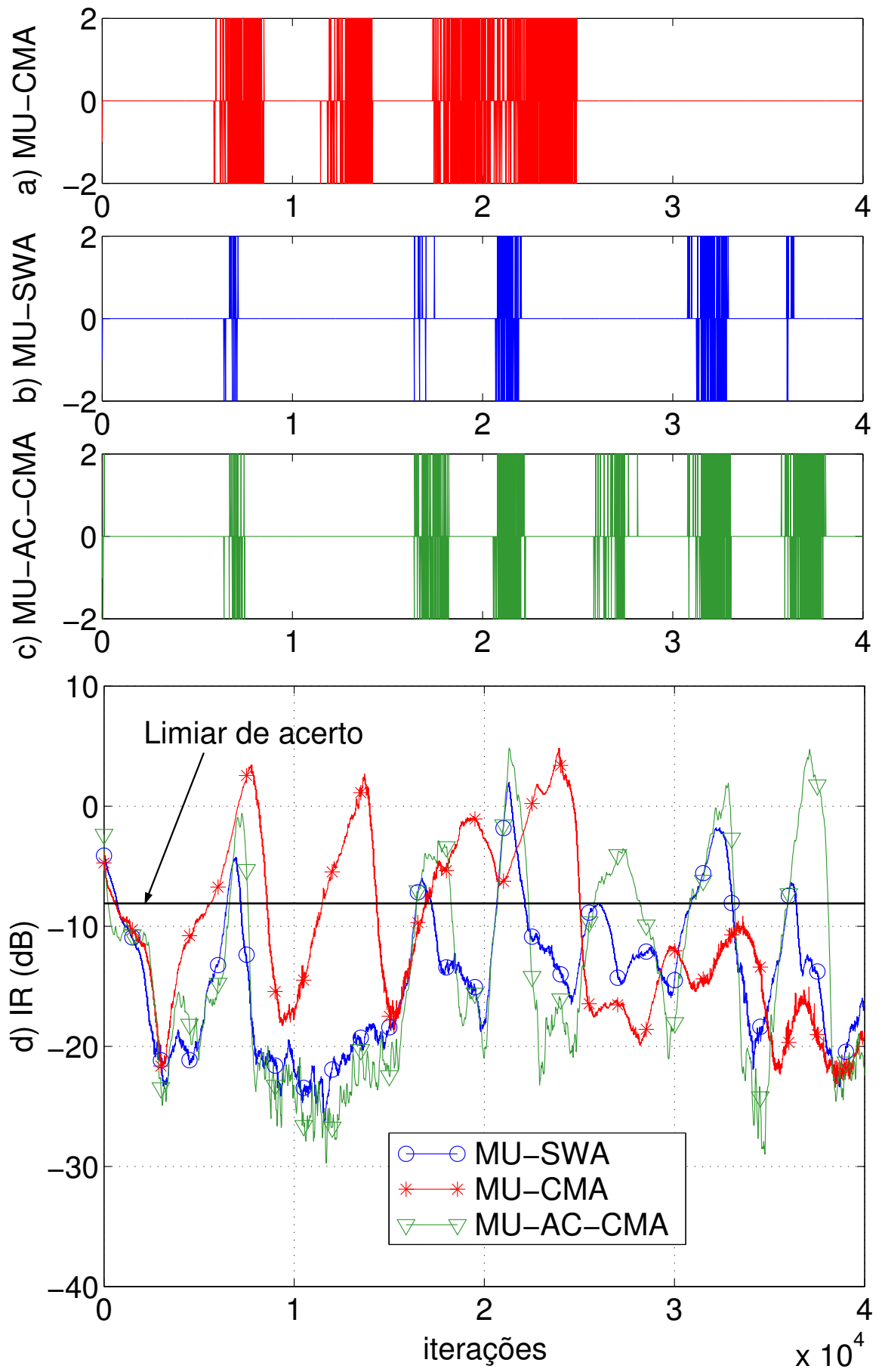

Figura 4.6: a), b) e c) Erros na recuperação do sinal $a_{1}(n)$ para o MU-CMA $(\mu=0,01, \varrho=4)$, o MU-SWA $(\lambda=0,995, \delta=0,005)$ e o MU-AC-CMA $\left(\mu=0,99, m_{1}=0,15, \kappa=100, \varrho=4\right)$ respectivamente; d) Interferência residual; $\mathrm{SNR}=25 \mathrm{~dB}, 2$-PAM, canal $\mathrm{H}_{13}, M_{u}=15, K=3 \mathrm{e}$ inicialização: $(5 ; 7) 15$. 

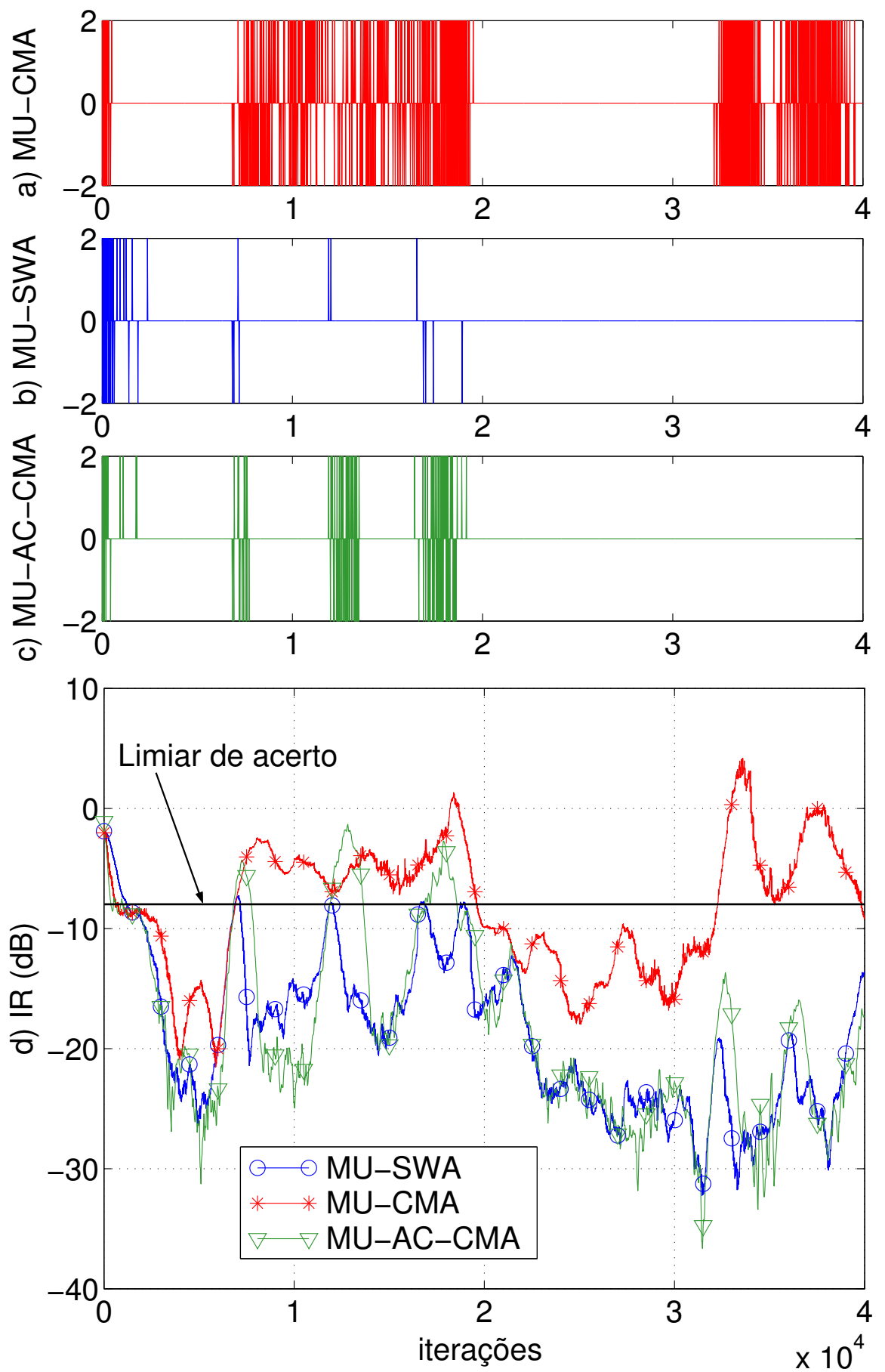

Figura 4.7: a), b) e c) Erros na recuperação do sinal $a_{2}(n)$ para o MU-CMA $(\mu=0,01, \varrho=4)$, o MU-SWA $(\lambda=0,995, \delta=0,005)$ e o MU-AC-CMA $\left(\mu=0,99, m_{1}=0,15, \kappa=100, \varrho=4\right)$ respectivamente; d) Interferência residual; $\mathrm{SNR}=25 \mathrm{~dB}, 2$-PAM, canal $\mathrm{H}_{13}, M_{u}=15, \mathrm{~K}=3 \mathrm{e}$ inicialização: $(5 ; 7) 15$. 


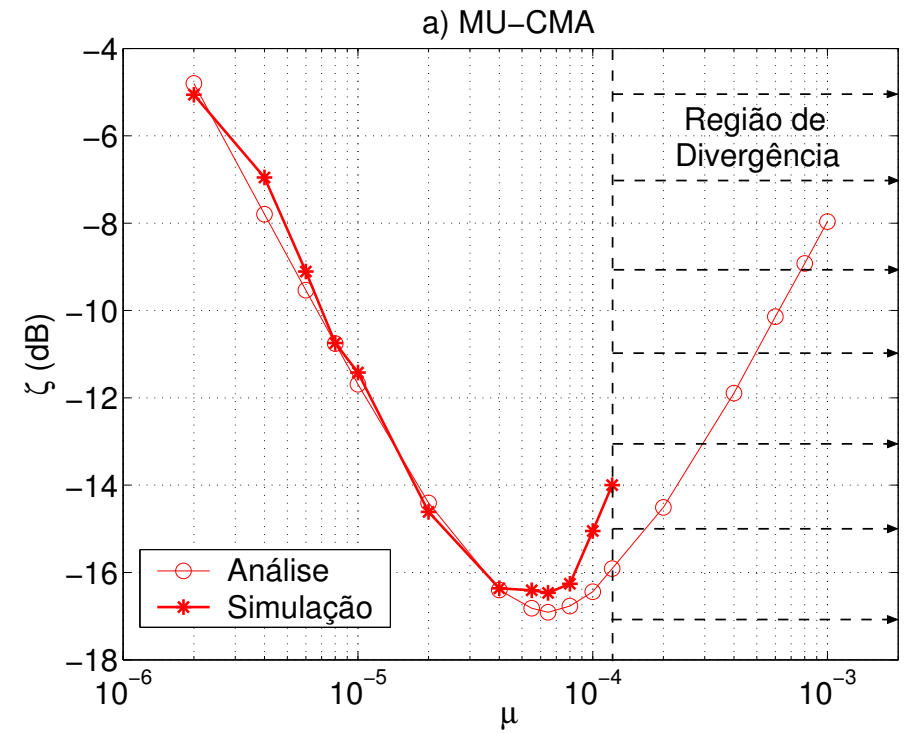

b) MU-SWA

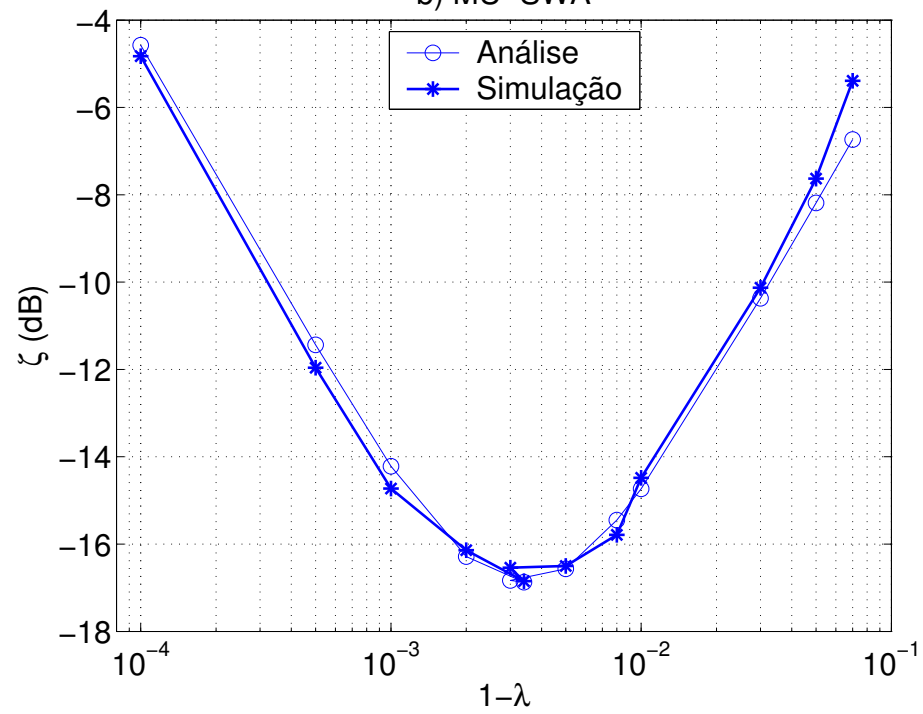

Figura 4.8: Curvas com resultados analíticos e de simulação do MSE; canal $\mathrm{H}_{14}, \mathbf{Q}=10^{-6} \mathbf{I}$, 4-PAM, $N_{u}=2, K=3, M_{u}=9$, inicialização: $(2 ; 5)_{9}$, média de 100 realizações, Equalizador 1; a) MU-CMA; e b) MU-SWA.

\subsection{Conclusões}

Neste capítulo, os algoritmos aproximados para equalização autodidata do caso SISO foram estendidos para o caso MIMO. Inspirando-se nas relações entre o CMA e o SWA, foi proposto um algoritmo denominado MU-SWA, que pode ser interpretado como uma extensão do SWA para o caso multiusuário. Às custas de uma complexidade computacional proporcional a $M_{u}^{2}$, ele apresenta uma velocidade de convergência maior que a do MU-CMA. No entanto, a matriz de autocorrelação pode perder sua positividade, causando problemas de 
instabilidade numérica, o que pode ser evitado, utilizando-se a decomposição QR. O algoritmo do tipo "acelerador", AC-CMA, também foi estendido para o caso MIMO, gerando um novo algoritmo para equalização espaço-temporal, denominado MU-AC-CMA. Como no caso SISO, ele requer um número de operações proporcional a $M_{u}$ e apresenta um desempenho intermediário com relação à velocidade de convergência e capacidade de tracking, tendo um comportamento ora próximo do MU-SWA, ora próximo do MU-CMA. Resultados de simulações sugerem que, dada uma condição inicial, o MU-AC-CMA converge para um mesmo patamar de interferência residual, independente do passo de adaptação e com uma velocidade de convergência maior que a do MU-CMA. Além disso, o intervalo do passo de adaptação que garante a convergência desse algoritmo parece ser maior que o do MU-CMA.

A análise de tracking feita no caso SISO também foi estendida para o caso MIMO. Neste caso, chegou-se ao Teorema 4, a partir das considerações M1-M11. Dentro da região de convergência dos algoritmos, houve uma boa concordância entre o MSE teórico previsto pelo Teorema 4 e o experimental. Cabe observar que o Teorema 3, obtido no caso SISO, é um caso particular do Teorema 4 , ou seja, fazendo $N_{u}=1$ no segundo, chega-se ao primeiro. Além disso, embora a extensão da análise do caso SISO não tenha sido direta, as conclusões obtidas puderam ser estendidas, mostrando que há situações em que o MU-CMA apresenta uma capacidade de tracking melhor que a do MU-SWA e vice-versa. É importante ressaltar que essa análise é válida dentro da região de convergência dos algoritmos e para constelações de módulo não-constante. Uma análise de tracking para constelações de módulo constante e a determinação da região de convergência dos algoritmos ainda são problemas em aberto.

A sobre-amostragem e os algoritmos concorrentes, abordados no Capítulo 3, também podem ser considerados no caso multiusuário. Essa extensão será deixada para um trabalho futuro. 


\section{Capítulo 5}

\section{Sobre equalizadores com}

\section{realimentação de decisões}

Neste capítulo, aborda-se o equalizador com realimentação de decisões (DFE - Decision Feedback equalizer) adaptado de forma autodidata. Inicialmente, são feitas considerações sobre o comportamento ideal do filtro de realimentação. O algoritmo que evita soluções degeneradas é revisitado e um algoritmo concorrente, denominado NDEG-CMA-SDD (NonDegenerative Soft Decision Directed Constant Modulus Algorithm), é proposto. Em seguida, a fim de evitar propagação de erros no DFE, considera-se um equalizador híbrido que utiliza uma rede neural recorrente no pré-processamento das decisões passadas. O capítulo termina com resultados de simulações e conclusões.

\subsection{O comportamento ideal do filtro de realimentação}

Utilizando as definições da Seção 1.3.2 e o esquema apresentado na Figura 1.9 da página 17, o sinal de saída do filtro direto do DFE pode ser escrito em função dos elementos do vetor de resposta combinada da cascata canal-filtro direto $\mathbf{s}_{f}(n-1)$, ou seja,

$$
y_{f}(n)=\sum_{k=0}^{M_{s}-1} s_{f, k}(n-1) a(n-k)+\underbrace{\sum_{k=0}^{M_{f}-1} w_{f, k}(n-1) \eta(n-k)}_{v(n)},
$$

sendo $M_{s}=N_{h}+M_{f}-1$ e $v(n)$ o resultado da convolução do ruído branco gaussiano com os coeficientes do filtro direto. Por conveniência reescreve-se (5.1) da seguinte forma 
[Szczecinski \& Gei, 2002]

$$
\begin{aligned}
y_{f}(n)= & s_{f, \tau_{d}}(n-1) a\left(n-\tau_{d}\right)+\sum_{k=0}^{\tau_{d}-1} s_{f, k}(n-1) a(n-k) \\
& +\sum_{k=1}^{M_{s}-\tau_{d}-1} s_{f, k+\tau_{d}}(n-1) a\left(n-\tau_{d}-k\right)+v(n) .
\end{aligned}
$$

Assumindo decisões corretas, ou seja,

$$
\hat{a}\left(n-\tau_{d}\right)=a\left(n-\tau_{d}\right)
$$

a saída do filtro de realimentação pode ser expressa como

$$
y_{b}(n)=\sum_{k=1}^{M_{b}} w_{b, k}(n-1) a\left(n-\tau_{d}-k\right) .
$$

Utilizando (5.2) e (5.4), são analisadas três situações distintas:

1. Supondo que $M_{b}=M_{s}-\tau_{d}-1$, a saída do DFE pode ser reescrita como

$$
\begin{aligned}
y(n)= & s_{f, \tau_{d}}(n-1) a\left(n-\tau_{d}\right)+\sum_{k=0}^{\tau_{d}-1} s_{f, k}(n-1) a(n-k) \\
& +\sum_{k=1}^{M_{s}-\tau_{d}-1}\left[s_{f, k+\tau_{d}}(n-1)+w_{b, k}(n-1)\right] a\left(n-\tau_{d}-k\right)+v(n) .
\end{aligned}
$$

É possível observar nessa expressão que, para eliminar completamente a ISI, os dois somatórios devem tender a zero. O filtro de realimentação do DFE vai agir somente sobre o segundo somatório e pode anulá-lo se

$$
w_{b, k}(n-1)=-s_{f, k+\tau_{d}}(n-1), \quad k=1, \ldots, M_{s}-\tau_{d}-1 .
$$

Em contrapartida, o filtro direto tem ação nos dois somatórios, dado que a resposta combinada $s_{f, k}(n-1), k=0, \ldots, M_{s}$ depende dos coeficientes do canal e do filtro direto. Desta forma, é possível entender porque o DFE pode apresentar um desempenho superior ao do LTE em canais com interferência intersimbólica severa.

2. Se $M_{b}>M_{s}-\tau_{d}-1$, a saída $y(n)$ é igual à (5.5) com um termo a mais dado por

$$
\sum_{k=M_{s}-\tau_{d}}^{M_{b}} w_{b, k}(n-1) a\left(n-\tau_{d}-k\right) .
$$

Neste caso, para eliminar esse termo e o segundo somatório de (5.5), os coeficientes dos filtro de realimentação devem satisfazer (5.6) e $w_{b, k}=0$, com $k=M_{s}-\tau_{d}, \ldots, M_{b}$. 
3. Se $M_{b}<M_{s}-\tau_{d}-1$ e os coeficientes do filtro de realimentação satisfizerem

$$
w_{b, k}(n-1)=-s_{f, k+\tau_{d}}(n-1)
$$

com $k=1, \ldots, M_{b}$, o somatório

$$
\sum_{k=M_{b}+1}^{M_{s}-\tau_{d}-1} s_{f, k+\tau_{d}} a\left(n-\tau_{d}-k\right)
$$

passa a ter influência apenas do filtro direto.

Desses resultados, conclui-se que a melhor escolha para o número de coeficientes do filtro de realimentação é $M_{b}=M_{s}-\tau_{d}-1$. Porém, o valor de $M_{s}$ é desconhecido na prática. Conseqüentemente, para escolher o valor de $M_{b}$ de forma sistemática deve-se iniciar $M_{b}$ com o valor de $M_{f}$ e ir aumentando até que o desempenho do DFE deixe de melhorar. Caso o desempenho não melhore para $M_{b} \geq M_{f}$, deve-se considerar novamente $M_{b}$ igual a $M_{f}$ e ir diminuindo até que o desempenho do DFE comece a piorar.

\subsection{O algoritmo que evita soluções degeneradas}

Quando algoritmos baseados no critério do módulo constante são utilizados para adaptação autodidata do DFE, podem ocorrer situações em que a saída $y(n)$ independe da entrada $u(n)$ e conseqüentemente da seqüência de símbolos transmitidos $a(n)$. São as chamadas soluções degeneradas [Papadias \& Paulraj, 1995; Szczecinski \& Gei, 2002]. Considerando por exemplo o DFE adaptado com o CMA e sinalização 4-QAM, os coeficientes dos filtros direto e de realimentação podem assumir a forma

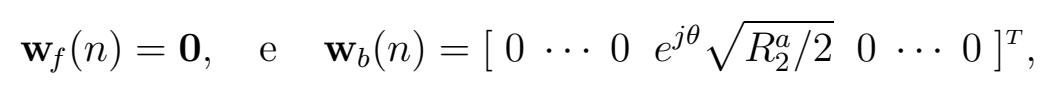

sendo $\theta$ uma fase arbitrária. Neste caso, o erro $e(n)=\left(|y(n)|^{2}-R_{2}^{a}\right) y(n)$ é nulo e a atualização dos coeficientes com o CMA deixa de ocorrer.

Para evitar esse problema, diferentes soluções foram propostas:

i) adaptação desacoplada dos filtros direto e de realimentação [Tong \& Liu, 1997; Labat et al., 1998];

ii) equalizador formado apenas pelo filtro de realimentação [Casas et al., 1995, 1999]; 
iii) adaptação conjunta dos filtros direto e de realimentação baseada na minimização da função custo de Godard com restrição [Papadias \& Paulraj, 1995; Szczecinski \& Gei, 2002].

Dentre essas soluções, as do tipo (iii) são mais eficientes, principalmente se o canal for variante no tempo [Szczecinski \& Gei, 2002]. Neste caso, destacam-se dois critérios: o proposto por Papadias e Paulraj (1995), que considera uma restrição apenas no filtro direto, levando ao algoritmo DFE-CMA-FF e o proposto por Szczecinski e Gei (2002), cuja restrição tem ação nos dois filtros, levando ao algoritmo denominado DFE-CMA-FB. Em [Szczecinski \& Gei, 2002], foi verificado que o DFE-CMA-FB é mais eficiente que o DFE-CMA-FF, dado que este último não consegue evitar todas as possíveis soluções degeneradas. Por esse motivo, apenas o DFE-CMA-FB é revisitado a seguir.

Supondo que $M_{b}=M_{s}-\tau_{d}-1$, para eliminação do segundo somatório de (5.5), a Equação (5.6) deve ser satisfeita. Isso implica

$$
\left\|\mathbf{w}_{b}(n-1)\right\|^{2}=\sum_{k=1}^{M_{b}}\left|w_{b, k}(n-1)\right|^{2}=\sum_{k=0}^{M_{s}-\tau_{d}-2}\left|s_{f, k}(n-1)\right|^{2} \leq \sum_{k=0}^{M_{s}-1}\left|s_{f, k}(n-1)\right|^{2} .
$$

Essa relação também pode ser estendida para $M_{b} \neq M_{s}-\tau_{d}-1$. Para $M_{b}>M_{s}-\tau_{d}-1$, além de (5.6), é necessário assumir que $w_{b, k}=0$, para $k=M_{s}-\tau_{d}, \ldots, M_{b}$.

Observando que

$$
\begin{aligned}
\mathrm{E}\left\{\left|y_{f}(n)\right|^{2}\right\} & =\sigma_{a}^{2} \sum_{k=0}^{M_{s}-1}\left|s_{f, k}(n-1)\right|^{2}+\mathrm{E}\left\{|v(n)|^{2}\right\} \\
& =\sigma_{a}^{2} \sum_{k=0}^{M_{s}-1}\left|s_{f, k}(n-1)\right|^{2}+\left\|\mathbf{w}_{f}(n-1)\right\|^{2} \sigma_{\eta}^{2},
\end{aligned}
$$

obtém-se a seguinte relação

$$
\sum_{k=0}^{M_{s}-1}\left|s_{f, k}(n-1)\right|^{2}=\frac{\mathrm{E}\left\{\left|y_{f}(n)\right|^{2}\right\}}{\sigma_{a}^{2}}-\left\|\mathbf{w}_{f}(n-1)\right\|^{2} \frac{\sigma_{\eta}^{2}}{\sigma_{a}^{2}}
$$

Substituindo (5.12) em (5.10), chega-se a

$$
\left\|\mathbf{w}_{b}(n-1)\right\|^{2} \leq \frac{\mathrm{E}\left\{\left|y_{f}(n)\right|^{2}\right\}}{\sigma_{a}^{2}}-\left\|\mathbf{w}_{f}(n-1)\right\|^{2} \frac{\sigma_{\eta}^{2}}{\sigma_{a}^{2}}
$$

Definindo a variável

$$
C_{w}(n)=\left\|\mathbf{w}_{b}(n-1)\right\|^{2}-\frac{\mathrm{E}\left\{\left|y_{f}(n)\right|^{2}\right\}}{\sigma_{a}^{2}}+\left\|\mathbf{w}_{f}(n-1)\right\|^{2} \frac{\sigma_{\eta}^{2}}{\sigma_{a}^{2}},
$$


(5.13) pode ser reescrita como $C_{w}(n) \leq 0$. Em [Szczecinski \& Gei, 2002, p. 1684], foi demonstrado por contradição que as soluções que satisfazem $C_{w}(n) \leq 0$ são não-degeneradas. Desta forma, deve-se minimizar a função custo de Godard sujeita a essa restrição, ou seja,

$$
J_{G_{\mathrm{DFE}}}=\left\{\mathrm{E}\left\{\left(|y(n)|^{2}-R_{2}^{a}\right)^{2}\right\} \mid C_{w}(n) \leq 0\right\}
$$

Utilizando o método dos multiplicadores de Lagrange [Haykin, 1996, p. 895], (5.15) pode ser reescrita como

$$
J_{G_{\mathrm{DFE}}}=\mathrm{E}\left\{\left(|y(n)|^{2}-R_{2}^{a}\right)^{2}\right\}+\lambda_{L} C_{w}(n) .
$$

A partir desse critério, obtém-se o algoritmo denominado DFE-CMA-FB, cujas operações são mostradas na Tabela 5.1. Nessa tabela, $0<\lambda_{f}<1$ é um fator de esquecimento e $\lambda_{L}(n)=l_{o} \operatorname{step}\left(C_{w}(n)\right)$ é o multiplicador de Lagrange, sendo

$$
\operatorname{step}(x)= \begin{cases}1, & \text { se } x \geq 0 \\ 0, & \text { caso contrário }\end{cases}
$$

a função degrau. É usual considerar $l_{o}=2$ e $\lambda_{f}=0.95$ [Szczecinski \& Gei, 2002]. Na obtenção do algoritmo, foram assumidos $\sigma_{a}^{2}=1 \mathrm{e}$

$$
\left\|\mathbf{w}_{b}(n-1)\right\|^{2}-\mathrm{E}\left\{\left|y_{f}(n)\right|^{2}\right\} \gg\left\|\mathbf{w}_{f}(n-1)\right\|^{2} \sigma_{\eta}^{2}
$$

Com essas considerações, a restrição se reduz a

$$
C_{w}(n) \approx\left\|\mathbf{w}_{b}(n-1)\right\|^{2}-\mathrm{E}\left\{\left|y_{f}(n)\right|^{2}\right\} \leq 0
$$

Desta forma, em cada iteração, deve-se calcular a norma dos coeficientes do filtro de realimentação e estimar a potência média da saída do filtro direto $\mathrm{E}\left\{\left|y_{f}(n)\right|^{2}\right\}$, o que é feito através de uma janela exponencial com um fator de esquecimento $\lambda_{f}$. A dedução desse algoritmo foi feita em [Szczecinski \& Gei, 2002].

Comparando os algoritmos DFE-CMA-FB e DFE-CMA (CMA convencional para adaptação do DFE), observa-se que quando a restrição é obedecida, o multiplicador de Lagrange é nulo e os dois algoritmos são idênticos. Caso contrário, $\lambda_{L}(n)=l_{o}$ e as equações de adaptação dos coeficientes dos filtros direto e de realimentação diferem. 


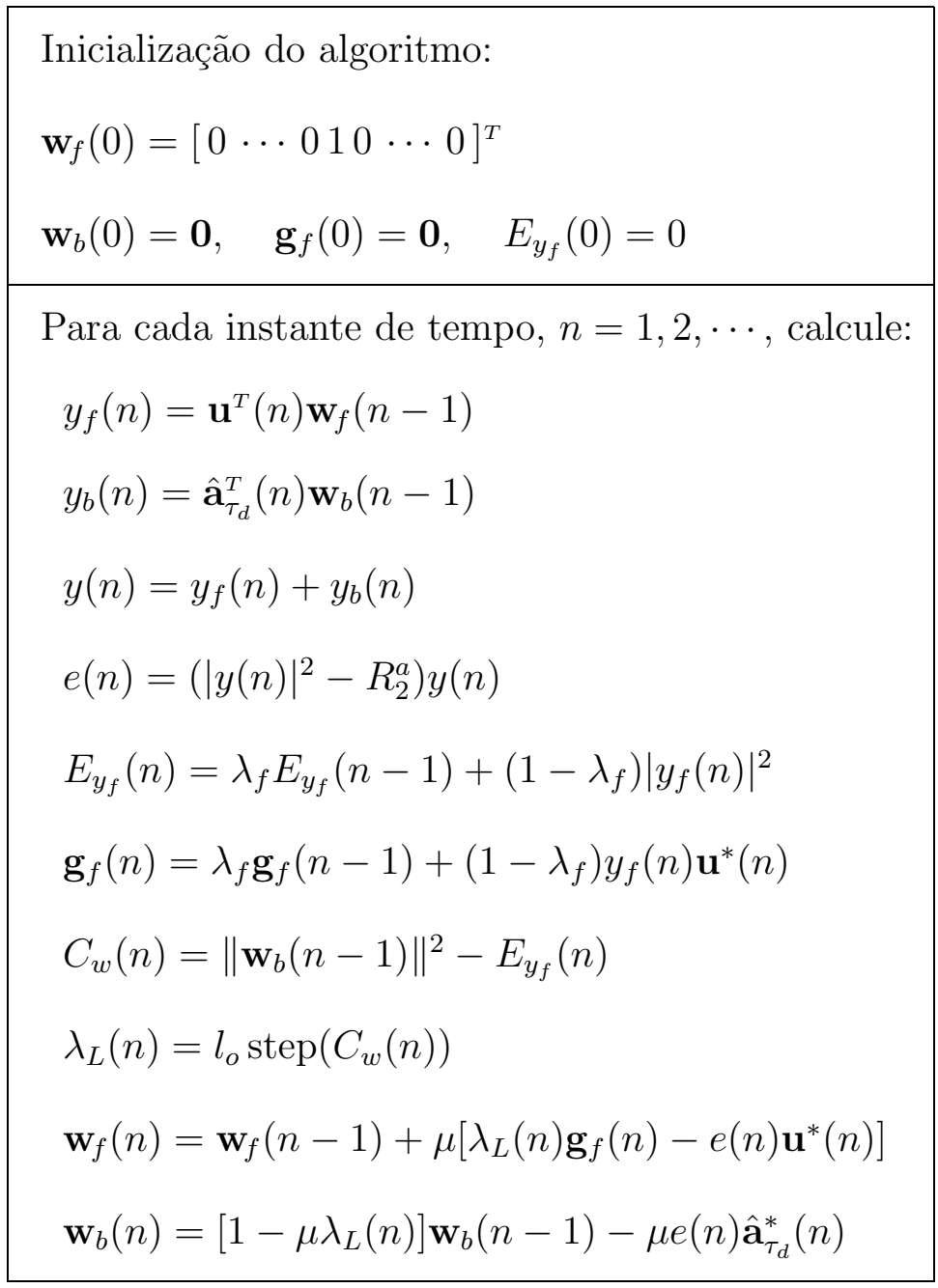

Tabela 5.1: Sumário do DFE-CMA-FB.

\subsection{O algoritmo concorrente para o DFE}

Dentre os algoritmos para adaptação autodidata do LTE e sinalização $M$-QAM, destaca-se o concorrente de Chen, revisitado no Apêndice D. Inspirando-se em seu bom comportamento, é proposto um algoritmo autodidata concorrente para adaptação do DFE, denominado NDEGSDD-CMA (Non-Degenerative SDD-CMA). Ele opera de forma concorrente o DFE-CMAFB com o SDD. Desta forma, espera-se que ele evite soluções degeneradas e adquira o bom comportamento dos algoritmos concorrentes. Na Figura 5.1, é mostrado um diagrama de blocos com as relações entre os algoritmos autodidatas para adaptação do LTE e do DFE, levando ao algoritmo proposto. As linhas tracejadas indicam que o DFE-CMA-FB foi inspirado no DFE-CMA-FF e o NDEG-SDD-CMA no CMA+SDD. As operações do algoritmo proposto são mostradas na Tabela 5.2. Elas consistem basicamente nas operações do DFE-CMA-FB da Tabela 5.1 adicionadas às do algoritmo SDD, revistado no Apêndice D. 


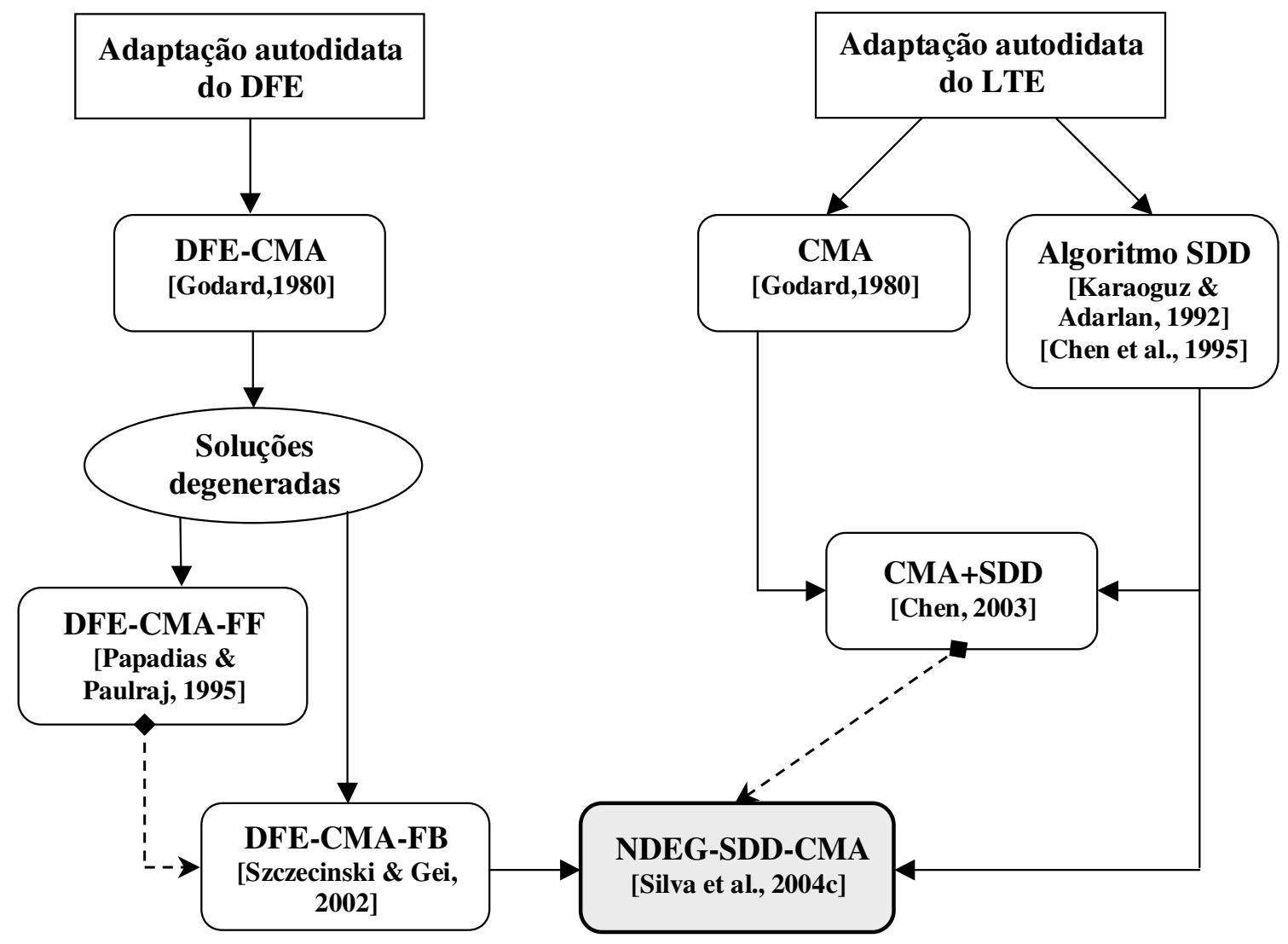

Figura 5.1: Relações entre os algoritmos para adaptação autodidata do DFE.

Cabe notar que $\mathbf{f}$ e b são respectivamente os vetores dos coeficientes dos filtros direto e de realimentação atualizados com o algoritmo SDD e $\mathbf{w}_{f}$ e $\mathbf{w}_{b}$ os vetores de coeficientes atualizados pelo DFE-CMA-FB.

Na Tabela 5.3, a complexidade computacional do NDEG-SDD-CMA é comparada às complexidades dos algoritmos DFE-CMA e DFE-CMA-FB. Considera-se, nas implementações do DFE-CMA e do DFE-CMA-FB, a compensação de fase antes do decisor com o PTA, revisitado na Seção 3.6, página 89. Embora o DFE-CMA não evite soluções degeneradas, ele é considerado aqui para simples comparação. A complexidade do NDEG-SDD-CMA foi calculada para constelação 4-QAM. No caso de uma constelação $M$-QAM, para identificar a região $A_{i}$, é necessário adicionar $2(L-1)$ comparações, sendo $L=\log _{2} \sqrt{M}$. Cabe notar que a complexidade computacional do algoritmo proposto é um pouco maior que a complexidade do DFE-CMA-FB. 


$$
\begin{aligned}
& \text { Inicialização do algoritmo: }
\end{aligned}
$$



$$
\begin{aligned}
& \mathbf{f}(0)=\mathbf{g}_{f}(0)=\mathbf{0}, \quad \mathbf{w}_{b}(0)=\mathbf{b}(0)=\mathbf{0}
\end{aligned}
$$

Para cada instante de tempo, $n=1,2, \ldots$, calcule:

$$
\begin{aligned}
& y_{f}(n)=\mathbf{u}^{T}(n)\left[\mathbf{w}_{f}(n-1)+\mathbf{f}(n-1)\right] \\
& y_{b}(n)=\hat{\mathbf{a}}_{\tau_{d}}^{T}(n)\left[\mathbf{w}_{b}(n-1)+\mathbf{b}(n-1)\right] \\
& y(n)=y_{f}(n)+y_{b}(n) \\
& e(n)=\left(|y(n)|^{2}-R_{2}^{a}\right) y(n) \\
& E_{y_{f}}(n)=\lambda_{f} E_{y_{f}}(n-1)+\left(1-\lambda_{f}\right)\left|y_{f}(n)\right|^{2} \\
& \mathbf{g}_{f}(n)=\lambda_{f} \mathbf{g}_{f}(n-1)+\left(1-\lambda_{f}\right) y_{f}(n) \mathbf{u}^{*}(n) \\
& C_{w}(n)=\left\|\mathbf{w}_{b}(n-1)\right\|^{2}-E_{y_{f}}(n) \\
& \lambda_{L}(n)=l_{o} \operatorname{step}\left(C_{w}(n)\right) \\
& \mathbf{w}_{f}(n)=\mathbf{w}_{f}(n-1)+\mu\left[\lambda_{L}(n) \mathbf{g}_{f}(n)-e(n) \mathbf{u}^{*}(n)\right] \\
& \mathbf{w}_{b}(n)=\left[1-\mu \lambda_{L}(n)\right] \mathbf{w}_{b}(n-1)-\mu e(n) \hat{\mathbf{a}}_{\tau_{d}}^{*}(n)
\end{aligned}
$$

Identifique a região $A_{i}$ fazendo $2(L-1)$

comparações e calcule:

$$
\begin{aligned}
\xi(n) & =\frac{\sum_{m=1}^{4} \exp \left[-\frac{\left|\varepsilon_{i m}(n)\right|^{2}}{2 \tilde{\rho}}\right] \varepsilon_{i m}(n)}{\sum_{m=1}^{4} \exp \left[-\frac{\left|\varepsilon_{i m}(n)\right|^{2}}{2 \tilde{\rho}}\right]} \\
\mathbf{f}(n) & =\mathbf{f}(n-1)-\mu_{d} \xi(n) \mathbf{u}_{f}^{*}(n) \\
\mathbf{b}(n) & =\mathbf{b}(n-1)-\mu_{d} \xi(n) \hat{\mathbf{a}}_{\tau_{d}}^{*}(n)
\end{aligned}
$$

Tabela 5.2: Sumário do NDEG-SDD-CMA.

\begin{tabular}{|c|c|c|c|}
\hline Op. & DFE-CMA+PTA & DFE-CMA-FB+PTA & NDEG-SDD-CMA \\
\hline$\times$ & $8 M_{f}+8 M_{b}+19$ & $16 M_{f}+12 M_{b}+28$ & $20 M_{f}+16 M_{b}+36$ \\
\hline+ & $8 M_{f}+8 M_{b}+7$ & $14 M_{f}+10 M_{b}+11$ & $20 M_{f}+16 M_{b}+25$ \\
\hline$\div$ & - & - & 1 \\
\hline $\exp$ & - & - & 4 \\
\hline
\end{tabular}

Tabela 5.3: Complexidade computacional em termos de operações complexas por iteração. 


\subsection{A rede neural recorrente}

Uma rede neural recorrente (RNN - Recurrent Neural Network) com $N=3$ neurônios totalmente interconectados e $M$ entradas externas está esquematizada na Figura 5.2. As entradas externas podem ser agrupadas no seguinte vetor

$$
\mathbf{x}(n)=\left[x_{0}(n) x_{1}(n) \cdots x_{M-1}(n)\right]^{T} .
$$

No caso da equalização, esse vetor é formado por $M$ amostras atrasadas do sinal $u(n)$ [Kechriotis et al., 1994a]. A saída de cada neurônio no instante $n+1$ depende de $\mathbf{x}(n)$ e das saídas de todos os neurônios no instante de tempo anterior, ou seja, $y_{k}(n), 1 \leq k \leq N$. Na Figura 5.2, os atrasos existentes nas conexões entre os neurônios e a função de ativação nas saídas dos neurônios 1 e 2 foram omitidos. O neurônio de saída (neurônio 3, neste caso) é uma unidade linear. A dinâmica da RNN é descrita pelas seguintes equações

$$
\begin{aligned}
& v_{k}(n+1)=\sum_{l=1}^{N} w_{k l}(n) y_{l}(n)+\sum_{l=0}^{M-1} w_{k l^{\prime}}(n) x_{l}(n), \\
& y_{k}(n+1)= \begin{cases}v_{k}(n+1), & k=i \\
\chi\left(v_{k}(n+1)\right), & k \neq i,\end{cases}
\end{aligned}
$$

sendo $l^{\prime}=l+N+1, y_{k}(n)$ a saída do $k$-ésimo neurônio, $i$ o neurônio de saída, $\chi(v)=$ $\varphi\left(v_{R}\right)+j \varphi\left(v_{I}\right)$ a função de ativação com $v_{R}$ e $v_{I}$ as partes real e imaginária de $v$ e $\varphi(\cdot)$ a função sigmoidal [Kechriotis et al., 1994a; Haykin, 1999].

O algoritmo RTRL (Real Time Recurrent Learning), revisitado no Apêndice E, é o mais conhecido para o treinamento da RNN [Zisper \& Williams, 1991; Haykin, 1999; Kechriotis et al., 1994a]. Ele também pode ser usado na adaptação autodidata dos coeficientes [Kechriotis et al., 1992]. Neste caso, o erro do $k$-ésimo neurônio, denotado por $e_{k}(n)$, é baseado no critério do módulo constante [Godard, 1980], isto é,

$$
e_{k}(n)= \begin{cases}\left(\left|y_{k}(n)\right|^{2}-R_{2}^{a}\right) y_{k}(n), & k=i \\ 0, & k \neq i\end{cases}
$$

sendo $i$ o neurônio de saída.

A adaptação autodidata da RNN baseada no critério do Módulo Constante não garante que um bom desempenho na equalização seja atingido [Kechriotis et al., 1992]. Além disso, foi verificado que quando se aumenta o número de neurônios, sérios problemas relacionados 


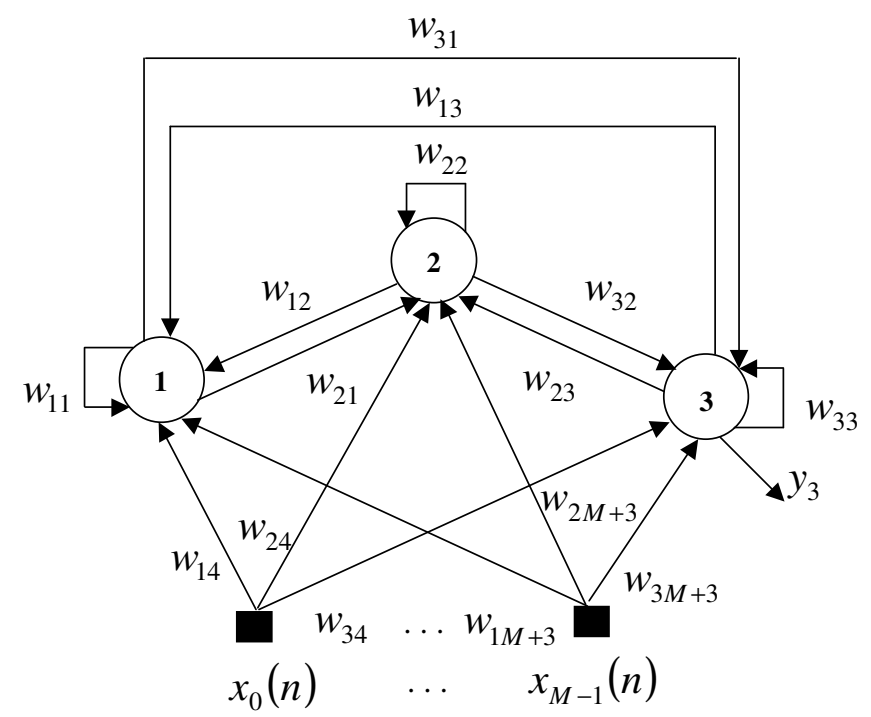

Figura 5.2: RNN com $N=3$ neurônios, $M$ entradas e saída $y_{3}$.

a mínimos locais podem aparecer [Gomes \& Barroso, 1995]. Esses mínimos são associados à não-linearidade do algoritmo autodidata adicionada às não- linearidades da rede. Entretanto, se a RNN for utilizada na malha de realimentação do DFE, o critério do módulo constante pode ser considerado. Neste caso, verificou-se através de simulações que essa rede apresenta um bom desempenho.

\subsection{O equalizador híbrido}

Embora o DFE seja amplamente utilizado na prática, ele pode sofrer propagação de erros devido a decisões incorretas. Tentando reduzir esse problema, utiliza-se a RNN no processamento das decisões antes da realimentação. Esse esquema, originalmente proposto em [Silva, 2001; Silva \& Gerken, 2002a] para o caso supervisionado, pode ser estendido para equalização autodidata [Silva et al., 2004b], como mostrado na Figura 5.3. O equalizador híbrido é formado por dois combinadores lineares (LCs - Linear Combiners) e uma RNN, formando uma estrutura de DFE. Por isso, ele também pode ser chamado de DFE híbrido. O filtro direto é chamado de FF-LC (feedforward-LC) e tem como entrada $M_{f}$ amostras atrasadas do sinal $u(n)$. O filtro de realimentação é formado por um combinador linear de realimentação FB-LC (feedback-LC) e uma RNN. A entrada do FB-LC é dada por $M_{r}$ amostras atrasadas da saída da RNN. A entrada da RNN é formada pelas mesmas $M_{f}$ amostras de $u(n)$ e $M_{b}$ 
decisões passadas que são realimentadas, isto é, o vetor de entrada da RNN tem dimensão $M_{f}+M_{b}$ e é dado por

$$
\mathbf{x}(n)=\mathbf{u}_{f b}(n)=\left[\mathbf{u}^{T}(n) \hat{\mathbf{a}}_{\tau_{d}}^{T}(n)\right]^{T}
$$

com $\mathbf{u}(n)$ e $\hat{\mathbf{a}}_{\tau_{d}}(n)$ definidos respectivamente em (1.27) e (1.29), página 17.

A adaptação autodidata em tempo real do equalizador híbrido pode ser feita utilizando-se o algoritmo RTRL, revisitado no Apêndice E, para a atualização dos coeficientes da RNN e o DFE-CMA-FB para a dos coeficientes dos LCs. Cabe observar que a adaptação autodidata desse esquema também pode convergir para soluções degeneradas. Por essa razão, o DFECMA-FB foi escolhido para adaptar os coeficientes dos LCs.

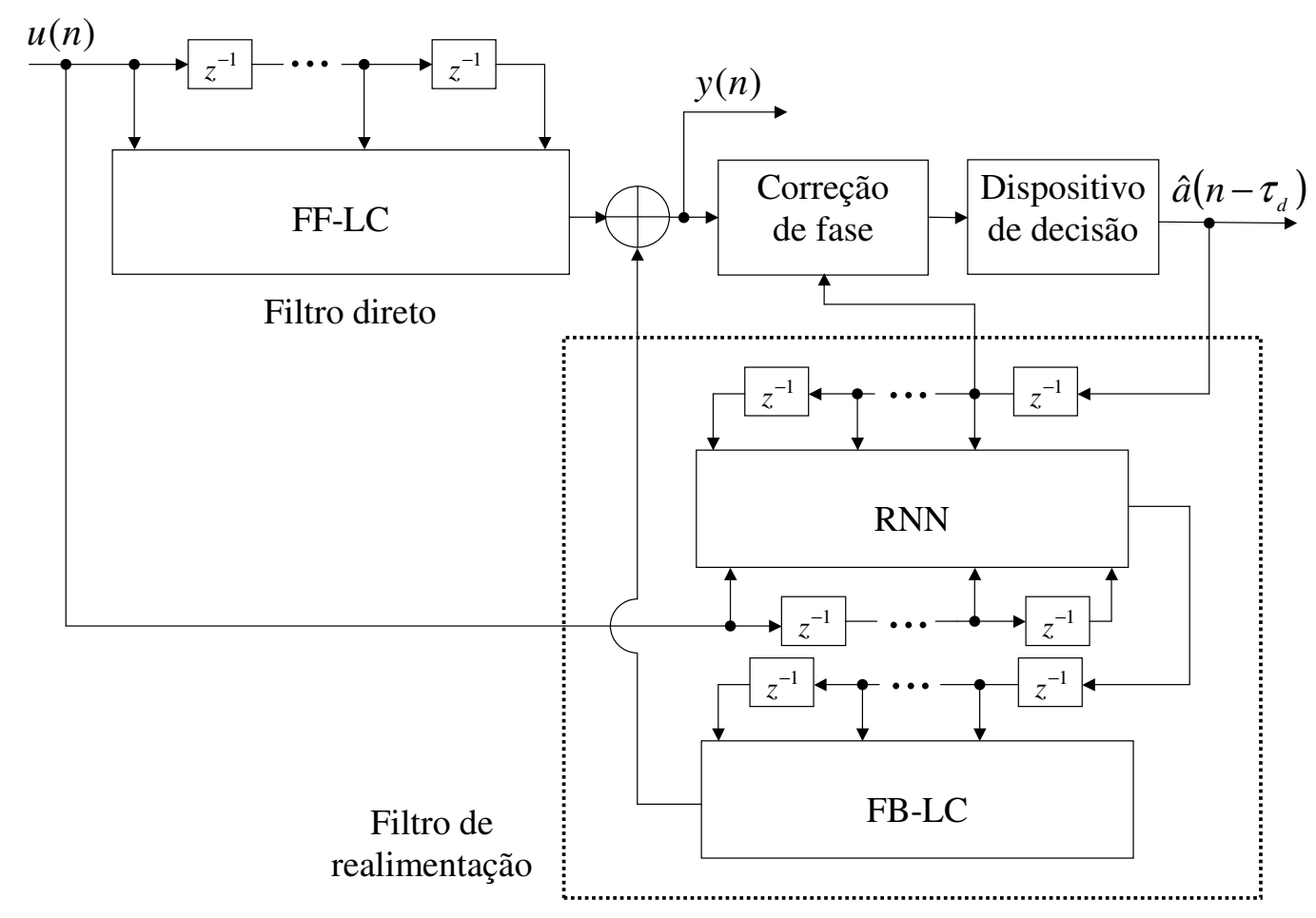

Figura 5.3: Esquema do equalizador híbrido RNN-LC.

O problema que aparece é como obter o erro na saída da RNN, ou em outras palavras, como propagar o erro da saída dos LCs para suas entradas. Felizmente, devido à natureza linear dos LCs, esse problema tem uma solução simples, mostrada na Figura 5.4. Supõe-se inicialmente que a RNN não tenha erro e que o sinal $y(n)$ seja o sinal desejado $\hat{a}\left(n-\tau_{d}\right)$ (Figura 5.4-a). Em seguida, assume-se que há um erro $e_{\mathrm{RNN}}(n)$ na saída da RNN, obtendose desta forma o sinal $y(n)=\hat{a}\left(n-\tau_{d}\right)-e(n)$ (Figura 5.4-b). Finalmente, subtraindo os esquemas das figuras 5.4-a e 5.4-b, chega-se à Figura 5.4-c e o erro e(n) pode ser descrito 
pela expressão

$$
e(n)=\sum_{k=1}^{M_{r}} w_{b, k}(n-1) e_{\mathrm{RNN}}(n-k+1),
$$

sendo $w_{b, k}, k=1,2, \ldots, M_{r}$, os coeficientes do FB-LC. A partir de (5.21), pode-se calcular o erro da RNN como

$$
e_{\mathrm{RNN}}(n)=\frac{e(n)-\sum_{k=1}^{M_{r}} w_{b, k}(n-1) e_{\mathrm{RNN}}(n-k+1)}{w_{b, 1}(n-1)} .
$$

De (5.22), conclui-se que $w_{b, 1}(n-1) \neq 0$ deve ser satisfeito. Para garantir essa condição, $w_{b, 1}(n-1)=1$ foi imposto. Além disso, a partir de simulações, verificou-se que uma outra simplificação possível é dada por $e_{\mathrm{RNN}}(n)=e(n)$, o que não introduz mudanças significativas no desempenho do equalizador. Cabe observar que a complexidade computational do algoritmo de treinamento é a adição das complexidades do DFE-CMA-FB para o treinamento dos LCs e do algoritmo RTRL autodidata para o treinamento da RNN.

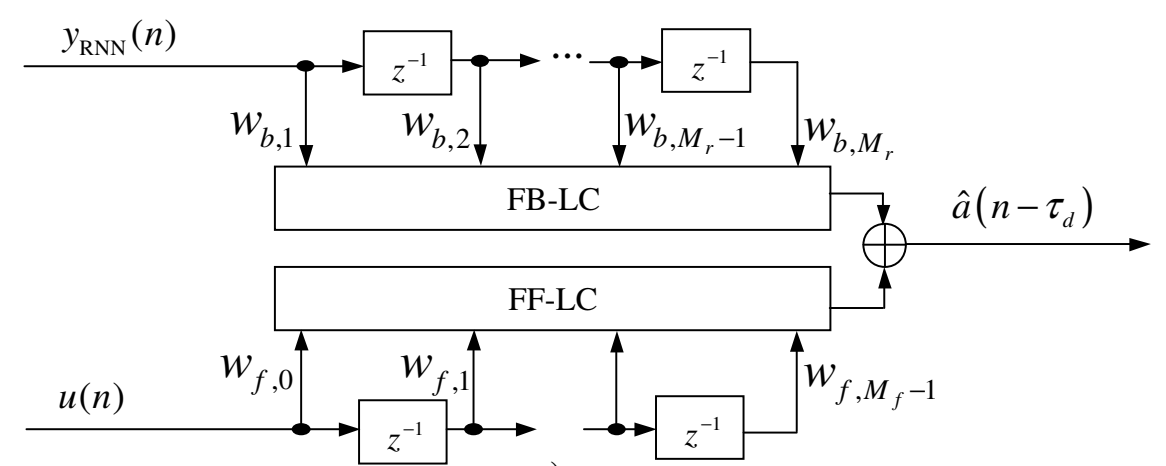

a)

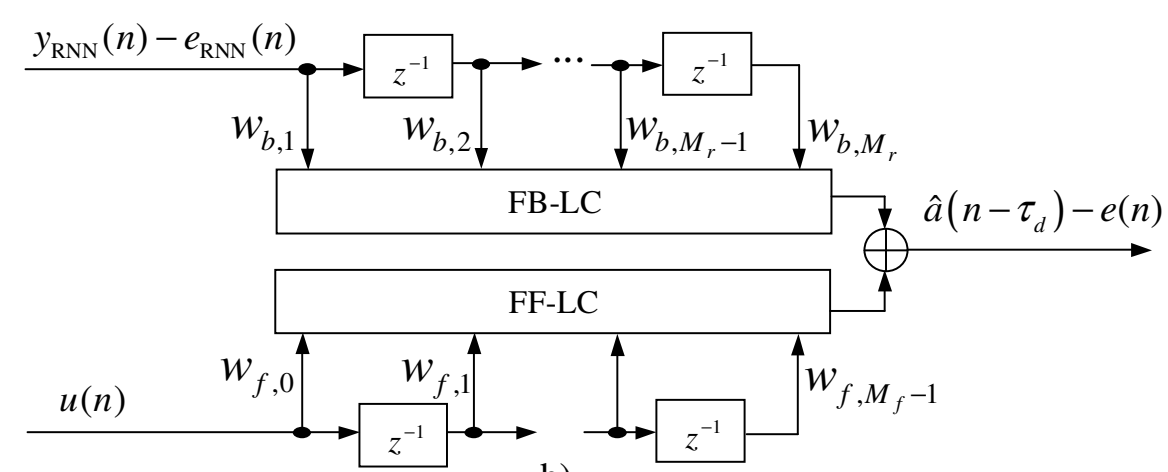

b)

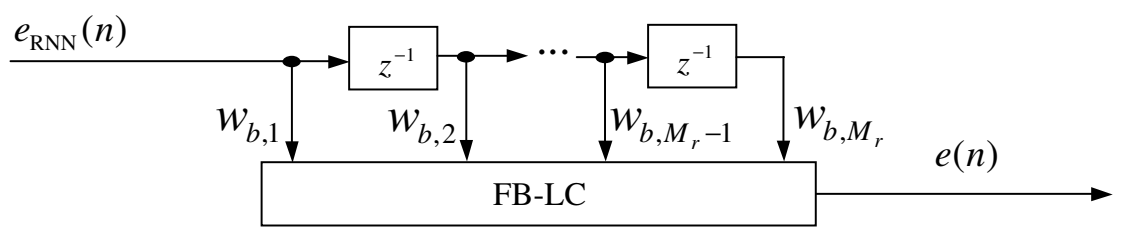

c)

Figura 5.4: Propagação de erro para adaptação da RNN. 


\subsection{Resultados experimentais}

Nesta seção, são apresentados alguns resultados de simulações com o DFE, adaptado de forma autodidata. Inicialmente, o algoritmo concorrente NDEG-SDD-CMA é comparado com o não-concorrente DFE-CMA-FB. Em seguida, o equalizador híbrido é comparado com a RNN e o DFE autodidatas.

Cabe observar que em equalizadores não-lineares, a ISI residual deixa de ser um medida de desempenho adequada. No caso do DFE, deve-se conhecer exatamente o atraso da recuperação do sinal transmitido. No caso da RNN e do DFE híbrido, não é mais possível calcular a ISI de forma simples. Por isso, consideram-se alternativamente outras medidas como o erro quadrático médio (MSE) baseado na decisão e a taxa de erro de símbolo (SER - Symbol Error Rate). O MSE baseado na decisão é definido como

$$
\mathrm{MSE}_{\mathrm{dec}}=\mathrm{E}\left\{\left|y(n)-\hat{a}\left(n-\tau_{d}\right)\right|^{2}\right\}
$$

Neste caso, compara-se o sinal antes e depois do decisor, calculando-se o erro de decisão, o que pode dar uma idéia da velocidade de convergência dos algoritmos autodidatas. A SER é calculada, comparando-se a seqüência transmitida com a seqüência de saída do decisor. Para isso, após a convergência, os coeficientes do equalizador são "congelados" e uma seqüência de símbolos é transmitida, a fim de que o número de erros para uma dada relação sinal-ruído seja igual ou maior que 30. Cabe observar que o comprimento da seqüência de símbolos utilizada depende da relação sinal-ruído, quando maior o valor de SNR maior a quantidade de símbolos. Para canais variantes no tempo, a SER é calculada instantaneamente.

Para compensar uma eventual rotação de fase não-múltipla de $\pi / 2$, o PTA é considerado nas implementações dos algoritmos RTRL autodidata e DFE-CMA-FB. No algoritmo concorrente, a utilização do PTA não é mais necessária, dado que ele é capaz de corrigir esse tipo de rotação.

\subsubsection{Algoritmo concorrente para o DFE}

Considera-se um canal de TV digital (DTV - Digital Television) de uma transmissão metropolitana terrestre [Ghosh, 1998]. Neste caso, o sinal é recebido por um percurso direto entre as antenas de transmissão e recepção e também por reflexões de outros objetos como prédios 
e/ou morros próximos do receptor. Este cenário é comum em grandes metrópoles, como por exemplo, Nova Iorque, Tóquio e São Paulo. Os coeficientes e a resposta em freqüência do canal são mostrados na Figura 5.5. Nas simulações, o sinal transmitido e o sinal de entrada do equalizador foram normalizados para que tivessem potência unitária, isto é, $\sigma_{a}^{2}=\sigma_{u}^{2}=1$. Essa normalização foi considerada para possibilitar a simplificação da Equação (5.18) e conseqüentemente, para que o algoritmo da Tabela 5.1 fosse utilizado sem modificação.
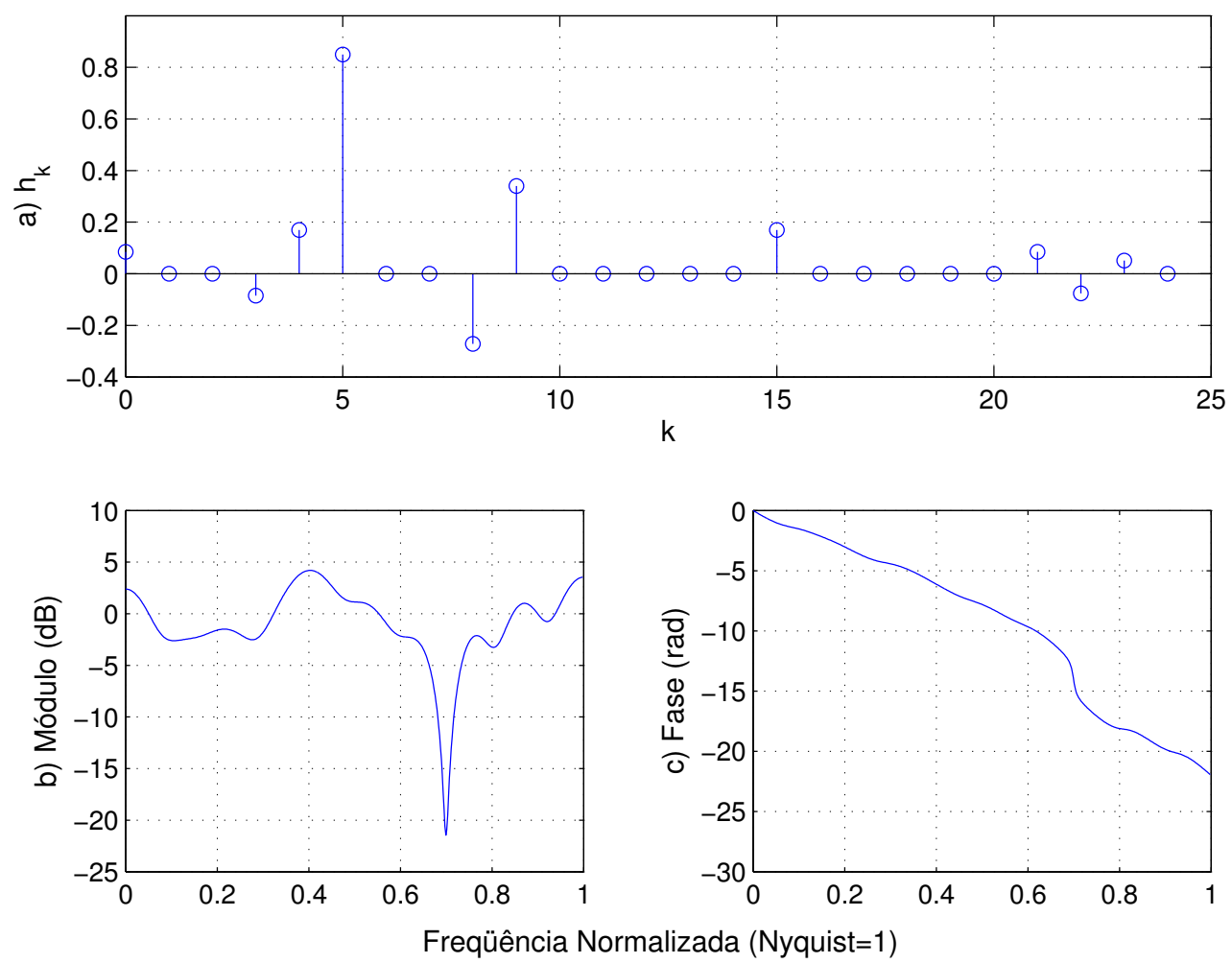

Figura 5.5: a) Resposta ao pulso unitário, b) e c) Resposta em freqüência do canal $\mathrm{H}_{15}$.

O desempenho do NDEG-SDD-CMA é comparado ao do DFE-CMA-FB e ao do algoritmo DFE-LMS sem propagação de erros (SPE) para o treinamento do DFE com $M_{f}=11$, $M_{b}=17$ e sinalização 16-QAM. Na Figura 5.6, são mostradas curvas de taxa de erro de símbolo (SER - Symbol Error Rate). Como esperado, o algoritmo supervisionado DFE-LMS (SPE) apresenta o melhor desempenho. O NDEG-SDD-CMA apresenta um comportamento próximo do DFE-CMA-FB para uma relação sinal-ruído (SNR) abaixo de 19 dB, mas é melhor que ele para SNR > 22 dB. Desta forma, o algoritmo proposto apresenta um desempenho próximo ao do DFE-CMA-FB para SNR baixa e é melhor que ele para SNR alta. 


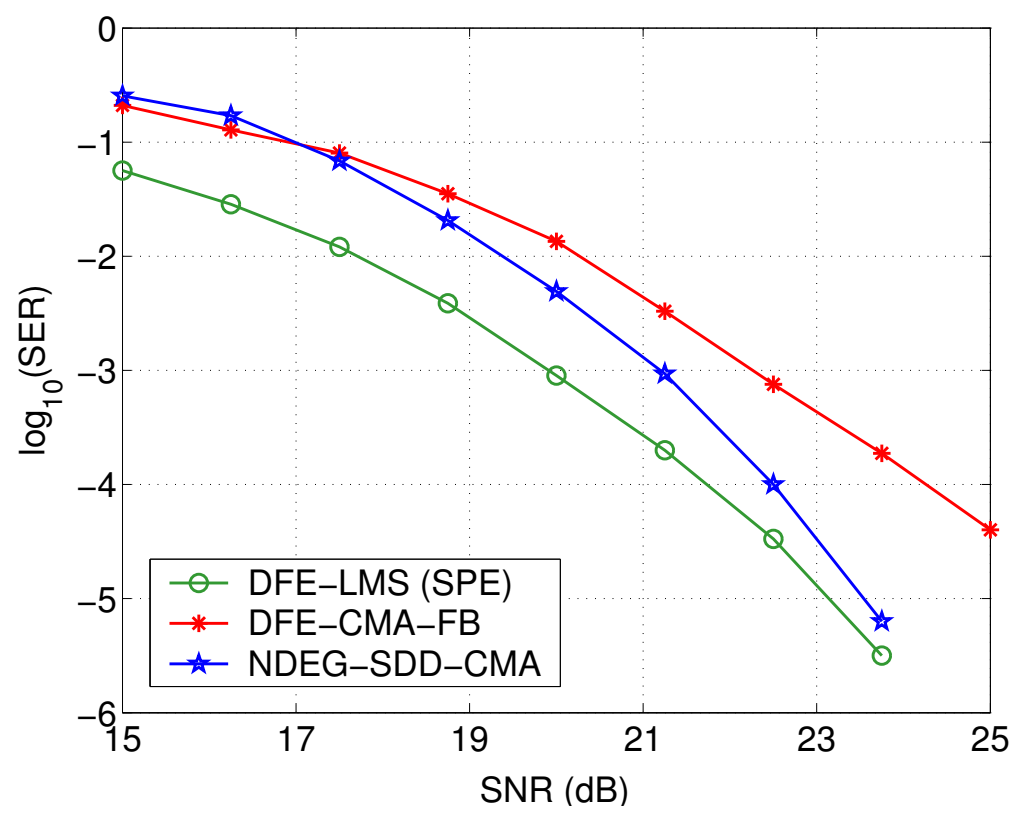

Figura 5.6: Curvas de taxa de erro de símbolo (SER) em função da relação sinal ruído (SNR) para 16-QAM, $M_{f}=11, M_{b}=17$, canal $\mathrm{H}_{15}, \sigma_{d}=10, \mu=10^{-3}, \mu_{d}=10^{-2}, \mu_{\theta}=10^{-2}, \tilde{\rho}=0,06$.

Na Figura 5.7, são mostradas curvas de erro quadrático médio baseado na decisão, considerando o NDEG-SDD-CMA, o DFE-CMA-FB e o DFE-LMS (SPE) para $\mathrm{SNR}=22,5 \mathrm{~dB}$ e 16-QAM. Para facilitar a visualização, os sinais de erro foram filtrados por um filtro média móvel com 32 coeficientes. Os algoritmos foram ajustados experimentalmente para atingir o mesmo MSE em regime. Cabe observar que o NDEG-SDD-CMA apresenta uma convergência mais rápida que o DFE-CMA-FB e próxima do algoritmo DFE-LMS (SPE).

A fim de investigar o desempenho dos algoritmos em um canal de TV digital com multipercurso não-estacionário, considera-se o canal

$$
\mathrm{H}_{16}(z)=1+0,5 \operatorname{sen}(2 \pi f n T) z^{-12}
$$

sendo $f=5 \mathrm{~Hz}, T=0.0929 \mathrm{~ms}$ e $n$ o instante de tempo [Ghosh, 1998]. Na Figura 5.8, são mostrados os erros na saída do decisor para 16-QAM, SNR $=20 \mathrm{~dB}, M_{f}=11 \mathrm{e}$ $M_{b}=17$. O passo de adaptação de cada algoritmo foi experimentalmente ajustado para se obter a melhor capacidade de tracking. Os valores absolutos das raízes (zeros) do canal são mostrados na Figura 5.8-d. O canal tem 11 zeros com o mesmo valor absoluto, distribuídos uniformemente numa circunferência. O canal se torna mais difícil de equalizar quando os zeros estão próximos da circunferência unitária. Neste caso, o DFE-CMA-FB apresenta 
erros em seqüência (Figura 5.8-c), enquanto o algoritmo DFE-LMS (SPE) (Figura 5.8-a) e o NDEG-SDD-CMA (Figura 5.8-b) apresentam recuperações mais rápidas.



Figura 5.7: MSE baseado na decisão, média de 50 realizações, 16 -QAM, canal $\mathrm{H}_{15}, \mathrm{SNR}=22,5 \mathrm{~dB}$, $M_{f}=11$ e $M_{b}=17 ;$ LMS-DFE $(\mathrm{SPE})\left(\mu=2,5 \times 10^{-3}, \tau_{d}=10\right)$, DFE-CMA-FB $\left(\mu=2,5 \times 10^{-4}\right)$ e NDEG-SDD-CMA $\left(\mu=10^{-3}, \mu_{d}=10^{-2}, \tilde{\rho}=0,06\right)$.

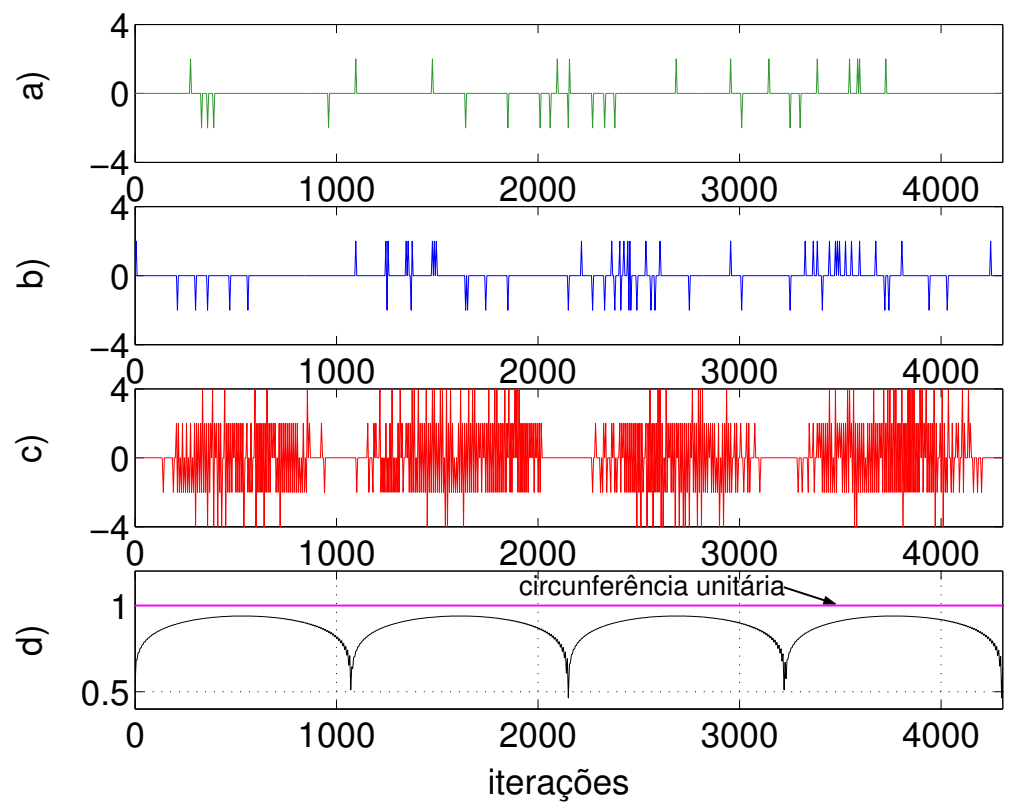

Figura 5.8: Erros na recuperação do sinal para a) DFE-LMS (SPE) $\left(\mu=10^{-2}, \sigma_{d}=5\right)$, b) NDEG-SDD-CMA $\left(\mu=2 \times 10^{-3}, \mu_{d}=2 \times 10^{-2}, \tilde{\rho}=0,06\right)$, c) DFE-CMA-FB $\left(\mu=5 \times 10^{-4}\right.$, $\tilde{\rho}=0,06, \mu_{\theta}=5 \times 10^{-3}$ ); d) Módulos dos zeros do canal $\mathrm{H}_{16}$ ao longo das iterações; $M_{f}=11$, $M_{b}=17, \mathrm{SNR}=20 \mathrm{~dB}, 16-\mathrm{QAM}$. 
Na Figura 5.9, são mostradas curvas de SER para o canal $\mathrm{H}_{16}$. Devido aos erros em seqüência, o DFE-CMA-FB apresenta valores de taxa de erro de símbolo elevados. O algoritmo DFE-LMS apresenta o melhor desempenho seguido pelo NDEG-SDD-CMA. Entretanto, este último sofre com baixos valores de SNR. Para SNR $<17,5 \mathrm{~dB}$ seu desempenho se torna pior que o do DFE-CMA-FB. Como observado anteriormente, o bom desempenho do algoritmo proposto é obtido para altos valores de SNR.

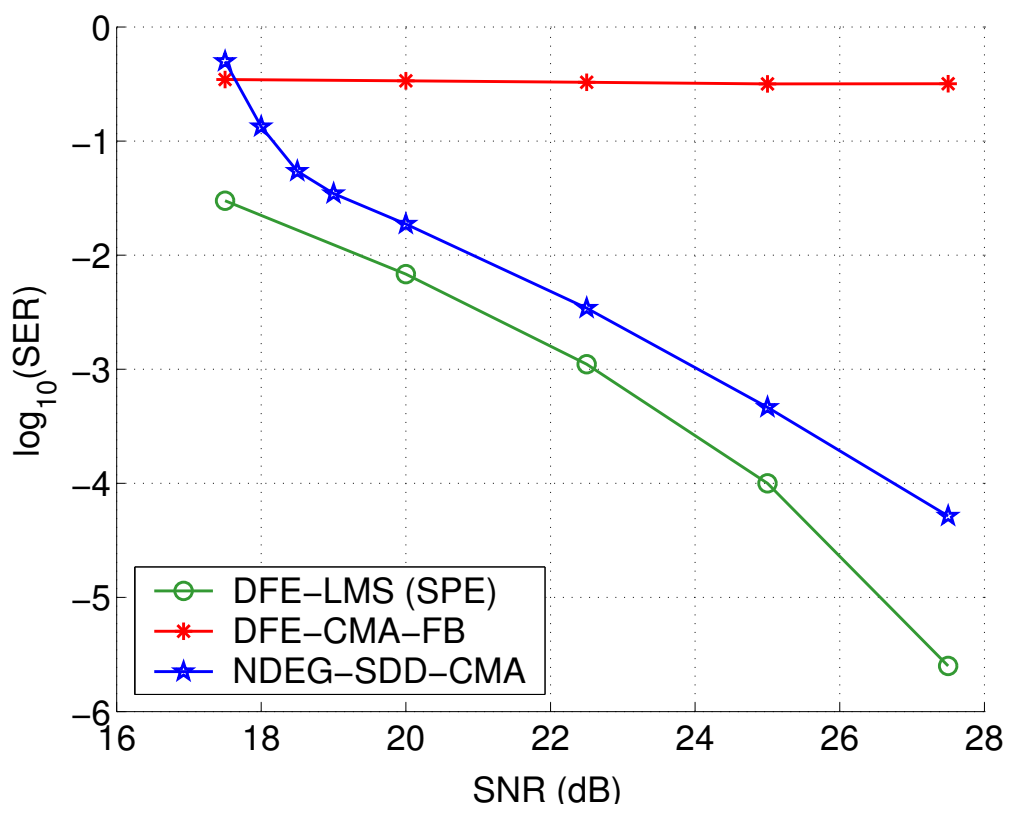

Figura 5.9: Curvas de taxa de erro de símbolo (SER) em função da relação sinal ruído (SNR) para $M_{f}=11, M_{b}=17$, canal $\mathrm{H}_{16}$ e 16-QAM.

\subsubsection{DFE híbrido}

Os modelos dos canais usados nas simulações estão mostrados na Tabela 5.4. Além desses, considera-se também o canal variante no tempo (canal $\mathrm{H}_{8}$ ), cujos coeficientes estão mostrados na Figura 3.11 da página 97.

Na Figura 5.10, são mostradas curvas de MSE baseado na decisão para o DFE, a RNN e o DFE híbrido, adaptados de forma autodidata. A fim de facilitar a visualização, os sinais de MSE foram filtrados por um filtro média móvel com 64 coeficientes. O DFE híbrido apresenta a convergência mais rápida, seguido da RNN e do DFE. Cabe observar que a RNN apresenta uma convergência abrupta, mas precisa de $2 \times 10^{4}$ iterações para alcançar o MSE mínimo. O DFE híbrido adquire o comportamento abrupto da RNN, mas atinge o mínimo com apenas 5000 iterações. 


\begin{tabular}{|l|}
$\mathrm{H}_{17}$ (Fase não-mínima) \\
$\mathrm{H}_{17}(z)=(0,429-j 0,064)+(0,538+j 0,807) z^{-1}+(0,429-j 0,064) z^{-2}$ \\
$\mathrm{H}_{18}\left(\mathrm{H}_{17}\right.$ com não-linearidades $)$ \\
$x_{l}(n)=\mathrm{H}_{17}(z) a(n)$ \\
$x(n)=x_{l}(n)+0,1 x_{l}^{2}(n)+0,05 x_{l}^{3}(n)$ \\
\hline \hline Notação: $z^{-1} a(n)=a(n-1)$ \\
$a(n):$ entrada do canal \\
$x(n):$ saída do canal sem ruído
\end{tabular}

Tabela 5.4: Modelos dos canais utilizados nas simulações com o equalizador híbrido.

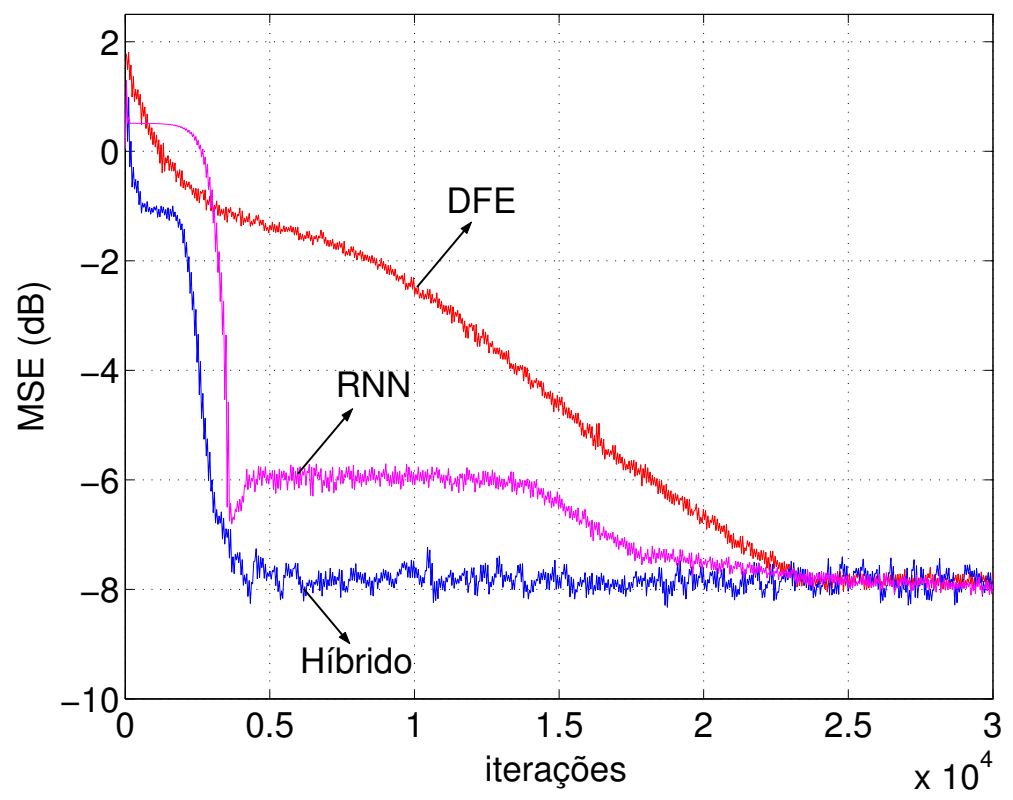

Figura 5.10: $\mathrm{MSE}$ baseado na decisão, média de 50 realizações, 16 -QAM, canal $\mathrm{H}_{17}, \mathrm{SNR}=25 \mathrm{~dB}$, DFE-CMA-FB $\left(M_{f}=5, M_{b}=4, \mu=10^{-4}\right)$, RNN-RTRL $\left(M=5, N=3, \mu_{\mathrm{RTRL}}=10^{-3}\right)$, Híbrido $\left(M_{f}=5, M_{b}=M_{r}=4, N=3, \mu=10^{-4}, \mu_{\mathrm{RTRL}}=10^{-3}\right), \mu_{\theta}=10^{-3}$.

Na Figura 5.11, são mostradas curvas de taxa de erro de símbolo (SER) para os equalizadores, considerando um canal não-linear $\left(\mathrm{H}_{18}\right)$ e sinalização 4-QAM. O equalizador linear transversal (LTE) treinado com o CMA apresenta o pior desempenho, o que era esperado, já que equalizadores não-lineares nem sempre funcionam adequadamente quando há nãolinearidades nos canais de transmissão. A RNN e o DFE autodidatas apresentam desempenhos próximos, sendo o DFE ligeiramente melhor para $\mathrm{SNR}>17,5 \mathrm{~dB}$. Para esse canal, 
o DFE híbrido mostra sua superioridade, tendo um desempenho próximo da solução ótima baseada no critério de Bayes [Proakis, 1996; Mulgrew, 1996]. Cabe observar que para se obter a solução ótima de Bayes, a realimentação de decisões e o conhecimento do modelo do canal foram considerados.

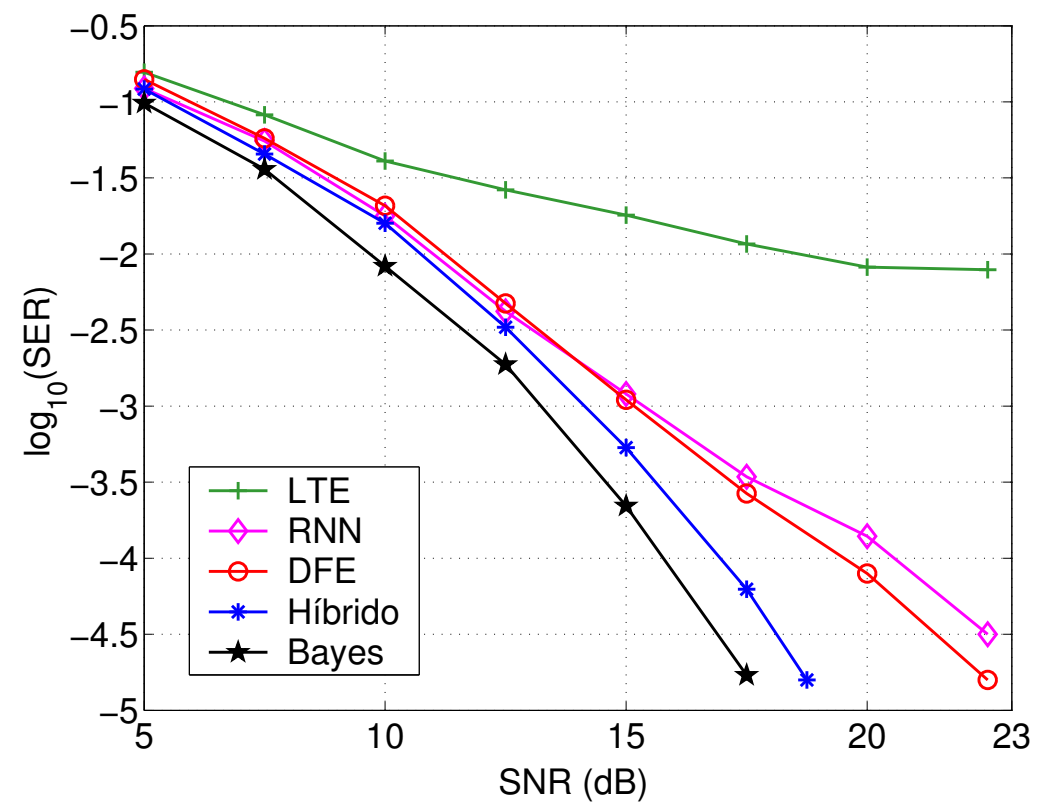

Figura 5.11: Curvas de taxa de erro de símbolo (SER) em função da relação sinal ruído (SNR) para LTE $(M=5), \operatorname{RNN}(M=3, N=3)$, DFE $\left(M_{f}=3, M_{b}=2\right)$, Híbrido $\left(M_{f}=3, M_{b}=M_{r}=2\right.$, $N=3)$, 4-QAM e canal $\mathrm{H}_{18}$.

Na Figura 5.12, são mostrados os erros na saída do decisor, considerando o canal linear e variante no tempo (canal $\mathrm{H}_{8}$ ), $\mathrm{SNR}=25 \mathrm{~dB}$ e 4-QAM. Os valores absolutos das raízes do polinômio $h_{0}(n) x^{2}+h_{1}(n) x+h_{2}(n)$ estão mostrados na Figura 5.12-d. Desta forma, os erros em seqüência podem ser associados aos nulos espectrais pronunciados do canal. Em todas essas situações, o DFE híbrido apresenta uma recuperação mais rápida. A RNN autodidata não converge adequadamente para esse canal. Em contrapartida, ela possui um papel importante no processamento das decisões passadas, fazendo com que o DFE híbrido apresente uma boa capacidade de tracking. 


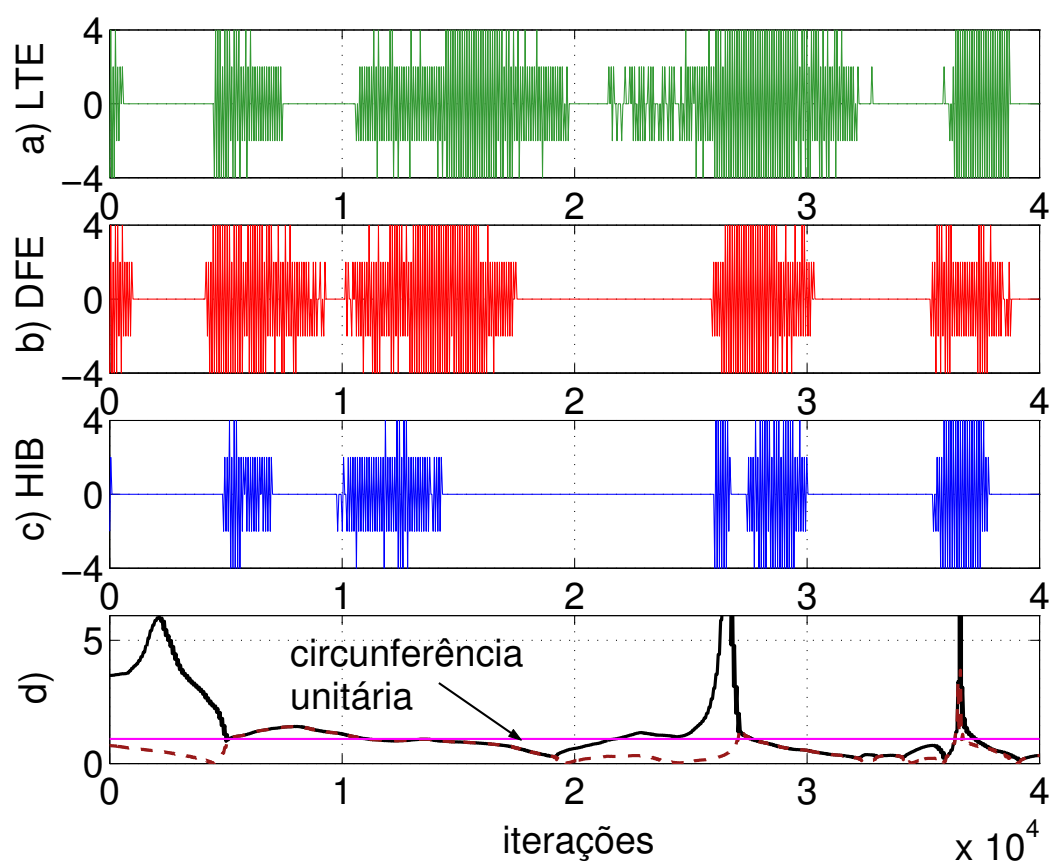

Figura 5.12: a), b) e c) Erros na recuperação do sinal para a) $\operatorname{LTE}-\operatorname{CMA}\left(M=9, \mu=10^{-3}\right)$, b) DFE-CMA-FB $\left(M_{f}=5, M_{b}=4, \mu=10^{-3}\right)$, c) Híbrido $\left(M_{f}=5, M_{b}=M_{r}=4, N=3\right.$, $\left.\mu=10^{-3}, \mu_{\mathrm{RTRL}}=10^{-2}\right), \mu_{\theta}=10^{-2}$; d) Módulos dos zeros do canal $\mathrm{H}_{8}$ ao longo das iterações; $\mathrm{SNR}=25 \mathrm{~dB}, 4-\mathrm{QAM}$.

\subsection{Conclusões}

Neste capítulo, foram propostos um algoritmo concorrente, denominado NDEG-SDD-CMA e um DFE híbrido, formado por dois filtros lineares e uma rede neural recorrente.

O NDEG-SDD-CMA é um algoritmo para adaptação autodidata do DFE e sinalização $M$-QAM. Ele foi inspirado no CMA+SDD e considera a operação do DFE-CMA-FB de forma concorrente com o algoritmo SDD. Através de simulações, verificou-se que esse algoritmo apresenta um comportamento superior ao do DFE-CMA-FB e próximo ao do algoritmo LMS para uma relação sinal-ruído alta.

O DFE híbrido proposto é menos afetado por propagação de erros que o DFE. Utilizando o DFE-CMA-FB na adaptação dos filtros lineares, ele evita soluções degeneradas. Através de simulações, foi mostrado que esse equalizador pode apresentar um desempenho melhor que o DFE ou que equalizadores baseados na RNN. Por exemplo, ele apresentou uma velocidade de convergência maior, um desempenho quase ótimo para um canal não-linear e uma boa capacidade de tracking para um canal variante no tempo. Além disso, seu algo- 
ritmo de treinamento, formado pelo algoritmo RTRL autodidata e o DFE-CMA-FB, possui uma complexidade computacional que possibilita sua implementação. Cabe observar que o treinamento dos filtros lineares do DFE híbrido também pode ser feito com o NDEG-SDDCMA. Neste caso, espera-se uma melhora de desempenho, face ao algoritmo não-concorrente DFE-CMA-FB.

Embora as estruturas consideradas neste capítulo sejam não-lineares, o método da Seção 1.5, também se aplica à obtenção do NDEG-SDD-CMA e à do algoritmo de adaptação do DFE híbrido.

A extensão do NDEG-SDD-CMA e do DFE híbrido para o caso multiusuário vai ser deixada para um trabalho futuro. É relevante notar que o AC-CMA e o SWA também podem ser usados na adaptação do DFE autodidata. Para isso, eles devem ser modificados no sentido de se evitar soluções degeneradas. Uma análise teórica a respeito de como a RNN controla o mecanismo de realimentação de erro também será deixada para um trabalho futuro. 


\section{Capítulo 6}

\section{Conclusões e trabalhos futuros}

Desde o surgimento do algoritmo do Módulo Constante [Godard, 1980; Treichler \& Agee, 1983], a equalização autodidata baseada em estatísticas de ordem superior (HOS) tem sido amplamente estudada. Na literatura, existem diferentes abordagens, destacando-se a proposição de novos algoritmos, análises de convergência, estabilidade e tracking e a utilização de estruturas não-lineares. A natureza não-linear e a multimodalidade dos critérios autodidatas tornam a análise de convergência e estabilidade dos algoritmos relativamente complexa. Quando se consideram aproximações estocásticas, um fator adicional a ser levado em conta é a qualidade das estimativas. As diferentes análises para o algoritmo do Módulo Constante não são conclusivas, principalmente por serem baseadas em considerações não muito realistas (veja, e.g., [Li \& Ding, 1995; Zeng et. al, 1999; Rupp \& Sayed, 2000; Schniter et al., 2001]). Diante disso, o intervalo do passo de adaptação que assegura a convergência do CMA ainda é um problema em aberto.

No presente trabalho, foram propostos seis novos algoritmos autodidatas, um equalizador híbrido e feitas duas análises de convergência e duas de tracking. Os novos algoritmos propostos foram: algoritmo do Gradiente Projetado não-normalizado, AC-CMA, MU-AC-CMA, MU-SWA, NDEG-SDD-CMA e o algoritmo para adaptação autodidata do DFE híbrido. Eles podem apresentar características de convergência, tracking e taxa de erro de símbolo mais interessantes que os existentes na literatura. Com relação ao algoritmo do Gradiente Projetado não-normalizado, o objetivo foi obter um algoritmo mais geral, cuja análise de convergência serviu de inspiração para a análise do SD-CMA, na qual foi obtido o intervalo do passo de 
adaptação que assegura sua convergência. Embora esse algoritmo não tenha facilidade de implementação na prática, sua análise pode abrir novas perspectivas neste cenário. Com relação aos algoritmos do gradiente estocástico que satisfazem a relação de conservação de energia, foram feitas análises de tracking nos casos SISO e MIMO. Embora elas estejam baseadas em suposições não muito realistas, verificou-se por simulações que há uma boa concordância entre o MSE teórico e o experimental.

Se a análise do CMA exige ferramentas matemáticas complexas, a de outros algoritmos autodidatas, como o AC-CMA, DFE-CMA-FB e NDEG-SDD-CMA, se mostram mais difíceis ainda. A análise teórica do DFE híbrido apresenta um entrave adicional que é a natureza não-linear da rede neural recorrente. Neste caso, não existem resultados nem mesmo para a adaptação supervisionada da rede. Por isso, a análise desse esquema e de alguns dos algoritmos propostos nesta tese são deixados para trabalhos futuros.

A tese foi estruturada em seis capítulos, sendo que os capítulos 2, 3 e 5 estão relacionados com a equalização monocanal e o Capítulo 4 com a multicanal. Foram feitas inúmeras simulações, utilizando-se diferentes canais de comunicação, cujos modelos foram escolhidos a fim de mostrar a habilidade de cada técnica considerada. Por exemplo, nas simulações com os algoritmos concorrentes, utilizou-se um canal que causa rotação de fase. No caso do DFE, foram utilizados canais esparsos, não-lineares e variantes no tempo. As principais contribuições estão esquematizadas na Tabela 6.1 e as conclusões apresentadas a seguir.

\section{Algoritmos determinísticos para equalização monocanal}

As funções custo do Módulo Constante (Godard) e de Shalvi-Weinstein foram abordadas, sendo obtidos seus gradientes, pontos estacionários e algoritmos determinísticos. Neste contexto, pôde-se concluir o seguinte:

- os algoritmos do Módulo Constante (SD-CMA) e de Shalvi-Weinstein (SD-SWA) baseados no gradiente determinístico minimizam efetivamente a função custo de Godard, dado que se utilizam as estatísticas do sinal transmitido ao invés das estatísticas do sinal de saída do equalizador;

- o SD-SWA pode ser interpretado como sendo o SD-CMA com um passo ótimo para velocidade de convergência que utiliza a matriz de autocorrelação inversa; 


\begin{tabular}{|c|c|}
\hline \multicolumn{2}{|r|}{ Equalização monocanal } \\
\hline Capítulo & Contribuições \\
\hline $\begin{array}{l}2 \text { - Algoritmos } \\
\text { determinísticos }\end{array}$ & $\begin{array}{l}\text {-Algoritmo do Gradiente Projetado não-normalizado } \\
\text { e sua análise de convergência (Teorema 1) } \\
\text {-Análise de convergência do SD-CMA (Teorema 2) }\end{array}$ \\
\hline $\begin{array}{l}3 \text { - Algoritmos } \\
\text { estocásticos }\end{array}$ & $\begin{array}{l}\text {-AC-CMA } \\
\text {-Análise de tracking (Teorema 3) }\end{array}$ \\
\hline $5-\mathrm{DFE}$ & $\begin{array}{l}\text {-NDEG-SDD-CMA } \\
\text {-DFE híbrido e seu algoritmo de adaptação }\end{array}$ \\
\hline \multicolumn{2}{|r|}{ Equalização multicanal } \\
\hline Capítulo & Contribuições \\
\hline $\begin{array}{l}4 \text { - Algoritmos } \\
\text { estocásticos }\end{array}$ & $\begin{array}{l}\text {-MU-SWA } \\
\text {-MU-AC-CMA } \\
\text {-Análise de tracking (Teorema 4) }\end{array}$ \\
\hline
\end{tabular}

Tabela 6.1: Principais contribuições da tese.

Os algoritmos do tipo Super-Exponencial no domínio da resposta combinada foram revisitados e um algoritmo do Gradiente Projetado não-normalizado proposto. A partir dos resultados de [Mboup \& Regalia, 2000], uma análise de convergência desse algoritmo foi apresentada no Teorema 1. Inspirando-se nesse teorema e reescrevendo o SD-CMA no domínio da resposta combinada, obteve-se o Teorema 2 que estabelece o intervalo do passo de adaptação que assegura a convergência do algoritmo. Neste contexto, as seguintes conclusões puderam ser tiradas:

- o intervalo do passo de adaptação que garante a convergência do algoritmo do Gradiente Projetado, obtido em [Mboup \& Regalia, 2000, Th.1], pode ser interpretado como um caso particular do Teorema 1;

- através de simulações, verificou-se que se o passo de adaptação do SD-CMA for escolhido a cada instante de tempo de acordo com o Teorema 2, sua convergência será assegurada, independente da qualidade das estimativas e da margem de segurança do limite máximo do intervalo; 
- embora esse resultado seja uma contribuição importante na análise de algoritmos autodidatas, ele é válido apenas para o algoritmo que utiliza o gradiente determinístico, estimado de forma exata ou em bloco.

\section{Algoritmos estocásticos para equalização monocanal}

Os algoritmos CMA e SWA foram obtidos a partir do SD-CMA e do SD-SWA, considerando aproximações estocásticas. Além disso, um algoritmo do tipo "acelerador", denominado ACCMA, foi proposto. Comparando esses algoritmos pôde-se concluir que o AC-CMA apresenta:

- uma complexidade computacional maior que a do CMA, mantendo o número de operações proporcional a $M$;

- um desempenho intermediário, se comportando ora próximo do SWA, ora próximo do CMA;

- capacidade de evitar mínimos locais suaves;

- uma boa capacidade de tracking apresentando, em algumas situações, um desempenho próximo ao do SWA e melhor que o do CMA.

Esses algoritmos também foram implementados, utilizando-se a técnica de sobre-amostragem. Neste caso, concluiu-se que:

- dependendo do fator $L$, a diferença de comportamento desses algoritmos deixa de ser significativa;

- o CMA pode apresentar o compromisso mais favorável entre velocidade de convergência e complexidade computacional, desde que o passo de adaptação e o fator de sobreamostragem sejam escolhidos adequadamente;

- o desempenho do SWA está ligado à inicialização da matriz de autocorrelação inversa. Dependendo do valor de $\delta$, ele pode apresentar problemas de instabilidade numérica;

- o AC-CMA é uma boa alternativa, dado que o intervalo do passo de adaptação que garante sua convergência parece ser maior que o do CMA. Além disso, ele não depende da inicialização da matriz de autocorrelação. 
Foi feita uma análise de tracking para algoritmos que satisfazem a relação de conservação de energia. Partindo dessa relação, considerando um ambiente com um pequeno grau de nãoestacionariedade e assumindo verdadeiras as considerações S1-S7, chegou-se ao Teorema 3 que fornece uma expressão teórica para o MSE. Neste contexto, concluiu-se que:

- há uma boa concordância entre o MSE experimental e o teórico;

- a razão obtida entre o MSE mínimo para o SWA e o CMA é a mesma obtida entre os algoritmos RLS e LMS, o que possibilitou uma extensão direta da comparação desses algoritmos com relação ao tracking para o caso autodidata.

\section{Algoritmos estocásticos para equalização multicanal}

Os algoritmos aproximados para equalização autodidata do caso SISO foram estendidos para o caso MIMO. Inspirando-se nas relações entre o CMA e o SWA, foi proposto um algoritmo denotado MU-SWA, que pode ser interpretado como uma extensão do SWA para o caso multiusuário. O algoritmo do tipo "acelerador", AC-CMA, também foi estendido para o caso MIMO, gerando um novo algoritmo para equalização espaço-temporal, denominado MU-AC-CMA. Comparando esses algoritmos, concluiu-se que:

- às custas de uma complexidade computacional proporcional a $M_{u}^{2}$, o MU-SWA apresenta uma velocidade de convergência maior que a do MU-CMA. No entanto, a matriz de autocorrelação pode perder sua positividade, causando problemas de instabilidade numérica;

- o MU-AC-CMA requer um número de operações proporcional a $M_{u}$ e apresenta um desempenho intermediário com relação à velocidade de convergência e capacidade de tracking, tendo um comportamento ora próximo do MU-SWA, ora próximo do MUCMA;

- dada uma condição inicial, o MU-AC-CMA converge para um mesmo patamar de interferência residual, independente do passo de adaptação e com uma velocidade de convergência maior que a do MU-CMA. Além disso, o intervalo do passo de adaptação que garante a convergência desse algoritmo parece ser maior que o do MU-CMA. Esses resultados foram observados através de simulações. 
A análise de tracking feita no caso SISO também foi estendida para o caso MIMO, obtendo-se

o Teorema 4 a partir das considerações M1-M11. Dessa análise, concluiu-se que:

- dentro da região de convergência dos algoritmos, houve uma boa concordância entre o MSE teórico previsto pelo Teorema 4 e o experimental;

- o Teorema 3, obtido no caso SISO, é um caso particular do Teorema 4;

- as conclusões obtidas no caso SISO puderam ser estendidas diretamente para o caso MIMO, mostrando que há situações em que o MU-CMA apresenta uma capacidade de tracking melhor que a do MU-SWA e vice-versa;

- a análise é válida dentro da região de convergência dos algoritmos e para constelações de módulo não-constante.

\section{Sobre o equalizador com realimentação de decisões}

Inspirando-se no CMA+SDD, foi proposto um algoritmo para adaptação autodidata do DFE e sinalização $M$-QAM, denominado NDEG-SDD-CMA. Ele considera a operação do DFECMA-FB de forma concorrente com o algoritmo SDD. Através de simulações, verificou-se que esse algoritmo apresenta:

- capacidade de evitar soluções degeneradas;

- complexidade computacional que possibilita sua implementação;

- um comportamento superior ao do DFE-CMA-FB e próximo ao do algoritmo LMS para uma relação sinal-ruído alta.

Um DFE híbrido formado por dois filtros lineares e uma rede neural recorrente também foi proposto. Esse equalizador apresenta:

- maior capacidade de evitar propagação de erros que o DFE;

- capacidade de evitar soluções degeneradas, quando o DFE-CMA-FB é utilizado na adaptação dos filtros lineares; 
- um desempenho melhor que os equalizadores baseados em rede neural recorrente ou em realimentação de decisões. Por exemplo, ele apresentou uma velocidade de convergência maior, um desempenho quase ótimo para um canal não-linear e uma boa capacidade de tracking para um canal variante no tempo;

- um algoritmo de treinamento, formado pelo algoritmo RTRL autodidata e o DFECMA-FB, com uma complexidade computacional que permite sua implementação.

\section{Trabalhos futuros}

Diante das contribuições e conclusões expostas, existem inúmeras possibilidades de trabalhos futuros. Dentre elas, destacam-se:

TF1 implementação do SWA e do MU-SWA, utilizando-se a decomposição QR para evitar divergência;

TF2 implementação dos algoritmos do caso MIMO, utilizando-se a sobre-amostragem e os algoritmos concorrentes;

TF3 treinamento dos filtros lineares do DFE híbrido com o NDEG-SDD-CMA;

TF4 extensão do NDEG-SDD-CMA e do DFE híbrido para o caso MIMO;

TF5 modificações nos algoritmos AC-CMA e o SWA para que evitem soluções degeneradas no caso da adaptação autodidata do DFE;

TF6 análise de tracking para constelações de módulo constante no caso MIMO;

TF7 extensão do Teorema 2 para o algoritmo do Módulo Constante que utiliza o gradiente estocástico;

TF8 análise de convergência e de tracking para os algoritmos AC-CMA e MU-AC-CMA;

TF9 análise de convergência da RNN e do DFE híbrido.

O TF1 é tema de uma dissertação de mestrado que está em andamento [Bernardes, 2004]. Os TF2-TF5 são trabalhos que envolvem diferentes implementações e simulações, sendo de realização mais simples que os TF6-TF9. Estes são de solução mais difícil, pois exigem ferramentas matemáticas relativamente complexas. 


\section{Referências Bibliográficas}

AMARI, S.; CICHOCKI, A.; YANG, H. H. Blind Signal Separation and Extraction: neural and information-theoretic approaches. In: HAYKIN, S. (Ed.) Unsupervised Adaptive Filtering. New York: John Wiley \& Sons, Inc., 2000, v. I. Chap. 3, p. 63-138.

AMARI, S.; CICHOCKI, A. Adaptive blind signal processing - neural network approaches. Proceedings of the IEEE, v. 86, p. 2026-2048, Oct. 1998.

ASTRÖM, K. J.; WITTENMARK B. Adaptive Control, 2. ed. Massachusetts: AddisonWesley, 1995.

BELL, A. J. Information Theory, Independent-Component-Analysis, and Applications. In: HAYKIN, S. (Ed.) Unsupervised Adaptive Filtering. New York: John Wiley \& Sons, Inc., 2000, v. I. Chap. 6, p. 237-264.

BENVEniste, A.; MÉTIVIER, M.; PRIOURET, P. Adaptive Algorithms and Stochastic Approximations. Paris: Springer-Verlag, 1987.

BENVENISTE, A.; GOURSAT, M.; RUGET, G. Robust identification of a nonminimum phase system: blind adjustment of a linear equalizer in data communications. IEEE Transactions on Automatic Control, v. AC-25, p. 385-399, June 1980.

BERCHER, J. F.; VIGNAT, C. Estimating the entropy of a signal with applications. Transactions on Signal Processing, v. 48, p. 1687-1694, June 2000.

BERNARDES; D. B. Algoritmos robustos para equalização espaço-temporal. 2004. Qualificação (Mestrado em Engenharia Elétrica). Universidade Presbiteriana Mackenzie, São Paulo, 2004. 
BORYS, A. Nonlinear aspects of telecommunications: discrete volterra series and nonlinear echo cancellation. New York: CRC Press, 2001.

BOUCHIRED, S.; ROVIRAS, D.; CASTANI, F. Equalisation of satellite mobile channels with neural network techniques. Space Communications, v. 15, p. 209-220, 1998/1999.

BROSSIER, J. M. Signal et communication numérique: égalisation et synchronisation. Paris: Hermes, 1997.

CARDOSO, J. F. Blind signal separation: statistical principles. Proceedings of the IEEE, v. 86, p. 2009-2025, Oct. 1998.

CARDOSO, J. F. Informax and maximum likelihood for blind source separation. IEEE Signal Processing Letters, v. 4, p. 112-114, Apr. 1997.

CARTWRIGHT, K. V. Blind phase recovery in general QAM communication system using alternative higher order statistics. IEEE Signal Processing Letters, v. 6, p. 327-329, Dec. 1999.

CASAS, R. A. et al. On initialization strategies for blind adaptive DFEs. In: WIRELESS COMMUNiCATIONS AND NETWORKING CONFERENCE, 1999, New Orleans. Proceedings of WCNC'99. v. 2, p. 792-799.

CASAS, R. A. et al. Blind adaptation of decision feedback equalizers based on the constant modulus algorithm. In: ASILOMAR CONFERENCE ON SIGNALS, SYSTEMS \& COMPUTERS, 29., 1995, Pacific Groove. Proceedings. v. 1, p. 698-702.

CASAS, R. A. Blind adaptive decision feedback equalization: a class of bad channels 1996. 53p. Thesis (Master of Science). Cornell University, Ithaca, 1996.

CASTEDO, L.; ESCUDERO, C. J.; DAPENA, A. A blind signal separation method for multiuser communications. IEEE Transactions on Signal Processing, v. 45, p. 1343-1348, May 1997.

CAVALCANTE, C. C. et al. On the use of higher order statistics for blind source separation. In: SIMPÓSIO BRASILEIRO DE TELECOMUNICAÇÕES, 20., 2003, Rio de Janeiro. Anais. 1 CD-ROM. 
CAVAlCANTI, F. R. P.; BRAndÃO, A. L.; ROMAnO, J. M. T. A Generalized Constant Modulus Algorithm. In: GLOBECOM/COMMUNICATIONS THEORY MINICONFERENCE, 1998, Sydney. Proceedings. 1 CD-ROM.

CHEN, L.; KUSAKA, H.; KOMINAMI, M. Blind phase recovery in QAM Communication Systems using Higher Order Statistics. IEEE Signal Processing Letters, v. 3, p. 147-149, May 1996.

CHEN, S.; COOK, T. B.; ANDERSON, L. C. A comparative study of two blind FIR equalizers. Signal Processing, v.14, p. 18-36, 2004a.

CHEN, S.; CHNG, E. S. Concurrent Constant Modulus Algorithm and Soft Decision scheme for fractionally-spaced blind equalization. In: IEEE INTERNATIONAL CONFERENCE ON COMMUNICATIONS, 2004b, Paris. Proceedings of ICC'2004. p. 2342-2346.

CHEN, S. Low complexity concurrent constant modulus algorithm and soft directed scheme for blind equalization. IEE Proceedings - Vision, Image, and Signal Processing, v. 150, p. 312-320, Oct. 2003.

CHEN, S.; MClAUGHLIN, S.; GRANT, P. M.; MUlGREW, B. Multi-stage clustering equaliser. IEEE Transactions on Communications, v. 43, p.701-705, Feb./Mar./Abr. 1995.

CHI C.; WANG, F.; CHIANG, M. Two-step lattice super-exponential algorithm for blind equalization of multi-input multi-output channels. In: VEHICULAR TECHNOLOGY CONFERENCE, 2000, Tokyo. Proceedings. p. 634-639.

COMON, P. Independent Component Analysis: a new concept? Signal Processing, v. 36, p. 287-314, 1994.

COSTA NETO, P. L. O. Estatística. 2. ed. São Paulo: Editora Edgard Blücher Ltda., 2002.

COWAN, C. F. N.; SEMNANI, S. Time-variant equalization using a novel non-linear adaptive structure. International Journal of Adaptive Control and Signal Processing, v.12, p. 195-206, 1998. 
DE CASTRO, F. C. C.; DE CASTRO, M. C. F.; ARANTES, D. S. Concurrent blind deconvolution for channel equalization. In: IEEE INTERNATIONAL CONFERENCE ON COMMUniCATIONS, 2001, Helsink. Proceedings of ICC'2001. v. 2, p. 366-371.

DESTRO FILHO, J. B. Egalisation aveugle de canaux de communication à l'aide d'algorithmes de Bussgang de réseaux neuronaux. 1998. 261p. Thèse (Doctorat en Sciences Pour l’Ingénieur). Université de Nice-Sofhia Antipolis, Nice, 1998.

DING, Z.; LI, Y. Blind Equalization and Identification. New York: Marcel Dekker, 2001.

DINIZ, P. S. R. Nonlinear adaptive filtering. Espoo: Helsinki University of Technology / Laboratory of Telecommunications Technology, 1998. 61p., Report 4/98. ISBN 951-224274-5.

DONOHO, D. On minimum entropy deconvolution. In: FINDLEY, F. D. (Ed.). Applied Time Series Analysis II. New York: Academic, 1981.

ENDRES, T. J. Equalizing with fractionally-spaced constant modulus an second-orderstatistics blind receivers. 1997. 163p. Thesis (Ph.D.). Cornell University, Ithaca, 1997.

ERDOGMUS, D.; PRINCIPE, J. C. An error-entropy minimization for supervised training of nonlinear adaptive systems. IEEE Transactions on Signal Processing, v. 50, p.1780-1786, July 2002.

ESCUDERO, C. J. Algoritmos Adaptativos Ciegos para Igualación y Separación de Señales. 1998. 140p. Tesis (Doctoral). Universidad de La Coruña, La Coruña, 1998.

EWEDA, E. Comparison of RLS, LMS and sign algorithms for tracking randomly timevarying channels. IEEE Transactions on Signal Processing, v. 42, p. 2937-2944, Nov. 1994.

FERTNER, A. Improvement of bit-error-rate in decision feedback equalizer by preventing decision-error propagation. Proceedings of IEEE Transactions on Signal Processing, v. 46, p. 1872-1877, July 1998 .

FORNEY JR., G. D. The Viterbi algorithm. Proceedings of the IEEE, v. 61, p. 268-278, Mar. 1973. 
FORNEY JR., G. D. Maximum-likelihood sequence estimation of digital sequences in the presence of intersymbol interference. IEEE Transactions on Information Theory, v.IT-18, p. 363-378, May 1972.

GARDNER, W. A.; SPOONER, M. The cumulant theory of cyclostationary time-series, part I: foundation. IEEE Transactions on Signal Processing, v. 42, p. 3387-3408, Dec. 1994.

GEORGHIADES, C. N. Blind carrier phase acquisition for QAM constellations. IEEE Transactions on Communications, v. 45, p. 1477-1486, Nov. 1997.

GERKEN, M.; PAIT, F. M.; JOJOA, P. E. An Adaptive algorithm with parameter acceleration. In: IEEE INTERNATIONAL CONFERENCE ON ACOUSTICS, SPEECH, AND SIGNAL PROCESSING, 2000, Istambul. Proceedings of ICASSP'2000. p. 17-20.

GHOSH, M. Blind decision feedback equalization for terrestrial television receivers. Proceedings of the IEEE, v. 86, p. 2070-2081, Oct. 1998.

GODARD, D. N. Self-recovering equalization and carrier tracking in two-dimensional data communication systems. IEEE Transactions on Communications, v. COM-28, p. 18671875, Nov. 1980.

GOMES, J.; BARROSO, V. A multichannel lattice algorithm for real-time blind equalization. In: IEEE INTERNATIONAL CONFERENCE ON ELECTRONICS, CIRCUITS, AND SYSTEMS, 1998, Lisboa. Proceedings. v. 1, p. 445-448.

GOMES, J.; BARROSO, V. Unsupervised learning for channel equalization using a neural network data receiver. In: INTERNATIONAL CONFERENCE ON NEURAL NETWORKS, 1995, Perth. Proceedings of ICNN'1995. v. 1, p. 502-506.

GUIDORIZZI, H. L. Um curso de cálculo. v. 2. Rio de Janeiro: Livros Técnicos e Científicos Editora S.A., 1986.

HEIDARI, A.; NASIRI-KENARI, M. A family of simple blind phase recovery algorithms. In: INTERNATIONAL SYMPOSIUM ON INTELLIGENT SIGNAL PROCESSING AND COMmUniCATION, 2000, Honolulu. Proceedings of ISPACS'2000. v. 1, p. 24-28. 
HAYKIN, S. Adaptive digital communication receivers. IEEE Communications Magazine, p. 106-114, Dec. 2000.

HAYKIN, S. Neural Networks. 2. ed. New Jersey: Prentice Hall, 1999.

HAYKIN, S. Adaptive Filter Theory. 3. ed. New Jersey: Prentice Hall, 1996.

HORN, R. A.; JOHnSON, C. R. Matrix Analysis. New York: Cambrige University Press, 1999.

IBNKAHLA, M. Applications of neural networks to digital communications - a survey. Signal Processing, v. 80, p. 1185-1215, 2000.

JOJOA, P. E. Um algoritmo acelerador de parâmetros. 2003. 192p. Tese (Doutorado em Engenharia Elétrica). Escola Politécnica da Universidade de São Paulo, São Paulo, 2003.

JOJOA, P. E. Análise de um algoritmo adaptativo que ajusta a diferença de segunda ordem dos parâmetros. 1999. Dissertação (Mestrado em Engenharia Elétrica). Escola Politécnica da Universidade de São Paulo, São Paulo, 1999.

JOJOA, P. E.; GERKEN, M.; PAIT, F. M. Sobre o algoritmo acelerador para filtragem adaptativa. In: SIMPÓSIO BRASILEIRO DE TELECOMUNICAÇÕES, 19., 2001a, Fortaleza. 1 CD-ROM.

JOJOA, P. E.; GERKEN, M.; PAIT, F. M. An Adaptive Algorithm with Parameter Acceleration. In: WORKSHOP ON ADAPTATION AND LEARNING CONTROL AND SIGNAL PROCESSING, 2001b, Cernobio-Como. Proceedings of ALCOSP'2001. p. 331335.

KAILATH, T. Linear Systems. New Jersey: Prentice Hall, 1980.

KAILATH, T.; SAYED, A. H.; HASSIBI, B. Linear Estimation. New Jersey: Prentice Hall, 2000.

KARAOGUZ, J.; ADARLAN, S. H. A soft decision-direct blind equalization algorithm applied to equalization of mobile communication channels. In: IEEE INTERNATIONAL 
CONFERENCE ON COMMUNICATIONS, 1992, Chicago. Proceedings of ICC'92. v. 3, p. 343.4.1-343.4.5.

KECHRIOTIS, G.; ZERVAS, E.; MANOLAKOS, E. S. Using recurrent neural networks for blind equalization of linear and nonlinear communication channels. In: MILITARY COMmuniCATIOnS CONFEREnCE, 1992, San Diego. Proceedings of MILCOM'92. p. 784-788.

KECHRIOTIS, G.; ZERVAS, E.; MANOLAKOS, E. S. Using recurrent neural networks for adaptive communication channel equalization. IEEE Transactions on Neural Networks, v. 5, p. 267-278, Mar. 1994a.

KECHRIOTIS, G.; MANOLAKOS, E. S. Training fully recurrent neural networks with complex weights. IEEE Transactions on Circuits and Systems-II: Analog and Digital Signal Processing, v. 41, n. 3, Mar. 1994b.

KOFIDIS, E. Blind Source Separation: fundamentals and recent advances. In: SIMPÓSIO BRASILEIRO DE TELECOMUNICAÇÕES, 19., 2001, Fortaleza. Minicurso.

LABAT, J.; MACCHI, O.; LAOUT, C. Adaptive decision feedback equalization: can you skip the training period? IEEE Transactions on Communications, v. 46, p. 921-930, July 1998.

LAMBERT, R.; NIKIAS, C. Blind deconvolution of multipath mixtures. In: HAYKIN, S. (Ed.) Unsupervised Adaptive Filtering. New York: Jonh Wiley \& Sons, 2000. v. 1.

LATHI, B. P. Modern Digital and Analog Communication Systems. 3. ed. New York: Oxford University Press, 1998.

LEBLANC, J. P. Effects of source distributions and correlation on fractionally spaced blind constant modulus algorithm equalizers. 1995. 184p. Thesis (Ph.D.). Cornell University, Ithaca, 1995.

LI, Y; DING, Z. Global convergence of fractionally spaced Godard (CMA) adaptive equalizers. IEEE Transactions on Signal Processing, v. 44, p. 818-826, Apr. 1996. 
LI, Y.; DING, Z. Convergence analysis of finite blind adaptive equalizers. IEEE Transacitons on Signal Processing, v. 43, p. 2120-2129, Sept. 1995.

LIAVAS, A. P.; REGALIA, P. A.; DELMAS, J. P. Robustness of least-squares and subspace methods for blind channel identification/equalization with respect to effective channel undermodeling/overmodeling. IEEE Transactions on Signal Processing, v.48, p. 1477-1481, May 2000.

LICCIARDI JR., A. N. Sobre o método super-exponencial de deconvolução autodidata. 2003. Dissertação (Mestrado em Engenharia Elétrica). Universidade Presbiteriana Mackenzie, São Paulo, 2003.

LUENBERGER, D. G. Linear and Nonlinear Programming. 2. ed. Massachusetts: AddisonWesley, 1984.

LUO, Y.; CHAMBERS, J. A. Steady-state mean-square error analysis of the cross-correlation and constant modulus algorithm in a MIMO convolutive system. IEE Proceedings - Vision, Image, and Signal Processing, v. 149, p. 196-203, Aug. 2002.

LUO, Y.; CHAMBERS, J. A. Quasi-Newton constant modulus adaptive algorithm for use in multi-user communication systems. Electronic Letters, v. 37, p. 264-265, Feb. 2001.

LUO, Y.; CHAMBERS, J. A. Quasi-Newton cross correlation and constant modulus adaptive algorithm for space-time blind equalization. In: INTERNATIONAL CONFERENCE ON MATHEMATHICS IN SIGNAL PROCESSING, Warwick, 2000. Proceedings.

MACCHI, O.; EWEDA, E. Convergence analysis of self-adaptive equalizers. IEEE Transactions on Information Theory, v. IT-3, p. 161-176, 1984.

MAGEE JR., F. R.; PROAKIS, J. G. Adaptive maximum-likelihood sequence estimation for digital signaling in the presence of intersymbol interference. IEEE Transaction on Information Theory, p. 120-124, Jan. 1973.

MAI, J.; SAYED, A. H. A feedback approach to the steady-state performance of fractionally spaced blind adaptive equalizers. IEEE Transactions on Signal Processing, v. 48, p. 80-91, Jan. 2000. 
MARPLE JR., S. L. Digital Spectral Analysis with applications. New Jersey: Prentice Hall, 1987.

MARTONE, M. A blind adaptive QR-based lattice multi-channel filter for cellular basestation TDMA transceivers with antenna arrays. In: IEEE INTERNATIONAL CONFERENCE ON COMMUniCATIONS, 1997, Montreal. Proceedings of ICC'9\%. p. 147-151.

MBOUP, M.; REGALIA, P. A. A gradient search interpretation of the super-exponential algorithm. IEEE Transactions on Information Theory, v. 46, p. 2731-2734, Nov. 2000.

MENDEL, J. M. Tutorial on higher-order statistics (spectra) in signal processing and system theory: theoretical results and some applications. Proceedings of the IEEE, v. 79, Mar. 1991.

MÍGUEZ, J.; ESCUDERO, C. J.; CASTEDO, L. Blind adaptive interference suppression for direct-sequence CDMA. In: EUROPEAN WORKSHOP ON INFORMATION SCIENCES AND SYSTEMS, 1997, Toulouse. 1 CD-ROM.

MIRANDA, M. D. Desenvolvimento de algoritmos RLS aproximados para equalização autodidata. São Paulo: FAPESP, 1999. (Relatório Final de Pós-Doutorado).

MOUlineS, E.; DUhAMEL, P.; CARDOSO, J. F.; MAYRARGUE, S. Subspace methods for the blind identification of multichannel FIR filters. IEEE Transactions on Signal Processing, v. 43, p. 516-525, Feb. 1995.

MULGREW, B. Applying radial basis function. IEEE Signal Processing Magazine, v. 13, p. 50-65, Mar. 1996.

OH, S. K.; STAPLETON, S. P. Blind phase recovery using finite alphabet properties in digital communications. Electronic Letters, v. 33, p. 175-176, Jan. 1997.

PAIT, F. M. A Tuner that Accelerates Parameters. Systems $\&$ Control Letters, v. 35 p. 65-68, 1998.

PAPADIAS, C. B. Blind Source Separation based on a multiuser kurtosis maximization criterion. IEEE Transactions on Signal Processing, v. 48, p. 3508-3519, Dec. 2000a. 
PAPADIAS, C. B. Blind separation of independent sources based on multiuser kurtosis optimization criteria. In: Haykin, S. (Ed.) Unsupervised Adaptive Filtering. New York: John Wile \& Sons, 2000b. v. 2, p. 147-179.

PAPADIAS, C. B.; SLOCK, D. T. M. Normalized sliding window constant modulus and decision-direct algorithms: a link between blind equalization and classical adaptive filtering. IEEE Transactions on Signal Processing, v. 45, p. 231-235, Jan 1997.

PAPADIAS, C. B.; PAULRAJ, A. J. A constant modulus algorithm for multiuser signal separation in presence of delay spread using antenna arrays. IEEE Signal Processing Letters, v. 4, p. 178-181, June 1997a.

PAPADIAS, C. B.; PAULRAJ, A. On the blind separability of multiple user signals in the presence of delay spread. In: ASILOMAR CONFERENCE ON SIGNALS, SYSTEMS \& COMPUTERS, 31., 1997b. Proceedings. v. 2, p. 1400-1404.

PAPADIAS, C. B.; PAULRAJ, A. Decision-feedback equalization and identification of linear channels using blind algorithms of the bussgang type. In: ASILOMAR CONFERENCE ON SIGNALS, SYSTEMS \& COMPUTERS, 29., 1995, Pacific Groove. Proceedings. v. 1, p. 335-340.

PAPOULIS A. Probability, Random Variables and Stochastic Processes. 2. ed. New York: McGraw-Hill, Inc., 1984.

PAULRAJ, A. J.; PAPADIAS, C. B. Space-time processing for wireless communications. IEEE Signal Processing Magazine, p. 49-83, Nov. 1997.

PEARLMUTTER, B. A. Gradient calculations for dynamic recurrent neural networks: a survey. IEEE Transactions on Neural Networks, v. 6, p. 1212-1228, Sept. 1995.

PICINBONO, B. Random Signals and Systems. New Jersey: Prentice Hall, Inc., 1993.

PORAT, B. Digital processing of random signals: theory and methods. New Jersey: Prentice Hall, Inc., 1994.

PRESS, W. H. et al. Numerical Recipes: the art of scientific computing. New York: Cambridge University Press, 1986 
PROAKIS, J. G. Digital Communications. 3. ed. New York: McGraw-Hill, 1996.

QURESHI, S. H. Adaptive equalization. Proceedings of the IEEE, v.73, p. 1349-1387, Sept. 1985.

REGALIA, P. A. Egalisation Auto-Didacte. Evry: Institute National des Télécommunications, 1998. (Publication interne).

REGALIA, P. A. On the equivalence between the Godard and Shalvi-Weinstein schemes of blind equalization Signal Processing, v. 73, p. 185-190, 1999.

REGALIA, P. A; MBOUP, Properties of some blind equalization criteria in noisy multiuser environments. IEEE Transactions on Signal Processing, v. 49, p. 3112- 3121, Dec. 2001.

REGALIA, P. A; MBOUP, M. Undermodeled equalization: a characterization of stationary points for a family of blind criteria. IEEE Transactions on Signal Processing, v. 47, p. 760-770, Mar. 1999.

REUTER, M. et al. Mitigating error propagation effects in a decision feedback equalizer. IEEE Transactions on Communications, v. 49, p.2028-2041, Nov. 2001.

RONTOGIANNIS, A. A.; BERBERIDIS, K. Efficient Decision Feedback Equalization for Sparse Wireless Channels. IEEE Transactions on Wireless Communications, v. 2, p. 570581, May 2003.

ROY, P. Fractionally Spaced Blind Equalizer Performance Improvement. 2000. 100p. Thesis (Master of Science in Electrical Engineering). Virginia Polytechnic Institute and State University, Blacksburg, 2000.

RUPP, M.; SAYED, A. H. A time-domain feedback analysis of filtered-error adaptive gradient algorithms. IEEE Transactions on Signal Processing, v. 44, p. 1428-1439, June 1996.

RUPP, M.; SAYED, A. H. On the convergence of blind adaptive equalizers for constantmodulus signals. IEEE Trans. on Communications, v. 48, p. 795-803, May 2000.

SANTAMARÍA, I. et al. A fast algorithm for adaptive blind equalization using order$\alpha$ Renyi's entropy. In: IEEE INTERNATIONAL CONFERENCE ON ACOUSTICS, 
SPEECH, AND SIGNAL PROCESSING, 2002a, Orlando. Proceedings of ICASSP'2002. p. 2657-2660.

SANTAMARÍA, I.; ERDOGMUS, D.; PRINCIPE, J. C. Entropy minimization for supervised digital communications channel equalization. IEEE Transactions on Signal Processing, v. 50, p. 1184-1192, May 2002b.

SATO, Y. A method of self-recovering equalization for multi-level amplitude modulation systems. IEEE Transactions on Communications, v. COM-23, p. 679-682, 1975.

SAYED, A. H. Fundamentals of Adaptive Filtering. 1. ed. New Jersey: John Wiley \& Sons, 2003.

SCHIRTZINGER, T. A.; JENKINS, W. K. Designing adaptive equalizers based on the constant modulus error criterion. In: IEEE INTERNATIONAL SYMPOSIUM ON CIRCUITS AND SYSTEMS, 1995, Seattle. Proceedings of ISCAS'95. v. 2, p. 1094-1097.

SCHNITER, P. et al. Performance analysis of godard-based blind channel identification. IEEE Transactions on Signal Processing, v. 49, p. 1757-1767, Aug. 2001.

SHALVI, O.; WEINSTEIN E. Universal Methods for blind deconvolution. In: HAYKIN, S. (Ed.) Blind Deconvolution. New Jersey: Prentice Hall, 1994. Cap. 4.

SHALVI, O.; WEINSTEIN, E. Super-exponential methods for blind deconvolution. IEEE Transactions on Information Theory, v. 39, p. 504-519, 1993.

SHALVI, O.; WEINSTEIN, E. New criteria for blind deconvolution of non-minimum phase systems (channels). IEEE Transactions on Information Theory, v. IT-36, p. 312-321, 1990.

SHANNON, C. E. A mathematical theory of communication. Bell System Technical Journal, v. 27, p. 379-423, 623-656, July/Oct. 1948.

SHIMAMURA, T.; COWAN, C. F. N. Equalisation of time variant multipath channels using amplitude banded techniques. In: IEEE INTERNATIONAL CONFERENCE ON ACOUSTICS, SPEECH, AND SIGNAL PROCESSING, 1997, Munich. Proceedings of ICASSP'97. p. 2497-2500. 
SIGNAL PROCESSING SOCIETY and NATIONAL SCIENCE FOUNDATION. The Signal Processing Information Base (SPIB). Arquivos de dados (chan4.mat, chan9.mat). Disponível em: <http://spib.rice.edu/spib/microwave.html>. Acesso em 14 fev. 2003.

SILVA, M. T. M.; MIRANDA, M. D. Tracking issues of some blind equalization algorithms. IEEE Signal Processing Letters, v. 11, p. 760-763, Sept. 2004a.

SILVA, M. T. M.; GERKEN, M.; MIRANDA, M. D. A Blind Hybrid Decision Feedback Equalizer. In: SIMPÓSIO BRASILEIRO DE REDES NEURAIS, 8., 2004b, São Luís. Anais. 1 CD-ROM.

SILVA, M. T. M.; MIRANDA, M. D.; SOARES, R. Concurrent Blind Decision Feedback Equalizer. In: INTERNATIONAL WORKSHOP ON TELECOMMUNICATIONS, 2004c, Santa Rita do Sapucaí. Proceedings of IWT'2004, p. 107-112.

SILVA, M. T. M.; GERKEN, M.; MIRANDA, M. D. An accelerated constant modulus algorithm for space-time blind equalization. In: EUROPEAN SIGNAL PROCESSING CONFERENCE, 12., 2004d, Vienna. Proceedings of EUSIPCO’2004. p. 1853-1856.

SILVA, M. T. M.; MIRANDA, M. D.; LICCIARDI JR., A. N. A robust algorithm for blind space-time equalization. In: IEEE INTERNATIONAL CONFERENCE ON ACOUSTICS, SPEECH AND SIGNAL PROCESSING, 2004e, Montreal. Proceedings of ICASSP'2004. v. IV, p. 857-860.

SILVA, M. T. M.; GERKEN, M.; MIRANDA, M. D. Um algoritmo do Módulo Constante Acelerado para equalização espaço-temporal. In: SIMPÓSIO BRASILEIRO DE TELECOMUNICAÇÕES, 20., 2003, Rio de Janeiro. Anais. 1 CD-ROM.

SILVA, M. T. M.; GERKEN, M. A RNN-LC Hybrid Equalizer. In: EUROPEAN SIGNAL PROCESSING CONFERENCE, 11., 2002a, Toulouse. Proceedings of EUSIPCO'2002. p. 341-344.

SILVA, M. T. M.; GERKEN, M.; MIRANDA, M. D. An Accelerated Constant Modulus Algorithm. In: INTERNATIONAL TELECOMMUNICATIONS SYMPOSIUM, 2002b, Natal. Proceedings of ITS'2002. 1 CD-ROM. 
SILVA, M. T. M. Equalização não-linear de canais de comunicação. 2001. 177p. Dissertação (Mestrado em Engenharia Elétrica). Escola Politécnica da Universidade de São Paulo, São Paulo, 2001.

SILVA, M. T. M.; GERKEN, M. Um equalizador híbrido RNN/LTE. In: SIMPÓSIO BRASILEIRO DE TELECOMUNICAÇÕES, 19., 2001, Fortaleza. Anais. 1 CD-ROM.

SILVA, M. T. M.; GERKEN, M. Um algoritmo acelerador para treinamento de redes neuronais do tipo MLP. In: SIMPÓSIO BRASILEIRO DE TELECOMUNICAÇÕES, 18., 2000a, Gramado. Anais. 1 CD-ROM.

SILVA, M. T. M.; GERKEN, M.; MIRANDA, M. D. Estruturas não-lineares aplicadas à recuperação de sinais binários In: SIMPÓSIO BRASILEIRO DE TELECOMUNICAÇÕES, 18., 2000b, Gramado. Anais. 1 CD-ROM.

SIMMONS, G. F. Cálculo com geometria analítica. v. 2. São Paulo: McGraw-Hill, 1987.

SLOCK, D. T. M. Blind fractionally-spaced equalization, perfect reconstruction filterbanks, and multilinear prediction. In: IEEE INTERNATIONAL CONFERENCE ON ACOUSTICS, SPEECH, AND SIGNAL PROCESSING, 1994, Adelaide. Proceedings of ICASSP'94. p. 585-588.

SONI, R. A.; SCHIRTZINGER ,T. A.; JENKINS, W. K. Learning strategies for adaptive equalizers using the constant modulus error criterion. International Journal of adaptive control and signal processing, v. 12, p. 97-116, Mar. 1998.

STEWART, G. W. Introduction to matrix computations. New York: Academic Press, Inc., 1973.

SZCZECINSKI, L. L.; GEI, A. On the convergence of blind over-sampled decision feedback equalizers. In: DIGITAL SIGNAL PROCESSING WORKSHOP, 9., 2000, Hunt Proceedings of DSP'2000. 1 CD-ROM.

SZCZECINSKI, L. L.; GEI, A. Blind decision feedback equalisers, how to avoid degenerative solutions. Signal Processing, v. 82, p. 1675-1693, 2002. 
TONG, L.; PERREAU, S. Multichannel blind identification: from subspace to maximum likelihood methods. Proceedings of the IEEE, v. 86, p. 1951-1968.

TONG, L.; LIU, D. Blind predictive decision-feedback equalization via the constant modulus algorithm. In: IEEE INTERNATIONAL CONFERENCE ON ACOUSTICS, SPEECH, AND SIGNAL PROCESSING, 1997, Munich. Proceedings of ICASSP'97. p. 3901-3904.

TONG, L.; XU, G.; KAILATH, T. Blind identification and equalization based on secondorder statistics: a time domain approach. IEEE Transactions on Information Theory, v. 41, p. 340-349, Mar. 1994.

TREICHLER, J.; AGEE, B. A new approach to multipath correction of constant modulus signals. IEEE Transactions on Acoustics Speech and Signal Processing, v. ASSP-28, p. 334-358, 1983.

TREICHLER, J. R.; FIJALKOW, I.; JOHNSON JR., C. R. Fractionally spaced equalizers. IEEE Signal Processing Magazine, p. 65-81, May 1996.

UNGERBOECK, G. Adaptive maximum-likelihood receiver for carrier-modulated datatransmission systems. IEEE Transactions on Communications, p. 1128-1137, May 1974.

VAIDYANATHAN, P. P. Multirate systems and filter banks. New Jersey: Englewood Cliffs, 1993.

VARY, P.; WACKERSREUTHER, G. A unified approach to digital polyphase filter banks. $A E \ddot{U}, \mathrm{v}$. Band 37, p.29-34, 1983.

VITERBI, A. J. Error bounds for convolutional codes and asymptotically optimum decoding algorithm. IEEE Transactions on Information Theory, v. IT-13, p. 260-269, Apr. 1967.

WIGGINS, R. A. Minimum entropy deconvolution. Geoexplor., v. 16, p. 21-35, 1978.

XU, G.; LIU, H.; TONG, L.; KAILATH, T. A least-squares approach to blind channel identification. IEEE Transactions on Signal Processing, v. 43, p. 2982-2993, Dec. 1995.

YAN, G.; FAN, H. A Newton-like algorithm for complex variables with application in blind equalization. IEEE Transactions on Signal Processing, v. 48, p. 553-556, Feb. 2000. 
YEUNG, K. L.; YAU, S. F. A cumulant-based super-exponential algorithm for blind deconvolution of multi-input multi-output systems. Signal Processing, p. 141-162, 1998.

YOUSEF, N. R.; SAYED, A. H. A unified approach to the steady-state and tracking analyses of adaptive filters. IEEE Transactions on Signal Processing, v. 49, p. 314-324, Feb. 2001.

YOUSEF, N. R.; SAYED, A. H. A feedback analysis of the tracking performance of blind adaptive equalization algorithms. In: IEEE CONFERENCE ON DECISION AND CONTROL, 1999, Phoenix. Proceedings. v. 1, p. 174-179.

ZENG, H. H.; TONG, L.; JOHNSON JR., R. An analysis of constant modulus receivers. IEEE Transactions on Signal Processing, v. 47, Nov 1999.

ZENG, H. H.; TONG, L.; JOHNSON JR., R. Relationships between the constant modulus and wiener receivers. IEEE Transactions on Information Theory, v. 44, p. 1523-1538, July 1998.

ZISPER, D.; WILLIAMS, R. J. Gradient-based learning algorithm for recurrent networks. In: CHAUVIN, Y.; RUMELHART, D. E. (Eds.) Back-propagation: Theory, Architectures, and Applications. New Jersey: Erlbaum Publ., 1991. 


\section{Apêndice A}

\section{Equalizadores sobre-amostrados}

Um diagrama de blocos de um sistema de comunicação com equalizador sobre-amostrado é apresentado na Figura A.1 [LeBlanc, 1995].

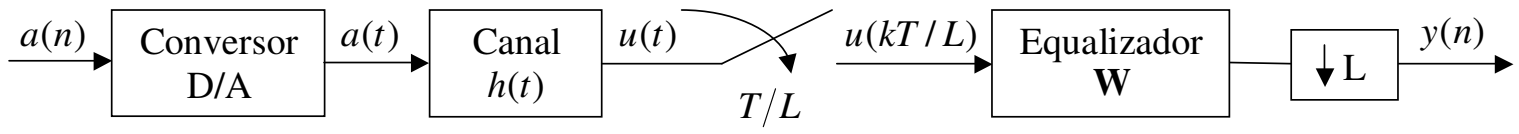

Figura A.1: Diagrama de blocos de um sistema de comunicação com equalizador sobre-amostrado por um fator $L$.

Os símbolos $a(n)$ são transmitidos a cada $T$ unidades de tempo e convertidos por um D/A em um sinal de tempo contínuo dado por

$$
a(t)=\sum_{n=-\infty}^{+\infty} \delta(t-n T) a(n)
$$

Esse sinal passa através de um canal físico, cujo modelo de tempo contínuo $h(t)$ inclui o formatador de pulsos. O sinal de saída do canal antes da amostragem é determinado pela seguinte integral de convolução [LeBlanc, 1995; Treichler et al., 1996; Endres, 1997]

$$
\begin{aligned}
u(t) & =\int_{-\infty}^{+\infty} a(\tau) h(t-\tau) d \tau \\
& =\int_{-\infty}^{+\infty} h(t-\tau)\left[\sum_{n=-\infty}^{+\infty} \delta(\tau-n T) a(n)\right] d \tau \\
& =\sum_{n=-\infty}^{+\infty} a(n) h(t-n T) .
\end{aligned}
$$


Amostrando o sinal recebido a cada $T / L$ unidades de tempo e agrupando as $L$ amostras do intervalo $k T \leq t<(k+1) T$ no vetor $\breve{\mathbf{u}}(k)$, obtém-se

$$
\breve{\mathbf{u}}(k)=\left[\begin{array}{c}
u(k T) \\
u\left(\left(k+\frac{1}{L}\right) T\right) \\
u\left(\left(k+\frac{2}{L}\right) T\right) \\
\vdots \\
u\left(\left(k+\frac{L-1}{L}\right) T\right)
\end{array}\right]=\left[\begin{array}{c}
\sum_{n} a(n) h((k-n) T) \\
\sum_{n} a(n) h\left((k-n) T+\frac{T}{L}\right) \\
\sum_{n} a(n) h\left((k-n) T+\frac{2 T}{L}\right) \\
\vdots \\
\sum_{n} a(n) h\left((k-n) T+\frac{(L-1) T}{L}\right)
\end{array}\right] .
$$

Cabe observar que as seqüências $u\left(\left(k+\frac{i}{L}\right) T\right), i=0, \ldots, L-1$ são saídas de um banco de filtros, cujos subcanais são definidos como

$$
c_{i}(k-n) \triangleq h\left((k-n) T+\frac{i T}{L}\right), \quad i=0,1,2, \ldots, L-1
$$

Utilizando essa definição, o vetor $\breve{\mathbf{u}}(k)$ pode ser reescrito como

$$
\breve{\mathbf{u}}(k)=\left[\begin{array}{c}
u_{0}(k) \\
u_{1}(k) \\
u_{2}(k) \\
\vdots \\
u_{L-1}(k)
\end{array}\right]=\left[\begin{array}{c}
\sum_{n} a(n) c_{0}(k-n) \\
\sum_{n} a(n) c_{1}(k-n) \\
\sum_{n} a(n) c_{2}(k-n) \\
\vdots \\
\sum_{n} a(n) c_{L-1}(k-n)
\end{array}\right] .
$$

Define-se o modelo discreto do canal como

$$
H(z)=h_{0}+h_{1} z^{-1}+\ldots+h_{N_{h}-1} z^{-N_{h}+1}
$$

sendo $\left\{h_{0}, h_{1}, \cdots, h_{N_{h}-1}\right\}$ as amostras obtidas a partir da amostragem de $h(t)$ a cada $T / L$ unidades de tempo. Conseqüentemente, os subcanais de (A.4) são obtidos a partir da decomposição polifásica de $H(z)$ [Vaidyanathan, 1993] e podem ser escritos no domínio da transformada-Z como

$$
C_{i}(z)=h_{i}+h_{i+L} z^{-1}+h_{i+2 L} z^{-2} \ldots, \quad \operatorname{com} i=0,1, \ldots, L-1 .
$$

Essa formulação sugere o modelo multicanal da Figura A.2 [Endres, 1997]. 




Figura A.2: Modelo do canal considerando a decomposição polifásica por um fator $L$.

A saída do equalizador, que deve estar na taxa de símbolos $1 / T$, pode ser escrita como

$$
y(n)=\mathbf{u}^{T}(n) \mathbf{w}
$$

sendo

$$
\mathbf{u}(n)=\left[\begin{array}{llll}
\breve{\mathbf{u}}^{T}(n) & \breve{\mathbf{u}}^{T}(n-1) & \cdots & \breve{\mathbf{u}}^{T}\left(n-M_{c}+1\right)
\end{array}\right]^{T}
$$

o vetor regressor de entrada,

$$
\mathbf{w}=\left[\begin{array}{llll}
\breve{\mathbf{w}}_{0}^{T} & \breve{\mathbf{w}}_{1}^{T} & \cdots & \breve{\mathbf{w}}_{M_{c}-1}^{T}
\end{array}\right]^{T}
$$

o vetor de coeficientes do equalizador e

$$
\breve{\mathbf{w}}_{m}=\left[\begin{array}{llll}
w_{m 0} & w_{m 1} & \cdots & w_{m(L-1)}
\end{array}\right]^{T},
$$

com $m=0,1,2, \ldots, M_{c}-1$. Cabe observar que a dimensão do vetor de coeficientes w é $L M_{c}$. Para facilitar a notação, os coeficientes do equalizador foram considerados fixos. Definem-se ainda os vetores de coeficientes entrelaçados

$$
\mathbf{w}_{i}=\left[\begin{array}{llll}
w_{0 i} & w_{1 i} & \cdots & w_{\left(M_{c}-1\right) i}
\end{array}\right]^{T},
$$

com $i=0,1,2, \ldots, L-1$, que podem ser escritos no domínio da transformada-Z como

$$
W_{i}(z)=w_{0 i}+w_{1 i} z^{-1}+\cdots+w_{\left(M_{c}-1\right) i} z^{-M_{c}+1} .
$$

Essas definições sugerem o equalizador MISO esquematizado na Figura A.3 [Endres, 1997]. 


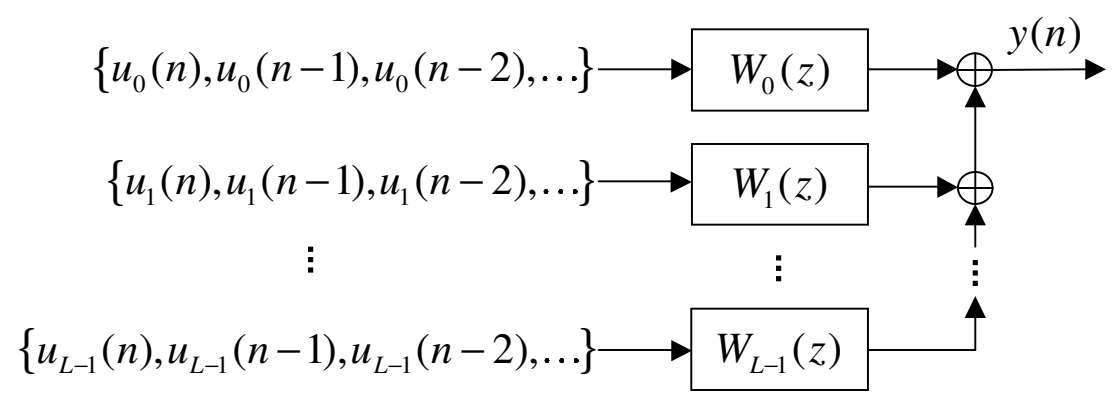

Figura A.3: Modelo do equalizador resultante da decomposição polifásica por um fator $L$.

Combinando as figuras A.2 e A.3 obtém-se a Figura 1.6 da página 12, que caracteriza um sistema de comunicação na ausência de ruído com canal e equalizador sobre-amostrados, sendo equivalente ao diagrama de blocos da Figura A.1 [Treichler et al., 1996; Endres, 1997]. Quando a sobre-amostragem não é considerada, ou seja, quando o equalizador opera na taxa de símbolos $1 / T$, o modelo de tempo discreto do canal se reduz a $C_{0}(z)$ e o equalizador é dado por $W_{0}(z)$. Observando a Figura 1.6 pode-se verificar ainda que há uma certa relação entre o sistema sobre-amostrado e um sistema com diversidade espacial [LeBlanc, 1995]. De fato, a Figura 1.6 também pode ser utilizada para esquematizar um sistema com um usuário e $L$ sensores (antenas) no receptor. Neste caso, os modelos de tempo discreto dos subcanais são caracterizados pelos diferentes percursos e não a partir da amostragem de um mesmo canal $h(t)$.

Por fim, cabe observar que todo algoritmo de equalização autodidata que opera na taxa de símbolos pode ser estendido para o caso de sobre-amostragem [Regalia, 1998]. Para isso, a adaptação dos $L$ subfiltros do equalizador deve ser feita em paralelo considerando um único vetor de coeficientes com dimensão $L M_{c}$ como definido em (A.10). 


\section{Apêndice B}

\section{Cálculos de gradientes}

\section{B.1 Derivada em relação à um vetor complexo}

Sejam $w_{k_{R}}$ e $w_{k_{I}}$ as partes real e imaginária do $k$-ésimo elemento do vetor complexo $\mathbf{w}$, isto é,

$$
w_{k}=w_{k R}+j w_{k I}
$$

A noção de derivada deve estar de acordo com o conceito de diferencial. Em particular, quando se considera mudanças de variável, a regra da cadeia deve ser obedecida [Haykin, 1996]. Desta forma, as derivadas complexas podem ser definidas em termos de derivadas reais, ou seja,

$$
\frac{\partial}{\partial w_{k}}=\frac{1}{2}\left(\frac{\partial}{\partial w_{k R}}-j \frac{\partial}{\partial w_{k I}}\right)
$$

e

$$
\frac{\partial}{\partial w_{k}^{*}}=\frac{1}{2}\left(\frac{\partial}{\partial w_{k R}}+j \frac{\partial}{\partial w_{k I}}\right) .
$$

Essas derivadas satisfazem

$$
\frac{\partial w_{k}}{\partial w_{k}}=1
$$

e

$$
\frac{\partial w_{k}}{\partial w_{k}^{*}}=\frac{\partial w_{k}^{*}}{\partial w_{k}}=0
$$


Com essas definições, a derivada em relação ao vetor complexo $\mathbf{w}=\left[\begin{array}{lllll}w_{0} & w_{1} & \cdots & w_{M-1}\end{array}\right]^{T}$ pode ser escrita como

$$
\frac{\partial}{\partial \mathbf{w}}=\frac{1}{2}\left[\begin{array}{c}
\frac{\partial}{\partial w_{0 R}}-j \frac{\partial}{\partial w_{0 I}} \\
\frac{\partial}{\partial w_{1 R}}-j \frac{\partial}{\partial w_{1 I}} \\
\vdots \\
\frac{\partial}{\partial w_{M-1 R}}-j \frac{\partial}{\partial w_{M-1 I}}
\end{array}\right]
$$

$\mathrm{e}$

$$
\frac{\partial}{\partial \mathbf{w}^{*}}=\frac{1}{2}\left[\begin{array}{c}
\frac{\partial}{\partial w_{0 R}}+j \frac{\partial}{\partial w_{0 I}} \\
\frac{\partial}{\partial w_{1 R}}+j \frac{\partial}{\partial w_{1 I}} \\
\vdots \\
\frac{\partial}{\partial w_{M-1 R}}+j \frac{\partial}{\partial w_{M-1 I}}
\end{array}\right] .
$$

Seja $J(\mathbf{w})$ uma função custo real que define a superfície de erro de um filtro linear transversal. O vetor gradiente e a matriz hessiana dessa superfície de erro são definidos respectivamente como [Haykin, 1996; Kailath et al., 2000]

$$
\nabla_{\mathbf{w}} J(\mathbf{w}) \triangleq \frac{\partial J(\mathbf{w})}{\partial \mathbf{w}^{*}}
$$

e

$$
\mathbf{F}(\mathbf{w}) \triangleq \frac{\partial^{2} J(\mathbf{w})}{\partial \mathbf{w}^{*} \mathbf{w}}=\left[\frac{\partial^{2} J(\mathbf{w})}{\partial w_{k}^{*} w_{l}}\right]_{k, l=0, \ldots, M-1} .
$$

Com base nessas definições e adotando a notação da Seção 1.3, página 7, alguns resultados úteis para o cálculo dos gradientes das funções custo de Godard e de Shalvi-Weinstein são mostrados a seguir.

$$
\begin{aligned}
& \frac{\partial y(n)}{\partial \mathbf{w}^{*}}=\mathbf{0}, \\
& \frac{\partial y^{*}(n)}{\partial \mathbf{w}^{*}}=\mathbf{u}^{*}(n), \\
& \frac{\partial|y(n)|^{p}}{\partial \mathbf{w}^{*}}=(\beta-1) \frac{p}{2}|y(n)|^{p-2} y(n) \mathbf{u}^{*}(n), \\
& \frac{\partial \mathrm{E}\left\{|y(n)|^{2}\right\}^{2}}{\partial \mathbf{w}^{*}}=2(\beta-1) \mathrm{E}\left\{|y(n)|^{2}\right\} \mathrm{E}\left\{y(n) \mathbf{u}^{*}(n)\right\},
\end{aligned}
$$


sendo $\beta=2(=3)$ para o caso complexo (real).

Considerando a definição de $C_{2,2}^{y}$ da Equação (2.20, página 39) e os resultados de (B.10) e (B.11), obtém-se

$$
\frac{\partial C_{2,2}^{y}}{\partial \mathbf{w}^{*}}=2(\beta-1)\left[\mathrm{E}\left\{|y(n)|^{2} y(n) \mathbf{u}^{*}\right\}-\beta \mathrm{E}\left\{|y(n)|^{2}\right\} \mathrm{E}\left\{y(n) \mathbf{u}^{*}\right\}\right]
$$

A partir desses resultados, os vetores gradientes das funções custo de Godard e de ShalviWeinstein são calculados a seguir.

\section{B.2 Função custo de Godard}

Utilizando a regra da cadeia, o vetor gradiente da função custo de Godard da Equação (2.27, página 40), é calculado como

$$
\nabla_{\mathbf{w}} J_{G}(n)=\frac{\partial J_{G}}{\partial \mathbf{w}^{*}}=\mathrm{E}\left\{2\left(|y(n)|^{2}-R_{2}^{a}\right) \frac{\partial|y(n)|^{2}}{\partial \mathbf{w}^{*}}\right\} .
$$

Utilizando (B.10), esse gradiente esse reduz a

$$
\nabla_{\mathbf{w}} J_{G}(n)=2(\beta-1) \mathrm{E}\left\{\left(|y(n)|^{2}-R_{2}^{a}\right) y(n) \mathbf{u}^{*}(n)\right\}
$$

\section{B.3 Função custo de Shalvi-Weinstein}

No caso da função custo de Shalvi-Weinstein da Equação (2.29, página 41), o gradiente é calculado como

$$
\nabla_{\mathbf{w}} J_{S W}(n)=\frac{\partial J_{S W}}{\partial \mathbf{w}^{*}}=\frac{\frac{\partial C_{2,2}^{y}}{\partial \mathbf{w}^{*}} \mathrm{E}\left\{|y(n)|^{2}\right\}^{2}-\frac{\partial \mathrm{E}\left\{|y(n)|^{2}\right\}^{2}}{\partial \mathbf{w}^{*}} C_{2,2}^{y}}{\mathrm{E}\left\{|y(n)|^{2}\right\}^{4}}
$$

Substituindo (B.11) e (B.12) em (B.15), após algumas manipulações algébricas simples chegase a

$$
\nabla_{\mathbf{w}} J_{S W}(n)=\frac{2(\beta-1)}{\mathrm{E}\left\{|y(n)|^{2}\right\}^{2}} \mathrm{E}\left\{\left(|y(n)|^{2}-R_{2}^{y}\right) y(n) \mathbf{u}^{*}\right\}
$$




\section{B.4 Demonstração da Equação (2.38)}

Calculando a hessiana da função custo de Godard reduzida a partir da Equação (2.37, página 42), tem-se

$$
\begin{gathered}
\frac{\partial \nabla_{\overline{\mathbf{w}}}^{H} \bar{J}_{G}}{\partial \overline{\mathbf{w}}^{*}}=2\left(\frac{R_{2}^{a}}{\beta+J_{S W}}\right)\left(-\frac{R_{2}^{a}}{\left(\beta+J_{S W}\right)^{2}} \frac{\partial J_{S W}}{\partial \overline{\mathbf{w}}^{*}}\right) \nabla_{\overline{\mathbf{w}}}^{H} J_{S W} \\
+\left(\frac{R_{2}^{a}}{\beta+J_{S W}}\right)^{2} \frac{\partial \nabla_{\overline{\mathbf{w}}}^{H} \bar{J}_{S W}}{\partial \overline{\mathbf{w}}^{*}}
\end{gathered}
$$

Essa equação pode ser reescrita como

$$
\mathbf{F}_{G}(\overline{\mathbf{w}})=\left(\frac{R_{2}^{a}}{\beta+J_{S W}}\right)^{2} \mathbf{F}_{S W}(\overline{\mathbf{w}})-2 \frac{\left(R_{2}^{a}\right)^{2}}{\left(\beta+J_{S W}\right)^{3}} \nabla_{\overline{\mathbf{w}}} J_{S W} \nabla_{\overline{\mathbf{w}}}^{H} J_{S W},
$$

o que leva à Equação (2.38). 


\section{Apêndice C}

\section{Sobre o algoritmo do Módulo}

\section{Constante Acelerado}

Neste apêndice, as principais considerações a respeito do algoritmo do Módulo Constante Acelerado, apresentadas no Capítulo 3, são justificadas. Inicialmente, o AC-CMA é deduzido. Em seguida, ele é interpretado como um algoritmo do tipo quase-Newton. Por fim, uma relação de conservação de energia é obtida.

\section{C.1 Dedução do AC-CMA}

Aplicando o método de Euler regressivo com passo de integração $\mu$ às equações (3.11a), (3.12) e (3.18), obtém-se

$$
\begin{aligned}
\mathbf{w}(n) & =\mathbf{w}(n-1)+\mu \mathbf{g}(n) \\
\mathbf{g}(n) & =\mathbf{g}(n-1)-\mu \mathbf{M}_{1}\left\{\mathbf{u}^{*}(n) \varepsilon(n)+2\left(\mathbf{M}_{2}+\mathbf{u}^{*}(n) \mathbf{u}^{T}(n) \mathbf{M}_{1} \mathbf{M}_{3}\right) \mathbf{g}(n)\right\} \\
\varepsilon(n) & =\varphi\left(\mathbf{u}^{T}(n) \mathbf{w}(n)\right) .
\end{aligned}
$$

A partir dessas equações não é possível atualizar $\mathbf{w}(n)$ e $\mathbf{g}(n)$. Esse problema pode ser resolvido, introduzindo-se um erro a priori, definido como

$$
e(n)=\varphi\left(\mathbf{u}^{T}(n) \mathbf{w}(n-1)\right)=\varphi(y(n))
$$

A função não-linear $\varphi(\cdot)$ pode ser escolhida a partir de uma "versão instantânea" da função custo de Godard [Godard, 1980]

$$
\Psi(n)=\frac{1}{2(\beta-1)}\left(|y(n)|^{2}-R_{2}^{a}\right)^{2},
$$


sendo $\beta=2(=3)$ para o caso complexo (real). Calculando o vetor gradiente dessa função em relação a w, obtém-se

$$
\nabla_{\mathbf{w}} \Psi(n)=e(n) \mathbf{u}^{*}(n)=\varphi(y(n)) \mathbf{u}^{*}(n)
$$

sendo

$$
\varphi(y(n))=e(n)=\left(|y(n)|^{2}-R_{2}^{a}\right) y(n)
$$

Multiplicando (C.1) por $\mathbf{u}^{T}(n)$ à esquerda, chega-se a

$$
\mathbf{u}^{T}(n) \mathbf{w}(n)=\mathbf{u}^{T}(n) \mathbf{w}(n-1)+\mu \mathbf{u}^{T}(n) \mathbf{g}(n) .
$$

Uma aproximação linear de $\varphi(\cdot)$ pode ser escrita como

$$
\begin{aligned}
\varphi\left(\mathbf{u}^{T} \mathbf{w}\right) & \approx \varphi\left(\mathbf{u}^{T} \mathbf{w}_{\mathbf{v}}\right)+\left[\left.\frac{\partial \varphi\left(\mathbf{u}^{T} \mathbf{w}\right)}{\partial \mathbf{w}}\right|_{\mathbf{w}=\mathbf{w}_{\mathbf{v}}}\right]^{T}\left(\mathbf{w}-\mathbf{w}_{\mathbf{v}}\right) \\
& \approx \varphi\left(\mathbf{u}^{T} \mathbf{w}_{\mathbf{v}}\right)+\breve{\varphi}\left(\mathbf{u}^{T} \mathbf{w}_{\mathbf{v}}\right) \mathbf{u}^{T}\left(\mathbf{w}-\mathbf{w}_{\mathbf{v}}\right)
\end{aligned}
$$

sendo

$$
\frac{\partial \varphi(y(n))}{\partial \mathbf{w}}=\breve{\varphi}(y(n)) \mathbf{u}(n)
$$

e

$$
\breve{\varphi}(y(n))=\left(\beta|y(n)|^{2}-R_{2}^{a}\right)
$$

Utilizando (C.8), (C.9), (C.7) e (C.11), o erro a posteriori, definido em (C.3), pode ser calculado a partir do erro a priori como

$$
\begin{aligned}
\varepsilon(n) & =\varphi\left(\mathbf{u}^{T}(n) \mathbf{w}(n-1)+\mu \mathbf{u}^{T}(n) \mathbf{g}(n)\right) \\
& \approx \varphi(y(n))+\mu \breve{\varphi}(y(n)) \mathbf{u}^{T}(n) \mathbf{g}(n) \\
& =e(n)+\mu\left(\beta|y(n)|^{2}-R_{2}^{a}\right) \mathbf{u}^{T}(n) \mathbf{g}(n) .
\end{aligned}
$$

Utilizando (C.12) e (C.2), obtém-se a seguinte expressão de atualização

$$
\mathbf{g}(n)=\mathbf{D}^{-1}(n)\left[\mathbf{g}(n-1)-\mu \mathbf{M}_{1} \mathbf{u}^{*}(n) e(n)\right]
$$

sendo

$$
\mathbf{D}(n)=\mathbf{I}+\mu^{2} \breve{\varphi}(y(n)) \mathbf{M}_{1} \mathbf{u}^{*}(n) \mathbf{u}^{T}(n)+2 \mu \mathbf{M}_{1}\left(\mathbf{M}_{2}+\mathbf{u}^{*}(n) \mathbf{u}^{T}(n) \mathbf{M}_{1} \mathbf{M}_{3}\right) .
$$


Utilizando o lema de inversão matricial [Marple, 1987; Haykin, 1996], pode ser mostrado que a matriz inversa $\mathbf{D}^{-1}(n)$ é dada por

$$
\mathbf{D}^{-1}(n)=\mathbf{A}\left\{\mathbf{I}-\frac{\mathbf{M}_{1} \mathbf{u}^{*}(n) \mathbf{u}^{T}(n) \mathbf{B}(n) \mathbf{M}_{1}^{-1}}{1+\mathbf{u}^{T}(n) \mathbf{B}(n) \mathbf{u}^{*}(n)}\right\}
$$

sendo

$$
\mathbf{A}=\left(\mathbf{I}+2 \mu \mathbf{M}_{1} \mathbf{M}_{2}\right)^{-1}
$$

e

$$
\mathbf{B}(n)=\mu\left\{\mu\left(\beta|y(n)|^{2}-R_{2}^{a}\right) \mathbf{I}+2 \mathbf{M}_{1} \mathbf{M}_{3}\right\} \mathbf{A M}_{1}
$$

Substituindo esse resultado na Equação (C.13), obtém-se uma primeira versão do algoritmo "acelerador" para equalização autodidata mostrada na Tabela C.1.

$$
\begin{aligned}
& \text { Inicialização do algoritmo: } \\
& R_{2}^{a}=\mathrm{E}\left\{|a(n)|^{4}\right\} / \mathrm{E}\left\{|a(n)|^{2}\right\} \\
& \mathbf{w}(0)=\left[\begin{array}{llllll}
0 & \cdots & 0 & 1 & 0 & \cdots
\end{array}\right]^{T}, \quad \mathbf{g}(0)=\mathbf{0} \\
& \mathbf{A}=\left(\mathbf{I}+2 \mu \mathbf{M}_{1} \mathbf{M}_{2}\right)^{-1} \\
& \beta=2(=3) \text { para o caso complexo (real) } \\
& \text { Para cada instante de tempo, } n=1,2, \cdots, \text { calcule: } \\
& y(n)=\mathbf{u}^{T}(n) \mathbf{w}(n-1) \\
& e(n)=\left(|y(n)|^{2}-R_{2}^{a}\right) y(n) \\
& \mathbf{B}(n)=\mu\left(\mu\left(\beta|y(n)|^{2}-R_{2}^{a}\right) \mathbf{I}+2 \mathbf{M}_{1} \mathbf{M}_{3}\right) \mathbf{A M}_{1} \\
& \mathbf{C}(n)=\frac{\mu e(n)+\mathbf{u}^{T}(n) \mathbf{B}(n) \mathbf{M}_{1}^{-1} \mathbf{g}(n-1)}{1+\mathbf{u}^{T}(n) \mathbf{B}(n) \mathbf{u}^{*}(n)} \mathbf{M}_{1} \\
& \mathbf{g}(n)=\mathbf{A}\left(\mathbf{g}(n-1)-\mathbf{C}(n) \mathbf{u}^{*}(n)\right) \\
& \mathbf{w}(n)=\mathbf{w}(n-1)+\mu \mathbf{g}(n)
\end{aligned}
$$

Tabela C.1: Sumário do AC-CMA matricial. 
Considerando as matrizes $\mathbf{M}_{k}=m_{k} \mathbf{I}, k=1,2,3$, com $m_{k}$ sendo constantes positivas, a complexidade computacional dessa versão se reduz de $M^{2}$ para $M$. Introduzindo as relações $m_{1} m_{2}=1 /(2 \kappa)$ e $m_{1} m_{3}=\kappa / 2$, chega-se à versão do AC-CMA da Tabela 3.3, página 80.

\section{C.2 O AC-CMA segundo o método quase-Newton}

Combinando (C.1) e (C.13), obtém-se

$$
\begin{aligned}
\mathbf{w}(n) & =\mathbf{w}(n-1)+\mu \mathbf{D}^{-1}(n)\left\{\mathbf{g}(n-1)-\mu \mathbf{M}_{1} \mathbf{u}^{*}(n) e(n)\right\}= \\
& =\mathbf{w}(n-1)-\mathbf{D}^{-1}(n)\left\{\mu^{2} \mathbf{M}_{1} \mathbf{u}^{*}(n) e(n)-\mathbf{w}(n-1)+\mathbf{w}(n-2)\right\} .
\end{aligned}
$$

Essa equação sugere que o AC-CMA pode ser interpretado como um algoritmo do tipo quaseNewton, revisitado na Seção 3.3. A versão matricial do AC-CMA (Tabela C.1) utiliza as seguintes estimativas para o vetor gradiente e matriz hessiana

$$
\widehat{\nabla}_{\mathbf{w}} J(n)=\beta_{\mathrm{AC}}\left\{\mu^{2} \mathbf{M}_{1} \mathbf{u}^{*}(n) e(n)-\mathbf{w}(n-1)+\mathbf{w}(n-2)\right\}
$$

e

$$
\widehat{\mathbf{F}}(n)=\beta_{\mathrm{AC}} \mathbf{D}(n),
$$

sendo $\beta_{\mathrm{AC}}$ uma constante positiva adequada e o passo de adaptação $\rho(n)=1$. Considerando a versão do AC-CMA da Tabela 3.3, utilizando (C.14) e assumindo $\beta_{\mathrm{AC}}=\kappa /(\mu+\kappa)$, obtêm-se

$$
\begin{aligned}
\rho(n) & =\mu^{2} m_{1} \beta_{\mathrm{AC}}=\frac{\mu^{2} m_{1} \kappa}{\mu+\kappa}, \\
\widehat{\nabla}_{\mathbf{w}} J(n) & =e(n) \mathbf{u}^{*}(n)-\frac{1}{\mu^{2} m_{1}}\{\mathbf{w}(n-1)-\mathbf{w}(n-2)\},
\end{aligned}
$$

$\mathrm{e}$

$$
\widehat{\mathbf{F}}(n)=\mathbf{I}+\rho(n)\left\{\breve{\varphi}(y(n))+\frac{\kappa}{\mu}\right\} \mathbf{u}^{*}(n) \mathbf{u}^{T}(n),
$$

sendo $\widehat{\nabla}_{\mathbf{w}} J(n)$ uma estimativa instantânea do gradiente $\mathrm{E}\left\{e(n) \mathbf{u}^{*}(n)\right\}$, "corrigida" por um termo proporcional ao incremento do vetor de coeficientes anterior e $\widehat{\mathbf{F}}(n)$ uma estimativa instantânea regularizada da matriz hessiana $\mathrm{E}\left\{\breve{\varphi}(y(n)) \mathbf{u}^{*}(n) \mathbf{u}^{T}(n)\right\}$. 


\section{C.3 O AC-CMA e a conservação de energia}

Na Seção 3.4, página 82, verificou-se que algoritmos da forma (3.24) obedecem a relação de conservação de energia de [Sayed, 2003, Cap. 6]. O AC-CMA não é um algoritmo da forma (3.24), já que a estimativa do gradiente é "corrigida" por um termo proporcional ao incremento do vetor de coeficientes, como mostrado na Equação (C.19).

Em contrapartida, a atualização dos coeficientes do AC-CMA da Tabela 3.3 é dada por

$$
\mathbf{w}(n)=\mathbf{w}(n-1)-\mu[-\mathbf{g}(n)]
$$

Desta equação verifica-se que o AC-CMA também pode ser interpretado como um algoritmo do gradiente estocástico sendo $-\mathbf{g}(n)$ uma estimativa do vetor gradiente. Da Tabela 3.3, o termo que atualiza $\mathbf{g}(n)$ é dado por $c(n) \mathbf{u}^{*}(n)$, ou seja

$$
\frac{b(n) \mathbf{u}^{T}(n) \mathbf{g}(n-1) \mathbf{u}^{*}(n)+\mu m_{1}(\mu+\kappa) \overbrace{e(n) \mathbf{u}^{*}(n)}^{(\mathrm{I})}}{\mu+\kappa+b(n)\|\mathbf{u}(n)\|^{2}} .
$$

Cabe notar que o termo $(\mathrm{I})=e(n) \mathbf{u}^{*}(n)=\widehat{\nabla}_{\mathbf{w}} J_{G}(n)$ é uma estimativa instantânea do vetor gradiente da função custo de Godard.

Para algoritmos do tipo gradiente estocástico, o operador de projeção oblíqua da Equação (3.34, página 84) se reduz ao operador de projeção ortogonal dado por

$$
\mathcal{V}(n)=\mathbf{I}-\frac{\mathbf{u}^{*}(n) \mathbf{u}^{T}(n)}{\|\mathbf{u}(n)\|^{2}}
$$

Subtraindo ambos os lados de (C.21) do vetor de coeficientes ótimos $\mathbf{w}_{\mathrm{o}}$, chega-se a

$$
\widetilde{\mathbf{w}}(n)=\widetilde{\mathbf{w}}(n-1)-\mu \mathbf{g}(n)
$$

Multiplicando essa equação e a recorrência de $\mathbf{g}(n)$ da Tabela 3.3 por $\mathcal{V}(n)$, obtêm-se

$$
\mathcal{V}(n) \widetilde{\mathbf{w}}(n)=\mathcal{V}(n) \widetilde{\mathbf{w}}(n-1)-\mu \mathcal{V}(n) \mathbf{g}(n)
$$

e

$$
\mathcal{V}(n) \mathbf{g}(n)=\frac{\kappa}{\mu+\kappa} \mathcal{V}(n) \mathbf{g}(n-1)
$$

Destas equações, verifica-se que o AC-CMA não satisfaz uma relação do tipo $\mathcal{V}(n) \widetilde{\mathbf{w}}(n)=$ $\mathcal{V}(n) \widetilde{\mathbf{w}}(n-1)$ e conseqüentemente não obedece a relação de conservação de energia considerando $\widetilde{\mathbf{w}}(n)$ ou $\mathbf{g}(n)$. 
Definindo uma combinação linear dos vetores $\widetilde{\mathbf{w}}(n)$ e $\mathbf{g}(n)$ como

$$
\widetilde{\mathbf{g}}(n) \triangleq \kappa \mathbf{g}(n)-\widetilde{\mathbf{w}}(n)
$$

e substituindo (C.24) nessa definição, obtém-se

$$
\widetilde{\mathbf{g}}(n)=(\mu+\kappa) \mathbf{g}(n)-\widetilde{\mathbf{w}}(n-1)
$$

A atualização de $\mathbf{g}(n)$ pode ser reescrita como forma

$$
\mathbf{g}(n)=\frac{\kappa}{\mu+\kappa} \boldsymbol{\Gamma}(n) \mathbf{g}(n-1)-\frac{\mu m_{1} \kappa e(n) \mathbf{u}^{*}(n)}{\mu+\kappa+b(n)\|\mathbf{u}(n)\|^{2}},
$$

sendo

$$
\boldsymbol{\Gamma}(n)=\mathbf{I}-\frac{b(n) \mathbf{u}^{*}(n) \mathbf{u}^{T}(n)}{\mu+\kappa+b(n)\|\mathbf{u}(n)\|^{2}} .
$$

Substituindo (C.29) em (C.28), obtém-se

$$
\widetilde{\mathbf{g}}(n)=\kappa \boldsymbol{\Gamma}(n) \mathbf{g}(n-1)-\widetilde{\mathbf{w}}(n-1)-\frac{\mu m_{1} \kappa(\mu+\kappa) e(n) \mathbf{u}^{*}(n)}{\mu+\kappa+b(n)\|\mathbf{u}(n)\|^{2}} .
$$

Seja $e_{a}(n)$ um erro a priori definido como $e_{a}(n) \triangleq \mathbf{u}^{T}(n) \widetilde{\mathbf{w}}(n-1)$. Somando e subtraindo o termo $b(n) \mathbf{u}^{*}(n) e_{a}(n) /\left(\mu+\kappa+b(n)\|\mathbf{u}(n)\|^{2}\right)$ no lado direito de (C.31), após algumas manipulações algébricas chega-se à seguinte equação recorrente para $\widetilde{\mathbf{g}}(n)$

$$
\widetilde{\mathbf{g}}(n)=\boldsymbol{\Gamma}(n) \widetilde{\mathbf{g}}(n-1)-\frac{\mu m_{1} \kappa(\mu+\kappa) e(n)+b(n) e_{a}(n)}{\mu+\kappa+b(n)\|\mathbf{u}(n)\|^{2}} \mathbf{u}^{*}(n) .
$$

Notando que $\mathcal{V}(n) \boldsymbol{\Gamma}(n)=\mathcal{V}(n)$ e $\mathcal{V}(n) \mathbf{u}^{*}(n)=\mathbf{0}$ e multiplicando (C.32) por $\mathcal{V}(n)$, obtém-se

$$
\mathcal{V}(n) \widetilde{\mathbf{g}}(n)=\mathcal{V}(n) \widetilde{\mathbf{g}}(n-1)
$$

Desta forma, o AC-CMA satisfaz uma relação o tipo $\mathcal{V}(n) \widetilde{\mathbf{w}}(n)=\mathcal{V}(n) \widetilde{\mathbf{w}}(n-1)$ para o vetor $\widetilde{\mathbf{g}}(n)$. Definindo erros a priori e a posteriori como

$$
\bar{\xi}_{a}(n)=\mathbf{u}^{T}(n) \widetilde{\mathbf{g}}(n-1)
$$

e

$$
\bar{\xi}_{p}(n)=\mathbf{u}^{T}(n) \widetilde{\mathbf{g}}(n),
$$

de (C.33), obtém-se a seguinte relação

$$
\widetilde{\mathbf{g}}(n)+\frac{\bar{\xi}_{a}(n) \mathbf{u}^{*}(n)}{\|\mathbf{u}(n)\|^{2}}=\widetilde{\mathbf{g}}(n-1)+\frac{\bar{\xi}_{p}(n) \mathbf{u}^{*}(n)}{\|\mathbf{u}(n)\|^{2}} .
$$


Calculando a norma ao quadrado de ambos os lados dessa relação, chega-se a

$$
\|\widetilde{\mathbf{g}}(n)\|^{2}+\bar{\mu}(n)\left|\bar{\xi}_{a}(n)\right|^{2}=\|\widetilde{\mathbf{g}}(n-1)\|^{2}+\bar{\mu}(n)\left|\bar{\xi}_{p}(n)\right|^{2}
$$

sendo

$$
\bar{\mu}(n)= \begin{cases}\frac{1}{\|\mathbf{u}(n)\|^{2}}, & \mathbf{u}(n) \neq \mathbf{0} \\ 0, & \text { caso contrário. }\end{cases}
$$

A relação (C.37) estabelece que o mapeamento das variáveis $\left\{\widetilde{\mathbf{g}}(n-1), \sqrt{\bar{\mu}(n)} \bar{\xi}_{p}(n)\right\}$ para as variáveis $\left\{\widetilde{\mathbf{g}}(n), \sqrt{\bar{\mu}(n)} \bar{\xi}_{a}(n)\right\}$ conserva a energia. Multiplicando (C.32) por $\mathbf{u}^{T}(n)$, obtém-se a seguinte relação entre os erros

$$
\bar{\xi}_{p}(n)=\frac{\mu+\kappa}{\mu+\kappa+b(n) / \bar{\mu}(n)} \bar{\xi}_{a}(n)-\frac{\mu m_{1} \kappa(\mu+\kappa) e(n)+b(n) e_{a}(n)}{(\mu+\kappa) \bar{\mu}(n)+b(n)}
$$

o que permite descrever o AC-CMA através de um mapeamento sem perdas com realimentação de ganho, como mostrado na Figura 3.4 da página 85. 


\section{Apêndice D}

\section{O algoritmo concorrente de Chen}

No contexto da adaptação autodidata do LTE, foi proposto em [De Castro et al., 2001] uma forma concorrente de operar o algoritmo de Decisão Direta (DD - Decision Direct) com o CMA para constelação M-QAM (M-Quadrature Amplitude Modulation). Com um aumento moderado na complexidade computacional, o CMA+DD apresenta uma considerável melhora de desempenho em relação ao CMA. Inspirando-se nessa idéia, Chen (2003) propôs uma modificação nesse esquema substituindo o algoritmo de Decisão Direta por um outro com Decisão Direta Suave (SDD - Soft Decision-Direct). O CMA+SDD, também chamado de bootstrap maximum a posteriori probability [Chen et al., 2004a], possui uma complexidade computacional menor e uma taxa de convergência mais rápida do que o CMA+DD. Além disso, esses algoritmos concorrentes têm capacidade de corrigir rotações de fase em constelação $M$-QAM não-múltiplas de $\pi / 2$.

No esquema concorrente proposto por De Castro et al. (2001), a atualização do algoritmo de Decisão Direta é feita depois da atualização do CMA. Seus coeficientes são ajustados apenas se a adaptação do CMA tiver sido considerada correta com uma alta probabilidade. Isto é feito para evitar propagação de erros devido às decisões incorretas [De Castro et al., 2001; Chen, 2003]. No esquema concorrente de Chen (2003; 2004), utiliza-se a SDD ao invés da Decisão Direta e a adaptação dos coeficientes do CMA e do algoritmo SDD ocorre simultaneamente. Esse algoritmo é revisitado a seguir.

Assume-se que o vetor de coeficientes do LTE é dado por

$$
\mathbf{w}(n)=\mathbf{w}_{c}(n)+\mathbf{w}_{d}(n),
$$


sendo $\mathbf{w}_{c}$ e $\mathbf{w}_{d}$ os vetores de coeficientes adaptados respectivamente pelo CMA e pelo algoritmo SDD e que a saída do equalizador é $y(n)=\mathbf{u}^{T}(n) \mathbf{w}(n-1)$.

Após uma convergência "satisfatória", a saída do equalizador pode ser expressa aproximadamente como

$$
y(n) \approx a\left(n-\tau_{d}\right)+v(n)
$$

sendo $v(n)=v_{R}(n)+j v_{I}(n)$ um ruído do tipo AWGN com $\mathrm{E}\left\{v_{R}(n) v_{I}(n)\right\}=0$ e

$$
\rho \triangleq \mathrm{E}\left\{v_{R}^{2}(n)\right\}=\mathrm{E}\left\{v_{I}^{2}(n)\right\}
$$

Desta forma, a função densidade de probabilidade (f.d.p.) a posteriori de $y(n)$ pode ser aproximada por

$$
p(y(n)) \approx \sum_{k=1}^{K} \sum_{l=1}^{K} \frac{P_{k l}}{2 \pi \rho} \exp \left[-\frac{\left|y(n)-a_{k l}\right|^{2}}{2 \rho}\right],
$$

sendo $a_{k l}$ símbolos de uma constelação $M$-QAM que pertencem ao conjunto

$$
A=\left\{a_{k l}=(2 k-K-1)+j(2 l-K-1), 1 \leq k, l \leq K\right\}
$$

com $K=\sqrt{M}$ e $P_{k l}$ as probabilidades a priori de $a_{k l}$. Para símbolos equiprováveis $P_{k l}=$ $1 / M, \quad 1 \leq \operatorname{com} k, l \leq K$.

O plano complexo pode ser dividido em $M / 4$ regiões regulares. Neste caso, como indicado na Figura D.1, cada região $A_{i}$ contém quatro símbolos, isto é,

$$
A_{i}=\left\{a_{i m}, m=1,2,3,4\right\}
$$

sendo $i=1,2, \ldots, M / 4$. Definindo

$$
\varepsilon_{i m}(n)=y(n)-a_{i m},
$$

se a saída do equalizador estiver na região $A_{i}$, uma aproximação local para a f.d.p a posteriori de $y(n)$ será

$$
\hat{p}_{i}(y(n)) \approx \sum_{m=1}^{4} \frac{1}{8 \pi \rho} \exp \left[-\frac{\left|\varepsilon_{i m}(n)\right|^{2}}{2 \rho}\right] .
$$

Cabe observar que neste caso a probabilidade a priori é igual a $1 / 4$.

O algoritmo SDD minimiza o critério definido pelo logaritmo da f.d.p local de $y(n)$, ou seja,

$$
J_{M A P}=-\mathrm{E}\left\{\rho \log \left[\hat{p}_{i}(y(n))\right]\right\}
$$


Utilizando um algoritmo do gradiente estocástico para ajustar $\mathbf{w}_{d}(n)$, chega-se a

$$
\mathbf{w}_{d}(n)=\mathbf{w}_{d}(n-1)-\mu_{d} \xi(n) \mathbf{u}^{*}(n)
$$

sendo

$$
\xi(n)=\frac{\sum_{m=1}^{4} \exp \left[-\frac{\left|\varepsilon_{i m}(n)\right|^{2}}{2 \tilde{\rho}}\right] \varepsilon_{i m}(n)}{\sum_{m=1}^{4} \exp \left[-\frac{\left|\varepsilon_{i m}(n)\right|^{2}}{2 \tilde{\rho}}\right]} .
$$

Para assegurar uma correta separação dos agrupamentos, o parâmetro $\tilde{\rho}$ deve ser menor que a metade da mínima distância entre dois símbolos vizinhos da constelação [Chen et al., 1995]. Essa é uma "estimativa" do valor de $\rho$ definido em (D.3). Para se obter uma melhor convergência, a escolha desse parâmetro deve ser feita em cada etapa, o que pode ser tornar uma tarefa difícil [Chen, 2003].

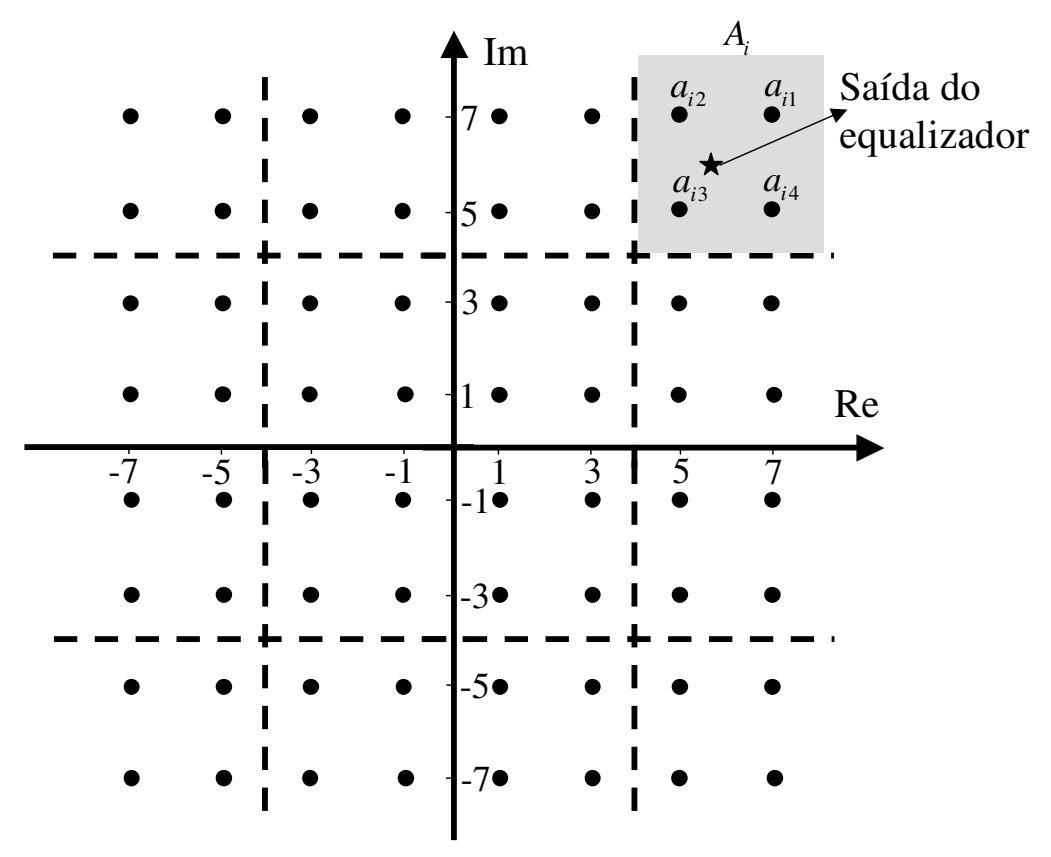

Figura D.1: Ilustração das regiões locais para adaptação SDD e 64-QAM.

Como indicado em (D.9), na adaptação dos coeficientes do algoritmo SDD é necessário calcular apenas $4 \exp (\cdot)$ em cada iteração, o que pode ser implementado através de uma tabela. A tarefa a ser executada para uma constelação $M$-QAM é atingida com $L$ etapas, sendo $L=\log _{2} \sqrt{M}$. A identificação da região $A_{i}$ não causa um aumento significativo na complexidade computacional já que isso requer apenas $2(L-1)$ comparações. Desta forma, a complexidade do algoritmo é sempre equivalente à complexidade mínima do caso 4-QAM 
[Chen et al., 1995]. Considerando por exemplo 64-QAM, o plano complexo é dividido em $64 / 4=16$ regiões com 4 símbolos como mostrado na Figura D.1. A primeira etapa consiste na classificação da saída do equalizador em um dos 4 quadrantes do plano complexo. Supondo que uma amostra da saída é $5,3+j 5,7$, ela deve ser classificada no primeiro quadrante, dado que sua parte real e sua parte imaginária são positivas. Na segunda etapa a saída deve ser classificada nas 4 regiões existentes no primeiro quadrante. Por fim, na última etapa, $\xi(n)$ pode ser calculado através da Equação (D.9), utilizando-se os 4 símbolos $a_{i m}, m=1,2,3,4$ da região final $A_{i}$.

As operações do concorrente CMA+SDD estão mostradas na Tabela D.1. Ele possui uma complexidade computational que fica entre as complexidades do CMA e do concorrente CMA+DD [Chen, 2003].

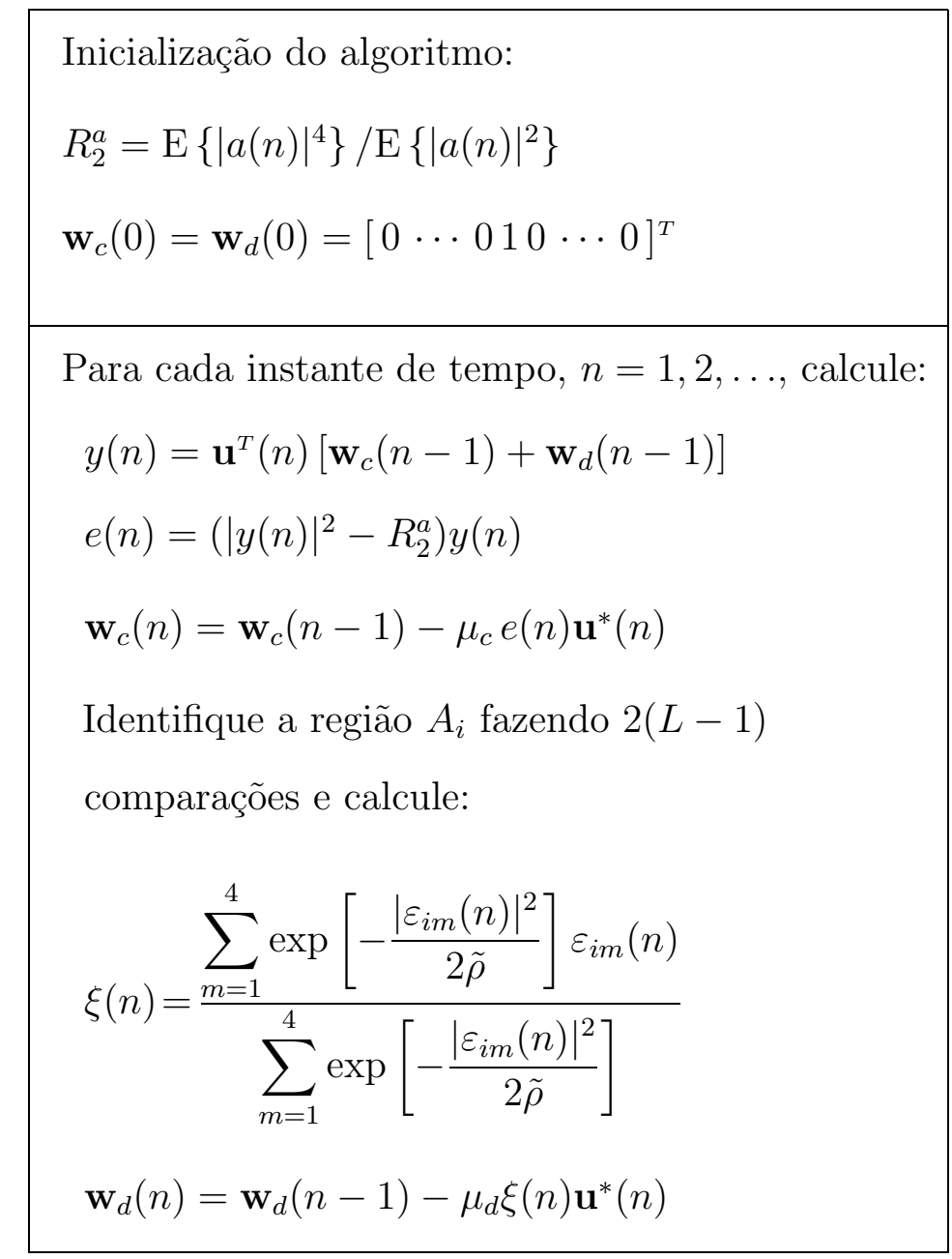

Tabela D.1: Sumário do CMA+SDD. 


\section{Apêndice E}

\section{O algoritmo RTRL autodidata}

Como mostrado na Seção 5.4, a dinâmica da RNN, esquematizada na Figura 5.2 (página 138), é descrita pelas seguintes equações

$$
\begin{aligned}
& v_{k}(n+1)=\sum_{l=1}^{N} w_{k l}(n) y_{l}(n)+\sum_{l=0}^{M-1} w_{k l^{\prime}}(n) x_{l}(n), \\
& y_{k}(n+1)= \begin{cases}v_{k}(n+1), & k=s \\
\chi\left(v_{k}(n+1)\right), & k \neq s,\end{cases}
\end{aligned}
$$

sendo $l^{\prime}=l+N+1, y_{k}(n)$ a saída do $k$-ésimo neurônio, $s$ o neurônio de saída, $\chi\left(v_{k}\right)=$ $\varphi\left(v_{k_{R}}\right)+j \varphi\left(v_{k_{I}}\right)$ a função de ativação com $v_{k_{R}}$ e $v_{k_{I}}$ as partes real e imaginária de $v_{k}$ e $\varphi(\cdot)$ a função sigmoidal [Kechriotis et al., 1994a; Haykin, 1999].

Para obter o algoritmo RTRL introduzem-se inicialmente os termos de sensibilidade $P_{R R}$, $P_{R I}, P_{I R}$ e $P_{I I}$, definidos como [Kechriotis et al., 1994a; Kechriotis \& Manolakos, 1994b]

$$
\left[\begin{array}{cc}
P_{R R p q}^{k} & P_{R I p q}^{k} \\
P_{I R p q}^{k} & P_{I I p q}^{k}
\end{array}\right]_{n} \triangleq\left[\begin{array}{cc}
\frac{\partial y_{k_{R}}}{\partial w_{p q_{R}}} & \frac{\partial y_{k_{R}}}{\partial w_{p q_{I}}} \\
\frac{\partial y_{k_{I}}}{\partial w_{p q_{R}}} & \frac{\partial y_{k_{I}}}{\partial w_{p q_{I}}}
\end{array}\right]_{n},
$$

sendo que o subscrito $n$ indica a dependência das variáveis com o índice de tempo. Considerando a função custo de Godard [Godard, 1980]

$$
J_{G}=\mathrm{E}\left\{\left(\left|y_{s}(n)\right|^{2}-R_{2}^{a}\right)^{2}\right\}
$$

o gradiente dessa função em relação a $w_{p q}$ é dado por (ver Apêndice B.1)

$$
\nabla_{w_{p q}} J_{G}=\frac{\partial J_{G}}{\partial w_{p q_{R}}}+j \frac{\partial J_{G}}{\partial w_{p q_{I}}} .
$$


Derivando $J_{G}$ em relação a $w_{p q_{R}}$ e $w_{p q_{I}}$ e tomando o valor instantâneo, obtêm-se

$$
\frac{\partial \widehat{J}_{G}}{\partial w_{p q_{R}}}=4\left(\left|y_{s}(n)\right|^{2}-R_{2}^{a}\right)\left[y_{s_{R}}(n) \frac{\partial y_{s_{R}}(n)}{\partial w_{p q_{R}}}+y_{s_{I}}(n) \frac{\partial y_{s_{I}}(n)}{\partial w_{p q_{R}}}\right]
$$

e

$$
\frac{\partial \widehat{J}_{G}}{\partial w_{p q_{I}}}=4\left(\left|y_{s}(n)\right|^{2}-R_{2}^{a}\right)\left[y_{s_{R}}(n) \frac{\partial y_{s_{R}}(n)}{\partial w_{p q_{I}}}+y_{s_{I}}(n) \frac{\partial y_{s_{I}}(n)}{\partial w_{p q_{I}}}\right]
$$

Deve-se então encontrar uma equação recursiva para o cálculo dos termos $P_{R R}, P_{R I}, P_{I R}$ e $P_{I I}$. Considerando a parte real de (E.2) e derivando em relação à $w_{p q_{R}}(n)$, obtém-se

$$
\frac{\partial y_{k_{R}}(n+1)}{\partial w_{p q_{R}}(n)}=f_{R}^{k}(n+1) \frac{\partial v_{k_{R}}(n+1)}{\partial w_{p q_{R}}(n)}
$$

sendo

$$
f_{R}^{k}(n+1)=\left\{\begin{array}{cc}
1, & k=s \\
\varphi^{\prime}\left(v_{k_{R}}(n+1)\right), & k \neq s
\end{array} .\right.
$$

A parte real de (E.1) pode ser escrita como

$$
v_{k_{R}}(n+1)=\sum_{l=1}^{N}\left[w_{k l_{R}} y_{l_{R}}(n)-w_{k l_{I}} y_{l_{I}}(n)\right]+\sum_{l=0}^{M-1}\left[w_{k l^{\prime} R} x_{l_{R}}(n)-w_{k l_{I}} x_{l_{I}}(n)\right] .
$$

Utilizando (E.3) e derivando (E.10) em relação a $w_{p q_{R}}$, chega-se a

$$
P_{R R p q}^{k}(n+1)=f_{R}^{k}(n+1)\left[\sum_{m=1}^{N}\left[w_{k m R} P_{R R p q}^{m}(n)-w_{k m I} P_{I R p q}^{m}(n)\right]+\delta_{p k} x_{q R}(n)\right],
$$

sendo

$$
\delta_{p k}=\left\{\begin{array}{cc}
1, & p=k \\
0, & p \neq k
\end{array}\right.
$$

De forma similar, obtêm-se equações recursivas para $P_{R I}, P_{I R}$ e $P_{I I}$, que podem ser agrupadas na seguinte equação matricial

$$
\begin{aligned}
{\left[\begin{array}{cc}
P_{R R p q}^{k} & P_{R I p q}^{k} \\
P_{I R p q}^{k} & P_{I I p q}^{k}
\end{array}\right]_{n+1}=\left[\begin{array}{cc}
f_{R}^{k} & 0 \\
0 & f_{I}^{k}
\end{array}\right]_{n+1} \times } \\
\left(\sum_{l=1}^{N}\left[\begin{array}{ll}
w_{k l_{R}} & -w_{k l_{I}} \\
w_{k l_{I}} & w_{k l_{R}}
\end{array}\right]_{n}\left[\begin{array}{cc}
P_{R R p q}^{l} & P_{R I p q}^{l} \\
P_{I R p q}^{l} & P_{I I p q}^{l}
\end{array}\right]_{n}+\delta_{p k}\left[\begin{array}{cc}
x_{q_{R}} & -x_{q_{I}} \\
x_{q_{I}} & x_{q_{R}}
\end{array}\right]_{n}\right) .
\end{aligned}
$$


Esses parâmetros de sensibilidade devem ser inicializados com zero [Kechriotis \& Manolakos, 1994b]. Finalmente, a atualização dos pesos deve ser feita utilizando-se a seguinte equação

$$
\begin{aligned}
w_{p q}(n+1) & =w_{p q}(n)-\mu \widehat{\nabla}_{w_{p q}} J_{G}= \\
& =w_{p q}(n)-\mu \sum_{k=1}^{N}\left[e_{k_{R}} e_{k_{I}}\right]_{n}\left[\begin{array}{cc}
P_{R R p q}^{k} & P_{R I p q}^{k} \\
P_{I R p q}^{k} & P_{I I p q}^{k}
\end{array}\right]_{n}\left[\begin{array}{l}
1 \\
j
\end{array}\right]
\end{aligned}
$$

sendo

$$
e_{k}(n)= \begin{cases}\left(\left|y_{k}(n)\right|^{2}-R_{2}^{a}\right) y_{k}(n), & k=s \\ 0, & k \neq s\end{cases}
$$

